\title{
Optical excitations in electron microscopy
}

\author{
F. J. García de Abajo* \\ Instituto de Óptica-CSIC, Serrano 121, 28006 Madrid, Spain \\ (Published 3 February 2010)
}

\begin{abstract}
This review discusses how low-energy valence excitations created by swift electrons can render information on the optical response of structured materials with unmatched spatial resolution. Electron microscopes are capable of focusing electron beams on subnanometer spots and probing the target response either by analyzing electron energy losses or by detecting emitted radiation. Theoretical frameworks suited to calculate the probability of energy loss and light emission (cathodoluminescence) are reconsidered and compared with experimental results. More precisely, a quantum-mechanical description of the interaction between the electrons and the sample is discussed, followed by a powerful classical dielectric approach that can be applied in practice to more complex systems. The conditions are assessed under which classical and quantum-mechanical formulations are equivalent. The excitation of collective modes such as plasmons is studied in bulk materials, planar surfaces, and nanoparticles. Light emission induced by the electrons is shown to constitute an excellent probe of plasmons, combining subnanometer resolution in the position of the electron beam with nanometer resolution in the emitted wavelength. Both electron energy-loss and cathodoluminescence spectroscopies performed in a scanning mode of operation yield snapshots of plasmon modes in nanostructures with fine spatial detail as compared to other existing imaging techniques, thus providing an ideal tool for nanophotonics studies.
\end{abstract}

DOI: 10.1103/RevModPhys.82.209 PACS number(s): 68.37.Lp, 79.20.Uv, 78.60.Hk, 73.20.Mf

\section{CONTENTS}

I. Introduction

A. Spectroscopy using electron microscopes

II. Interaction of Swift Electrons with Matter

A. An evanescent source of light in matter

B. Classical dielectric formalism

1. Nonretarded approximation

2. Retardation effects

C. Quantum approach

1. Quantum description of the target

2. Quantum effects in the fast electrons

III. Electron Energy-Loss Spectroscopy

A. Space, momentum, and energy resolution

B. Bulk losses and determination of bulk dielectric functions

C. Planar surfaces

1. Excitation of surface plasmons and surface plasmon polaritons

2. Guided modes in thin films

D. Curved geometries

1. Cylinders

2. Spheres

3. Coupled nanoparticles

E. More complex shapes

1. Analytical methods
a. Ellipsoids
b. Wedges
c. Supported particles

2. Nonretarded boundary element method

*jga@cfmac.csic.es
3. Retarded boundary element method

4. Multiple-scattering approach

F. Composite materials

G. Carbon molecules and low-dimensional structures: The discrete-dipole approximation

H. Relation to the photonic local density of states

I. Electronic structure determination

IV. Cathodoluminescence: Generation of Light by Incoming Electrons

A. Mechanisms of light emission

1. Coherent electron-induced radiation emission

2. Incoherent cathodoluminescence

B. Calculation of coherent light emission

C. Transition radiation

D. Cherenkov radiation

1. Cherenkov effect in photonic crystals

E. Diffraction radiation

1. Smith-Purcell emission

F. Cathodoluminescence and plasmons

1. Plasmons in metallic films and gratings

2. Plasmon mapping

G. Ultrafast cathodoluminescence

V. Related Phenomena and Suggested Experiments

A. Mechanical momentum transfer

B. Vicinage effects

1. Interaction between two electrons

2. Electron self-interaction

C. Electron energy-gain spectroscopy

D. Surface plasmon launching

E. Nonlocal effects in nanostructured metals

VI. Prospects for Plasmonics

VII. Conclusion

Acknowledgments 
Appendix A: Dielectric Response of Homogeneous Media

1. Lindhard and Mermin dielectric functions

256

2. Hydrodynamic model

Appendix B: Nonretarded Screened Interaction in Simple Geometries

1. Planar surface

2. Cylinder

3. Sphere

Appendix C: Retarded Scattering and Coupling Coefficients in a Sphere Including Nonlocal Effects

Nomenclature

List of selected symbols

List of selected acronyms

References

\section{INTRODUCTION}

Investigation of minute physical processes has been essential for advancing knowledge and generating the right questions all the way from the beginning of modern science up to recent developments in nanotechnology. Far-field optical microscopes have contributed to this end, but they are limited by diffraction to a spatial resolution of about half the light wavelength in practice. Actually, the best resolution $(<1 \AA)$ is currently achieved using electrons rather than light (Ruska, 1987; Nellist et al., 2004); for electrons the de Broglie wavelength (de Broglie, 1925) is well below $0.1 \AA$ at energies above $20 \mathrm{keV}$.

Besides acquiring static images of the nanoworld, we are interested in finding out about the excitations that small objects can sustain, which inform us about their dynamical evolution and are relevant for encoding and manipulating information and for exploring a myriad of applications in fields such as molecular biology. Thus, our ultimate goal is to perform spectroscopy at the shortest possible length scale with the highest energy resolution. With this focus in mind, we can classify the available experimental techniques as shown in Fig. 1, which clearly indicates that electron-based spectroscopies offer the best choice for optimizing spatial resolution.

Two routes have been devised so far for dealing with the diffraction limit: ${ }^{1}$ (1) reduction of the wavelength of the probe and (2) employment of near-field detection techniques. (1) Moving to shorter photon wavelengths is difficult due to the lack of versatile lenses and mirrors beyond the ultraviolet. Nonetheless, recent advances in $\mathrm{x}$-ray microscopy (XRM) have allowed $15 \mathrm{~nm}$ imaging resolution (Chao et al., 2005) at energies above $250 \mathrm{eV}$. Alternatively, shifting from photons to electrons of the same energy encompasses a significant decrease in wave-

\footnotetext{
${ }^{1}$ The existence of a diffraction limit has been brought into question by recent investigations of superoscillating functions (Zheludev, 2008).
}

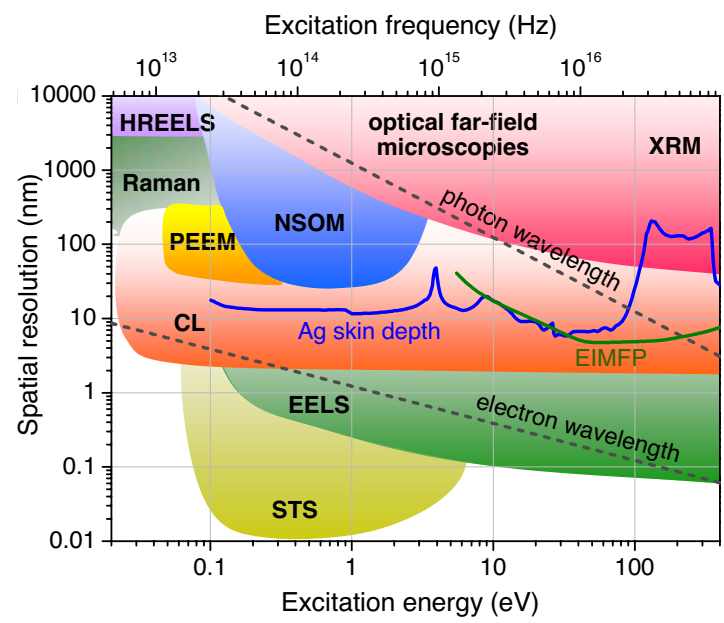

FIG. 1. (Color online) Atlas of spatially resolved spectroscopy techniques. They are organized according to their space and energy resolution (see Nomenclature for a list of acronyms). The relation between wavelength and energy is represented by dashed curves for photons and electrons. The universal electron inelastic mean free path (EIMFP) is given as a function of electron energy. The skin depth of Ag is calculated from optical data compiled by Palik, 1985 .

length and improved spatial resolution. ${ }^{2}$ (2) Exploitation of the near field is another option, particularly when it relies on evanescent components, the fast decay of which provides an extra handle for enhancing resolution using localized probes. For instance, near-field scanning optical microscopy (NSOM) (based on a subwavelength tip at the end of a fiber that brings, collects, or scatters light) and tip-enhanced NSOM can push spatial resolution down to tens of nanometers (Betzig et al., 1992; Hartschuh, 2008). Even better detail in the subangstrom domain is routinely achieved using the previously developed scanning tunneling microscope (STM), on which scanning tunneling spectroscopy (STS) yields $0.1 \mathrm{eV}$ energy resolution (Hörmandinger, 1994). However, STS works only with metals, and its extreme spatial accuracy $(\sim 0.01 \AA$ in the vertical direction) requires close proximity between the tip and the sample surface, so that single-electron excitations dominate the spectra and mask collective modes relevant to optics, such as plasmons.

Electron microscopes are thus the best option for resolving both localized and extended excitations with subnanometer spatial detail and $<0.1 \mathrm{eV}$ energy resolution in any type of material (Lazar et al., 2006). These instruments are sufficiently versatile to be surface sensitive and to simultaneously procure information on bulk properties. Their performance has considerably improved in recent years due to extraordinary advances in

\footnotetext{
${ }^{2}$ Heavier particles such as protons have been argued to provide good spatial resolution compared to electrons (Demkov and Meyer, 2004). Actually, a helium ion microscope has recently been released (Ward et al., 2006) and achieved $2.4 \AA$ resolution (product released by Carl Zeiss in 2008).
} 


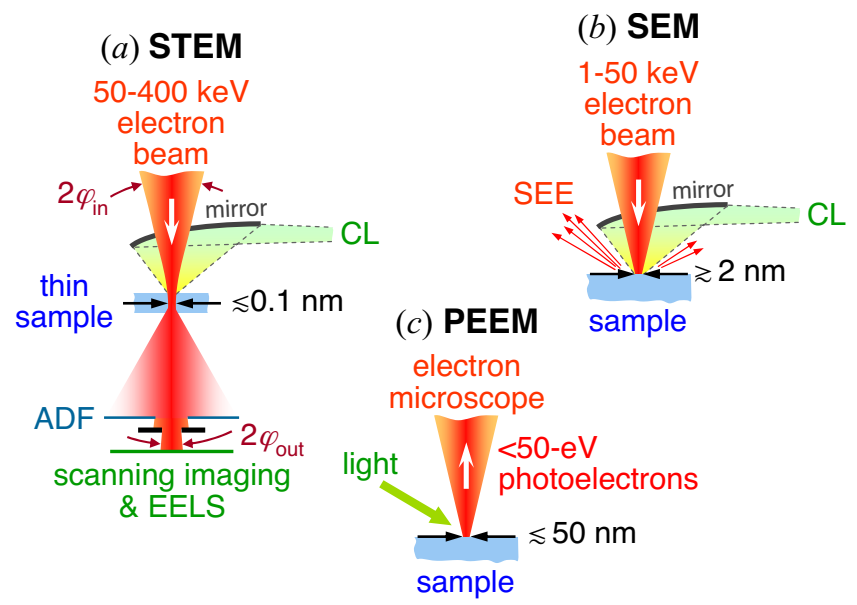

FIG. 2. (Color online) Schematic representation of various types of electron microscope equipped to perform spectroscopy. (a) Scanning transmission electron microscopes (STEMs) allow analysis of the energy distribution of electrons transmitted through a thin specimen ( $\$ 100 \mathrm{~nm}$ thick), on which the incident beam is focused down to a $<0.1 \mathrm{~nm}$ spot. Largemomentum transfers are collected through annular dark-field detectors (ADFs). Cathodoluminescence (CL) light detection is also possible on a STEM. (b) Scanning electron microscopes (SEMs) collect secondary electrons (SEs) to form images or CL emission to perform spectroscopy, with spatial resolution down to $\sim 2 \mathrm{~nm}$. (c) Photoemission electron microscopes (PEEMs) can render spectroscopic information on the photoexcitation process and electron-hole $(e-h)$ dynamics with $\$ 50 \mathrm{~nm}$ resolution.

energetic electron sources and optics. A number of studies in different fields benefit from the unprecedented spatial resolution of electron microscopy, which no other technique can currently match.

Electron microscopy has different flavors depending on the type of signal that we measure [inelastic losses, cathodoluminescence (CL) light emission, secondary electrons (SEs), etc.]. Several widely available types of setup are schematically shown in Fig. 2. The transmission electron microscope (TEM) provides by far the most powerful combination of spectral and energy resolution, mainly via analysis of loss events. This type of microscope can operate like a conventional optical microscope, projecting a bright image of an object on a phosphor screen or a charge-coupled device camera to picture the magnified sample. Energy-filtered TEM (EFTEM) allows construction of images out of those electrons that have donated a determined amount of energy to the specimen, which is useful to visualize selected losses [for instance, core excitations identifying the chemical environment of atomic species (Batson, 1993)]. Excellent spatial resolution is obtained using TEMs operated in scanning mode (STEM), in which the beam is focused and scanned over the sampled area, either to form images out of large-momentum transfers recorded by annular dark-field electron detectors or to collect loss spectra at specific locations.

Most TEMs use energetic electrons in the range of $50-400 \mathrm{keV}$ and require very thin specimens
( $\lesssim 100 \mathrm{~nm}$ ) that are relatively transparent to these electrons. Sample preparation is thus an important issue for achieving optimum spatial resolution.

In contrast, scanning electron microscopes (SEMs) can work with any sample that is covered by a thin metal layer $(\sim 1-2 \mathrm{~nm})$, forming images by collecting SEs upon bombardment with a primary beam of $\gtrsim 2 \mathrm{~nm}$ in diameter. Furthermore, one can perform spectroscopy on SEMs through wavelength-resolved CL emission. Although CL spectroscopy can also be collected on a TEM (Yamamoto, Sugiyama, and Toda, 1996), it is most commonly available in SEMs equipped with a light collector system (e.g., an ellipsoidal mirror) and a photon spectrometer.

Low-energy electron microscopy (LEEM) (Rocca, 1995) relies on the use of $1-100 \mathrm{eV}$ electrons and can achieve $<50 \mathrm{~nm}$ spatial resolution accompanied by subelectronvolt energy resolution. As an example of application, LEEM has been recently used to observe acoustic plasmons (Diaconescu et al., 2007). In contrast, photoemission electron microscopy (PEEM) (Bauer, 1994) is particularly useful to map the light intensity in illuminated nanostructures, as recently demonstrated for lithographically patterned Ag nanoparticle arrays (Cinchetti et al., 2005; Aeschlimann et al., 2007). The femtosecond dynamics of plasmon excitations has also been resolved using multiphoton PEEM with pumpprobe illumination (Kubo et al., 2005, 2007).

Finally, there are other configurations that make use of electrons to perform spectroscopy. For instance, reflection electron energy-loss spectroscopy (REELS) has recently been used to determine optical properties of noble metals (Werner, 2006; Werner et al., 2007; Went et al., 2008) and graphite (Calliari et al., 2008) after careful data analysis.

\section{A. Spectroscopy using electron microscopes}

A swift electron impinging on a microscope specimen gives rise to secondary electron emission (SEE) and CL that can be energy analyzed, thus yielding information on the excitation spectrum of the sample. Electron energy-loss spectroscopy (EELS) performed in STEMs adds up to this suite of probes. The main advantage of electron microscopes is that these types of spectroscopies can be performed with truly nanometer spatial resolution.

Unlike freely propagating light, the bare field of an electron is evanescent, as shown in Sec. II.A. This is advantageous in investigating localized excitations, involving wave-vector components of the electromagnetic field that lie outside the light cone. For example, electron beams were instrumental in discovering and characterizing collective excitations of conduction electrons in metals (Ruthermann, 1948; Watanabe, 1956; Ritchie, 1957; Powell and Swan, 1959; Chen and Silcox, 1975b; Raether, 1980), known as plasmons because they are quasiparticles associated with oscillations of the electron gas plasma. Specifically, bulk plasmons in a homogeneous metal are conspicuous in EELS since they are 


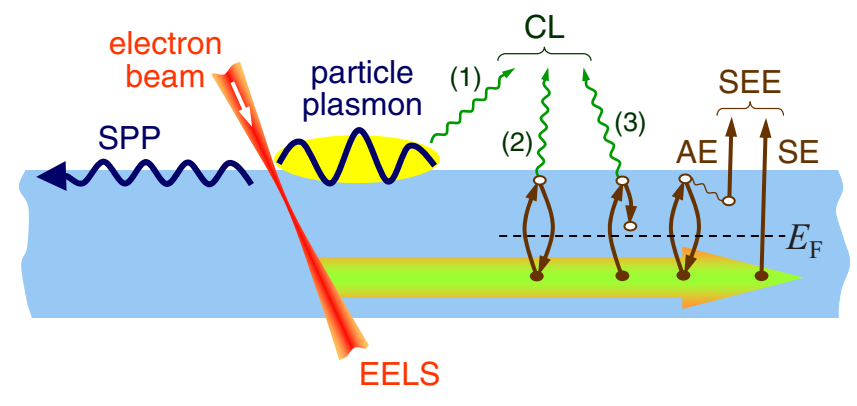

FIG. 3. (Color online) Schematic representation of some excitation processes triggered in a solid by a swift passing electron. We emphasize their connection to measured signals in electron microscopes: CL, secondary electron emission (SEE), and electron energy-loss spectroscopy (EELS). The electron can launch propagating surface plasmon polaritons (SPPs; see Sec. V.D). It can also produce surface plasmons localized in nanoparticles or in surface features (Sec. VI). Localized surface plasmons typically decay by coupling to radiation, thus giving rise to contribution (1) to CL (Sec. IV.A.1). Electronic excitations in the sample (e.g., $e$ - $h$ pairs) can decay radiatively back to the initial state [coherent CL emission (2), automatically included in the random-phase approximation (RPA) dielectric function as a bubble diagram (Lindhard, 1954); see Sec. IV.A.1] or to a different excited state (incoherent CL emission; Sec. IV.A.2). In metals, secondary electronic excitations constitute the dominant decay channel, producing Auger electrons (AEs), sometimes above the vacuum level, so that they contribute to the detected SEE. Direct excitation of electrons from the target is also possible, producing true SEs.

electrostatic and longitudinal in nature, ${ }^{3}$ and for this reason they couple efficiently to moving charges.

Similarly, plasmons can be confined at the surface of a metal, and they are actually the source of interesting phenomena and applications that comprise the field of plasmonics (see Sec. VI). Surface plasmons (SPs) are versatile entities: they can be trapped in metal particles that are much smaller than the wavelength (Myroshnychenko, Rodríguez-Fernández, et al., 2008), and they can hybridize with light extending over larger metallic structures (Coyle et al., 2001). More precisely, surface plasmon polaritons (SPPs), which are a subset of SPs capable of propagating on planar surfaces (Barnes et al., 2003; Ozbay, 2006; Zia et al., 2006) or along onedimensional (1D) waveguides (Bozhevolnyi et al., 2006), hold great promise of becoming the natural link between current nanoelectronics and future integrated nanophotonics, operating at frequencies that are $\sim 10^{5}$ times higher than microchip clocks.

As shown in Fig. 3, the passage of a fast electron

\footnotetext{
${ }^{3}$ Bulk plasmons in a source-free metal are characterized by zero magnetic field and longitudinal electric field $(\nabla \times \mathbf{E}=0)$, so that they trivially satisfy Maxwell's equations under the condition of vanishing permittivity. In contrast, surface plasmons are confined to metal-dielectric interfaces, they involve nonzero magnetic fields, and they have transverse character $(\nabla \cdot \mathbf{E}=0$ in each homogeneous region of space separated by the interface on which plasmons are defined).
}

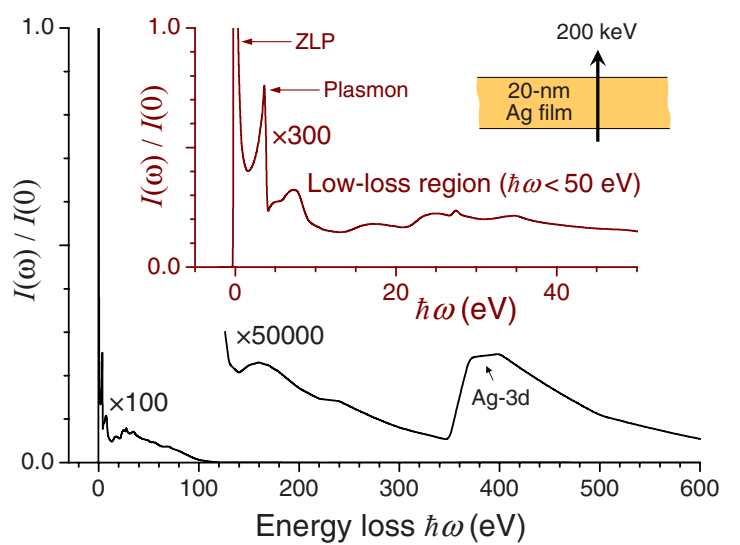

FIG. 4. (Color online) Typical electron energy-loss spectrum. We can identify a narrow zero-loss peak (ZLP; FWHM $\approx 0.2 \mathrm{eV}$ ), collective modes in the valence loss region (e.g., the $3.7 \mathrm{eV}$ plasmon of $\mathrm{Ag}$ in the inset), and much weaker core excitations at higher lost energies. The spectrum has been calculated for $200 \mathrm{keV}$ electrons traversing a $20 \mathrm{~nm} \mathrm{Ag}$ film using optical data for Ag (Palik, 1985) and assuming a collection angle of $5 \mathrm{mrad}$.

can excite localized plasmons in metallic nanoparticles, but it can also launch SPPs in planar metallic surfaces (Heitmann, 1977; Bashevoy et al., 2006; van Wijngaarden et al., 2006) or in metal nanowires (Vesseur et al., 2007). Quite different from SPPs, localized plasmons can decay radiatively, thus contributing to $\mathrm{CL}$, although all sorts of plasmons can partially decay via inelastic channels that involve electronic excitations, including $e-h$ pair creation and SEE if the electron is near the surface and its energy above the vacuum level. We discuss this matter more thoroughly in Sec. IV.A.

The electron can directly excite $e-h$ pairs too, the decay of which gives rise to the emission of CL and SEs. This produces in general a complex cascade of SEs, which a transport equation approach is suited to model reliably (Rösler and Brauer, 1991). The cascade includes energetic electrons that generate further SEs and CL, adding complexity to this scenario. However, an element of simplicity comes from the separation between coherent and incoherent processes, from the point of view of the emitted light, as explained in Sec. IV. In particular, the coherent CL signal is dominant in metals.

We are interested in low-energy excitations, which involve holes and electrons in the valence and conduction bands, as well as collective modes (e.g., localized plasmons and SPPs). This is by far the most intense part of the loss spectrum (see Fig. 4). Its analysis yields information on the material's optical response with the kind of spatial resolution that is currently desired in the context of nanophotonics. It is our purpose to review historical developments, to summarize recent advances in this area, to present an overview of theoretical methods, and to point out some opportunities opened by electron microscopy in order to expand and complement nanophotonics studies in a way that can be particularly beneficial for emerging areas such as plasmonics. 


\section{INTERACTION OF SWIFT ELECTRONS WITH MATTER}

We first consider some fundamental aspects of the interaction of fast electrons with matter, as well as theoretical approaches suitable to simulate EELS.

\section{A. An evanescent source of light in matter}

The electromagnetic field that accompanies a point charge moving in vacuum can be regarded as an evanescent source of radiation which permits exploring regions of momentum-energy space that lie outside the light cone. This has interesting consequences: fast electrons generate SPPs when passing near a metal surface, as shown in Sec. III.C, but they can also excite nondipolar modes in small particles (Chu et al., 2009), which would be difficult to resolve using external light instead.

It is helpful to examine first the electric field produced by a swift charged particle moving inside a homogeneous medium. The field can be conveniently decomposed into different frequency contributions using the Fourier transform,

$$
\mathbf{E}(\mathbf{r}, t)=\int \frac{d \omega}{2 \pi} \mathbf{E}(\mathbf{r}, \omega) e^{-i \omega t}
$$

and also into momentum components with $\exp (i \mathbf{q} \cdot \mathbf{r})$ spatial dependence. We assume that the electron describes a straight-line trajectory with constant velocity vector $\mathbf{v}$, crossing the origin at time $t=0$. This is a reasonable assumption for the swift electrons and relatively thin specimens typically examined with TEMs. Then the electron charge density becomes $-2 \pi e \delta(\omega-\mathbf{q} \cdot \mathbf{v})$ in $\mathbf{q}$ $-\omega$ space. Direct solution of Maxwell's equations yields

$$
\mathbf{E}(\mathbf{r}, \omega)=\frac{i e}{\pi} \int d^{3} \mathbf{q} \frac{\mathbf{q} / \epsilon-k \mathbf{v} / c}{q^{2}-k^{2} \epsilon} e^{i \mathbf{q} \cdot \mathbf{r}} \delta(\omega-\mathbf{q} \cdot \mathbf{v}),
$$

where $k=\omega / c$ is the light wave number in free space and $\epsilon$ is the dielectric function of the homogeneous medium. It should be noted that $\epsilon$ can depend on both $\mathbf{q}$ and $\omega$. The consequences of the wave-vector dependence are discussed later in Sec. III.B, but they are ignored in the remainder of this section.

Equation (2) contains some interesting elements. Retardation effects show up both through $k^{2} \epsilon$ in the denominator, which modifies the strength of the field (producing, for example, weaker interaction in metals and stronger interaction in low-index dielectrics), and through a term proportional to the velocity vector in the numerator, which adds transverse components to the field.

The delta function inside the integral of Eq. (2) expresses energy conservation for transfers of frequency $\omega$ and wave vector $\mathbf{q}$ from the electron to the material. Neglecting relativistic corrections, the electron energy is reduced from $m_{e} v^{2} / 2$ to $\left|m_{e} \mathbf{v}-\hbar \mathbf{q}\right|^{2} / 2 m_{e}$ during the transfer, so that the energy difference $\hbar \omega$ leads to

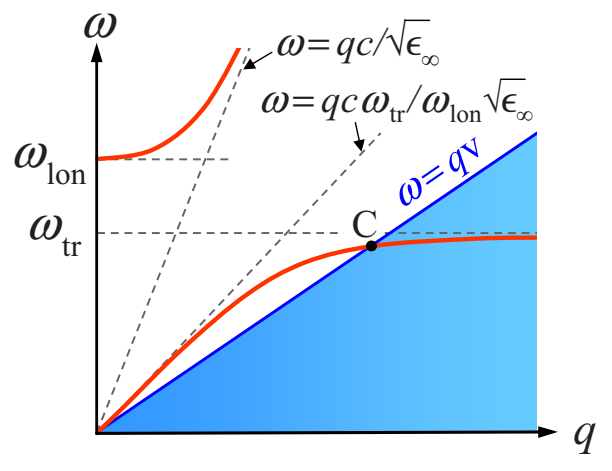

FIG. 5. (Color online) Wave-vector-frequency diagram in a polaritonic material. The diagram shows the light dispersion relation $q^{2}=k^{2} \epsilon$ described by Eq. (A3) (thick solid curves) and its intersection with allowed transfers coming from an electron moving with velocity $\mathbf{v}$ (shaded region). Point $\mathrm{C}$ signals the frequency threshold of Cherenkov radiation (CR) emission.

$$
\omega=\mathbf{q} \cdot \mathbf{v}-\hbar q^{2} / 2 m_{e} \approx \mathbf{q} \cdot \mathbf{v} .
$$

The rightmost expression is the nonrecoil approximation, which works extremely well under the usual condition $\hbar q \ll m_{e} v{ }^{4}$ This approximation remains valid if the energy transfer is computed using relativistic expressions. The kinematically allowed transfers span a solid area for all possible relative orientations between $\mathbf{v}$ and $\mathbf{q}$, as shown by the shaded region in Fig. 5 with upper boundary $\omega=q v$.

The zeros of the denominator in the integrand of Eq. (2) signal the dispersion relation of light in the medium, $q=k \sqrt{\epsilon}$. This has been represented in Fig. 5 for a polaritonic material described by the dielectric function of Eq. (A3) (thick solid curves). The figure shows two different frequency domains separated by the condition $v^{2} \epsilon=c^{2}$ (point $\mathrm{C}$ in Fig. 5 is a graphical solution of this equation). At lower frequencies with respect to $\mathrm{C}$, the electron does not couple to excitations in the medium and the spectral components of the electric field decay exponentially away from the trajectory, as explicitly shown by solving the integral in Eq. (2). We find (Jackson, 1999)

$$
\mathbf{E}(\mathbf{r}, \omega)=\frac{2 e \omega}{v^{2} \gamma_{\epsilon} \epsilon} \mathbf{g}(\mathbf{r}),
$$

where

$$
\mathbf{g}(\mathbf{r})=e^{i \omega z / v}\left[\frac{i}{\gamma_{\epsilon}} K_{0}\left(\frac{\omega R}{v \gamma_{\epsilon}}\right) \hat{\mathbf{z}}-K_{1}\left(\frac{\omega R}{v \gamma_{\epsilon}}\right) \hat{\mathbf{R}}\right],
$$

$\gamma_{\epsilon}=1 / \sqrt{1-\epsilon v^{2} / c^{2}}$ is the Lorentz contraction factor, ${ }^{5}$ and the notation $\mathbf{r}=(\mathbf{R}, z)$ with $\mathbf{R}=(x, y)$ has been employed

\footnotetext{
${ }^{4}$ Electrons with typical TEM energies above $80 \mathrm{keV}$ have velocities $v>0.5 c$. When they undergo valence losses $\hbar \omega$ $<50 \mathrm{eV}$, the momentum transfer is $\hbar q_{z} \approx \hbar \omega / v<0.0004 m_{e} v$ along the direction of the trajectory.

${ }^{5}$ Interestingly, the Lorentz factor $\gamma_{\epsilon}$ involves the velocity of light in the material, $c / \sqrt{\epsilon}$, which is in turn frequency dependent.
} 


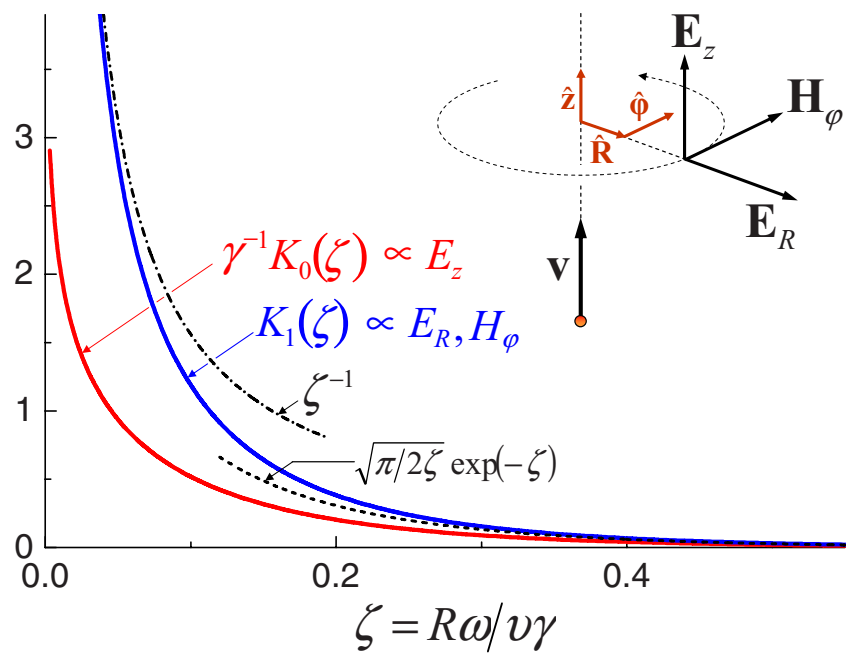

FIG. 6. (Color online) Evanescent character of the electromagnetic field produced by a fast electron. Transverse-spatialdirection dependence of the $\exp (-i \omega t)$ contribution to the electromagnetic field set up by an electron moving in vacuum with velocity $v=0.7 c(\gamma \approx 1.4$ and kinetic energy $\approx 200 \mathrm{keV})$ along the positive $z$ axis. The only nonvanishing components $\left(E_{R}\right.$, $E_{z}$, and $H_{\varphi}$ ) decay exponentially at large distance $R$ from the trajectory. The inset shows the orientation of these components relative to the electron velocity vector. The small- $R$ limit is dominated by the $1 / R$ divergence of $E_{R}$ and $H_{\varphi}$.

(see the inset of Fig. 6), with the velocity taken along $\hat{\mathbf{z}}^{6}$ Similarly, the magnetic field reduces to

$$
\mathbf{H}(\mathbf{r}, \omega)=-\frac{2 e \omega}{v c \gamma_{\epsilon}} K_{1}\left(\frac{\omega R}{v \gamma_{\epsilon}}\right) e^{i \omega z / v} \hat{\varphi},
$$

where $\hat{\varphi}$ is the azimuthal unit vector (see Fig. 6). The announced exponential decay with $R$ of both $\mathbf{E}(\mathbf{r}, \omega)$ and $\mathbf{H}(\mathbf{r}, \omega)$ arises from the asymptotic behavior of the modified Bessel functions $K_{m}$ for large arguments (Abramowitz and Stegun, 1972). The nonvanishing components of these fields are shown in Fig. 6. The electromagnetic field extends up to distances of the order of $\sim v \gamma_{\epsilon} / \omega$, the Bohr cutoff. Notice, however, that one cannot assign this value to a characteristic Coulomb delocalization distance since the field diverges at the origin as $\sim 1 / R$, so that large interaction contrast is expected across small distances in the region close to the trajectory.

When the electron moves faster than light in the medium, under the condition

$$
v>c / \sqrt{\epsilon}
$$

there is overlap between the photon dispersion relation and the shaded region in Fig. 5, so that the electron can emit Cherenkov radiation (CR) (see Sec. IV.D). The field displays oscillatory behavior and decays as $1 / \sqrt{R}$

\footnotetext{
${ }^{6}$ The square roots are chosen to yield positive real parts in this work. Notice that $\operatorname{Im}\{\epsilon\}$ is always positive in the retarded response formalism followed here, and it becomes a positive infinitesimal in nonlossy dielectrics.
}

away from the trajectory [this stems from the modified Bessel functions $K_{0}$ and $K_{1}$ for imaginary argument in Eq. (5)].

It should be stressed that Eqs. (4)-(6) and Fig. 6 refer to each monochromatic component of the electromagnetic field, evolving with time as $\exp (-i \omega t)$ [see Eq. (1)]. An electron moving in vacuum can actually be regarded as an evanescent source of supercontinuum light with the spectral dependence shown in Fig. 6.

\section{B. Classical dielectric formalism}

The pioneering work of Fermi (1940) on the stopping of fast charged particles in dielectric materials opened up the application of classical electrodynamics to describe the interaction of swift electrons with matter. Following this useful tradition, we now extend the dielectric formalism of the previous section to cope with inhomogeneous samples and discuss in particular the spectral loss probability, which is relevant to EELS experiments.

The energy loss suffered by a fast electron moving with constant velocity $\mathbf{v}$ along a straight-line trajectory $\mathbf{r}=\mathbf{r}_{e}(t)$ can be related to the force exerted by the induced electric field $\mathbf{E}^{\text {ind }}$ acting back on the electron as (Ritchie, 1957)

$$
\Delta E=e \int d t \mathbf{v} \cdot \mathbf{E}^{\text {ind }}\left[\mathbf{r}_{e}(t), t\right]=\int_{0}^{\infty} \hbar \omega d \omega \Gamma_{\mathrm{EELS}}(\omega),
$$

where the $-e$ electron charge has been included (i.e., $\Delta E>0)$ and

$$
\Gamma_{\operatorname{EELS}}(\omega)=\frac{e}{\pi \hbar \omega} \int d t \operatorname{Re}\left\{e^{-i \omega t} \mathbf{v} \cdot \mathbf{E}^{\mathrm{ind}}\left[\mathbf{r}_{e}(t), \omega\right]\right\}
$$

is the so-called loss probability, which is given per unit of transferred frequency $\omega$. The problem of calculating the loss probability reduces then to solving the electric field set up by the electron. A great deal of work has been devoted to obtaining the electric field for many geometries, including planar surfaces, isolated spheres, neighboring spheres, circular cylinders, wedges, and more complex shapes, using both analytical and fully numerical methods, either within the nonretarded approximation, based on solutions of Poisson's equation, or with full inclusion of retardation effects by solving Maxwell's equations (see Sec. III, and references therein). Next, we outline the general features of this formalism.

\section{Nonretarded approximation}

In the nonretarded approximation, we neglect the delay experienced by the electromagnetic signal that mediates the electron-sample interaction. Then, the electric field admits the form $\mathbf{E}(\mathbf{r}, \omega)=-\nabla \phi(\mathbf{r}, \omega)$, and we can disregard $\mathbf{H}$ in the absence of magnetic response.

It is useful to express the electric potential $\phi$ in terms of the screened interaction $W\left(\mathbf{r}, \mathbf{r}^{\prime}, \omega\right)$, defined as the potential created at $\mathbf{r}$ by a unit point charge located at $\mathbf{r}^{\prime}$ [an implicit $\exp (-i \omega t)$ time dependence is understood]. This quantity has to be combined with the charge den- 
sity corresponding to the moving electron. Considering a nonrecoiled straight-line trajectory and assuming without loss of generality that the velocity vector is directed along the positive $z$ axis [i.e., the trajectory is $\mathbf{r}_{e}(t)=\mathbf{r}_{0}$ $+\mathbf{v} t$ and $\mathbf{v}=v \hat{\mathbf{z}}]$, the electron charge density in frequency space $\omega$ reduces to

$$
\begin{aligned}
\rho(\mathbf{r}, \omega) & =-e \int d t e^{i \omega t} \delta\left(\mathbf{r}-\mathbf{r}_{0}-\mathbf{v} t\right) \\
& =-\frac{e}{v} \delta\left(\mathbf{R}-\mathbf{R}_{0}\right) e^{i \omega\left(z-z_{0}\right) / v},
\end{aligned}
$$

where the notation shown in the inset of Fig. 6 has been used. From here, the potential reads

$$
\phi(\mathbf{r}, \omega)=-\frac{e}{v} \int d z^{\prime} W\left(\mathbf{r}, \mathbf{R}_{0}, z^{\prime}, \omega\right) e^{i \omega\left(z^{\prime}-z_{0}\right) / v} .
$$

Finally, substituting these expressions into Eq. (8), the nonretarded (NR) loss probability is

$$
\begin{aligned}
\Gamma_{\mathrm{EELS}}^{\mathrm{NR}}\left(\mathbf{R}_{0}, \omega\right)= & \frac{e^{2}}{\pi \hbar v^{2}} \int d z d z^{\prime} \cos \left[\frac{\omega\left(z-z^{\prime}\right)}{v}\right] \\
& \times \operatorname{Im}\left\{-W\left(\mathbf{R}_{0}, z, \mathbf{R}_{0}, z^{\prime}, \omega\right)\right\},
\end{aligned}
$$

where the dependence of $\Gamma_{\mathrm{EELS}}^{\mathrm{NR}}$ on the electron impact parameter $\mathbf{R}_{0}$ is explicitly shown. ${ }^{7,8}$

The loss probability can be thus derived from the knowledge of the frequency-dependent screened interaction $W$. A local description of the sampled materials in terms of a frequency- and space-dependent dielectric function $\epsilon(\mathbf{r}, \omega)$ often provides reliable results. Detailed expressions of $W$ are given in Appendix B for planar, spherical, and cylindrical geometries. Nevertheless, Eq. (10) is valid beyond the local response approximation, and subtle effects such as local field corrections can be incorporated via more realistic quantum-mechanical representations of the screened interaction (see Sec. V.E).

\section{Retardation effects}

In high-voltage TEMs, retardation may become important because the speed of the charged projectiles is a sizable fraction of the speed of light. For instance, the Lorentz contraction factor at $200 \mathrm{keV}$ takes already a value significantly different from $1, \gamma=1.4$. Retardation has two noticeable consequences for spectroscopy: (1) it increases the range of interaction of the electron probe in directions normal to the trajectory, as clearly shown by Eq. (5) and Fig. 6 and (2) it produces redshifts in excitation mode energies (Myroshnychenko, Rodríguez-

\footnotetext{
${ }^{7}$ It should be noted that the reciprocity theorem $\left[W\left(\mathbf{r}, \mathbf{r}^{\prime}, \omega\right)\right.$ $\left.=W\left(\mathbf{r}^{\prime}, \mathbf{r}, \omega\right)\right]$ and the fact that the bare Coulomb interaction is a real function have been utilized in the derivation of Eq. (10).

${ }^{8}$ The induced field in Eq. (8) can be safely replaced by the total field because the bare field of the moving charge does not produce stopping. We have accordingly dropped the superscript "ind" in Eq. (10).
}

Fernández, et al., 2008). The latter are discussed below in further detail, but we can anticipate that retardation turns out to be important when the excitations extend over specimen distances that cannot be neglected compared with the corresponding light wavelength. A proper description of these effects requires calculating the electric field of Eq. (8) from Maxwell's equations in the presence of the moving electron and the sample under consideration.

The electromagnetic response of a structured material is fully captured in its electric Green's tensor. In particular, the electric field produced by an external current density $\mathbf{j}(\mathbf{r}, \omega)$ in an inhomogeneous medium of permittivity $\epsilon(\mathbf{r}, \omega)$ can be written in frequency space $\omega$ as

$$
\mathbf{E}(\mathbf{r}, \omega)=-4 \pi i \omega \int d^{3} \mathbf{r}^{\prime} G\left(\mathbf{r}, \mathbf{r}^{\prime}, \omega\right) \cdot \mathbf{j}\left(\mathbf{r}^{\prime}, \omega\right)
$$

in terms of $G$, the electric Green's tensor of Maxwell's equations in Gaussian units, satisfying

$$
\begin{aligned}
\boldsymbol{\nabla} & \times \boldsymbol{\nabla} \times G\left(\mathbf{r}, \mathbf{r}^{\prime}, \omega\right)-k^{2} \epsilon(\mathbf{r}, \omega) G\left(\mathbf{r}, \mathbf{r}^{\prime}, \omega\right) \\
& =-\frac{1}{c^{2}} \delta\left(\mathbf{r}-\mathbf{r}^{\prime}\right)
\end{aligned}
$$

and vanishing far away from the sources $\left(\left|\mathbf{r}-\mathbf{r}^{\prime}\right| \rightarrow \infty\right.$ limit).

For the electron charge density of Eq. (9), the external current density reduces to $\mathbf{j}=\mathbf{v} \rho$, which upon insertion into Eq. (11), and this in turn into Eq. (8), allows us to write the loss probability as

$$
\begin{aligned}
\Gamma_{\mathrm{EELS}}\left(\mathbf{R}_{0}, \omega\right)= & \frac{4 e^{2}}{\hbar} \int d z d z^{\prime} \cos \left[\frac{\omega\left(z-z^{\prime}\right)}{v}\right] \\
& \times \operatorname{Im}\left\{-G_{z z}\left[\mathbf{R}_{0}, z, \mathbf{R}_{0}, z^{\prime}, \omega\right]\right\},
\end{aligned}
$$

where $G_{z z}=\hat{\mathbf{z}} \cdot G \cdot \hat{\mathbf{z}} .^{9}$ Interestingly, this expression works for any sign of $v$, and therefore the loss probability is independent of whether the electron moves toward positive or negative $z$ 's.

\section{Quantum approach}

The quantum nature of both the electron probe and the excitations sustained by the targeted materials permeate many aspects of the electron-sample interaction. However, Ritchie and Howie (1988) showed that a

\footnotetext{
${ }^{9}$ In the retarded case, the reciprocity theorem states that $G\left(\mathbf{r}, \mathbf{r}^{\prime}, \omega\right)=G^{T}\left(\mathbf{r}^{\prime}, \mathbf{r}, \omega\right)$, a fact that we have used to recast exponential factors involving $z$ and $z^{\prime}$ into a cosine function in the derivation of Eq. (13). Moreover, the free-space Green's function is entirely made of plane-wave components lying inside the light cone, so that it cannot contain wave vectors $\omega / v>k$. This guarantees that the integral in Eq. (13) yields zero for an electron moving in vacuum and that we are allowed to utilize the total rather than the induced field to obtain this equation.
} 
quantum-mechanical description of EELS yields the same results as a semiclassical formalism if all the inelastic signal is collected.

We work for simplicity in the nonretarded limit, in which the validity of Eq. (10) is demonstrated next under very common experimental conditions (Ritchie and Howie, 1988). A generalization to include retardation is also offered.

\section{Quantum description of the target}

The last century has witnessed important developments in the field of interaction of fast charges with solids, particularly in the community of electronic and atomic collisions [see, for instance, Palmer and Rous (1992), Ziegler (1999), Winter (2002), and references therein], from which our theoretical understanding of electron microscopy has benefited considerably. In that context, ion and electron stopping was important to understand dynamical screening in solids, with interesting developments such as the description of plasmon excitations using second quantization schemes in planar ( $\mathrm{Lu}-$ cas and Šunjić, 1971) and spherical (Ashley and Ferrell, 1976) surfaces. Moreover, a nonretarded fully quantummechanical expression for the loss probability was derived using a self-energy formalism (Echenique et al., 1987, 1990). A quantum treatment of the target has been shown to be necessary for a correct assessment of delocalization in the excitation of core levels (Oxley and Allen, 1998; Allen et al., 2003). We obtain here a general expression for the loss probability, starting from the more widely used Fermi golden rule, and assess the conditions under which it agrees with the semiclassical formalism presented above, as discussed by Ritchie and Howie (1988).

During the interaction of a fast electron with a target, the latter can undergo transitions from its ground state $|0\rangle$ of energy $\hbar \omega_{0}$ to excited states $|n\rangle$ of energy $\hbar \omega_{n}$, while the incoming electron of energy $\hbar \varepsilon_{i}$ and wave function $\psi_{i}(\mathbf{r})$ acquires components $\psi_{f}(\mathbf{r})$ of lower energy $\hbar \varepsilon_{\text {f. }}$. Since the interaction with very energetic electrons is generally small, the transition rate is well described within first-order perturbation theory (Fermi's golden rule),

$$
\begin{aligned}
\frac{d \Gamma^{\mathrm{NR}}}{d t}= & \frac{2 \pi e^{4}}{\hbar^{2}} \sum_{f, n}\left|\int d^{3} \mathbf{r} d^{3} \mathbf{r}^{\prime} \frac{\psi_{f}^{*}(\mathbf{r}) \psi_{i}(\mathbf{r})\left\langle n\left|\hat{\rho}\left(\mathbf{r}^{\prime}\right)\right| 0\right\rangle}{\left|\mathbf{r}-\mathbf{r}^{\prime}\right|}\right|^{2} \\
& \times \delta\left(\varepsilon_{f}-\varepsilon_{i}+\omega_{n}-\omega_{0}\right),
\end{aligned}
$$

where we have used the target-probe Coulomb interaction and $\hat{\rho}$ is the target electron-density operator (see Fig. 7). We can now recast Eq. (14) into a more convenient form by relating the target matrix elements to the linear-response susceptibility (Pines and Nozières, 1966)

$$
\begin{aligned}
\operatorname{Im}\left\{\chi\left(\mathbf{r}, \mathbf{r}^{\prime}, \omega\right)\right\}= & -\frac{\pi e^{2}}{\hbar} \sum_{n}\langle 0|\hat{\rho}(\mathbf{r})| n\rangle\left\langle n\left|\hat{\rho}\left(\mathbf{r}^{\prime}\right)\right| 0\right\rangle \\
& \times \delta\left(\omega_{n}-\omega_{0}-\omega\right),
\end{aligned}
$$

valid for $\omega>0$, and in turn to the screened interaction

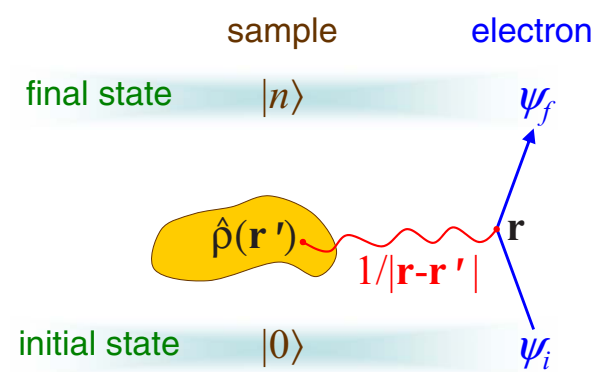

FIG. 7. (Color online) Schematic representation of the Coulomb interaction between a swift electron and a specimen, showing the elements involved in Eq. (14), including the target electron-density operator $\hat{\rho}$ at point $\mathbf{r}^{\prime}$.

$$
W^{\text {ind }}\left(\mathbf{r}, \mathbf{r}^{\prime}, \omega\right)=\int d^{3} \mathbf{r}_{1} d^{3} \mathbf{r}_{2} \frac{\chi\left(\mathbf{r}_{1}, \mathbf{r}_{2}, \omega\right)}{\left|\mathbf{r}-\mathbf{r}_{1}\right|\left|\mathbf{r}^{\prime}-\mathbf{r}_{2}\right|} .
$$

Then, the sum of Eq. (14) can be separated into specific values of the frequency transfer $\omega=\varepsilon_{i}-\varepsilon_{f}$ as

$$
\frac{d \Gamma^{\mathrm{NR}}}{d t}=\int_{0}^{\infty} d \omega \frac{d \Gamma^{\mathrm{NR}}(\omega)}{d t}
$$

where

$$
\begin{aligned}
\frac{d \Gamma^{\mathrm{NR}}(\omega)}{d t}= & \frac{2 e^{2}}{\hbar} \sum_{f} \int d^{3} \mathbf{r} d^{3} \mathbf{r}^{\prime} \psi_{f}(\mathbf{r}) \psi_{i}^{*}(\mathbf{r}) \psi_{f}^{*}\left(\mathbf{r}^{\prime}\right) \psi_{i}\left(\mathbf{r}^{\prime}\right) \\
& \times \operatorname{Im}\left\{-W\left(\mathbf{r}, \mathbf{r}^{\prime}, \omega\right)\right\} \delta\left(\varepsilon_{f}-\varepsilon_{i}+\omega\right) .
\end{aligned}
$$

This expression is general for incident electrons of well-defined energy, where $W$ contains all quantummechanical details of the sample response, although expressions obtained from dielectric theory such as those offered in Appendix B yield reliable results in most situations encountered in practice.

The generalization of Eq. (15) to include retardation is obtained either from an extension of the above formalism (using current-current response functions) or by dealing with the quantized photon field (see García de Abajo and Kociak, 2008a). One finds

$$
\begin{aligned}
\frac{d \Gamma(\omega)}{d t}= & \frac{8 \pi \hbar e^{2}}{m_{e}^{2}} \sum_{f} \int d^{3} \mathbf{r} d^{3} \mathbf{r}^{\prime} \psi_{f}(\mathbf{r}) \psi_{f}^{*}\left(\mathbf{r}^{\prime}\right) \\
& \times\left[\nabla \psi_{i}^{*}(\mathbf{r})\right] \cdot \operatorname{Im}\left\{-G\left(\mathbf{r}, \mathbf{r}^{\prime}, \omega\right)\right\} \cdot\left[\nabla \psi_{i}\left(\mathbf{r}^{\prime}\right)\right] \\
& \times \delta\left(\varepsilon_{f}-\varepsilon_{i}+\omega\right),
\end{aligned}
$$

where $G$ is the Green tensor defined in Eq. (11).

\section{Quantum effects in the fast electrons}

Several researchers have analyzed the formation of images in electron microscopes from a quantummechanical viewpoint, considering the influence of instrumental parameters, such as, for example, the beam aperture and the collection angle (Kohl, 1983; Batson, 1985; Ritchie and Howie, 1988; Muller and Silcox, 1995). Particular emphasis has been laid on dealing with delocalization, which is relevant for devising ways of improving the spatial resolution of the inelastic signal (Oxley 
and Allen, 1998; Allen et al., 2003). We are not going to enter into details of the instruments here, but it is instructive to consider effects related to partial detection of the transmitted electrons.

We can regard each electron in a TEM as consisting of a coherent superposition of plane waves, yielding for instance a narrow focus close to the sample in STEM mode. An accurate approximation can be adopted if we keep in mind that the angular aperture of the beam has remained in the range of a few milliradians since the early days of electron microscopy (Ruska, 1987). The components of the incident electron wave vector perpendicular to the beam direction $z$ are thus $p_{i \perp} \leq 10^{-2} p_{i}$, where $p_{i}$ is the total wave vector. The parallel components become $p_{i \|}=\left(p_{i}^{2}-p_{i \perp}^{2}\right)^{1 / 2} \approx p_{i}$, where we are neglecting $\approx p_{i \perp}^{2} / 2 p_{i} \leq 10^{-4} p_{i}$ compared with $p_{i}$. We conclude that the incident charge can be reliably described near the sample by the wave function

$$
\psi_{i}(\mathbf{r})=\frac{1}{L^{1 / 2}} e^{i p_{i} z} \psi_{i \perp}(\mathbf{R})
$$

where $L$ is the quantization length of the trajectory and the $\mathbf{R}$ dependence reflects the variation perpendicular to $z$. This should be valid for typical sample thickness $\Delta z$ $\sim 50 \mathrm{~nm}$, along which the lateral divergence of the beam can be quantified as $\Delta R \leqq 0.5 \mathrm{~nm}$, a small value compared to typical dimensions of common photonic and plasmonic structures.

The transmitted electrons can again be described by plane waves of wave vectors $\mathbf{p}_{f}$. Then, the frequency transfer reduces to $\omega \approx \mathbf{q} \cdot \mathbf{v}$ under the approximation of Eq. (3), where $\mathbf{q}=\mathbf{p}_{i}-\mathbf{p}_{f}$ is the wave-vector transfer and $\mathbf{v}=\left(\hbar / m_{e}\right) p_{i} \hat{\mathbf{z}}$ is the incident electron velocity.

We are now prepared to recast Eq. (15) into a more practical formula. Using delta-function normalization for the final states, multiplying the transition rate by the interaction time $L / v$, and making use of the above considerations, we find

$$
\Gamma^{\mathrm{NR}}(\omega)=\int d^{2} \mathbf{p}_{f \perp} \frac{d \Gamma^{\mathrm{NR}}(\omega)}{d \mathbf{p}_{f \perp}},
$$

where

$$
\begin{aligned}
\frac{d \Gamma^{\mathrm{NR}}(\omega)}{d \mathbf{p}_{f \perp}}= & \frac{e^{2}}{4 \pi^{3} \hbar v^{2}} \int d^{3} \mathbf{r} d^{3} \mathbf{r}^{\prime} \psi_{i \perp}^{*}(\mathbf{R}) \psi_{i \perp}\left(\mathbf{R}^{\prime}\right) \\
& \times e^{i \mathbf{p}_{f \perp} \cdot\left(\mathbf{R}-\mathbf{R}^{\prime}\right)} e^{i \omega\left(z^{\prime}-z\right) / v} \operatorname{Im}\left\{-W\left(\mathbf{r}, \mathbf{r}^{\prime}, \omega\right)\right\}
\end{aligned}
$$

is the loss probability resolved in $\mathbf{p}_{f \perp}$ (the lateral wave vector of the transmitted electron $\perp \mathbf{v}$ ) or, equivalently, the outgoing direction. This formula indicates that STEMs can be used to retrieve the full nonlocal dependence of $\operatorname{Im}\left\{-W\left(\mathbf{r}, \mathbf{r}^{\prime}, \omega\right)\right\}$, but we defer a detailed discussion of this point to Sec. V.B.2, in which we explore vicinage effects.

In practice, electron beams are polychromatic. However, a trivial extension of the above formalism for typical beams with random phases between different energy components leads to the intuitive result that the loss probability is the average of Eq. (17) over the incident beam spectrum.

Equation (17) leads to a powerful result, established by Ritchie and Howie (1988), regarding the validity of the classical dielectric formalism employed in Sec. II.B.1. The unrestricted integral over all possible values of $\mathbf{p}_{f \perp}$ yields $\delta\left(\mathbf{R}-\mathbf{R}^{\prime}\right)$, so that the loss probability reduces to

$$
\Gamma_{\mathrm{EELS}}^{\mathrm{NR}}(\omega)=\int d^{2} \mathbf{R}\left|\psi_{i \perp}(\mathbf{R})\right|^{2} \Gamma_{\mathrm{EELS}}^{\mathrm{NR}}(\mathbf{R}, \omega),
$$

where $\Gamma_{\mathrm{EELS}}^{\mathrm{NR}}(\mathbf{R}, \omega)$ is given by Eq. (10). In other words, the EELS probability is well described by Poisson's equation if all the inelastic signal is collected (i.e., by use of a wide acceptance angle in the spectrometer), but it needs to be averaged over electron impact parameters weighted by the spot intensity $\left|\psi_{i \perp}(\mathbf{R})\right|^{2}$.

Using the retarded expression given above for $d \Gamma(\omega) / d t$, and noticing that $\boldsymbol{\nabla} \psi_{i}(\mathbf{r}) \approx \psi_{i}(\mathbf{r}) i p_{i} \hat{\mathbf{z}}$, the retarded generalization of Eq. (17) reduces to

$$
\begin{aligned}
\frac{d \Gamma(\omega)}{d \mathbf{p}_{f \perp}}= & \frac{e^{2}}{\pi^{2} \hbar} \int d^{3} \mathbf{r} d^{3} \mathbf{r}^{\prime} \psi_{i \perp}^{*}(\mathbf{R}) \psi_{i \perp}\left(\mathbf{R}^{\prime}\right) \\
& \times e^{i \mathbf{p}_{f \perp} \cdot\left(\mathbf{R}-\mathbf{R}^{\prime}\right)} e^{i \omega\left(z^{\prime}-z\right) / v} \operatorname{Im}\left\{-G_{\mathbf{z z}}\left(\mathbf{r}, \mathbf{r}^{\prime}, \omega\right)\right\} .
\end{aligned}
$$

From here, integrating over $\mathbf{p}_{f \perp}$, one obtains

$$
\Gamma_{\mathrm{EELS}}(\omega)=\int d^{2} \mathbf{R}\left|\psi_{i \perp}(\mathbf{R})\right|^{2} \Gamma_{\mathrm{EELS}}(\mathbf{R}, \omega),
$$

where $\Gamma_{\text {EELS }}(\mathbf{R}, \omega)$ is the same as in Eq. (13), thus validating the classical retarded formalism when all inelastic losses are recorded.

\section{ELECTRON ENERGY-LOSS SPECTROSCOPY}

Hillier and Baker (1944) were the first to propose and demonstrate EELS in TEMs, although earlier pioneering experiments reported energy losses of transmitted electrons in thin films (Leithäuser, 1904). This technique has become standard in the electron microscopy community and is capable of providing information on electronic band structures and plasmons in the low-energyloss region, as well as atomically resolved chemical identity encoded in core losses (Browning et al., 1993). During its prolific existence, valence EELS has contributed to fields as varied as biochemistry [for example, in the study of excitations sustained by nucleic acid bases reported by Crewe et al. (1971)] interplanetary science [for instance, in the explanation of a $5 \mathrm{eV}$ strong absorption feature in cosmic dust found by Bradley et al. (2005)] and microelectronics [in particular, in the investigation of the resistivity of CMOS elements performed by Pokrant et al. (2006)].

Since the early days of EELS, transmission electron microscopes have undergone a tremendous series of improvements that currently permit achieving $\sim 0.1 \mathrm{eV}$ energy resolution for a subnanometer-size electron beam. This opens up new vistas in the low-energy-loss region, 
such as addressing the optical properties of photonic structures with unprecedented spatial detail.

The excitation spectrum of a typical material is shown in Fig. 4 for bulk Ag. The EELS intensity for a given energy loss $\hbar \omega$ directly reflects the strength of specimen modes corresponding to that energy (Egerton, 1996). The loss spectrum has been traditionally separated into valence- and core-loss regions, with the division between them arbitrarily established at $\sim 50 \mathrm{eV}$. With the customary use of thin samples, the main feature in an EELS spectrum is the zero-loss peak (ZLP) of unscattered electrons, in which unresolved very-low-energy excitations (e.g., phonons) are buried. The intensity of valence features is over two orders of magnitude lower than the ZLP in the $20 \mathrm{~nm} \mathrm{Ag} \mathrm{film} \mathrm{considered} \mathrm{in} \mathrm{Fig.} \mathrm{4,} \mathrm{while}$ higher-energy core excitations are even less probable. This presents a typical scenario in which the inelastic signal is superimposed into the tail of the ZLP. Removal of the ZLP is thus important, and various sophisticated procedures have been devised that produce reliable results (Lazar et al., 2006; Nelayah, Kociak, et al., 2007).

Valence losses are generally more intense than core losses and allow any given amount of inelastic signal to be collected with less electron irradiation, therefore producing minimum damage to the sample. However, the interpretation of low-energy-loss images is less direct because it involves the excitation of delocalized modes (Howie, 2003; Erni et al., 2008). In this respect, it is useful to rely on theoretical simulations, which are now advanced to the point of dealing with virtually any sample geometry in a predictive way.

Raether (1980) and Egerton (1996) works on EELS cover these subjects and provide detailed considerations of the operation and principles of TEMs. Shorter summaries (Brydson, 2001) and collections of experimental spectra (Ahn, 2004) have been presented. Comprehensive reports on valence EELS (Wang, 1996) and its theoretical analysis (Rivacoba et al., 2000) are also available. The present article intends to supplement this field by providing a more extended overview of low-energy losses, their characteristics and theoretical understanding, and several examples of novel application to nanophotonics, for which they might become particularly useful.

\section{A. Space, momentum, and energy resolution}

Nowadays, some commercial TEMs incorporate the latest achievements in space and energy resolution. The former has been much improved over the last decade by the development of aberration correctors in the electron optics, particularly the spherical aberration (Uhlemann and Haider, 1998). This has reduced the size of the beam spots from the 1-2 $\AA$, commonly achieved without such correctors, down to subangstrom dimensions (Krivanek et al., 1999), which allows, for instance, the imaging of closely spaced atoms in crystal samples using core losses (Batson et al., 2002; Nellist et al., 2004; Varela et al., 2004, 2007) and even identifying details of chemical bonding (Muller et al., 2008). However, the interpretation of atomically resolved images is sometimes difficult and requires reliance on simulations (Oxley and Pennycook, 2008; D'Alfonso, Wang, et al., 2008). The search for improved spatial resolution through aberration correction is actually a work in progress, for example, through theoretical analysis for improved incoherent imaging (Intaraprasonk et al., 2008) and through new subangstrom beam characterization techniques (Dwyer et al., 2008).

The factors that limit the spatial resolution of a microscope are typically known as delocalization effects (Egerton, 2003, 2007, 2009; Erni and Browning, 2005). There are several of them, of very different nature, which can be grouped into three distinct categories: (1) instrumental or lens delocalization, which as noted above can be reduced below $1 \AA$; (2) Coulomb delocalization associated with the finite range of the field that accompanies a fast electron (see Fig. 6 and discussion in Sec. II.A); and (3) the extended nature of the excitations that are probed, varying from macroscopic distances in the case of low-energy SPPs and CR losses to nanometers in particle plasmons and less than $1 \AA$ for core losses. For example, subatomic resolution is currently limited by the size of the probe rather than by the extension of the ionizing interaction that is employed to resolve core levels (Allen et al., 2003).

Additionally, spectral resolution has dramatically improved with the arrival of new spectrometers yielding $\sim 50 \mathrm{meV}$ accuracy (Brink et al., 2003) and electron monochromators (Su et al., 2003), which roughly consist in filtering out incident electrons outside a narrow energy window at the expense of reducing the beam current. An energy resolution of $0.1 \mathrm{eV}$ has been achieved. Measuring excitations down to less than $\hbar \omega=0.5 \mathrm{eV}$ is now possible, thanks to the limited extension of the monochromatized ZLP (Terauchi et al., 1999; Lazar et al., 2006). This spectral resolution should be sufficient for studying most collective excitations supported by metallic systems, in which the intrinsic width produced by absorption is generally larger than $0.1 \mathrm{eV}$.

Deconvolution techniques, utilized to eliminate the ZLP and enhance spectral resolution, have progressed in recent years (van Benthem et al., 2001; Lazar et al., 2006). Furthermore, dynamical instabilities in the electron beam energy, which directly damage spectral details, are now corrected by resorting to fast spectral acquisition, well above the $50-60 \mathrm{~Hz}$ of the omnipresent electrical network, and by subsequent addition of the collected spectra after repositioning the ZLP maximum (Nelayah, Kociak, et al., 2007).

A reasonable degree of momentum-transfer resolution is possible in TEMs by varying the divergence half-angle of the incident beam $\varphi_{\text {in }}$ and the collection half-angle of the spectrometer $\varphi_{\text {out }}$ [see Fig. 2(a)]. These parameters are typically in the range of a few milliradians. Of course, the radius of the electron spot $\Delta R$, which controls spatial resolution in STEMs, is related to $\varphi_{\text {in }}$ through the uncertainty principle, $\left(m_{e} / \hbar\right) \Delta R \varphi_{\text {in }} \geqslant 1 / 2 v$. Actual operation conditions in TEMs are very close to this limit. 
These impressive achievements are the result of a historical quest, still in progress, for ever better spatial and spectral resolution (Egerton, 2003, 2007; Erni and Browning, 2005). However, many factors are remaining that limit the performance of electron microscopes, as recently reviewed by Egerton (2007).

\section{B. Bulk losses and determination of bulk dielectric functions}

Although the volume of homogeneous materials offers little chances for performing microscopy, bulk losses are a splendid source of information on optical properties (Raether, 1980; Palik, 1985) and they configure an area of research in which the electron microscopy community has made important contributions. In a homogeneous medium, we can combine Eqs. (2) and (8) to write the EELS probability as

$$
\Gamma_{\text {bulk }}(\omega)=\frac{e^{2} L}{\pi \hbar v^{2}} \operatorname{Im}\left\{\left(\frac{v^{2}}{c^{2}}-\frac{1}{\epsilon}\right) \ln \left(\frac{q_{c}^{2}-k^{2} \epsilon}{(\omega / v)^{2}-k^{2} \epsilon}\right)\right\},
$$

where $L$ is the length of the trajectory and $\epsilon$ has been assumed to be independent of wave vector $q$. This is the local response approximation, which applies when low enough momentum transfers are collected below a certain cutoff

$$
\hbar q_{c} \approx \sqrt{\left(m_{e} v \varphi_{\mathrm{out}}\right)^{2}+(\hbar \omega / v)^{2}}
$$

that is determined by the half-aperture collection angle of the microscope spectrometer $\varphi_{\text {out }}$ (assuming $\varphi_{\text {in }}$ $\left.\ll \varphi_{\text {out }}\right)$.

The nonretarded version ( $c \rightarrow \infty$ limit) of Eq. (18),

$$
\Gamma_{\text {bulk }}^{\mathrm{NR}}(\omega)=\frac{2 e^{2} L}{\pi \hbar v^{2}} \operatorname{Im}\left\{-\frac{1}{\epsilon}\right\} \ln \left(q_{c} v / \omega\right),
$$

is commonly employed to perform Kramers-Kronig (KK) analysis and to retrieve bulk optical constants from measured EELS data after absolute calibration of the loss function $\operatorname{Im}\{-1 / \epsilon\}$ (Shiles et al., 1980; Pflüger and Fink, 1991; Egerton, 1996; French et al., 1998; Zhang et al., 2008). However, retardation corrections such as CR losses (see Sec. IV.D) can be very damaging in the determination of electronic band gaps and they must be carefully removed from the measured spectra in some cases (Jouffrey et al., 2004; Stöger-Pollach et al., 2006; StögerPollach and Schattschneider, 2007; Erni and Browning, 2008). In addition, multiple inelastic scattering events and factors depending on beam divergence and spectrometer acceptance angles, as well as the precision in the alignment of the sample, have to be properly addressed before using KK analysis to retrieve $\epsilon$ (Bertoni and Verbeeck, 2008; Stöger-Pollach, 2008). Separation of volume and surface losses adds another complication, which can be solved by comparing spectra acquired from
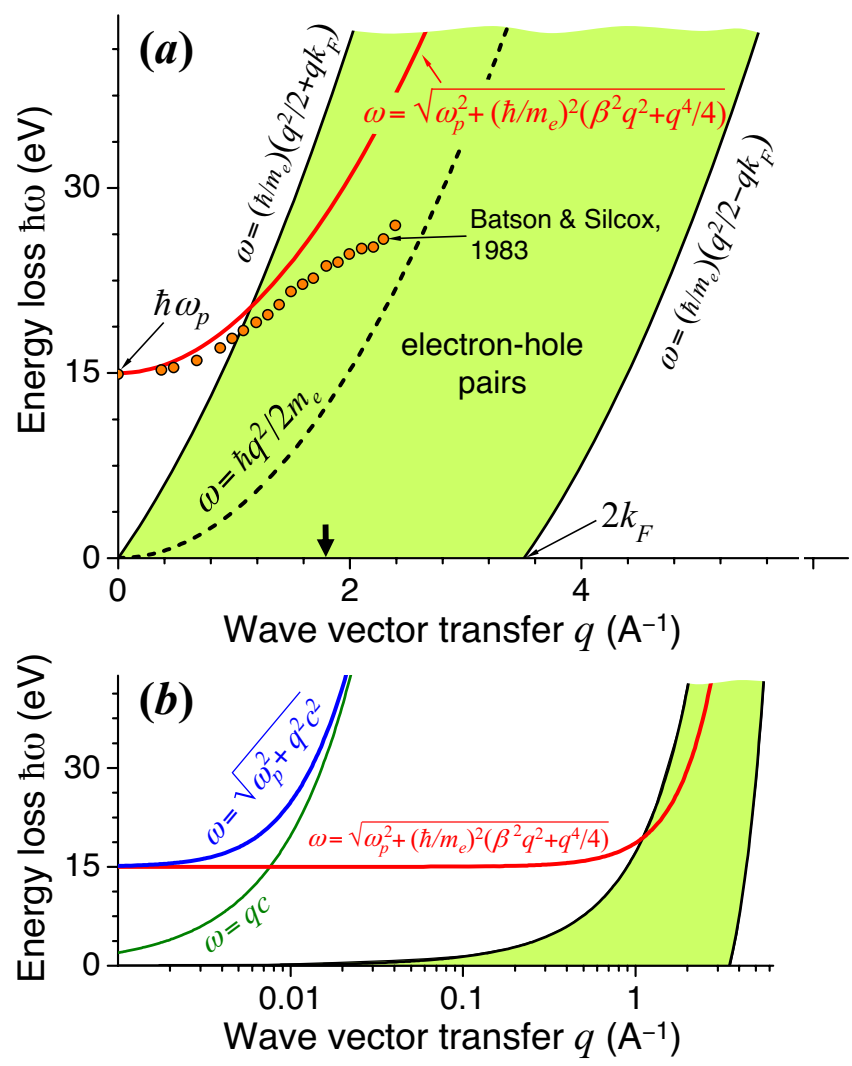

FIG. 8. (Color online) Bulk excitations in aluminum. (a) The conduction band of $\mathrm{Al}$ is well described by a degenerate freeelectron gas of Fermi wave vector $k_{F}=1.75 \AA^{-1}$. The $e-h$ pair excitations supported by this material are contained in the shaded region within the plotted wave-vector-energy $(q-\hbar \omega)$ diagram. The volume plasmon dispersion relation measured by Batson and Silcox (1983) (symbols) is compared to the longitudinal plasmon-pole approximation $\omega=\left[\omega_{p}^{2}+\left(\hbar / m_{e}\right)^{2}\left(\beta^{2} q^{2}\right.\right.$ $\left.\left.+q^{4} / 4\right)\right]^{1 / 2}$ (thick solid curve) derived from the condition $\epsilon_{\text {lon }}(q, \omega)=0$, as obtained from Eq. (A7) with $\beta=(3 / 5)^{1 / 2} k_{F}$ and $\epsilon_{b}=1$. (b) Logarithmic-scale representation of some of the curves in (a) compared to the light line $(\omega=q c)$ and the transverse bulk plasmon dispersion relation $\left(\omega=\left[\omega_{p}^{2}+q^{2} c^{2}\right]^{1 / 2}\right)$. The latter, which is obtained by inserting Eq. (A 8 ) into $q^{2}=k^{2} \epsilon_{\mathrm{tr}}$, gives rise to light emission in thin films irradiated by electrons (Ferrell, 1958; Vincent and Silcox, 1973).

specimens of different thicknesses (Mkhoyan et al., 2007).

One is often interested in opening the collection angle to increase the inelastic signal and to reduce sample damage. Nonlocal effects are then apparent, for example, through $e-h$ pair excitations, as shown in Fig. 8(a) for a free-electron gas of parameters corresponding to Al. For instance, this region is accessible when using $200 \mathrm{keV}$ electrons and $\varphi_{\text {out }}=10 \mathrm{mrad}$, a combination that results in the $q_{c}$ value indicated by the heavy arrow of Fig. 8(a). Under such circumstances, we need to include spatial dispersion in the dielectric function, which has different forms for longitudinal and transverse fields, $\epsilon_{\text {lon }}(q, \omega)$ and $\epsilon_{\mathrm{tr}}(q, \omega)$, respectively, as pointed out in Appendix A. The momentum-resolved loss probability is then found to be 


$$
\begin{aligned}
\frac{d \Gamma_{\text {bulk }}(\omega)}{d \mathbf{q}_{\perp}}= & \frac{e^{2} L}{\pi^{2} \hbar v^{2}} \operatorname{Im}\left\{\frac{1}{q^{2}}\left(\frac{1}{\epsilon_{\mathrm{tr}}(q, \omega)}-\frac{1}{\epsilon_{\mathrm{lon}}(q, \omega)}\right)\right. \\
& \left.+\left(\frac{v^{2}}{c^{2}}-\frac{1}{\epsilon_{\mathrm{tr}}(q, \omega)}\right) \frac{1}{q^{2}-k^{2} \epsilon_{\mathrm{tr}}(q, \omega)}\right\},
\end{aligned}
$$

where $\mathbf{q}_{\perp}$ is the $2 \mathrm{D}$ wave-vector transfer perpendicular to the electron trajectory, and the total wave-vector transfer satisfies $q^{2}=q_{\perp}^{2}+\omega^{2} / v^{2}$ by virtue of Eq. (3).

The momentum dependence of the transverse permittivity can be safely neglected in this discussion since it constitutes a higher-order correction in $v_{F} / c$ to the response, where $v_{F}$ is the Fermi velocity (e.g., $v_{F}=1.39$ $\times 10^{8} \mathrm{~cm} \mathrm{~s}^{-1}$ in gold). In addition, the Lindhard formula (Lindhard, 1954) for the response of a free-electron gas (see explicit expression in Appendix A.1) provides a fair description of the longitudinal dielectric function in good metals such as Al, although local field corrections can also be relevant (Vast et al., 2002).

In this context, the response of gold has increasing technological importance, but detailed experimental analysis of the momentum-dependent optical constants is still missing. The local dielectric function determined by Johnson and Christy (1972) from ellipsometry measurements is routinely employed in plasmonic studies, and it can be well described by the Drude-like formula of Eq. (A8) in the visible and NIR part of the spectrum (see Table II). Recently García de Abajo (2008) proposed a nonlocal $\epsilon_{\mathrm{lon}}$ for gold constructed as

$$
\epsilon_{\mathrm{lon}}(q, \omega)=\epsilon^{\operatorname{expt}}(\omega)-\epsilon^{\mathrm{D}}(\omega)+\epsilon^{\mathrm{M}}(q, \omega),
$$

where $\epsilon^{\mathrm{D}}$ and $\epsilon^{\mathrm{M}}$ are the Drude and Mermin dielectric functions given by Eqs. (A1) and (A5), respectively, and corresponding to the values of $\omega_{p}$ and $\eta$ listed in Table II, whereas $\epsilon^{\text {expt }}$ is the measured local permittivity. ${ }^{10}$ Further experimental effort is still required to compare with this model.

The spectrum in bulk metals is dominated by plasmon losses, first observed by Lang (1948) and Ruthermann (1948) and later identified as collective oscillations of the conduction-electron gas by Pines and Bohm (1952), giving rise to Poissonian multiple-loss distributioms in electrons traversing metallic foils (Marton et al., 1962). These modes are signaled by the vanishing of $\epsilon_{\text {lon }}$, as deduced from the NR limit of Eq. (20),

$$
\frac{d \Gamma_{\mathrm{bulk}}^{\mathrm{NR}}(\omega)}{d \mathbf{q}_{\perp}}=\frac{e^{2} L}{\pi^{2} \hbar v^{2} q^{2}} \operatorname{Im}\left\{-\frac{1}{\epsilon_{\mathrm{lon}}(q, \omega)}\right\} .
$$

The dispersion relation of bulk plasmons has been studied in the past using EELS (Watanabe, 1956; Batson et al., 1976; Chen et al., 1976, 1980; Raether, 1980) and is shown in Fig. 8(a) for $\mathrm{Al}$, as measured by Batson and Silcox (1983) (symbols). When broad collection angles are considered, one can reliably approximate $\epsilon_{\text {lon }}(q, \omega)$

\footnotetext{
${ }^{10}$ The measured local response $\epsilon^{\text {expt }}$ is corrected by subtracting the local response of conduction electrons $\left(\epsilon^{\mathrm{D}}\right)$ and by replacing it with the nonlocal response of those electrons in the Mermin model $\left(\epsilon^{\mathrm{M}}\right)$.
}

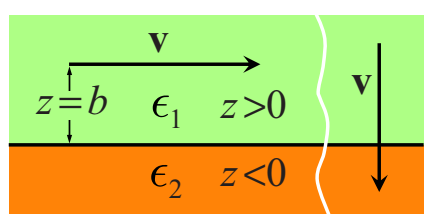

FIG. 9. (Color online) Planar interface separating two media of dielectric functions $\epsilon_{1}$ and $\epsilon_{2}$, respectively. An electron is shown moving parallel to the boundary inside medium 1 (left), with impact parameter $b$ relative to the interface. Another electron is represented crossing the interface at normal incidence (right).

by a plasmon-pole analytical expression (Ritchie, 1957), reproduced in Eq. (A7). The plasmon dispersion relation derived from this formula provides a reasonable interpolation between the measured plasmon and the Bethe ridge $\omega=\hbar q^{2} / 2 m_{e}$, shown in Fig. 8(a) as a solid curve.

Spatially resolved bulk-plasmon spectroscopy has been used to identify the presence of metals in a specimen [e.g., $\mathrm{Na}$ in either $\mathrm{Na}_{2} \mathrm{O}$ or silicate glasses (Jiang et al., 2008)] and to demonstrate the effect of quantum confinement in carbon nanostructures by tracing local variations in the plasmon energy (Stolojan et al., 2006), which are related to changes in the conduction-electron density through Eq. (A2).

Finally, magnetic circular dichroism has been measured in the response of iron at $\mathrm{x}$-ray energies (Schattschneider et al., 2006), suggesting that further work could eventually explore optical activity at visible and NIR frequencies via EELS, for instance, in chiral (Rogacheva et al., 2006) and left-handed (Boltasseva and Shalaev, 2008) metamaterials.

\section{Planar surfaces}

Surfaces and interfaces can host trapped modes that modify the local band structure and the optical response with respect to bulk materials. For instance, we find not only electronic surface states confined by directional gaps in noble-metal interfaces (Smith, 1985) but also specific collective modes of conduction electrons (e.g., surface plasmons). As shown later, these features can be characterized by EELS performed in STEMs with a high degree of spatial resolution.

A particularly instructive situation is presented when the beam is directed parallel to a planar interface separating two different media (Fig. 9, left). This configuration has been extensively studied both experimentally (Powell, 1968; Lecante et al., 1977; Walls and Howie, 1989; Moreau et al., 1997) and theoretically (Lucas and Šunjić, 1971; Echenique and Pendry, 1975; GarcíaMolina, Gras-Martí, Howie, and Ritchie, 1985). The analysis of this geometry in the nonretarded limit can be readily made from the knowledge of the screened interaction $W\left(\mathbf{r}, \mathbf{r}^{\prime}, \omega\right)$, defined as the potential created at point $\mathbf{r}$ by a unit charge oscillating with frequency $\omega$ and placed at $\mathbf{r}^{\prime}$. Expressions of $W$ for simple geometries, including that of Fig. 9, are given in Appendix B. A closed-form expression for the loss probability of the 
electron following the parallel trajectory of Fig. 9 (left) is then obtained from Eqs. (10) and (B3). One finds

$$
\begin{aligned}
\Gamma_{\text {planar }}^{\mathrm{NR}}(\omega)= & \frac{2 e^{2} L}{\pi \hbar v^{2}}\left\{\ln \left(\frac{q_{c} v}{\omega}\right) \operatorname{Im}\left\{-\frac{1}{\epsilon_{1}}\right\}+K_{0}\left(\frac{2 \omega b}{v}\right)\right. \\
& \left.\times\left[\operatorname{Im}\left\{-\frac{2}{\epsilon_{1}+\epsilon_{2}}\right\}-\operatorname{Im}\left\{-\frac{1}{\epsilon_{1}}\right\}\right]\right\},
\end{aligned}
$$

where $L$ is the length of the trajectory. The impact parameter $b$ and the dielectric functions $\epsilon_{1}$ and $\epsilon_{2}$ are defined in Fig. 9. The first term inside the curly brackets is the bulk loss probability of Eq. (19). The square brackets in Eq. (23) contain the effect of the interface, which dies off exponentially with $b$ as $K_{0}(\theta) \approx \exp (-\theta) \sqrt{\pi / 2 \theta}$ for large $\theta=2 \omega b / v$ (Abramowitz and Stegun, 1972). The first interface term describes the excitation of intrinsic boundary modes, signaled by the condition $\epsilon_{1}+\epsilon_{2}=0$. It was first derived by Echenique and Pendry (1975), who obtained

$$
\Gamma_{\text {planar }}^{\mathrm{NR}}(\omega)=\frac{4 e^{2} L}{\pi \hbar v^{2}} K_{0}\left(\frac{2 \omega b}{v}\right) \operatorname{Im}\left\{-\frac{1}{1+\epsilon}\right\}
$$

for an electron moving in vacuum near a medium of dielectric function $\epsilon$. The second interface term in Eq. (23) accounts for a reduction of bulk losses (i.e., the transfer of oscillator strength from volume to surface modes). This is the so-called "begrenzungseffekt."

Equation (23) has been successfully applied to explain surface losses suffered by electrons passing near $\mathrm{MgO}$ cubes (Cowley, 1982a, 1982b; Marks, 1982; Wang and Cowley, 1988; Walls and Howie, 1989) in the so-called aloof configuration (see below) and also to EELS in a $\mathrm{Si} / \mathrm{SiO}_{2}$ interface and in GaAs surfaces (Howie and Milne, 1985).

Inclusion of retardation effects is critical to account for CR losses and to correctly assess the weight of interface effects, as demonstrated by Moreau et al. (1997) for the $\mathrm{Si} / \mathrm{SiO}_{2}$ interface and later by Couillard et al. (2007) for multilayer structures containing HfO. These studies have been recently extended by Couillard et al. (2008) and Yurtsever et al. (2008), who have measured EELS in slabs and layered stacks formed by $\mathrm{Si}$ and $\mathrm{SiO}_{2}$ using electron beams parallel to the interfaces and have demonstrated the important role of retardation corrections for a quantitative comparison with theory.

The retarded counterpart of Eq. (23) has been reported by several researchers (Otto, 1967; GarcíaMolina, Gras-Martí, Howie, and Ritchie, 1985). A particularly simple derivation consists in expanding the integrand of Eq. (2) into $p$ (TM) and $s$ (TE) electromagnetic plane-wave components, which are later reflected at the interface to act back on the electron (Forstmann et al., 1991). One finds

$$
\Gamma_{\text {EELS }}(\omega)=\Gamma_{\text {bulk }}(\omega)+\Gamma_{\text {ref }}(\omega),
$$

where $\Gamma_{\text {bulk }}$ is the bulk loss inside the medium 1 in which the electron is moving, given by Eq. (18), and

$$
\begin{aligned}
\Gamma_{\text {ref }}(\omega)= & \frac{2 e^{2} L}{\pi \hbar v^{2}} \int_{0}^{\infty} \frac{d q_{y}}{q_{\|}^{2}} \operatorname{Re}\left\{q_{z 1} e^{2 i q_{z 1} b}\right. \\
& \left.\times\left[\left(\frac{q_{y} v}{q_{z 1} c}\right)^{2} r_{s}-\frac{1}{\epsilon_{1}} r_{p}\right]\right\}
\end{aligned}
$$

is the loss due to reflection of the electron field at the interface. Here $q_{\|}=\sqrt{\omega^{2} / v^{2}+q_{y}^{2}}, q_{z j}=\sqrt{k^{2} \epsilon_{j}-q_{\|}^{2}}$, and

$$
r_{p}=\frac{\epsilon_{2} q_{z 1}-\epsilon_{1} q_{z 2}}{\epsilon_{2} q_{z 1}+\epsilon_{1} q_{z 2}}
$$

and

$$
r_{s}=\frac{q_{z 1}-q_{z 2}}{q_{z 1}+q_{z 2}}
$$

are Fresnel reflection coefficients for $p$ and $s$ waves, respectively. In the nonretarded limit, one has $r_{s}=0$ and $r_{p}=\left(\epsilon_{2}-\epsilon_{1}\right) /\left(\epsilon_{2}+\epsilon_{1}\right)$, from which Eq. (23) is easily recovered. The advantage of this approach, based on optical reflection coefficients, is that it can handle more complicated surfaces, such as the periodically corrugated boundary of a confined photonic crystal (Pendry and Martín-Moreno, 1994; García de Abajo and Blanco, 2003). For large impact parameters, Eq. (25) provides a retardation correction to Eq. (24), consisting in substituting $2 \omega b / v \gamma$ for $2 \omega b / v$ in the argument of the $K_{0}$ Bessel function; the apparent impact parameter at large velocity is contracted by $b / \gamma$ (Mkhoyan et al., 2007).

\section{Excitation of surface plasmons and surface plasmon polaritons}

Surface modes, signaled by the condition $\epsilon_{1}+\epsilon_{2}=0$, are characteristic of the interface between a dielectric and a metal. These excitations were first identified and understood by Ritchie (1957) to explain anomalous valence losses that were previously observed at positions differing from bulk plasmons in the spectra of fast electrons transmitted through thin foils (see, for instance, feature D in Fig. 2 of Watanabe, 1956). Ritchie's description in terms of a classical hydrodynamic plasma to represent the conduction electron band led Stern and Ferrell (1960) to call these excitations surface plasmons, which were later confirmed in electron energy-loss experiments (Powell and Swan, 1959) (see Fig. 10). Bulk and surface modes were observed as $\hbar \omega_{p} \approx 15 \mathrm{eV}$ and $\hbar \omega_{s} \approx 10.6 \mathrm{eV}$ energy losses in electrons reflected from an $\mathrm{Al}$ surface. [Incidently, the spectrum of Fig. 10 contains multiple plasmon losses (i.e., two or more plasmons being excited by the same electron). The coherent aspects of double plasmon excitation have received some attention in the past (Schattschneider et al., 1987).]

Aluminum is a prototypical example of a nearly-freeelectron gas for which the dielectric function is well described by the Drude formula of Eq. (A1). The SP of the bare surface, satisfying the condition $\epsilon+1=0$, then has a frequency $\omega_{s}=\omega_{p} / \sqrt{2}$, in good agreement with experimental observations. 


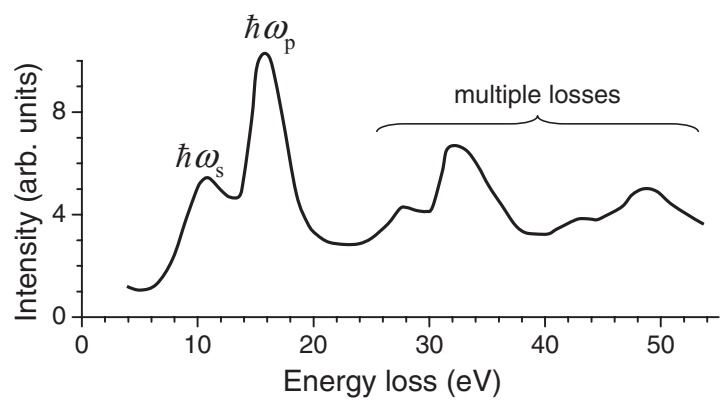

FIG. 10. First observation of surface plasmons. They were detected in the energy-loss distribution of $2020 \mathrm{eV}$ electrons specularly reflected on an $\mathrm{Al}$ surface under $45^{\circ}$ incidence. Adapted from Powell and Swan, 1959.

Surface plasmons were also observed for other materials in subsequent reflection experiments performed on melted metals (Powell, 1968); even the evolution of these modes through a solid-liquid phase transition was reported (Powell, 1965). Other pioneering electron transmission experiments managed to demonstrate the observation of SPs as a characterization technique (Raether, 1967) and to determine the dependence of SP energies on the thickness of thin films (Boersch, Geiger, Imbusch, and Niedrig, 1966). Evidence of surface and bulk plasmon excitation has also been observed in the spectra of photoelectrons and Auger electrons emitted from metals (van Attekum and Trooster, 1978; Osterwalder et al., 1990; Simonsen et al., 1997).

Like the bulk plasmons of Fig. 8(a), SPs are affected by nonlocal effects and exhibit significant dispersion, which was reported in early EELS studies (Krane and Raether, 1976). This has stimulated a rich literature intended to achieve a reasonable first-principles description of the dynamical response of crystal surfaces [see Pitarke et al. (2007), and references therein]. Nonlocal effects involve comparatively short-range interactions extending a distance $\sim 1 / q$ for typical wave-vector transfers $q$ in the $\AA^{-1}$ domain [see Fig. 8(a)]. However, we are mainly concerned here with longer-range optical excita- tions (e.g., SPPs), in which space dispersion can be generally overlooked, except when sharp metallic structures or narrow gaps between metals are involved (García de Abajo, 2008), as shown in Sec. V.E, for electrons passing near metal-sphere dimers. Nonlocal effects can also be significant at small separations $\leqslant 1 \mathrm{~nm}$ between the passing electron and metal surfaces (Zabala and Echenique, 1990).

An interesting scenario is presented by the aloof configuration, in which the electron trajectory does not even intersect the sample. This leads to distant interaction, capable of providing surface-specific information, free of close-encounter events between the fast probe and the target atoms. The exponential decay of surface losses with $2 \omega b / v \gamma$ indicates that low-energy transfers are favored in the aloof configuration. This has the additional benefit of minimizing sample damage.

The aloof configuration was pioneered by Lecante $e t$ al. (1977), who forced the electrons to describe parabolic trajectories, deflected from the metal surface by a bias potential. Subsequent studies managed to aim a STEM aloof beam parallel to the planar surfaces of $\mathrm{MgO}$ cubes (Cowley, 1982a, 1982b; Marks, 1982). Loss spectra were later recorded after transmission through perforated metallic channels (Warmack et al., 1984). The aloof configuration has been recently reconsidered and compared with near-field optical microscopy (Cohen et al., 1998, 1999, 2003; Echenique et al., 1999; Itskovsky et al., 2008).

Retardation adds another source of plasmon dispersion. Since SP modes are resonances in the surface response, they must involve a divergence in the reflectivity for incident evanescent waves with the right values of $\left(q_{\|}, \omega\right)$. More precisely, we obtain the plasmon dispersion relation from the vanishing of the denominator of $r_{p}$ [Eq. (26)], leading to

$$
q_{\|}^{\mathrm{SP}}=k \sqrt{\frac{\epsilon_{1} \epsilon_{2}}{\epsilon_{1}+\epsilon_{2}}}
$$

under the condition that $\operatorname{Re}\left\{\epsilon_{1}\right\}$ and $\operatorname{Re}\left\{\epsilon_{2}\right\}$ have opposite signs. The dispersion relation of Eq. (27) is repre-

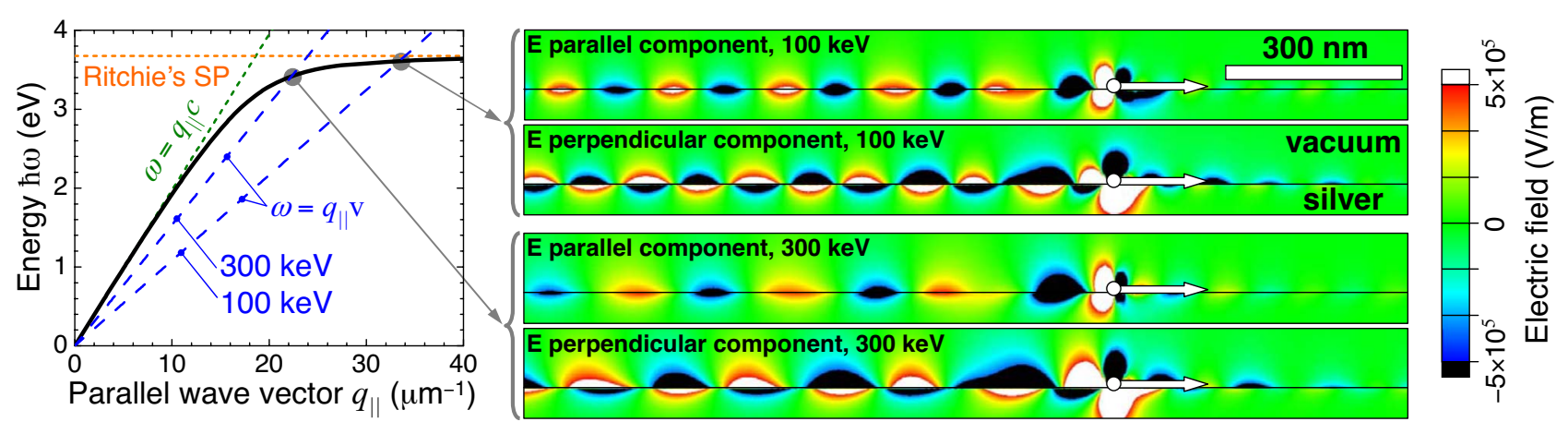

FIG. 11. (Color online) Surface plasmon dispersion relation in Ag (left) and excitation of SPPs by swift electrons (right). The SPPs saturate at Ritchie's nonretarded frequency for large parallel wave vector and follow the light line $\omega=q_{\|} c$ in the low-energy limit. An electron moving with velocity $\mathbf{v}$ parallel to the surface preferentially excites plasmons of frequency and wave vector related by $\omega \lesssim q_{\|} v$. This condition is indicated by solid dots in the dispersion relation for the two electron energies considered on the right. Faster electrons generate plasmons of lower $q_{\|}$and longer wavelength. The impact parameter is $10 \mathrm{~nm}$ in the field plots. The dielectric function of $\mathrm{Ag}$ is taken from Palik (1985). 
sented in Fig. 11 (left) for a silver-vacuum interface. When the retarded nature of SPs becomes important, they are usually referred to as SPPs because they acquire a polaritonic character reflecting a mixture of collective electron motion and propagating electromagnetic fields. This tends to happen at low wave vectors (i.e., at long wavelengths according to Fig. 11), for which SPPs increasingly approach the light line $\left(\omega=q_{\|} c\right)$.

It is important to stress that this polaritonic regime occurs for momentum transfers that are well below the region exhibiting nonlocal effects [compare the horizontal scales in Figs. 8(a) and 11]. This is shown in Fig. 8(b), where the hybridization of transverse bulk plasmons with light takes place at values of $q$ that are two orders of magnitude smaller than the Fermi wave vector $k_{F}$. More details on SPPs, including a comprehensive literature survey, can be found in Raether (1988).

The electric and magnetic fields associated with a SPP plane wave moving along $x$ have similar strength in Gaussian units. For instance, in the vacuum region outside a semi-infinite metal of permittivity $\epsilon$, we have

$$
\begin{aligned}
& \mathbf{E}^{\mathrm{SP}}(\mathbf{r}, \omega)=\left[\left(\hat{\mathbf{x}} q_{z}^{\mathrm{SP}}-\hat{\mathbf{z}} q_{\|}^{\mathrm{SP}}\right) / k\right] \exp \left[i\left(q_{\|}^{\mathrm{SP}} x+q_{z}^{\mathrm{SP}} z\right)\right], \\
& \mathbf{H}^{\mathrm{SP}}(\mathbf{r}, \omega)=\hat{\mathbf{y}} \exp \left[i\left(q_{\|}^{\mathrm{SP}} x+q_{z}^{\mathrm{SP}} z\right)\right],
\end{aligned}
$$

where $q_{z}^{\mathrm{SP}}=\sqrt{k^{2}-\left(q_{\|}^{\mathrm{SP}}\right)^{2}}=-k / \sqrt{\epsilon+1}$. In particular, the electric field satisfies $\boldsymbol{\nabla} \cdot \mathbf{E}^{\mathrm{SP}}=0$, and therefore it is transverse.$^{11}$ Close to the plasmon cutoff, the SPP momentum becomes increasingly large. This marks the transition toward the nonretarded regime, in which magnetic and electric fields are decoupled, so that we can approximate $\boldsymbol{\nabla} \times \mathbf{E}^{\mathrm{SP}}=\mathbf{0}$ and define a potential $\phi \propto \exp \left[(i x-z) q_{\|}^{\mathrm{SP}}\right]$ to write $\mathbf{E}^{\mathrm{SP}}=-\boldsymbol{\nabla} \phi$. The electric field appears to be longitudinal, and the plasmons are then known as SPs rather than SPPs, signaled by the condition $\epsilon=-1$.

An aloof electron like that of Fig. 9 (left) couples to SPPs of momentum component along the velocity vector given by $\hbar \omega / v$, according to Eq. (3). Consequently, the electron excites only parallel momenta above that value. This condition is represented in Fig. 11 (left) for two different electron energies. The corresponding electric fields are shown in the same figure (right) within a plane that contains the velocity vector and the surface normal. The electron is passing at a distance of $10 \mathrm{~nm}$ above the surface. We observe several characteristic features in these plots: (1) the field shows a wake pattern dominated by oscillations of wavelength $\approx 2 \pi / q_{\|}$, with $q_{\|}=\omega / v$ determined by the intersection points of Fig. 11 (left); (2) the electron that moves faster excites plasmons of lower energy, thus giving rise to oscillations of longer spatial period; (3) at variance with the continuity of the parallel

\footnotetext{
${ }^{11}$ Although the main $\mathbf{E}^{\mathrm{SP}}$ component of long-wavelength SPPs is along the surface normal, there is also a finite electric field along the propagation direction, but the normal components of both the wave vector and $\mathbf{E}^{\mathrm{SP}}$ have a $\pi / 2$ phase difference relative to the parallel components, leading to $\boldsymbol{\nabla} \cdot \mathbf{E}^{\mathrm{SP}}$ $=0$.
}

electric field, the normal component changes sign across the surface in order to preserve the continuity of the normal displacement because the metal permittivity is negative; (4) the normal field component takes large values compared to the parallel one, and this effect is more pronounced for faster electrons, which involve lower $\omega$ 's. These observations are consistent with the qualitative features of the above equations.

In a different direction, the early 1990s witnessed remarkable experiments of energy losses in coincidence with SEE, initially collected in amorphous carbon samples (Pijper and Kruit, 1991; Müllejans and Bleloch, 1992). Angle-resolved measurements were also carried out in this context to conclude that the SEE yield is larger in more localized excitations, involving largermomentum transfers (Drucker and Scheinfein, 1993). Comparative studies for amorphous carbon and silicon suggested that bulk plasmons do not play a central role in SEE (Drucker et al., 1993; Scheinfein et al., 1993), possibly due to the noted begrenzungseffekt, which limits the strength of volume plasmons in favor of SPs within the region accessible to the escape depth of SEs. Finally, coincidence experiments were conducted using the aloof beam geometry in diamond and $\mathrm{MgO}$ to compare EELS and SEE rates and to provide direct measurements of the probability that a surface excitation gives rise to a SE, which was found to be below $\sim 5 \%$ for low-energy losses (Müllejans et al., 1993).

\section{Guided modes in thin films}

The SPPs on both sides of a thin film interact to produce two hybridized modes. One of them can travel longer distances along the film due to exclusion of the electric field from the metal (Sarid, 1981). This mode is currently being applied to propagate electromagnetic signals in plasmonic devices (Barnes et al., 2003; Berini et al., 2007). The dispersion relation of coupled SPPs in thin films was obtained from the EELS signal of transmitted electrons over 30 years ago by a series of outstanding experiments conducted by Silcox and coworkers in Al (Vincent and Silcox, 1973; Pettit et al., 1975) and Si (Chen et al., 1975) films. An example of these studies is given in Fig. 12 for a partially oxidized thin $\mathrm{Al}$ film. Both plasmon branches follow the light line at low energies, and they converge to the nonretarded limit of the $\mathrm{Al} / \mathrm{Al}_{2} \mathrm{O}_{3}$ interface for large momentum transfer. This limit corresponds to $\epsilon_{\mathrm{Al}}+4=0$ or, equivalently, $\hbar \omega_{s} \approx 6.7 \mathrm{eV}$ when Eq. (A1) is used to model $\mathrm{Al}$ since the permittivity of $\mathrm{Al}_{2} \mathrm{O}_{3}$ in this energy window is $\approx 4$. Large $q_{\|}$'s involve fast plasmon oscillations along the film, so that the alternating induced charges weaken the interaction between the two film sides and we recover the limit of the single interface separating two semiinfinite media. For small $q_{\|}$, the interaction between plasmons in both sides of the film gives rise to two SPP branches.

Guided modes in graphite (Chen and Silcox, 1975a) and aluminum-oxide (Chen and Silcox, 1975b) films were also characterized using the same technique. In this 


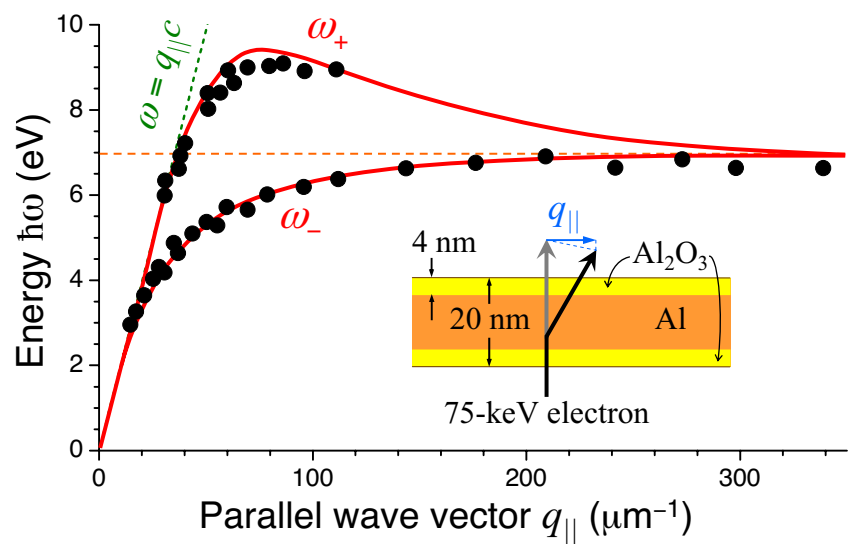

FIG. 12. (Color online) Experimental determination of the SPP dispersion relation in a thin film. Symbols: Measured data obtained from energy and angle distributions of fast electrons traversing a partially oxidized Al thin film. Continuous curves: SPP branches calculated from optical data. Aadapted from Pettit et al., 1975.

case, the film behaved as a dielectric, and the electron coupled to optical Fabry-Perot resonances set up by successive total internal reflections at the film boundaries. The experimental results were in good agreement with theory (Chen and Silcox, 1979).

In a more recent development, a complex multilayer structure containing $\mathrm{HfO}$, which is of interest in microelectronics, has been characterized by Couillard et al. (2007). The agreement with theory is excellent after retardation effects are incorporated to correctly account for interface plasmons and Cherenkov guided modes. A similar study has been recently presented by Yurtsever et al. (2008) in slabs and stacks of $\mathrm{Si}$ and $\mathrm{SiO}_{2}$.

Additionally, a radiative plasmon branch inside the light cone, giving rise to losses near $\omega_{p}$, was predicted by Ferrell (1958) for thin metal films, and the resulting light emission was detected later by Steinmann (1960) in Ag. We discuss this issue in more detail in Sec. IV.F.1.

\section{Curved geometries}

The ability of TEMs to probe local response properties has been extensively exploited to study nonplanar sample geometries. Particular attention has been paid to spherical and cylindrical shapes, carbon nanotubes, composites, and other more complicated structures. The intense valence losses can provide spectral images, the interpretation of which is not as direct as when core losses are employed instead. Therefore, we need to strongly rely on theory, which is actually in a very advanced state, capable of dealing with virtually any system. For example, small particles such as fullerenes or carbon nanotubes are reliably described by atomistic models, although first-principles theory is also possible (Marinopoulos et al., 2003). For larger structures (e.g., nanoparticles) we have a suite of methods based on classical dielectric theory, which gives good agreement with experiment in metallic objects of dimensions above

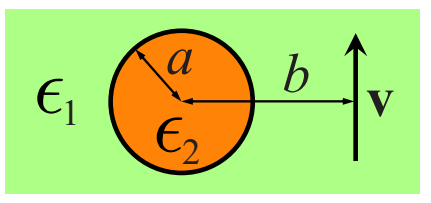

FIG. 13. (Color online) Notation used for homogeneous spheres and cylinders of radius $a$, with an electron passing at a distance $b$ from the center.

$\sim 10 \mathrm{~nm}$. In this section, we explore some of these structures possessing spherical or cylindrical geometry.

\section{Cylinders}

Early work on cylindrical nanocavities relied on an electron beam to drill holes in $\mathrm{AlF}_{3}$ (Macaulay et al., 1989), which were investigated by EELS (Scheinfein et al., 1985). The synthesis of such types of holes has considerably improved over the last few years in two different directions. (1) The bottom-up approach: chemical methods have been developed that are capable of spontaneously forming arrays of self-organized nanoholes in materials such as alumina (Masuda and Fukuda, 1995); arrays of cylinders are also formed in eutectics (Pawlak et al., 2008). (2) The top-down approach: advanced electron-beam and focused-ion-beam lithographies have been extensively employed to produce hole arrays for nanophotonics studies (Genet and Ebbesen, 2007). These and similar geometries have been investigated through TEM spectroscopic analysis (García de Abajo et al., 2003; Degiron et al., 2004).

Likewise, self-standing Si (Reed et al., 1999) and Ge (Hanrath and Korgel, 2004) nanowires have been studied to show clear evidence of the begrenzungseffekt in the former and confinement effects in the position of the bulk plasmons in the latter. Interface losses in $\mathrm{Bi}$ wires embedded in an alumina matrix have also been observed (Sander et al., 2001) and theoretically explained (García de Abajo and Howie, 2002).

The axial symmetry of a cylinder enables us to classify its modes according to their azimuthal dependence $\exp (i m \varphi)$, whereas a separation into $\exp \left(i q_{z} z\right)$ components of wave vector $q_{z}$ is appropriate for the variation along the direction of translational invariance, chosen along $\hat{\mathbf{z}}$. This decomposition is employed in Appendix B.2 to write the nonretarded screened interaction from which the cylinder modes are derived using the condition $\Delta_{m}=0$ [see Eq. (B4)]. A relatively simple expression can be obtained for these modes including retardation (Ashley and Emerson, 1974),

$$
\begin{aligned}
x_{1}^{2} x_{2}^{2} & {\left[\epsilon_{2} x_{1} I_{m}^{\prime}\left(x_{2}\right) / I_{m}\left(x_{2}\right)-\epsilon_{1} x_{2} K_{m}^{\prime}\left(x_{1}\right) / K_{m}\left(x_{1}\right)\right] } \\
& \times\left[x_{1} I_{m}^{\prime}\left(x_{2}\right) / I_{m}\left(x_{2}\right)-x_{2} K_{m}^{\prime}\left(x_{1}\right) / K_{m}\left(x_{1}\right)\right] \\
= & m^{2}\left(\epsilon_{1}-\epsilon_{2}\right)^{2}\left(k q_{z} a^{2}\right)^{2},
\end{aligned}
$$

where the notation of Fig. 13 has been adopted, the subscripts 1 and 2 refer to the media outside and inside the cylinder, respectively, and $x_{j}=a \sqrt{q_{z}^{2}-\epsilon_{\mathrm{j}} k^{2}}$. 
The interaction of the electron with a cylinder has been described using both nonretarded (Zabala et al., 1989; Tu et al., 2006) and fully retarded (Zabala et al., 1989; Walsh, 1991) dielectric theories. One can easily derive the loss probability using the screened interaction $W$ in the nonretarded limit [see Eq. (B4)]. For electrons moving parallel to a cylinder, one needs to insert $W$ into Eq. (10). The momentum transfer along the cylinder axis is fully determined by the condition $q_{z}=\omega / v$, and one obtains the loss probability

$$
\Gamma_{\mathrm{cyl}}^{\mathrm{NR}}\left(\mathbf{R}_{0}, \omega\right)=\frac{e^{2} L}{2 \pi^{2} \hbar v^{2}} \sum_{m=-\infty}^{\infty} \operatorname{Im}\left\{-W_{m}\left(b, b, q_{z}, \omega\right)\right\},
$$

where $b$ is the distance from the electron beam to the cylinder axis and different $m$ 's contribute separately. Retardation effects, however, are tedious to deal with analytically (Zabala et al., 1989; Walsh, 1991) as compared to the planar interface. For instance, a beam moving along the center of the cylinder, which only couples to $m=0$ modes due to the symmetry of the electroncylinder combined system, experiences a loss probability

$$
\begin{aligned}
\Gamma_{\text {ref,cyl }}(\omega)= & \frac{2 e^{2} L}{\pi \hbar v^{2}} \operatorname{Im}\left\{\left(\frac{v^{2}}{c^{2}}-\frac{1}{\epsilon_{2}}\right)\right. \\
& \left.\times \frac{\epsilon_{2} x_{1} K_{0}\left(x_{1}\right) K_{1}\left(x_{2}\right)-\epsilon_{1} x_{2} K_{1}\left(x_{1}\right) K_{0}\left(x_{2}\right)}{\epsilon_{2} x_{1} K_{0}\left(x_{1}\right) I_{1}\left(x_{2}\right)+\epsilon_{1} x_{2} K_{1}\left(x_{1}\right) I_{0}\left(x_{2}\right)}\right\},
\end{aligned}
$$

where we can immediately see the presence of the $m=0$ mode predicted by Eq. (28) when we arrange the denominator of the last fraction as $K_{0}\left(x_{1}\right) I_{0}\left(x_{2}\right)\left[\epsilon_{2} x_{1} I_{0}^{\prime}\left(x_{2}\right) / I_{0}\left(x_{2}\right)-\epsilon_{1} x_{2} K_{0}^{\prime}\left(x_{1}\right) / K_{0}\left(x_{1}\right)\right], \quad$ with $q_{z}=\omega / v$, and therefore, $x_{j}=(\omega a / v) \sqrt{1-\epsilon_{j} v^{2} / c^{2}}$.

\section{Spheres}

The interaction of electrons with spheres has attracted considerable attention since the publication of pioneering experimental loss spectra in alkali-metal halide (Creuzburg, 1966) and Al (Fujimoto et al., 1967) particles, followed by their subsequent theoretical interpretation (Crowell and Ritchie, 1968; Fujimoto and Komaki, 1968). Further EELS measurements corroborated these results and focused on the role of plasmon dispersion in small spheres (Batson, 1980; Ouyang et al., 1992) and in the effect of the interaction between neighboring particles (Batson, 1982b, 1985; Ugarte et al., 1992). More recently, uv surface exciton polaritons have been observed in gold nanospheres (Chu et al., 2008) and whispering gallery modes measured in silica beads (Hyun et al., 2008) using EELS.

A small metallic sphere embedded in a dielectric host exhibits plasmon modes at frequencies dictated by the condition (Kittle, 1987)

$$
(l+1) \epsilon_{1}+l \epsilon_{2}=0,
$$

where $l$ labels the multipolar order of the excitation and $\epsilon_{1}$ and $\epsilon_{2}$ are the permittivities of the host and the metal, respectively (see Fig. 13). In particular, for an Al sphere in which $\epsilon_{2}$ is described by the Drude dielectric function of Eq. (A1), the frequency of the nonretarded plasmon oscillations becomes

$$
\omega=\omega_{p} \sqrt{\frac{l}{(l+1) \epsilon_{1}+l}} .
$$

For example, the dipole $(l=1)$ of an $\mathrm{Al}$ sphere in vacuum has energy $\hbar \omega_{p} / \sqrt{3} \approx 8.7 \mathrm{eV}$. For very large $l$, the surface charge oscillations are so dense that they cannot resolve the particle curvature, and therefore the mode frequency evolves to the plasmon of the planar interface, $\omega_{s}=\omega / \sqrt{\epsilon_{1}+1}$.

Crowell and Ritchie (1968) and Fujimoto and Komaki (1968) established the basis for the theoretical understanding of fast-electron interaction with spherical particles. They described a metallic sphere using the hydrodynamical model of Appendix A.2 and derived analytical expressions for the loss probability and photon emission rate of a broad beam, neglecting retardation. Their results showed spectral features at frequencies roughly described by Eq. (30).

This theoretical development was followed by studies of voids in metals (Ashley and Ferrell, 1976) and later by the derivation of an analytical formula for the loss probability of a STEM electron passing outside a sphere (Ferrell and Echenique, 1985; Ferrell et al., 1987). Namely,

$$
\begin{aligned}
\Gamma_{\mathrm{sph}}^{\mathrm{NR}}(\omega)= & \frac{4 e^{2}}{\pi \hbar v^{2}} \sum_{l=1}^{\infty} \sum_{m=-l}^{l} \frac{(\omega / v)^{2 l}}{(l+m) !(l-m) !} K_{m}^{2}\left(\frac{\omega b}{v}\right) \\
& \times \operatorname{Im}\left\{\alpha_{l}^{\mathrm{NR}}(\omega)\right\}, \quad k b \ll 1, \quad v / c \ll 1,
\end{aligned}
$$

where

$$
\alpha_{l}^{\mathrm{NR}}(\omega)=a^{2 l+1} \frac{l \epsilon(\omega)-l}{l \epsilon(\omega)+l+1}
$$

is the nonretarded multipolar polarizability of a sphere of radius $a$ in vacuum and $b$ is the distance from the trajectory to the sphere center, as shown in Fig. 13 (we take $\epsilon_{1}=1$ and $\epsilon_{2}=\epsilon$ ). Equation (31) can be derived upon insertion of the nonretarded screened interaction near a sphere [Eq. (B6)] into Eq. (10). This formula successfully explained previous measurements for aloof electrons (Batson, 1982a) and was soon applied to explain EELS of spheres made of tin (Achèche et al., 1986), silicon (Ugarte et al., 1992), silver (Ouyang et al., 1992), and aluminum (Stöckli et al., 1997), as well as corrugated metal surfaces (Mamola et al., 1987) and diamond particles (Fehlhaber and Bursill, 1999, 2000; Peng et al., 2001). The effect of coatings, which can be important due to oxidation (Ferrell et al., 1987; Ugarte et al., 1992), is described by Eq. (31) if we use the polarizability of a coated sphere instead of Eq. (32).

Optical modes in nanoparticles (e.g., plasmons in metallic spheres) are well known to be redshifted due to retardation when the particle size is not negligible in front of the wavelength (see Sec. IV.F.2): the interaction between distant regions of a particle is mediated by electromagnetic signals that propagate at the speed of light, but the signal simply takes longer to travel over larger 
distances as the particle dimensions increase, so that the period of the oscillation modes increases, thus producing the noted redshift. This effect was first described by Mie (1908) in analyzing light scattering by metallic spheres. Similar phenomena occur in particles made of polaritonic materials (Fuchs and Kliewer, 1968).

The extension of Eq. (31) to incorporate retardation also leads to an analytical formula (García de Abajo and Howie, 1998; García de Abajo, 1999c)

$$
\begin{aligned}
\Gamma_{\mathrm{sph}}(\omega)= & \frac{e^{2}}{c \hbar \omega} \sum_{l=1}^{\infty} \sum_{m=-l}^{l} K_{m}^{2}\left(\frac{\omega b}{v \gamma}\right) \\
& \times\left[C_{l m}^{M} \operatorname{Im}\left\{t_{l}^{M}\right\}+C_{l m}^{E} \operatorname{Im}\left\{t_{l}^{E}\right\}\right],
\end{aligned}
$$

where the Lorentz factor $\gamma=1 / \sqrt{1-v^{2} / c^{2}}$ accounts for the contraction of the impact parameter $b$ as seen by the moving electron. Here $C_{l m}^{M}$ and $C_{l m}^{E}$ are coupling coefficients that depend exclusively on the $v / c$ ratio and $t_{l}^{M}$ and $t_{l}^{E}$ are magnetic and electric Mie scattering coefficients independent of beam energy and impact parameter. Analytical expressions for these coefficients are given in Appendix $\mathrm{C}$ for homogeneous spheres (see also Table III in Appendix C). Equation (33) results from a multipolar expansion of the electric field induced by the electron [Eq. (4)] after each multipole is scattered by the sphere to produce an outgoing wave acting back on the electron and giving rise to energy loss [see Eq. (8)].

It should be emphasized that Eq. (33) can be applied to any spherical object for external electron trajectories, provided $t_{l}^{M}$ and $t_{l}^{E}$ are known for that object. In particular, this has been recently done for left-handed spheres (Xu and Zhang, 2008) and spherical metamaterial cloaks (Xu et al., 2008).

Equation (33) predicts sizable redshifts even for relatively small $\mathrm{Al}$ spheres of radius $a=10 \mathrm{~nm}$, as shown in Fig. 14(a) by comparison to spheres with $a=5 \mathrm{~nm}$ (solid curves). ${ }^{12}$ Spectral features associated with dipolar $(l$ $=1)$ and quadrupolar $(l=2)$ excitations are clearly discernible in the spectra. Higher-order multipoles produce a shoulder piling up near $\omega_{s}$ because their separation is smaller than the absorption width. In addition, the dipole feature is broader in the larger sphere, which is again a retardation effect, since the coupling of this mode to emitted light increases with the particle dipole strength (i.e., with particle size), therefore limiting the lifetime of the dipolar plasmon due to radiative decay (Myroshnychenko, Rodríguez-Fernández, et al., 2008). This point is further discussed in Sec. IV.F.2.

For sufficiently small particles and aloof trajectories $(b>a)$, we can retain dipole terms $(l=1)$, in which case Eq. (33) reduces to

\footnotetext{
${ }^{12}$ Loss and CL emission probabilities are plotted per unit of lost-energy range (i.e., in $\mathrm{eV}^{-1}$ ), so that the areas under the spectral curves of Fig. 14(a) yield dimensionless probabilities per electron.
}
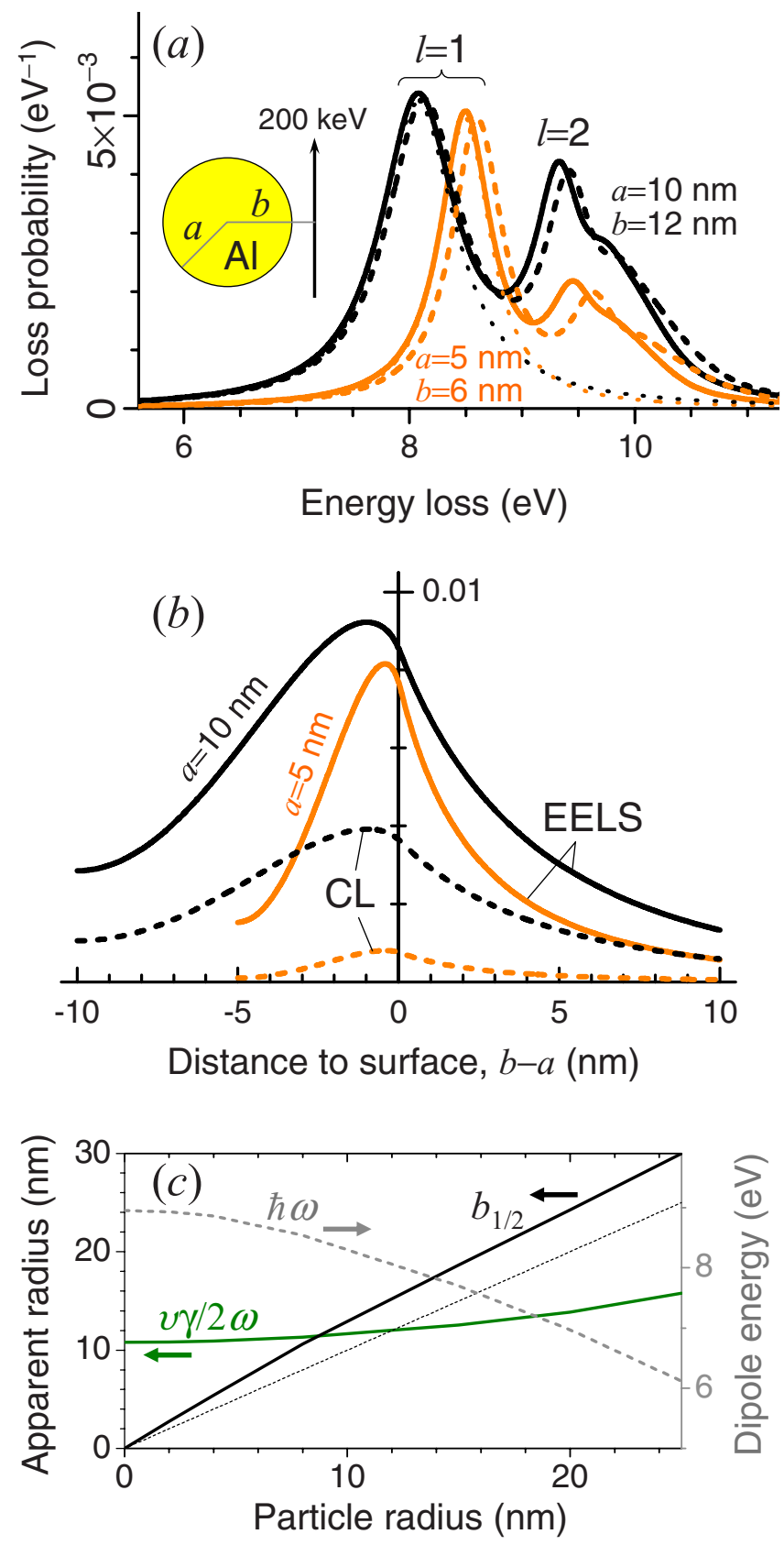

FIG. 14. (Color online) Plasmon losses and spatial resolution in Al spheres. (a) The loss probability is calculated for two different particle sizes, exhibiting several multipole plasmons that redshift as the particle radius increases. Solid curves: Result of Eq. (33) using local response. Dashed curves: Same equation with nonlocal scattering coefficients (see Appendix C). Dotted curves: Prediction of Eq. (34) (dipole approximation). The $\mathrm{Al}$ is described by the dielectric function of Eq. (A1) with $\hbar \omega_{p}=15 \mathrm{eV}$ and $\hbar \eta=0.5 \mathrm{eV}$. (b) Impact-parameter dependence of the dipolar mode maximum. (c) Apparent radius of the dipole excitation in an $\mathrm{Al}$ sphere, modeled within local response and defined as the impact parameter $b_{1 / 2}$ for which the EELS probability of a $200 \mathrm{keV}$ electron is half the maximum as a function of $b$. The dipole-energy dependence on particle size (right scale) is taken into account. The $v \gamma / 2 \omega$ distance of exponential decay in $\Gamma_{\text {EELS,dip }}[$ Eq. (34)] is shown for comparison. 


$$
\Gamma_{\text {EELS,dip }}(\omega)=\frac{1}{\hbar \pi}\left(\frac{2 e \omega}{v^{2} \gamma}\right)^{2} f\left(\frac{\omega b}{v \gamma}\right) \operatorname{Im}\{\alpha\}
$$

where

$$
f(\zeta)=K_{1}^{2}(\zeta)+K_{0}^{2}(\zeta) / \gamma^{2}
$$

Actually, this approximation works quite well even for relatively large spheres, as shown by the dotted curves of Fig. 14(a), obtained from Eq. (34) with the electric polarizability

$$
\alpha=3 t_{1}^{E} / 2 k^{3}
$$

$\left[t_{1}^{E}\right.$ is a Mie scattering coefficient; see Eq. (C2)]. The dipole mode is given correctly, although higher multipoles are obviously missed. In contrast to the particlesize-independent dipole-plasmon energy predicted by Eq. (32), we find that Eq. (36) incorporates retardation effects, leading to a sizable dipole-plasmon redshift with increasing particle diameter.

Spheres offer an excellent playground to discuss Coulomb delocalization effects (see Sec. III.A). As anticipated by Eq. (4) for the external electron field, Eq. (33) predicts an exponential decay of the EELS signal with $b$ and an attenuation distance given by $v \gamma / 2 \omega$. This is true when the argument of the Bessel functions is large compared to unity, a condition that is not satisfied near small spheres, in which those functions have a steeper dependence on $b$ (see Sec. II.A and Fig. 6). This is actually observed in the $b$ dependence of the loss probability shown in Fig. 14(b) (solid curves) for the dipole peaks of the spheres considered in Fig. 14(a). The probability decreases by a factor of 2 at a distance of $\sim 2 \mathrm{~nm}$ away from the surface of the smaller sphere, so that its apparent radius $b_{1 / 2} \sim 7 \mathrm{~nm}$ is significantly smaller than $v \gamma / 2 \omega \sim 12 \mathrm{~nm}$. The sphere size dependence of $b_{1 / 2}$ [Fig. 14(c)] fully corroborates these arguments.

Small metallic particles exhibit further peak shifts originating in quantum confinement and spatial dispersion, the effect of which is to break down the hypothesis of local response. Nonlocal effects in the performance of bounded metals can be easily included using the specular-reflection-model approximation (Ritchie and Marusak, 1966; Wagner, 1966; García de Abajo, 2008). Then, Eq. (31) is still valid if we replace $\epsilon$ by (Rojas $e t$ al., 1988)

$$
\epsilon_{l}(\omega)=\frac{\pi}{2 a(2 l+1)}\left(\int_{0}^{\infty} \frac{d q}{\epsilon(q, \omega)} j_{l}^{2}(q a)\right)^{-1}
$$

in the polarizability of Eq. (32), where $\epsilon(q, \omega)$ is the nonlocal bulk response discussed in Secs. III.B and A.1, and $j_{l}$ is a spherical Bessel function. This level of description is similar to the work of Fujimoto and Komaki (1968). Under the conditions of Fig. 14(a), however, one needs to simultaneously include nonlocal and retardation effects, for which the above procedure is not enough.

The hydrodynamic model described in Appendix A.2 incorporates both spatial-dispersion and retardation effects. When applied to spherical particles, it still yields analytical expressions for the resulting nonlocal Mie scattering coefficients, which we introduce in Appendix C. Using these coefficients in combination with Eq. (33), we predict blueshifts with respect to the local descriptions in the plasmons of the Al particles of Fig. 14(a) (dashed curves) even for relatively large spheres. This type of dispersion operates on a length scale comparable to the wavelength of conduction electrons at the Fermi level, which is of the order of $0.5 \mathrm{~nm}$ for typical metals. This is consistent with the large nonlocal effect observed for the quadrupole as compared to the dipole in the $a$ $=5 \mathrm{~nm}$ sphere since the $l=2$ mode involves more sign changes in the induced surface charge that increase the relative importance of short distances. It should be noted that spatial dispersion has been found to be partially compensated by the smooth electron density profile at the particle surface (Ruppin, 1978).

In this regard, nonlocality is important at distances $\sim v_{F} / \omega$ (Larkin et al., 2004; Larkin and Stockman, 2005), as derived from the minimum momentum transfer in the $e-h$ pair region for a given excitation frequency $\omega$ (see Fig. 8). This is the so-called Landau damping, which has been phenomenologically accounted for through a sizedependent local dielectric function, with the dephasing rate increased by $\sim a / v_{F}$ (Batson, 1980; Hövel et al., 1993; Kreibig and Vollmer, 1995). Nonlocal effects in noble metal particles are noticeable up to a size $\sim 10-20 \mathrm{~nm}$ at optical frequencies, but can be important in other materials for larger sizes $\sim 100 \mathrm{~nm}$ [e.g., silver halide nanocrystals, studied with EELS by Oleshko (2008)].

There are only scarce data on plasmon dispersion in small spheres [see, for example, Ouyang et al. (1992)] and particularly on the spatial distribution of these effects, so there is a clear need for further EELS experiments that can shed more light into this subject, which is becoming increasingly important in many nanophotonics developments that make use of metallic nanoparticles in the sub-20-nm range. We hope that these results, together with the dimer calculations of Sec. V.E, contribute to trigger future research in this direction.

\section{Coupled nanoparticles}

Coupling between plasmon modes in neighboring nanoparticles has attracted considerable interest in the electron microscopy community. Early energy-loss experiments focused on the interaction between neighboring spheres (Batson, 1982b, 1985), leading to hybridization of individual particle plasmons, the energies of which were successfully explained through analytical derivations. Further theoretical work addressed these modes in more detail (Ruppin, 1982; Schmeits and Dambly, 1991), and eventually the coupling of the electron to two neighboring spheres was derived in the nonretarded approximation (Zabala et al., 1997).

The interaction between more than two nonaligned particles is difficult to explore by analytical techniques. A particularly suitable method that yields highly convergent results, including retardation, consists in expanding the electromagnetic field in terms of multipoles around 
the particles and then allowing these multipoles to propagate among particles, thus generating a selfconsistent field (García de Abajo, 1999b). More details on this method are given in Sec. III.E.4.

Cylinder pairs have been theoretically addressed as well in the nonretarded limit by Schmeits (1989). This study was later extended to include retardation and to cope with more than two cylinders (García de Abajo and Howie, 2002; Manjavacas and García de Abajo, 2009).

Much work has been devoted in recent years to the interaction between metallic nanoparticles in the context of nanophotonics due in part to the strong enhancement of the light intensity in the interparticle region at close proximity (Xu et al., 1999). This has direct application to biosensing using surface-enhanced Raman scattering (Talley et al., 2005). Furthermore, the plasmon modes of neighboring particles can be tuned to achieve on-demand frequencies by varying their separation, as shown in optical measurements performed on lithographically printed metal dots (Atay et al., 2004). However, there are still many issues that remain unclear in the interaction between particles, especially when the gap distance is of the order of a few nanometers. Among them, the role of Landau damping and other dispersion effects. Energy-loss spectroscopy turns out again to be ideally suited for exploring these situations, in which nanometer spatial resolution is needed to resolve optical excitations. For example, the singular transition in the response of touching and nontouching particle dimers (Romero et al., 2006) has already prompted EELS measurements (N'Gom et al., 2008; Chu et al., 2009), although a comprehensive experimental study of this transition is still pending.

\section{E. More complex shapes}

The real world is generally more complex than planar, cylindrical, or spherical surfaces. There are numerous experimental studies of EELS involving more complicated geometries, such as Si tips (Reed et al., 1999), metallic particles supported by dielectric (Wang and Cowley, 1987a, 1987d) or by metal (Wang and Cowley, 1987b, 1987d) substrates, $\mathrm{MgO}$ wedges (García de Abajo and Aizpurua, 1997; Aizpurua et al., 1999), $\mathrm{MoS}_{2}$ platelets (Cohen et al., 2003), 2D photonic crystals (García de Abajo et al., 2003), optical modes in individual nanowires (Arslan et al., 2009), Ag spheroids (Little et al., 1984), Ag nanotriangles (Nelayah, Kociak, et al., 2007; Nelayah, Stéphan, et al., 2007; Nelayah et al., 2009), Ag nanoshells (Eggeman et al., 2007), Ag nanoholes (Sigle et al., 2009), and gold nanoparticles ranging from single nanospheres and nanorods (Bosman et al., 2007; Schaffer et al., 2009) to touching dimers and interacting nanorods (N'Gom et al., 2008; Chu et al., 2009). The corresponding theoretical efforts needed to explain these measurements involve more elaborate analytical developments as well as numerical simulations based on either Poisson's equation in the nonretarded approximation or Maxwell's equations if full retardation effects are necessary. These methods in general use local response functions and assume sharp boundaries separating different dielectric phases.

\section{Analytical methods}

Several analytical studies of EELS have been carried out in the nonretarded approximation for geometries that can be naturally described using coordinate systems in which the Poisson equation is separable.

\section{a. Ellipsoids}

The electrostatic modes of ellipsoids have been known for a long time, including analytical expressions for the polarizability (Gans, 1912; Jones, 1945). In particular, the EELS probability has been worked out for oblate ellipsoids by Illman et al. (1988).

\section{b. Wedges}

Analytical solutions have been reported for the modes of sharp wedges (Dobrzynski and Maradudin, 1972; Davis, 1976), parabolic wedges (Eguiluz and Maradudin, 1976), and hyperbolic wedges (Davis, 1976). The latter display a discrete spectrum that suffers a singular transition in the limit to a sharp edge, characterized by a continuous spectrum. The EELS probability has been calculated for parabolic wedges (García-Molina, GrasMartí, and Ritchie, 1985) with the beam running parallel to the edge.

\section{c. Supported particles}

A sphere or a hemisphere intersected by a planar surface can be described by mode matching, consisting in expanding the potential into spherical harmonics and plane waves within different regions of the structure, thus generating a set of self-consistent linear equations upon application of the boundary conditions. This method, pioneered by Zaremba (1985) to explore the van der Waals interaction of an atom with a surface feature, was later applied by Wang and Cowley (1987a) to study plasmon modes of supported particles, which they measured in $\mathrm{Al}$ spheres sitting on $\mathrm{AlF}_{3}$ (Wang and Cowley, 1987a) and Al (Wang and Cowley, 1987b) surfaces. They incorporated nonlocal effects within the hydrodynamic approximation (Wang and Cowley, 1987c). Further work on this geometry focused on calculating the loss probability (Aizpurua et al., 1996; Rivacoba et al., 1992).

Several other geometries in which Poisson's equation is separable are still unresolved, but the list of structures that can be accessed with analytical tools is short and we need in general to rely on numerical methods for more complicated shapes. We briefly discuss two of them in this section, and a third one is presented in Sec. III.G.

\section{Nonretarded boundary element method}

The boundary element method (BEM) consists in expressing the fields inside each homogeneous region of a given structure in terms of interface sources that are 
then self-consistently calculated by imposing the continuity of the potential and the electric displacement. This has the advantage that it requires a modest numerical effort since it involves parametrization of boundaries rather than volumes.

The BEM can be traced back to Maxwell, who utilized it to calculate capacitances (Maxwell, 1891). It was pioneered by Fuchs (1975) in the computer age to simulate modes of ionic cubes and later by $\mathrm{Lu}$ and Maradudin (1990) to find plasmons propagating inside channels cut into otherwise planar surfaces. Its debut in EELS was made by Ouyang and Isaacson (1989b), who obtained loss probabilities that they applied to study a particle supported on a substrate (Ouyang and Isaacson, 1989a). Subsequent developments focused on geometries characterized by either axial symmetry or translational invariance along a certain direction, thus permitting us to access a large number of complex morphologies with moderate computational effort (García de Abajo and Aizpurua, 1997) since only curves rather than surfaces need to be parametrized in those cases. This approach gives excellent results as compared to experiment for the losses of electrons passing near $\mathrm{Si}$ tips (Reed et al., 1999) or close to the edge of a $\mathrm{MgO}$ cube with different orientations of the trajectory (Aizpurua et al., 1999).

In the nonretarded BEM, the potential inside a given homogeneous region of space is separated into two terms,

$$
\phi=\phi^{\mathrm{ext}}+\phi^{\mathrm{ref}},
$$

the first of which describes the potential produced by the external charges present in one of the media, treating it as if it was infinitely extended, while the second term accounts for reflection at the boundary. Assuming an inhomogeneous structure composed of two materials with dielectric functions $\epsilon_{1}$ and $\epsilon_{2}$, respectively, the interface $S$ can be assimilated to an induced surface charge $\sigma$, so that the reflected potential becomes

$$
\phi^{\mathrm{ref}}(\mathbf{r})=\int_{S} d \mathbf{s} \frac{\sigma(\mathbf{s})}{|\mathbf{r}-\mathbf{s}|} .
$$

Inserting these expressions into Poisson's equation, one finds the following self-consistent relation for the induced boundary charge (García de Abajo and Aizpurua, 1997):

$$
\Lambda \sigma(\mathbf{s})=\mathbf{n}_{\mathbf{s}} \cdot \boldsymbol{\nabla} \phi^{\mathrm{ext}}(\mathbf{s})+\int d \mathbf{s}^{\prime} F\left(\mathbf{s}, \mathbf{s}^{\prime}\right) \sigma\left(\mathbf{s}^{\prime}\right),
$$

where

$$
\begin{aligned}
& F\left(\mathbf{s}, \mathbf{s}^{\prime}\right)=-\frac{\mathbf{n}_{\mathbf{s}} \cdot\left(\mathbf{s}-\mathbf{s}^{\prime}\right)}{\left|\mathbf{s}-\mathbf{s}^{\prime}\right|^{3}}, \\
& \Lambda=2 \pi\left(\epsilon_{2}+\epsilon_{1}\right) /\left(\epsilon_{2}-\epsilon_{1}\right),
\end{aligned}
$$

and $\mathbf{n}_{\mathbf{s}}$ is the interface normal directed toward medium 2 (see Fig. 15). The right-hand side of Eq. (38) is actually the opposite of the electric field along the boundary normal, with the integral describing the field produced by

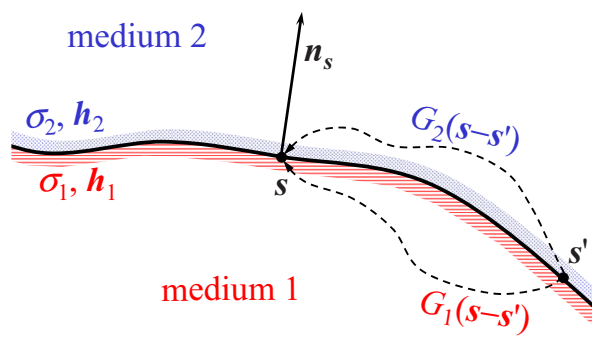

FIG. 15. (Color online) Scheme of parametrization used in the boundary element method.

induced interface charges. Equation (38) admits a representation in terms of the eigenvectors of $F$, with real eigenvalues denoted as $2 \pi \lambda_{j}$ (it can be shown that $\left|\lambda_{j}\right|$ $<1)$. This leads to the following general expression for the loss probability (García de Abajo and Aizpurua, 1997):

$$
\Gamma_{\mathrm{ref}}^{\mathrm{NR}}(\omega)=\sum_{i, j} \operatorname{Im}\left\{-A_{i j}\left(\frac{2}{\left(1+\lambda_{j}\right) \epsilon_{1}+\left(1-\lambda_{j}\right) \epsilon_{2}}-\frac{1}{\epsilon_{i}}\right)\right\},
$$

where $i$ runs over the materials 1 and 2 and $A_{i j}$ are real coupling constants that depend on the actual sample geometry and electron trajectory but not on $\epsilon_{1}$ or $\epsilon_{2}$. The second term inside the large parentheses of Eq. (39) gives a negative loss probability corresponding to the begrenzungseffekt, the weight of which increases with $A_{i j}$ in the same proportion as the interface mode described by the first term.

\section{Retarded boundary element method}

We can follow a similar procedure to incorporate retardation by solving Maxwell's equations at the expense of a sixfold increase in computational cost (García de Abajo and Howie, 1998, 2002). First of all, the electric field can be expressed in terms of vector and scalar potentials as

$$
\mathbf{E}=i k \mathbf{A}-\boldsymbol{\nabla} \phi .
$$

We find it convenient to work in the Lorentz gauge, in which $\nabla \cdot \mathbf{A}=i k \epsilon \phi$. Then the potentials become

$$
\phi(\mathbf{r})=\phi^{\mathrm{ext}}(\mathbf{r})+\int_{S_{j}} d \mathbf{s} G_{j}(|\mathbf{r}-\mathbf{s}|) \sigma_{j}(\mathbf{s})
$$

and

$$
\mathbf{A}(\mathbf{r})=\mathbf{A}^{\mathrm{ext}}(\mathbf{r})+\int_{S_{j}} d \mathbf{s} G_{j}(|\mathbf{r}-\mathbf{s}|) \mathbf{h}_{j}(\mathbf{s})
$$

inside each homogeneous region $j$ delimited by a boundary $S_{j}$, where

$$
G_{j}(r)=\frac{\exp \left(i k r \sqrt{\epsilon_{j}}\right)}{r}
$$

is the Green's function of the Helmholz equation inside that medium, $\phi^{\text {ext }}$ and $\mathbf{A}^{\text {ext }}$ are the potentials produced 
by external sources in an infinite homogeneous medium described by a dielectric function $\epsilon_{j}$, and $\sigma_{j}$ and $\mathbf{h}_{j}$ are auxiliary interface charges and currents (see Fig. 15). The boundary conditions of the electromagnetic field are then imposed and one is left with a set of selfconsistent surface integral equations that determine the boundary sources. For example, the continuity of the scalar potential leads to

$$
\begin{aligned}
& \int d \mathbf{s}^{\prime}\left[G_{1}\left(\mathbf{s}-\mathbf{s}^{\prime}\right) \sigma_{1}\left(\mathbf{s}^{\prime}\right)-G_{2}\left(\mathbf{s}-\mathbf{s}^{\prime}\right) \sigma_{2}\left(\mathbf{s}^{\prime}\right)\right] \\
& =\phi_{2}^{\mathrm{ext}}(\mathbf{s})-\phi_{1}^{\mathrm{ext}}(\mathbf{s})
\end{aligned}
$$

where $\phi_{j}^{\text {ext }}$ is the external potential in medium $j$ evaluated at positions infinitesimally close to the interface. We proceed by discretizing the integrals in these equations through a finite number of representative points distributed on the interfaces. This leads to a linear set of equations that we solve by linear algebra techniques. Further details on the method and its generalization to an arbitrary number of different media have been given by García de Abajo and Howie (2002).

The BEM is particularly advantageous for simulation of axially symmetric samples, for which different azimuthal components $m$ having $\exp (\operatorname{im} \varphi)$ dependence on azimuthal angle are uncoupled. Then the sample surface can be parametrized with a contour line, thus effectively reducing the problem to one dimension. Similarly, only the $1 \mathrm{D}$ contour defining the geometry needs to be parametrized for structures with translational invariance along a given direction. These two special cases have been discussed by García de Abajo and Howie (2002). However, the method is general and can be applied to arbitrary boundaries, as recently shown by Myroshnychenko, Carbó-Argibay, et al. (2008).

A recent example of application of the retarded BEM is shown in Fig. 16, adapted from Nelayah, Kociak, et al. (2007), in which measured loss spectra for Ag nanoprisms [Fig. 16(a)] are compared to simulations [Fig. 16(b)] when the electron beam is aimed at the corner, the edge, or the center of the triangle. Different plasmon losses are observed at each of these positions. Moreover, one can obtain snapshots of these plasmons, as shown in the images of Figs. 16(c)-16(h), by scanning the beam over the sample area and collecting the rate of selected energy losses corresponding to the three dominant features observed in the spectra of Figs. 16(a) and 16(b). These findings have been corroborated by tip-scattering NSOM measurement (Rang et al., 2008).

The fully retarded BEM has been used to investigate the optical response of nanoparticles (Aizpurua et al., 2003; Hohenester and Krenn, 2005; Sánchez-Iglesias et al., 2006; Grzelczak et al., 2007; Kumar et al., 2008; Myroshnychenko, Rodríguez-Fernández, et al. 2008) and extended nanostructures (Blanco and García de Abajo, 2004a; Cole et al., 2007), with particular emphasis placed on the localized plasmon resonances, the coupling between atoms or molecules and nanoparticles (Blanco and García de Abajo, 2004b; Trügler and Hobenester, 2008), and the interaction of fast electrons with nanopar-
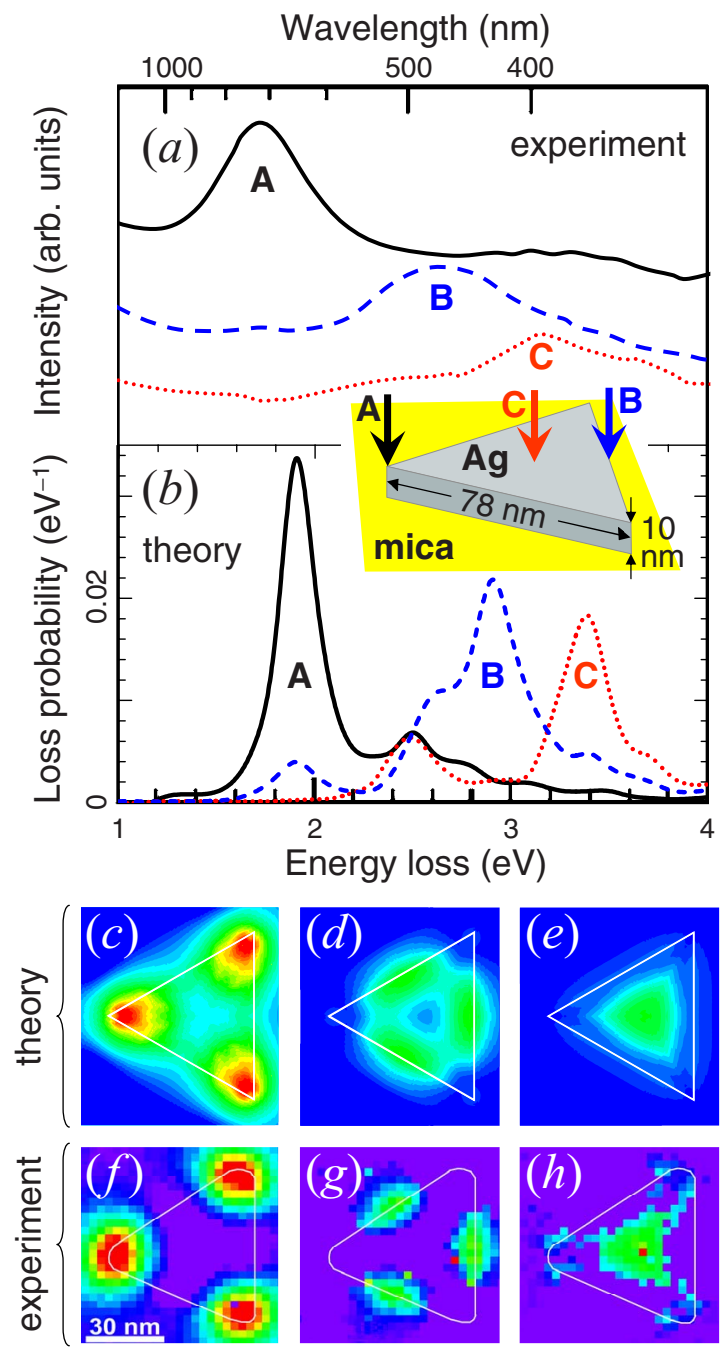

FIG. 16. (Color online) Mapping plasmon modes in an individual Ag triangular nanoprism through EELS. The triangles were supported on mica. (a) Loss spectra acquired at different positions of the electron beam over the particle (see inset). (b) Parameter-free calculated loss spectra, given in probability per incident electron per eV of energy-loss range. (c)-(e) Simulated energy-filtered images at the positions of absolute maxima in the spectra A-C, respectively. (f)-(h) Measured energy-filtered images. Adapted from Nelayah, Kociak, et al., 2007

ticles to explain EELS (García de Abajo and Howie, 1998, 2002; García de Abajo et al., 2003; Nelayah et al., 2007; Chu et al., 2009; Schaffer et al., 2009) and CL (Yamamoto, Araya, and García de Abajo, 2001; Hofmann et al., 2007; Gómez-Medina et al., 2008; Vesseur et al., 2008; Kuttge, Vesseur, Koenderink, et al., 2009) experiments.

\section{Multiple-scattering approach}

For specimens containing several neighboring particles, multiple scattering techniques provide a natural way of solving Maxwell's equations in frequency space. The field induced by each of the particles adds up to the 

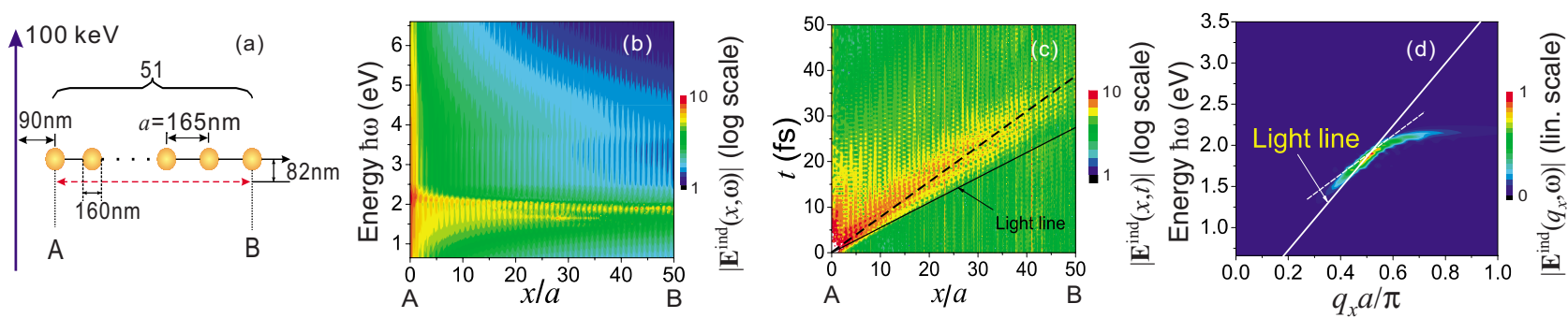

FIG. 17. (Color online) Electron-beam-induced plasmon generation in gold nanoparticle chains. (a) Scheme of the structure and geometry under consideration. (b) Induced electric field $\left|E^{\text {ind }}(x, \omega)\right|$ (in frequency space $\omega$ ) produced by the electron along the points of the $\overline{A B}$ segment [see double arrow in (a), $\| \hat{\mathbf{x}}$. (c) Same as (b) in the time domain $\left|E^{\text {ind }}(x, t)\right|$ [see Eq. (1)]. (d) Fourier transform of $E^{\text {ind }}(x, \omega)$ with respect to $x$, showing the dispersion relation of plasmons excited by the electron and propagating along the chain. The velocity of the launched pulse is found to be $\sim 0.75 c$ [dashed line in (c)], in good agreement with the group velocity at the maximum of excitation probability along the guided-mode dispersion curve [dashed line in (d)]. From Cai et al., 2009.

scattering produced by the other particles in a selfconsistent fashion.

In practice, we need to express the electric field around each particle $j$ in a complete basis set, for which vector spherical harmonics (Wang et al., 1993) are a convenient choice. The following specific steps are then followed to solve the electromagnetic problem in the presence of a passing electron (García de Abajo, 1999b): (1) the external field is scattered by each of the particles, giving rise to spherical outgoing waves (single-scattering contribution); (2) every outgoing multipole centered around a given object is expanded into propagating multipoles centered around each of the remaining objects; (3) these propagating multipoles are scattered by the objects around which they are centered, giving rise to outgoing waves that add up to the ones obtained after firstorder scattering of the electron field; and (4) this process is repeated iteratively, resulting in the self-consistent relation

$$
\xi_{j}^{\mathrm{ind}}=\xi_{j}^{\mathrm{ss}^{\prime}}+t_{j} \sum_{j^{\prime}} H_{j j^{\prime}} \xi_{j^{\prime}}^{\text {ind }}
$$

where $\xi_{j}^{\text {ss }}$ and $\xi_{j}^{\text {ind }}$ are vectors containing the coefficients of the outgoing-wave expansion of the single-scattering contribution and the self-consistent induced field, respectively, $H_{j j^{\prime}}$ is the propagation matrix that expresses outgoing waves centered around object $j^{\prime}$ in terms of propagating spherical waves centered around object $j$, and $t_{j}$ is the scattering matrix of object $j$, containing the scattering outgoing-wave coefficients after interaction of propagating spherical waves with that object. Analytical expressions for $t_{j}$ corresponding to homogeneous spheres are given in Appendix C. The multipolar expansions are truncated at some maximum orbital angularmomentum number $l_{\max }(\geq k a$ for particles of radius $a$ ), so that the above equations contain $L=2 N\left(l_{\max }+1\right)^{2}$ complex number variables inside $\xi^{\text {ind }}$, where $N$ is the number of particles, and the factor of 2 stands for the fact that we have electric and magnetic multipoles, similar to the situation encountered in Eq. (33) for the individual sphere. Direct inversion of the full system of equations takes a prohibitive computation time propor- tional to $L^{3}$. Instead, we can utilize a highly convergent iterative procedure based on Lanzcos' method (Haydock, 1980) to solve these equations (García de Abajo, 1999a, 1999b). These computations demand an effort proportional to $L^{2}$ and yield highly converged spectra for electrons passing near clusters of up to several hundred particles in a matter of minutes with $l_{\max } \sim 15$.

An example of application of this method is presented in Fig. 17, taken from Cai et al. (2009). A $100 \mathrm{keV}$ electron is considered to pass near the end of a linear array formed by 51 spherical gold particles [Fig. 17(a)]. The spectral distribution of the electric field $\left[\left|\mathbf{E}^{\text {ind }}(\mathbf{r}, \omega)\right|\right.$, plotted in Fig. 17(b) along a segment parallel to the array] exhibits prominent features at the localized plasmon resonances of the spheres. The time-domain picture [Fig. 17(c), obtained from Eq. (1)] shows plasmon propagation mediated by interparticle interaction. The group velocity (dashed curve) is consistent with the slope at the intensity maximum of the guided-mode dispersion relation derived from the space Fourier transform of $\mathbf{E}^{\text {ind }}(\mathbf{r}, \omega)$ [Fig. 17(d)].

\section{F. Composite materials}

A common situation arises when the sample contains composites made of a fine-scale mixture of different dielectric grains. Finding the optical response of the resulting metamaterial is not easy, especially in cases for which the actual microscopic geometry is unknown. This is even more complicated in EELS due to the local nature of the electron probe.

A first naive approach to the problem of explaining loss spectra in a composite consists in considering the appropriately weighed bulk probabilities derived from Eq. (19) for the constituents. The result is a reasonable approximation when the grains are large enough so that interface losses are small compared to the volume losses. This type of analysis has been used to map water distributions in biological specimens using valence excitations (Sun et al., 1993), which are more intense than core losses and provide similar information in this case, accompanied by lower sample damage. 
However, this approach breaks down for finer mixtures, as shown by Howie and Walsh (1991) through studies of losses in $\mathrm{Al}$ spheres created in $\mathrm{AlF}_{3}$ by the ionizing action of the electron beam. They found that the spectra were well reproduced by an expression like Eq. (39) with $\lambda_{j}=1 / 3$ (Howie and Walsh, 1991; Howie, 2003), which results from the dipolar mode of the spheres [Eq. (29) for $l=1$ ], now corrected by the bulk losses due to the begrenzungseffekt. Similar conclusions were extracted for other systems, such as $\mathrm{Ni}-\mathrm{Al}$ mixed oxides (Cadete Santos Aires et al., 1993).

The Maxwell-Garnett $(1904,1906)$ effective medium theory, which yields good results in many cases, is particularly suited to describe small spheres of a given material 2 embedded in a matrix of material 1 and works extremely well for small volume fractions of the spheres, $f \leqq 0.3$. This theory predicts an effective dielectric function

$$
\epsilon_{\mathrm{eff}}=\epsilon_{1} \frac{2(1-f) \epsilon_{1}+(1+2 f) \epsilon_{2}}{(2+f) \epsilon_{1}+(1-f) \epsilon_{2}},
$$

accounting for the spheres through their nonretarded dipolar response $\left[\alpha_{1}^{\mathrm{NR}}\right.$ in Eq. (B5)]. Not surprisingly, the $l=1$ mode of Eq. (29) $\left(2 \epsilon_{1}+\epsilon_{2}=0\right)$ is recovered from the zero of the denominator of Eq. (43) in the small- $f$ limit. The Maxwell-Garnett theory has been successfully employed by McComb and Howie (1995) to explain EELS measurements in $\mathrm{SiO}_{2}$ polymorphs of different density.

Corrections to the loss function of granular materials beyond the dipolar terms captured in Maxwell-Garnett theory have been explored (Fu et al., 1993; Fu and Resca, 1993). In particular, Barrera and Fuchs (1995) obtained a nonlocal effective dielectric function for a random system of sphere, described through their local bulk permittivity. This work was followed by the derivation of a spectral representation of an effective local response for the same system (Fuchs et al., 1996). More complicated situations have been considered, such as an aloof electron moving above ordered and disordered arrays of spheres (Mendoza et al., 1998, 1999) and self-similar structures formed by a continuous distribution of sphere sizes (Sosa et al., 2001), all of them analyzed in the nonretarded limit.

In this context, McKenzie and McPhedran (1977) and McKenzie et al. (1978) provided analytical expansions for the effective local response of cubic arrangements of spheres with inclusion of an arbitrarily large number of multipoles, which directly yield the loss function $\operatorname{Im}\left\{-1 / \epsilon_{\text {eff }}\right\}$. However, a similar derivation of EELS formulas for these structures is still pending, although they could shed light into the open discussion of nonlocal effects in metamaterials.

Retardation corrections to the effective dielectric function can be obtained from the photonic bands of periodic composites in the long-wavelength limit. This approach was followed by Pendry and Martín-Moreno (1994) to explain the $\sim 8 \mathrm{eV}$ loss feature measured by Howie and Walsh (1991) in aggregates of small aluminum particles. Their simulations were accompanied by a

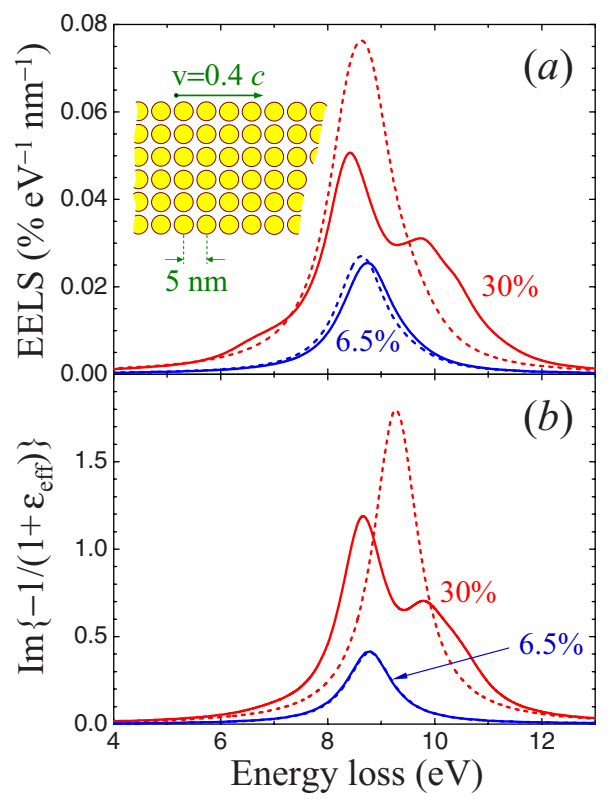

FIG. 18. (Color online) Energy loss of an electron passing near a composite material. (a) Energy-loss spectra for an electron moving parallel to the [100] direction of the (100) surface of a simple-cubic crystal formed by six layers of aluminum spheres in vacuum. The lattice period is $5 \mathrm{~nm}$. We consider two different filling fractions as shown by labels. The electron is moving at a distance of $1 \mathrm{~nm}$ from the sphere surfaces with velocity $v=0.4 c$. The dashed curves are obtained by neglecting the interaction among spheres, whereas the solid curves correspond to the full solution of Maxwell's equations using a layer KKR approach. (b) Surface loss function for the same crystal as in (a) using the effective dielectric function obtained from Maxwell-Garnett theory [broken curves, Eq. (43)] and from the layer KKR method (solid curves).

complex series of spectral features that subsequent analysis by García de Abajo and Blanco (2003) demonstrated to be due to numerical inaccuracies. In fact, the loss spectrum for small filling fraction $(f=0.06)$ of an array of equally spaced aluminum spheres already shows a single $\sim 8.5 \mathrm{eV}$ feature (García de Abajo and Blanco, 2003) [see Fig. 18(a), solid curves]. This peak splits into two for larger $f=0.3$, due to multipolar interaction between neighboring spheres, quite different from the individual-plasmon excitation predicted by MaxwellGarnett theory [Fig. 18(b), dashed curves, obtained from Eq. (43)]. Interestingly, the calculated EELS peaks in Fig. 18 are displaced to the red with respect to the maxima of the surface loss function $\operatorname{Im}\left\{-1 /\left(1+\epsilon_{\text {eff }}\right)\right\}$ as a result of retardation, similar to the effect discussed in Fig. 11. Both the EELS probability and the effective dielectric function $\epsilon_{\text {eff }}$ are calculated using a layer Korringa-Kohn-Rostoker (KKR) method, which allows solution of the multiple-scattering equation [Eq. (42)] for parallel layers of spheres (Stefanou et al., 2000; García de Abajo and Blanco, 2003). The aluminum is described by Eq. (A1) with $\hbar \omega_{p}=15 \mathrm{eV}$ and $\hbar \eta=1 \mathrm{eV}$. A value of $l_{\max }=6$ is sufficient to achieve convergence. We plot for comparison the loss probability computed in the absence of multiple-scattering interaction among 
spheres [Fig. 18(a), broken curves], predicting a single peak that is roughly independent of $f$.

\section{G. Carbon molecules and low-dimensional structures: The discrete-dipole approximation}

Carbon molecules and other low-dimensional structures have fascinated chemists and physicists since the discovery of $\mathrm{C}_{60}$ (Kroto et al., 1985) and carbon nanotubes (Iijima, 1991; Monthioux and Kuznetsov, 2006), followed by BN nanotubes (Chopra et al., 1995) and other related molecules.

Plasmon modes were first observed in solid $\mathrm{C}_{60}$ as 6.5 and $28 \mathrm{eV}$ EELS features (Gensterblum et al., 1991; Saito et al., 1991), corresponding to collective oscillations of $\pi$ and $\sigma$ electrons, respectively. The optical properties of the individual molecules is not significantly altered in solid phase since their binding interaction is basically due to weak van der Waals attraction. Actually, subsequent studies in gas phase $\mathrm{C}_{60}$ molecules corroborated these results (Keller and Coplan, 1992).

The clear-cut distinction between loosely bound $\pi$-like orbitals and tightly bound $\sigma$-like orbitals persisted in the loss spectra of carbon nanotubes (Kuzuo et al., 1992), in both single-wall and multishell configurations (Stéphan et al., 2002), in multishell fullerenes (Henrard et al., 1999), in BN nanotubes (Kociak et al., 2000), and in other similar types of molecules (Kociak et al., 2001).

These results were soon related to the dielectric properties of graphite, the anisotropic permittivity of which was used as input to describe fullerenes and nanotubes via continuous dielectric theory, with different $\epsilon$ for field components parallel and perpendicular to the carbon shells (Lucas et al., 1994). The dielectric approach yielded reasonable results compared to measurements of plasmons sustained by a diverse number of geometries and chemical identities, including multishell $\mathrm{BN}$ nanotubes, $\mathrm{C}$ nanotubes, $\mathrm{C}$ fullerenes (Henrard et al., 1999; Kociak et al., 2000; Stéphan et al., 2002), $\mathrm{WS}_{2}$ hollow nanoparticles (Kociak et al., 2001), and $\mathrm{WS}_{2}$ nanotubes (Taverna et al., 2002). Good agreement was obtained for nanotubes of different thicknesses down to the single-wall level (Stéphan et al., 2002).

First-principles calculations have also been carried out to describe the interaction of fast electrons with carbon nanotubes (Nojeh et al., 2006). The agreement with experimental loss spectra of single-wall tubes is satisfactory (Marinopoulos et al., 2003). However, this approach is currently limited to relatively small structures due to the heavy computational demand of spectroscopy $a b$ initio methods.

The discrete nature of these molecules suggests an atomistic approach to describe their response, with each atom represented by an induced dipole of different polarizability in the directions out of plane and along the tangent plane of the atomic shells. This is the so-called discrete-dipole approximation (DDA), in which the atomic polarizability is obtained from the dielectric function of the bulk material (e.g., graphite for carbon nanotubes and fullerenes) by inverting the Clausius-
Mossotti relation. ${ }^{13}$ A detailed comparison between the DDA and the continuous dielectric theory was carried out by Henrard and Lambin (1996), who showed that both approaches are in reasonable mutual agreement.

The DDA was pioneered by Purcell and Pennypacker (1973) in the study of the optical response of dielectric grains by dividing them into fine volume elements that were described through induced dipoles. Actually, the DDA is a versatile method for dealing with arbitrary sample morphologies and is employed to predict optical properties of nanoparticles (Draine and Flatau, 1994; Kelly et al., 2003; Noguez, 2007). This versatility should find application in the simulation of EELS, and therefore we offer a simple derivation of the method that generalizes previous approaches to fully include retardation effects.

In order to apply the DDA to carbon structures, each of the atoms (or, alternatively, each of the bonds in a similar formulation) is assimilated with an induced point particle of polarizability tensor $\alpha(\omega)$. The dipoles induced on these points in response to an external electric field $\mathbf{E}^{\text {ext }}(\mathbf{r})$ are then obtained from their self-consistent interaction, so that the dipole of the particle located at position $\mathbf{r}_{j}$ is given by

$$
\mathbf{p}_{j}=\alpha \cdot\left(\mathbf{E}^{\operatorname{ext}}\left(\mathbf{r}_{j}\right)+\sum_{j^{\prime} \neq j} \mathcal{G}_{j j^{\prime}}^{0} \cdot \mathbf{p}_{j^{\prime}}\right)
$$

where the $3 \times 3$ symmetric tensor $\mathcal{G}_{j j^{\prime}}^{0}$ describes the coupling with the dipole at $\mathbf{r}_{j^{\prime}}{ }^{14}$ Equation (44) can be solved by direct matrix inversion to yield

$$
p=\frac{1}{\alpha^{-1}-\mathcal{G}^{0}} \cdot E^{\mathrm{ext}}
$$

where $p$ represents the vector formed by the components of all dipoles $\mathbf{p}_{j}$, the matrix $\mathcal{G}^{0}$ is made of the components of $\mathcal{G}_{j j^{\prime}}^{0}$, and so on. The electric field corresponding to a passing electron is given by Eq. (4), that is, $\mathbf{E}^{\operatorname{ext}}\left(\mathbf{r}_{j}\right)=\left(2 e \omega / v^{2} \gamma\right) \mathbf{g}_{j}$, where $\mathbf{g}_{j}=\mathbf{g}\left(\mathbf{r}_{j}\right)$ [see Eq. (5)].

\footnotetext{
${ }^{13}$ For carbon structures, the effective polarizability per atom is obtained from the dielectric function of graphite using $\alpha_{i}^{-1}$ $=\left(4 \pi n_{\mathrm{C}} / 3\right)\left[\left(\epsilon_{i}+2\right) /\left(\epsilon_{i}-1\right)-S_{i}\right]$, where $n_{\mathrm{C}}=113.63 \mathrm{~nm}^{-3}$ is the atomic density of this material, $i=\|, \perp$ refers to the response to an electric field either parallel or perpendicular to the carbon planes, and the constants $S_{\perp}=-2 S_{\|}=2.8216$ originate in the lack of inversion symmetry of the graphite atomic lattice (Ashcroft and Mermin, 1976).

${ }^{14}$ The electric field created by dipole $\mathbf{p}_{j^{\prime}}$ is given by

$$
\begin{aligned}
\mathcal{G}_{j j^{\prime}}^{0} \cdot \mathbf{p}_{j^{\prime}}= & \frac{e^{i k u}}{u^{3}}\left(\left[(k u)^{2}+i k u-1\right] \mathbf{p}_{j^{\prime}}\right. \\
& \left.-\left[(k u)^{2}+3 i k u-3\right] \frac{\left(\mathbf{u} \cdot \mathbf{p}_{j^{\prime}}\right) \mathbf{u}}{u^{2}}\right),
\end{aligned}
$$

where $\mathbf{u}=\mathbf{r}_{j}-\mathbf{r}_{j^{\prime}}$. This quantity is related to the Green's tensor $G$ of Eq. (12) in vacuum through $\mathcal{G}^{0}=-4 \pi \omega^{2} G$.
} 


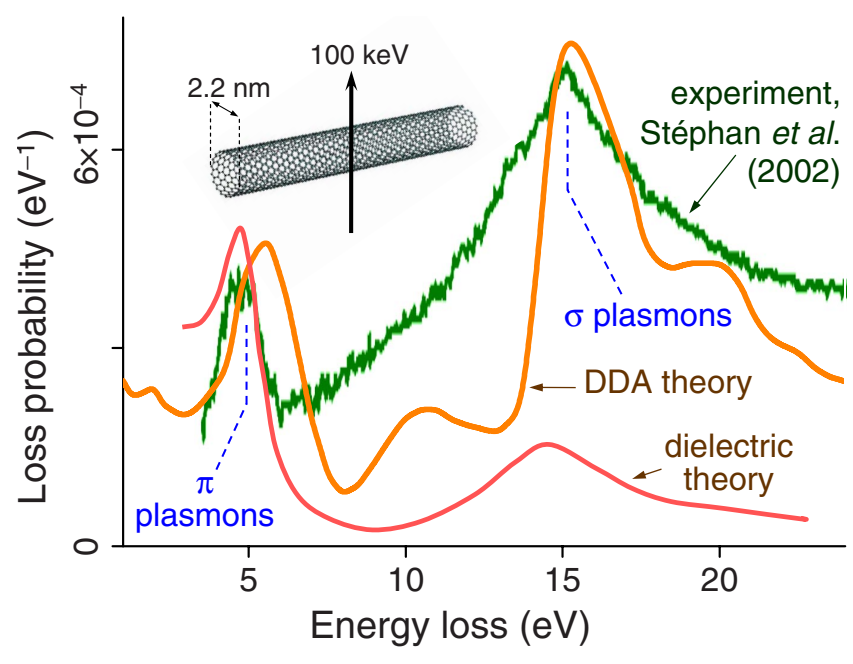

FIG. 19. (Color online) Probing plasmons in a single-wall carbon nanotube via EELS. The experiment shows prominent $\pi$ and $\sigma$ plasmons that are relatively well described both by the discrete-dipole approximation (DDA) and by a dielectric approach. The experimental curve and the dielectric theory are from Stéphan et al., 2002. The DDA calculation performed here for a $16 \times 16$ nanotube is based on the dielectric function of graphite, taken from Palik, 1991.

Using the same matrix notation as in Eq. (45), the fully retarded loss probability in the presence of our polarizable point particles representing carbon atoms reads

$$
\Gamma_{\mathrm{EELS}}(\omega)=\frac{1}{\hbar \pi}\left(\frac{2 e \omega}{v^{2} \gamma}\right)^{2} \operatorname{Im}\left\{g^{+} \cdot \frac{1}{\alpha^{-1}-\mathcal{G}^{0}} \cdot g\right\} .
$$

Of course, this expression reduces to Eq. (34) in the single-atom case $\left(\mathcal{G}^{0}=0\right)$.

As an example of application of Eq. (46), Fig. 19 shows the loss probability for an electron passing near a single-wall carbon tube under grazing incidence conditions. The experimental data and the results of the anisotropic dielectric theory are taken from Stéphan et al. (2002). The position of the plasmon features predicted by the above DDA formalism compares well to both of them. It is remarkable that dielectric theory is still producing meaningful results for such thin nanotubes, although the DDA seems to attribute a relative weight to $\pi$ and $\sigma$ plasmons in better agreement with the observed spectrum.

\section{H. Relation to the photonic local density of states}

We can ask ourselves how exactly the loss probability is related to the photonic properties of the sample and whether it is possible to retrieve from measured data for bounded media a relevant quantity similar to the dielectric function. This question has a positive answer in systems with translational invariance along the direction of electron motion: the EELS probability is proportional to the photonic local density of states (LDOS) (García de Abajo and Kociak, 2008b). ${ }^{15}$

The photonic LDOS is defined by analogy to its electronic counterpart in solid-state physics as the combined local intensity of all normalized eigenmodes of the system (Ashcroft and Mermin, 1976), but unlike electronic wave functions the electric field is a vectorial quantity, which suggests defining separate Cartesian projections of the LDOS along unit vectors $\hat{\mathbf{n}}$ as (Fussell et al., 2005)

$$
\rho_{\hat{\mathbf{n}}}(\mathbf{r}, \omega)=-\frac{2 \omega}{\pi} \operatorname{Im}\{\hat{\mathbf{n}} \cdot G(\mathbf{r}, \mathbf{r}, \omega) \cdot \hat{\mathbf{n}}\},
$$

in terms of the Green's tensor defined in Eq. (12). ${ }^{16}$ The uniform LDOS in free space is known from black-body theory (Loudon, 2000),

$$
\rho_{\hat{\mathbf{n}}}^{0}(\mathbf{r}, \omega)=\omega^{2} / 3 \pi^{2} c^{3},
$$

which contains an additional factor of $1 / 3$ arising from the projection over a specific Cartesian vector $\hat{\mathbf{n}}$.

If we choose the direction of translation invariance along the $\hat{\mathbf{z}}$ axis, the Green's tensor $G\left(\mathbf{r}, \mathbf{r}^{\prime}, \omega\right)$ depends on $z$ and $z^{\prime}$ only via $z-z^{\prime}$, and consequently we can write

$$
G\left(\mathbf{r}, \mathbf{r}^{\prime}, \omega\right)=\int \frac{d q_{z}}{2 \pi} \tilde{G}\left(\mathbf{R}, \mathbf{R}^{\prime}, q_{z}, \omega\right) e^{i q_{z}\left(z-z^{\prime}\right)} .
$$

Using this in Eq. (13), the loss probability can be recast as

$$
\begin{aligned}
\Gamma_{\mathrm{EELS}}\left(\mathbf{R}_{0}, \omega\right) & =\frac{4 e^{2} L}{\hbar} \operatorname{Im}\left\{-\tilde{G}_{z z}\left(\mathbf{R}_{0}, \mathbf{R}_{0}, q_{z}, \omega\right)\right\} \\
& =\frac{2 \pi e^{2} L}{\hbar \omega} \tilde{\rho}_{\hat{\mathbf{z}}}\left(\mathbf{R}_{0}, q_{z}, \omega\right),
\end{aligned}
$$

\footnotetext{
${ }^{15}$ There is a univocal correspondence between the spatially dependent local dielectric function and the Green's tensor, as can be deduced upon inspection of Eq. (12). This correspondence works frequency by frequency. Furthermore, the photonic LDOS is directly obtained from the Green's tensor following Eq. (47). The inverse relation is less direct, although one can extend the equivalent of the Hohenberg and Kohn (1964) theorem to light, thus asserting that there is a univocal correspondence between dielectric functions and LDOS, with both quantities defined within a finite frequency range above $\omega=0$ (i.e., the correspondence is not local in frequency).

${ }^{16}$ The definition of Eq. (47) assumes that the photon eigenmodes are well defined (that is, in the absence of absorption) (Colas des Francs et al., 2001). An alternative interpretation, which holds even in the presence of lossy materials (D'Aguanno et al., 2004), comes from the realization that $\left(4 \pi^{2} \omega D^{2} / \hbar\right) \rho_{\hat{\mathbf{n}}}$ gives the spontaneous decay rate for an excitation dipole strength $D$ (Barnett and Loudon, 1996; Fussell et $a l ., 2004)$. It should also be noted that a complete definition of the LDOS must include a magnetic part (Joulain et al., 2003), which is, however, uncoupled to our fast electrons.
} 
where $q_{z}=\omega / v$ is determined by energy conservation [see Eq. (3)], $L$ is the length of the trajectory, and

$$
\tilde{\rho}_{\hat{\mathbf{n}}}\left(\mathbf{R}, q_{z}, \omega\right)=-\frac{2 \omega}{\pi} \operatorname{Im}\left\{\hat{\mathbf{n}} \cdot \tilde{G}\left(\mathbf{R}, \mathbf{R}, q_{z}, \omega\right) \cdot \hat{\mathbf{n}}\right\}
$$

is the photonic density of states, local in real space along the $\mathbf{R}$ directions perpendicular to the beam and local in wave-vector space $q_{z}$ along the direction of motion, $\hat{\mathbf{z}}$. This relation between the photonic LDOS and the EELS probability is further illustrated in subsection of Sec. IV.D for electrons moving inside 2D photonic crystals.

For a nonlossy homogeneous dielectric of real, positive permittivity $\epsilon$, one finds $\tilde{\rho}_{\hat{\mathbf{z}}}\left(\mathbf{R}, q_{z}, \omega\right)=\left(\omega / 2 \pi c^{2}\right)(1$ $\left.-q_{z}^{2} / k^{2} \epsilon\right) \theta\left(k^{2} \epsilon-q_{z}^{2}\right)$, which reflects the fact that only electrons moving faster than light in the material can lose energy [see Eq. (7)]. This is the Cherenkov effect that we discuss in Sec. IV.D. As expected, the vacuum density of states does not contribute to the EELS signal.

Equation (49) constitutes a rigorous relation between the EELS probability and the LDOS valid for $2 \mathrm{D}$ systems with translational invariance along the direction of electron motion. The LDOS enters this relation through its projection over specific components $q_{z}$ of light wave vector along that direction. A similar relation can be obtained for fully arbitrary geometries from Eq. (13) (García de Abajo and Kociak, 2008b),

$$
\begin{aligned}
\Gamma_{\mathrm{EELS}}\left(\mathbf{R}_{0}, \omega\right) & =\frac{4 e^{2}}{\hbar} \operatorname{Im}\left\{-G_{z z}^{\mathrm{ind}}\left(\mathbf{R}_{0}, \mathbf{R}_{0}, q_{z},-q_{z}, \omega\right)\right\} \\
& =\frac{2 \pi e^{2}}{\hbar \omega} \rho_{\hat{\mathbf{z}}}\left(\mathbf{R}_{0}, q_{z}, \omega\right),
\end{aligned}
$$

where $G_{z z}^{\text {ind }}\left(\mathbf{R}, \mathbf{R}^{\prime}, q_{z},-q_{z}^{\prime}, \omega\right)$ is the Fourier transform of the induced Green's tensor with respect to $z$ and $z^{\prime}, q_{z}$ $=\omega / v$, and we have defined, by analogy to Eq. (47), the quantity

$$
\rho_{\hat{\mathbf{n}}}(\mathbf{R}, q, \omega)=-\frac{2 \omega}{\pi} \operatorname{Im}\{\hat{\mathbf{n}} \cdot G(\mathbf{R}, \mathbf{R}, q,-q, \omega) \cdot \hat{\mathbf{n}}\}
$$

as a generalized density of states that is local in real space along the $\mathbf{R}$ directions and local in momentum space along the remaining $z$ direction. In $2 \mathrm{D}$ systems, we have $\rho_{\hat{\mathbf{n}}}=L \tilde{\rho}_{\hat{\mathbf{n}}}$.

Unlike the $2 \mathrm{D}$ case, a rigorous simple relation between EELS and the LDOS as defined by Eq. (47) has not been yet reported for arbitrary 3D systems. Although some numerical results point out qualitative similarities between both quantities in certain geometries (García de Abajo and Kociak, 2008b), it has been recently argued that they differ substantially in general (and particularly near particle gaps, in which electricfield enhancement occurs for directions roughly perpendicular to the electron trajectory) because the electron charge and its stopping via the induced field have a delocalized nature, compared to the fields of the point dipole in terms of which the 3D LDOS of Eq. (47) is defined (Hohenester et al., 2009).
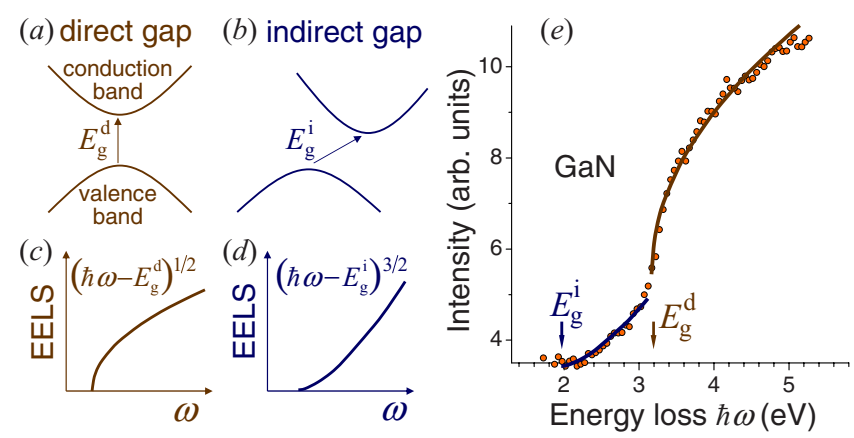

FIG. 20. (Color online) Gap determination using EELS. (a) and (b) Direct and indirect gaps. (c) and (d) Loss probability near direct and indirect gaps. Adapted from Rafferty and Brown, 1998. (e) Example of simultaneous fit of indirect and direct gaps in $\mathrm{GaN}$ for $E_{\mathrm{g}}^{\mathrm{i}}=2 \mathrm{eV}$ and $E_{\mathrm{g}}^{\mathrm{d}}=3.2 \mathrm{eV}$, adapted from Lazar et al., 2003.

\section{Electronic structure determination}

Publications containing EELS studies of local electronic structures are so numerous that only a succinct review is possible here. A spectacular degree of spatial resolution is achieved by resorting to high-energy losses, for instance, when mapping $s p^{2}$ and $s p^{3}$ carbon in a silicon-diamond interface with subnanometer detail (Muller et al., 1993) and also when imaging $\mathrm{K}$ atoms in doped fullerenes (Guan et al., 2005). However, valence losses can provide excellent resolution too with minimum sample damage, as shown by Daniels et al. (2003), who mapped carbon chemical states through characteristic bulk plasmon losses with nanometer detail. In general, comparison with $a b$ initio theory greatly facilitates the identification of spectral features in the measured loss spectra (Marinopoulos et al., 2003; Keast and Bosman, 2008).

The determination of band gaps in insulators is successfully addressed by valence EELS (Dorneich et al., 1998), although this requires special care to deal with the subtraction of the ZLP and to get rid of CR losses, which can be misleading in the assessment of the electronic inelastic signal threshold (Stöger-Pollach et al., 2006, 2008; Gu et al., 2007; Stöger-Pollach and Schattschneider, 2007; Stöger-Pollach, 2008).

The loss probability has been shown to be closely related to the so-called joint density of states (JDOS) (Pflüger et al., 1984, 1985), which is the convolution of the density of states of occupied and unoccupied electronic bands. The JDOS gives an intuitive measure of the interband-transition probability when more subtle effects in the excitation matrix elements and lifetime effects are neglected. The JDOS predicts a dependence of the loss probability on the energy transfer $\hbar \omega$ as $\sim(\hbar \omega$ $\left.-E_{g}^{d}\right)^{1 / 2}$ and $\sim\left(\hbar \omega-E_{g}^{i}\right)^{3 / 2}$ at the edge of direct- and indirect-gap energies $E_{g}^{d}$ and $E_{g}^{i}$, respectively (Rafferty and Brown, 1998). A good example of this is found in GaN, as shown in Fig. 20, adapted from Lazar et al. (2003). A direct gap involves a more abrupt decay of the loss probability near the gap [Figs. 20(a)-20(d)]. Interest- 
ingly, both the smooth tail of an indirect gap and the sharper edge of a higher-energy direct gap are clearly resolved in the loss spectra of GaN [Fig. 20(e)].

In this context, Van Hove singularities (Van Hove, 1953) appearing in the electronic density of states at energies corresponding to maxima, minima, and saddle points of the bands have also been resolved using valence EELS (Dorneich et al., 1998).

Finally, the spatial resolution of electron microscopes can be employed at its best to study electronic bands of nanostructures. For instance, this has been done to measure optical gaps in single-, double-, and triple-wall BN nanotubes (Arenal et al., 2005), showing that the gap is rather independent of thickness and stays near the inplane value of hexagonal bulk $\mathrm{BN}(\sim 5.8 \mathrm{eV})$. In a different development, quantum confinement effects in the gaps of individual CdSe quantum dots have been observed using space-resolved EELS (Erni and Browning, 2007).

\section{CATHODOLUMINESCENCE: GENERATION OF LIGHT BY INCOMING ELECTRONS}

Cathodoluminescence (CL) was first observed in the mid-19th century as light emission produced when cathode rays (electrons) struck the glass of evacuated discharge tubes. Since then, a whole industry has been generated in the search for outstanding phosphorescent materials (Ozawa, 1990), and, in particular, CL has been extensively used in 20th century television screens, and even TEMs have benefited from it by projecting sample images in phosphor screens.

Cathodoluminescence offers a method for characterizing microstructures causing minimum sample damage. It can be regarded as a contactless technique under the aloof configuration (see Sec. III.C). CL is widely utilized in mineralogy (Schieber et al., 2000; Pratesi et al., 2003; Pennycook, 2008) to resolve the composition, contamination, and defects of natural structures with submicron detail. It is also a common tool to study the electronic bands of insulators (Balberg and Pankove, 1971) and semiconductors (Yacobi and Holt, 1986; Shubina et al., 2004) and particularly the effect of dopants (Holt and Yacobi, 1990). Recently, CL has been used as a means to read stored information by tracing phase transitions in Ga nanoparticles (Denisyuk et al., 2008), which can be in turn controlled by electron excitation (Pochon et al., 2004). Cathodoluminescence is typically performed in adapted SEMs using 1-50 keV beams, and it is now possible to record spectra for every pixel of a given image (Galloway et al., 2003). In a separate development, lowenergy electrons $(150-300 \mathrm{eV})$ emitted from scanning tunneling microscope tips have been employed to produce surface $\mathrm{CL}$, which benefits from the small inelastic mean free path of electrons at those energies (Ma et al., 2006). A close relative of $C L$ is found in light emission accompanying inelastic electron tunneling, which can be assisted by localized plasmon excitations (Persson and Baratoff, 1992), although the photon yield is generally low in that case.

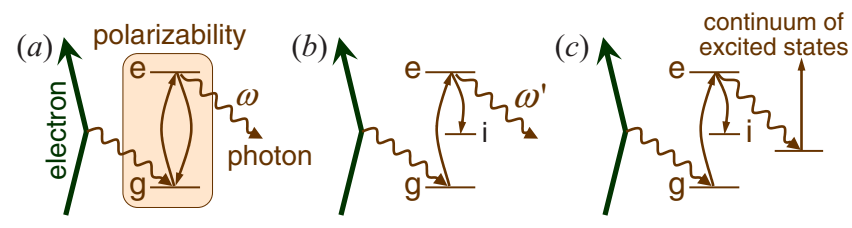

FIG. 21. (Color online) Some of the processes taking place during energy transfer from a swift electron to a sample. (a) Excitation from the specimen ground state $g$ to an excited state $e$, and subsequent radiative decay of the latter (see Sec. IV.A.1). (b) Excitation followed by incoherent radiative decay to an intermediate state $i$ (see Sec. IV.A.2). (c) Excitation followed by nonradiative decay.

Here we are interested in the application of CL to investigate the optical properties of nanostructured materials. Besides $e-h$ pair recombination, which dominates $\mathrm{CL}$ in semiconductors, we are interested in other mechanisms of light emission such as excitation of plasmons in metal nanoparticles. Overall, CL constitutes a good technique to access optical properties with nanometer resolution and without spurious effects due to modifications produced by the presence of a probe in the region near the sample, as is the case in NSOM.

\section{A. Mechanisms of light emission}

We find it convenient to classify the mechanisms of $\mathrm{CL}$ emission according to their degree of coherence with respect to the external electric field of the bombarding electrons.

\section{Coherent electron-induced radiation emission}

The interaction of the electron with a material gives rise to electromagnetic far-field components that are coherent with respect to the external evanescent field accompanying the moving charge, described by Eq. (4). These far-field components produce what we call electron-induced radiation emission (EIRE). There are several mechanisms of coherent light emission, the most notorious of which are transition radiation (Sec. IV.C), Cherenkov radiation (Sec. IV.D), and diffraction radiation (Sec. IV.E). The electric field associated with each of these mechanisms is subject to the same set of Maxwell equations, so that we must observe interference effects between them.

The interference of coherently emitted light is actually due to the fact that the quantum-mechanical state of the sample does not change after the emission process takes place. All the energy released by the electron in a scattering event giving rise to EIRE is liberated in the form of radiation, as schematically shown in Fig. 21(a).

EIRE sources have a relatively delocalized origin, since they involve regions of the sample that are large from the electronic point of view, although they can be small compared to the wavelength of the emitted light. As a consequence, the coherent photon yield increases with electron energy: the faster the charge, the more 
extended the external field (see Sec. II.A) and the more efficient the coupling to delocalized excitations.

When heavy encounters with sample atoms take place, the sudden change in the electron trajectory gives rise to radiation. This is the so-called bremsstrahlung (Jackson, 1999), which can maintain a certain degree of coherence with the sources of EIRE so far discussed. In particular, theoretical analysis has shown that bremsstrahlung produces interference with transition radiation (TR) upon electron bombardment (Ritchie et al., 1964; Shieh and Ritchie, 1970). Experimental evidence of this effect has been reported under grazing incidence conditions (Cram and Arakawa, 1967), so that deflections in the electron trajectory occur close to the surface. However, the contribution of bremsstrahlung to the total photon yield under usual TEM and SEM operation conditions is minor unless very grazing trajectories are considered (Tomaš et al., 1972, 1974).

\section{Incoherent cathodoluminescence}

The incoherent part of the emission bears close relation to photoluminescence: the sample is first excited and then it decays inelastically by emitting light of a different color, displaced to the red with respect to the external illumination. The only difference lies in the source of excitation: while external light preferentially couples to strong-dipole modes, electrons can efficiently excite metastable levels because they are capable of transferring relatively large momenta to the sample (see, for example, Fig. 8) which enhances the probability of nondipole transitions. Furthermore, the electron field can be regarded as a supercontinuum source of evanescent light, suitable for creating energetic excitations (for instance, in large-band-gap insulators) that would be difficult to produce using a laser instead.

Figure 21(b) shows a typical incoherent emission process, whereby the excited state produced by the electron decays into an intermediate state, giving rise to light of frequency $\omega^{\prime}$ below the transferred $\omega$. The sample changes its quantum-mechanical state after the interactions with the electron and the optical emission take place, and consequently the resulting light cannot produce interference with coherent EIRE. This contribution to $\mathrm{CL}$ is similar to the outcome of excited-atom decay (i.e., proportional to the radiative part of the photonic LDOS).

Incoherent emission is generally associated to the creation of $e-h$ pairs and excitons. Actually, CL is routinely used to investigate these types of excitations (Sauer et al., 2000; Koizumi et al., 2001; Rodt et al., 2005) and particularly when one is interested in resolving them in space, for instance, to study quantum dots (Grundmann et al., 1995; Rodt et al., 2005), quantum confinement in semiconductors (Chen et al., 2006; Yamamoto, Bhunia, and Watanabe, 2006), and optical effects driven by surface atomic steps (Minoda and Yamamoto, 2006).

We should expect the opposite dependence of the incoherent emission yield on electron velocity as compared to coherent EIRE. The excitations in this case are highly localized, so that the strength of the coupling to the more delocalized field of faster electrons turns out to be weaker. This effect is actually reflected in the energy dependence of the electron inelastic mean free path (EIMFP) (Tanuma et al., 1994), shown in Fig. 1: this quantity passes through a minimum at $E_{0} \sim 50 \mathrm{eV}$ electron kinetic energy, in which inelastic excitation is more probable, and exhibits a monotonic increase with $E_{0}$ due to weaker Coulomb interaction when the electron moves faster $\left(\mathrm{EIMFP} \sim E_{0} / \ln E_{0}\right)$. Incoherent $\mathrm{CL}$ can take very large values in suitably designed insulators [for example, greater than five photons per incident $4 \mathrm{keV}$ electron in $\mathrm{LaF}_{3}$ doped with rare earths (Wang, Chan, et al., 2008).

There are other situations in which the excited states involved in incoherent CL are not so localized. For instance, in emission assisted by nanoparticle plasmons. More precisely, $d$-band holes created by photoexcitation in $\mathrm{Au}$ nanocrystals have been shown to recombine nonradiatively with $s p$ electrons that give rise to excitation of intermediate particle plasmons, the decay of which results in photoluminescence intensities that are four orders of magnitude higher than in metal films (Dulkeith et al., 2004), but still insignificant compared with electronic decay channels [see Fig. 21(c)]. Nevertheless, these phenomena are relevant to understanding $\mathrm{CL}$ assisted by particle plasmons in metal clusters embedded in dielectrics (Drachsel et al., 2002; Karali et al., 2005).

Incoherent CL can be regarded as a way to deliver a localized source of light (the inelastic excitations) with which one can investigate local photonic properties. This is possible, thanks to the effect predicted by Purcell (1946): an increase in the radiative decay rate of excited nuclei coupled to a resonant circuit. The Purcell effect is quantified by a linear dependence of the light emission rate of excited atoms on the photonic LDOS (see Sec. III.H) that has been observed in numerous experiments (see Andrew and Barnes (2001), and references therein). But of course this decay channel must compete with nonradiative processes and their relative magnitudes depend on the dielectric environment. As a rule of thumb, atomic radiative decay dominates in insulators and is negligible in metals.

A microscopic quantitative analysis of incoherent $\mathrm{CL}$ is made more complicated by the excitation of hot electrons, which are in turn a source of CL. Some of these electrons escape from the sample surface, giving rise to SEE, the yield of which can reach values above 20 electrons per primary electron in some insulators at $\sim 1 \mathrm{keV}$ incidence energy (Cazaux, 2006). This high emission is due to hot charge carriers right above the insulator gap, characterized by long mean free paths and therefore larger probability of escaping from the solid (Ortega et al., 1998).

Secondary electrons of significant energy above the Fermi level are less abundant in metals because they are rapidly quenched by strong dynamical screening. Moreover, the CL emission comes from the skin-depth subsurface layer $(\sim 20 \mathrm{~nm}$ in the visible and NIR for $\mathrm{Al}$ and noble metals), which drastically erases any effects arising 
from the electron cascade that is produced by the energetic impinging probe in deeper regions.

As noted above, the incoherent CL emission must compete with other relaxation channels, such as Auger deexcitation [see Fig. 21(c)], which are relatively inefficient in insulators. However, radiative inelastic decay gives rise to a minor contribution to $\mathrm{CL}$ in metals because electronic relaxation channels are several orders of magnitude faster. Therefore, the CL signal arising from metallic nanostructures, such as the ones contemplated in plasmonics, is dominated by EIRE, the details of which we discuss in the next sections. ${ }^{17}$

\section{B. Calculation of coherent light emission}

The intensity of light emission produced by swift electrons interacting with a sample can be calculated from the induced far field. Using the retarded BEM to solve Maxwell's equations (Sec. III.E.3), we obtain the far field from the integrals of Eqs. (40) and (41) in the $k r \rightarrow \infty$ limit. The radiation is commonly produced and measured in the vacuum medium $j=1$, in which $\epsilon_{1}=1$ and $G_{1}(|\mathbf{r}-\mathbf{s}|) \approx \exp [i(k r-k \hat{\mathbf{r}} \cdot \mathbf{s})] / r$ (for $\left.k r \gg 1\right)$, with $\hat{\mathbf{r}}=\mathbf{r} / r$. Then, the induced field reduces to

$$
\mathbf{E}^{\text {ind }} \underset{k r \rightarrow \infty}{\longrightarrow} \mathbf{f}(\Omega) \frac{e^{i k r}}{r},
$$

where $\Omega$ denotes the orientation of $\mathbf{r}$ and the far-field amplitude $\mathbf{f}=\tilde{\mathbf{f}}-(\tilde{\mathbf{f}} \cdot \hat{\mathbf{r}}) \hat{\mathbf{r}}$ is expressed in terms of the boundary current on the vacuum side as

$$
\tilde{\mathbf{f}}(\Omega)=i k \int_{S_{1}} d \mathbf{s} e^{-i k \hat{\mathbf{r}} \cdot \mathbf{s}} \mathbf{h}_{1}(\mathbf{s}) .
$$

Here we are relying on the fact that the contribution of $-\boldsymbol{\nabla} \phi$ to $\mathbf{E}^{\text {ind }}$ produces only longitudinal components. However, the far field is transvere and, consequently, entirely coming from the $i k \mathbf{A}$ contribution to $\mathbf{E}$. This gives rise to $\tilde{\mathbf{f}}$ [Eq. (50)], from which the longitudinal part has been subtracted to obtain $\mathbf{f}$.

The EIRE intensity can be obtained from the Poynting vector integrated over emission directions. The emitted energy per incoming electron is then given by

$$
\Delta E=\frac{c}{4 \pi} \int d t r^{2} \int d \Omega \hat{\mathbf{r}} \cdot[\mathbf{E}(\mathbf{r}, t) \times \mathbf{H}(\mathbf{r}, t)] .
$$

Now, expressing the fields in time Fourier transform [Eq. (1)] and using the far-field limit just discussed, we can decompose $\Delta E$ into photon-energy components $\hbar \omega$ as

\footnotetext{
${ }^{17} \mathrm{~A}$ word of caution should be added here: while EIRE is dominant at relatively high electron-beam energies (for example, above $10 \mathrm{keV}$ ), it decays and can be overcome by incoherent CL at sufficiently low electron energies.
}
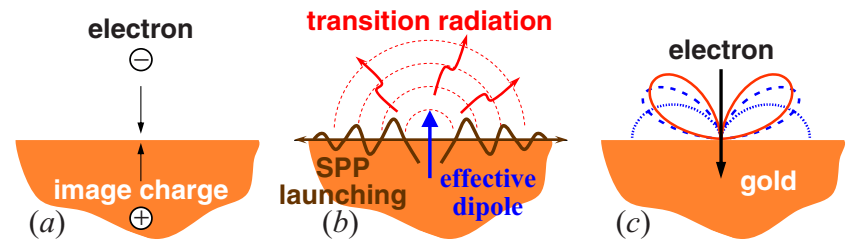

FIG. 22. (Color online) Intuitive explanation of transition radiation. (a) An electron cancels its image charge when it crosses a metal surface. (b) This cancellation creates an effective dipole capable of emitting light and generating plasmons. (c) The resulting transition radiation has a dipolar angular pattern (solid curve) similar to that arising from a dipole normal to the surface (dashed curve) and vanishing for grazing emission, in contrast to the pattern of the dipole in the absence of the surface (dotted curve). The angular pattern is calculated for $200 \mathrm{keV}$ electrons impinging on a planar Au surface and for $600 \mathrm{~nm}$ light wavelength.

$$
\Delta E=\int_{0}^{\infty} \hbar \omega d \omega \int d \Omega \Gamma_{\mathrm{CL}}(\Omega, \omega)
$$

where

$$
\Gamma_{\mathrm{CL}}(\Omega, \omega)=\frac{1}{4 \pi^{2} \hbar k}|\mathbf{f}(\Omega)|^{2}
$$

is the number of photons emitted per incoming electron, per unit of solid angle of emission $\Omega$, and per unit of photon frequency range $\omega$.

\section{Transition radiation}

When a fast charge impinges on a metal surface, the sudden annihilation of its image acts like an induced dipole that produces radiation, as shown in Fig. 22. This effect was predicted by Ginzburg and Frank (1946) and observed by Goldsmith and Jelley (1959) in the visible range for protons incident on $\mathrm{Ag}, \mathrm{Al}$, and $\mathrm{Au}$ surfaces. In fact, transition radiation occurs whenever a swift electron crosses the interface between different dielectric phases.

A detailed exposition of TR was given by TerMikaelian (1972), and a particularly useful result was produced by Kröger (1968), who reported a closed-form expression for the EELS probability of an electron traversing a thin film under arbitrary oblique incidence, including the contributions of Cherenkov modes and TR. This formula has been valuable in devising methods to eliminate retardation effects from measured spectra in the retrieval of bulk dielectric functions (Stöger-Pollach, 2008). In a related development, the theoretical analysis of the radiation produced by an electron passing by the center of a sphere showed an interesting evolution from CR (see Sec. IV.D) to TR when considering large or small spheres compared to the wavelength (Pogorzelski and Yeh, 1973).

On the experimental side, the results of Yamamoto, Sugiyama, and Toda (1996) and Yamamoto, Toda, and Araya (1996) for CR and TR in mica, Ag, Al, and Si films, including detailed comparison with theory, consti- 
tute an outstanding reference that illustrates the complex interplay between the two types of radiation emission channel in dielectric films as compared to metals (for example, $\mathrm{Ag}$ and $\mathrm{Al}$ show relatively featureless backward emission spectra for wavelengths above the bulk plasmon threshold in contrast to mica or $\mathrm{Si}$ ). In a separate work, Borziak et al. (1976) reported CL spectra for $5-500 \mathrm{eV}$ electrons impinging on $\mathrm{Ag}, \mathrm{Au}, \mathrm{Bi}, \mathrm{Cu}$, and $\mathrm{W}$ surfaces and showed a dense series of features that might originate in incoherent CL since coherent EIRE is strongly suppressed at such low energies (see Sec. IV.A.1). More recently, relativistic beams $(6.1 \mathrm{MeV})$ have been utilized to produce millimeter TR that is subsequently focused with a parabolic mirror (Naumenko et al., 2008).

The theory of TR for an electron normally incident on a planar surface is covered by several textbooks (Landau et al., 1984; Jackson, 1999). We outline it here in a form that is suitable for obtaining plasmon launching probabilities in Sec. V.D. We consider a swift electron moving along the $z$ axis with constant velocity vector $\mathbf{v}$ and crossing the free surface of a metal occupying the $z<0$ region (Fig. 9, right). ${ }^{18}$ The electric field produced by the moving charge in each medium $j$ (with $j=1$ and 2 representing the materials above and below the interface, respectively) can be separated into the contribution of the external electron field for an infinite bulk material plus the field reflected at the surface,

$$
\mathbf{E}=\mathbf{E}_{j}^{\mathrm{ext}}+\mathbf{E}_{j}^{\mathrm{ref}} .
$$

In particular, the electric field inside an infinite medium $j$ obtained from Eq. (2) after integrating the $z$ component of $\mathbf{q}$ reads

$$
\mathbf{E}_{j}^{\text {ext }}(\mathbf{r}, \omega)=\int \frac{d^{2} \mathbf{q}_{\|}}{(2 \pi)^{2}} \mathbf{E}_{j}^{\text {ext }}\left(\mathbf{q}_{\|}, z, \omega\right) e^{i \mathbf{q}_{\|} \mathbf{R}}
$$

with $^{19}$

$$
\mathbf{E}_{j}^{\mathrm{ext}}\left(\mathbf{q}_{\|}, z, \omega\right)=\frac{4 \pi i e}{|v| \epsilon_{j}} e^{i \omega z / v} \frac{\mathbf{q}-\mathbf{v} k \epsilon_{j} / c}{q^{2}-k^{2} \epsilon_{j}},
$$

where $\mathbf{q}=\left(\mathbf{q}_{\|}, \omega / v\right)$ and $\hat{\varphi}_{\mathbf{q}}=\hat{\mathbf{z}} \times \hat{\mathbf{q}}_{\|}$, so that the set $\left\{\hat{\mathbf{q}}_{\|}, \hat{\varphi}_{\mathbf{q}}, \hat{\mathbf{z}}\right\}$ forms a positively oriented three-dimensional (3D) reference frame. Faraday's law permits writing the corresponding magnetic field as

\footnotetext{
${ }^{18}$ The electron velocity varies dramatically inside the metal due to both heavy encounters with target atoms and inelastic collisions until it is eventually stopped. However, no relevant changes in the trajectory occur with significant probability for energetic electrons $(\geq 50 \mathrm{keV})$ within a distance of the order of the skin depth underneath the surface. Therefore, the constant velocity approximation is a reasonable one when describing TR produced by energetic electrons with the trajectory given by $x=y=0$ and $z=v t$.

${ }^{19}$ The remaining equations in this section apply to the situation depicted in Fig. 9 if we take $v<0$, but they are also valid for trajectories directed towards $z>0$ if we choose $v>0$ (i.e., $\mathbf{v}=v \hat{\mathbf{z}})$.
}

$$
\mathbf{H}_{j}^{\mathrm{ext}}\left(\mathbf{q}_{\|}, z, \omega\right)=\operatorname{sgn}(v) \frac{4 \pi i e q_{\|}}{c} e^{i \omega z / v} \frac{\hat{\varphi}_{\mathbf{q}}}{q^{2}-k^{2} \epsilon_{j}} .
$$

It should be stressed that the bulk fields are evanescent away from the trajectory unless $\epsilon_{j}$ is a positive real number and $v^{2} \epsilon_{j}>c^{2}$, in which case CR can be produced.

The reflected component of the field finds its sources in the induced surface charges and currents. The charges can be in turn expressed in terms of the currents by using the continuity equation. After some algebra, we obtain

$$
\begin{aligned}
& \mathbf{E}^{\mathrm{ref}}\left(\mathbf{q}_{\|}, z, \omega\right)
\end{aligned}
$$

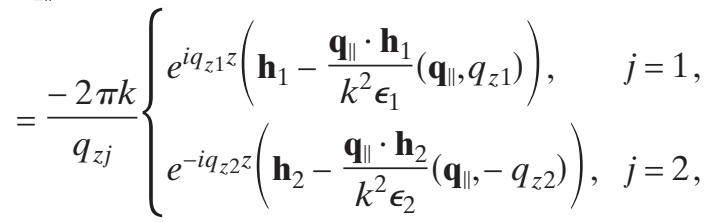

and

$$
\begin{aligned}
\mathbf{H}_{j}^{\mathrm{ref}}\left(\mathbf{q}_{\|}, z, \omega\right) & \\
= & \frac{-2 \pi}{q_{z j}} \begin{cases}e^{i q_{z 1} z}\left(\mathbf{q}_{\|}, q_{z 1}\right) \times \mathbf{h}_{1}, & j=1, \\
e^{-i q_{z 2} z}\left(\mathbf{q}_{\|},-q_{z 2}\right) \times \mathbf{h}_{2}, & j=2,\end{cases}
\end{aligned}
$$

where $q_{z j}=\sqrt{k^{2} \epsilon_{j}-q_{\|}^{2}}$. Here $\mathbf{h}_{j}$ are the noted surface currents (defined on either side of the interface), which are determined from the continuity of the parallel components of $\mathbf{E}$ and $\mathbf{H}$. These currents turn out to only have components along $\mathbf{q}_{\|}$, which allows us to write them as $\mathbf{h}_{j}=D \mu_{j} \hat{\mathbf{q}}_{\|}$. Finally, the reflected magnetic field reduces to

$$
\mathbf{H}_{j}^{\mathrm{ref}}\left(\mathbf{q}_{\|}, z, \omega\right)=-2 \pi e^{i q_{z j}|z|} D \mu_{j} \operatorname{sgn}(z) \hat{\varphi}_{\mathbf{q}},
$$

where

$$
\begin{aligned}
& \mu_{1}=\frac{1}{|v|}\left(\frac{\omega \epsilon_{2}+v q_{z 2} \epsilon_{1}}{q^{2}-k^{2} \epsilon_{1}}-\frac{\omega \epsilon_{1}+v q_{z 2} \epsilon_{1}}{q^{2}-k^{2} \epsilon_{2}}\right), \\
& \mu_{2}=\frac{-1}{|v|}\left(\frac{\omega \epsilon_{2}-v q_{z 1} \epsilon_{2}}{q^{2}-k^{2} \epsilon_{1}}-\frac{\omega \epsilon_{1}-v q_{z 1} \epsilon_{2}}{q^{2}-k^{2} \epsilon_{2}}\right),
\end{aligned}
$$

and

$$
D=\frac{2 i e q_{\|} / c}{q_{z 1} \epsilon_{2}+q_{z 2} \epsilon_{1}} .
$$

Performing the integral over the azimuthal angle of $\mathbf{q}_{\|}$, one finds

$$
\mathbf{H}_{j}^{\mathrm{ref}}(\mathbf{r}, \omega)=-i \operatorname{sgn}(z) \hat{\varphi} \int_{0}^{\infty} q_{\|} d q_{\|} D \mu_{j} e^{i q_{z j}|z|} J_{1}\left(q_{\|} R\right),
$$

where $\hat{\varphi}$ is the azimuthal unit vector at $\mathbf{r}$. The TR emission is obviously $p$ polarized for normal incidence, with the magnetic field pointing along the azimuthal direction, parallel to the surface. This is in agreement with experimental observations, in which residual s-polarized emission is claimed to originate in surface roughness (Hattendorff, 1977). 

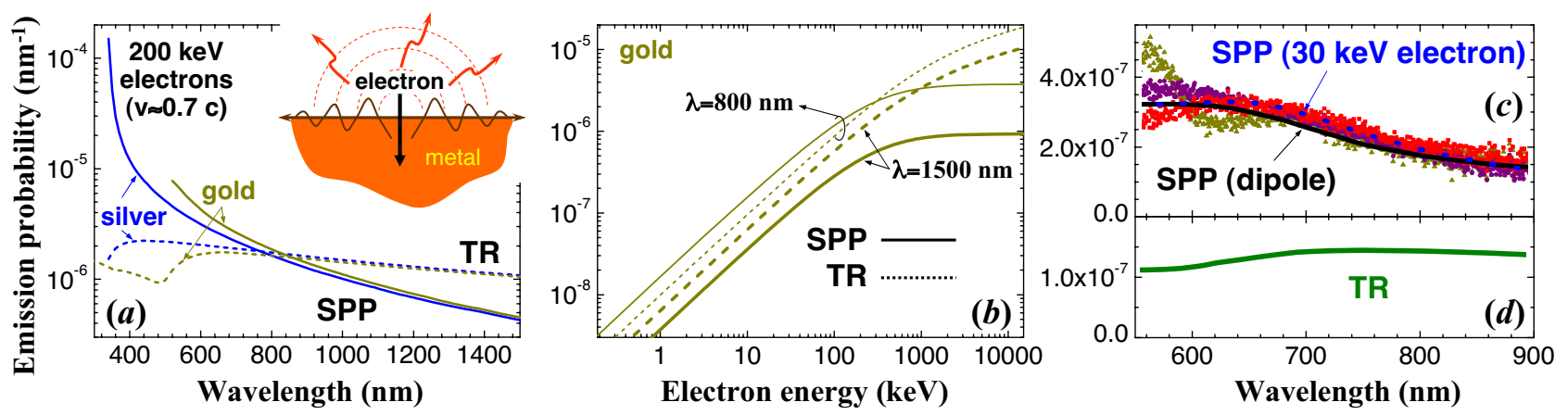

FIG. 23. (Color online) Excitation of SPPs and TR by electron bombardment of a metal surface. (a) Spectral dependence of the TR and SPP emission probabilities on electron beam bombardment under normal incidence. A $200 \mathrm{keV}$ electron impinges on a planar metal surface, giving rise to TR [dashed curves, calculated from Eq. (56)] and SPPs [solid curves, obtained from Eq. (71)]. The metal dielectric function has been taken from Johnson and Christy (1972). The probability is given per unit of light wavelength range. (b) Dependence of the TR and SPP emission probabilities in gold on electron kinetic energy for two different free-space light wavelengths, $\lambda=800$ and $1500 \mathrm{~nm}$. (c) Measured (symbols) and simulated (dashed curve) SPP emission probability spectra for normally incident $30 \mathrm{keV}$ electrons in gold. The SPP emission rate produced by an oscillating dipole placed at the surface is shown for comparison (solid curve), normalized to the spectral integral of TR and far-field dipole radiation. (d) Computed TR spectrum for $30 \mathrm{keV}$ electrons normally impinging on a gold surface. Notice that the probability is normalized per unit wavelength, so it corresponds to $(\omega / \lambda) \Gamma(\omega)$ with $\omega=2 \pi c / \lambda$. (c), (d) From Kuttge, Vesseur, Koenderink, et al., 2009.

The homogeneous field $\mathbf{H}_{j}^{\text {ext }}$ decays evanescently away from the electron trajectory (see Sec. II.A). Therefore, any TR emission must arise from $\mathbf{H}^{\text {ref }}$, which behaves as

$$
\mathbf{H}^{\mathrm{ref}}(\mathbf{r}, \omega) \underset{k r \rightarrow \infty}{\longrightarrow} \mathbf{f}_{H}(\theta, \omega) \frac{e^{i k r}}{r}
$$

in the far field of medium $1(z>0)$, taken to be vacuum. Asymptotic analysis of Eq. (55) shows that

$$
\mathbf{f}_{H}(\theta, \omega)=i k \cos \theta D \mu_{1} \hat{\varphi} .
$$

This expression must be evaluated at $q_{\|}=k \sin \theta$, where $\theta$ is the emission angle with respect to the surface normal. Finally, proceeding in a way similar to Sec. IV.B, the TR probability is given by the angular integral

$$
\Gamma_{\mathrm{TR}}(\omega)=\frac{1}{2 \pi \hbar k} \int_{0}^{\pi / 2} \sin \theta d \theta|\mathbf{f}(\theta, \omega)|^{2},
$$

performed over the upper hemisphere (vacuum).

Typical TR spectra are shown in Fig. 23(a) (broken curves) for $200 \mathrm{keV}$ electrons incident on $\mathrm{Ag}$ and $\mathrm{Au}$ surfaces. The spectral emission intensity exhibits an approximately linear decrease with wavelength above the SP threshold. The integrated emission in the $600-1200 \mathrm{~nm}$ region yields $\sim 10^{-3}$ photons per incident $200 \mathrm{keV}$ electron and shows a monotonic increase with probe energy [Fig. 23(b)].

\section{Cherenkov radiation}

The electric field set up by a swift electron in a homogeneous dielectric can exhibit far-field oscillatory behavior according to Eq. (5) whenever the charged probe moves faster than light in the medium under condition (7). This is reflected in the fact that the argument of the Bessel functions in Eq. (5) becomes imaginary under such conditions. Actually, these oscillations signal the emission of light, originally observed by Cherenkov (1934) and successfully explained soon after its discovery (Frank and Tamm, 1937; Tamm, 1939). The theory of the Cherenkov effect in homogeneous media was later generalized to accommodate frequency dispersion (Tamm, 1939; Fermi, 1940), and more sophisticated descriptions have been produced since then (Lalor and Wolf, 1971; Patro, 1982).

Cherenkov radiation has been extensively discussed [see Ginzburg (1996), and references therein], but a particularly interesting demonstration was reported by Ohkuma et al. (1991), who observed the effect for bunched ultrarelativistic electrons moving faster than light in air.

The probability of CR emission in a homogeneous material was first obtained by Frank and Tamm (1937). It can be easily derived by integrating Eq. (20) over wave-vector transfers, assuming real and local $\epsilon(\omega)$. One finds

$$
\Gamma_{\text {Che }}(\omega)=\frac{e^{2} L}{\hbar}\left(\frac{1}{c^{2}}-\frac{1}{v^{2} \epsilon(\omega)}\right),
$$

where the dependence on emission frequency comes exclusively from the dielectric function. Equation (57) is a pillar in the design of Cherenkov detectors for highenergy physics, in which CR signals the passage of charged particles resulting from energetic subatomic collisions (Julley, 1958).

Nontrivial finite-trajectory effects have been successfully described using the field of Eq. (4) in structures of large dimensions compared to the emission wavelength, for which the contribution of reflection at the boundaries can be neglected (Zrelov and Ružička, 1989; Ružička and Zrelov, 1993; Ciljak et al., 2003). However, this approximation is not sufficient for small samples like the thin films theoretically addressed by Kröger (1968). Actually, the experiments reported by Yamamoto, Sug- 


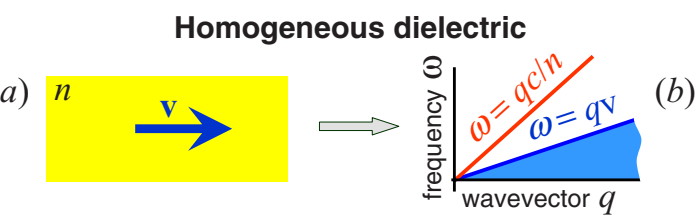

1D photonic crystal

(c)

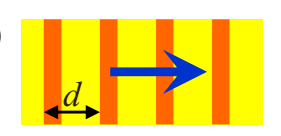

(e)

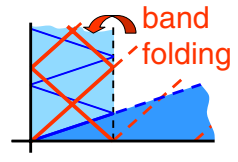

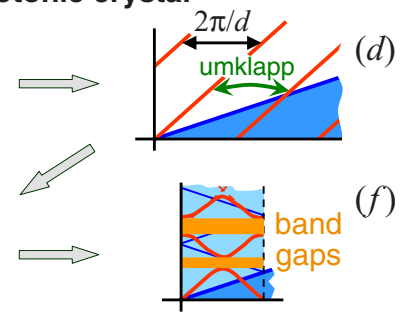

FIG. 24. (Color online) Tutorial description of the Cherenkov effect in a photonic crystal. A charge moving in a homogeneous dielectric with velocity below the Cherenkov threshold $v<c / n$ (a) cannot transfer energy and momentum [shaded region in (b)] overlapping the light dispersion line [upper line in (b)]. However, a periodic modulation of the index of refraction (c) allows light to undergo umklapp processes [i.e., absorption or emission of lattice momentum, as shown in (d)], so that band folding over the first Brillouin zone of the resulting photonic crystal (e) produces mixing and gap openings in the photonic band structure (f). The charge can now couple to propagating optical modes.

iyama, and Toda (1996) on mica and Si films show a complex evolution of the CR intensity with film thickness (particularly for backward emission) that is correctly explained by Kröger's formula.

The Cherenkov effect has been theoretically investigated for a charge traversing planar interfaces (Kröger, 1968; Von Festenberg and Kröger, 1968; Lucas and Kartheuser, 1970; Ter-Mikaelian, 1972) or moving parallel to a surface (Schieber and Schächter, 1998), for layered structures (Bolton and Chen, 1995; Lastdrager et al., 2000), for an electron moving along the axis of a cylinder (Olsen and Kolbenstvedt, 1980), and for beams passing near a number of different geometries (García de Abajo et al., 2004). Other related effects have been explored, such as CR due to bunched electrons moving along waveguides (Onishchenko et al., 2002), solitons producing CR in optical fibers (Akhmediev and Karlsson, 1995; Skryabin et al., 2003), a second narrower Cherenkov cone in strongly dispersive active media (Carusotto et al., 2001), terahertz SPPs emitted by browsing a light spot at superluminal velocities on a planar surface (Bakunov et al., 2005), and inverted Cherenkov cones in materials of negative refractive index (Veselago, 1968; Matloob and Ghaffari, 2004; Averkov and Yakovenko, 2005; Xi et al., 2009).

Furthermore, exotic production of $\mathrm{CR}$ has been observed due to superluminal vortices in Josephson junctions (Goldobin et al., 1998), whereas subluminal CR emission has been reported assisted by phonons that supply the required extra momentum (Stevens et al., 2001).

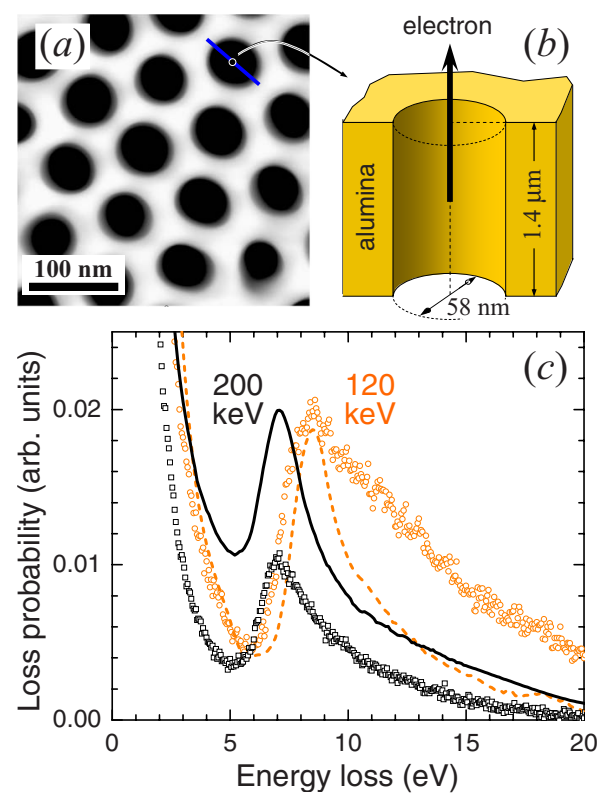

FIG. 25. (Color online) Experimental observation of CR losses in a photonic crystal. A 1.4- $\mu \mathrm{m}$-thick self-standing porous alumina film, as shown in the TEM image of (a), is used to measure the energy loss experienced by electrons moving inside one of the holes (b). The measured energy-loss spectrum is represented in (c) for 120 and $200 \mathrm{keV}$ electrons (symbols) alongside theoretical calculations based on full solution of Maxwell's equations for an electron moving inside the $2 \mathrm{D}$ photonic crystal (dashed and solid curves, respectively). The theory has been convoluted with a measured ZLP of $0.86 \mathrm{eV}$ FWHM. Adapted from García de Abajo et al., 2003.

\section{Cherenkov effect in photonic crystals}

An interesting situation is presented when an electron moves inside a photonic crystal, consisting of a periodic modulation of the index of refraction along certain spatial direction(s). The resulting $\mathrm{CR}$ is then affected by the photonic band structure, which can considerably differ from the vacuum light cone, as schematically shown in Fig. 24 for a 1D crystal. The loss of translational invariance allows subluminal electrons to couple to photonic bands of the crystals, thus resulting in the emission of CR.

This effect has been theoretically analyzed by Luo et al. (2003) and Kremers et al. (2009) for electrons moving perpendicularly with respect to the pores of a $2 \mathrm{D}$ photonic crystal. They found a complex evolution of the electric field, which is molded by the photonic bands.

An experimental investigation of the interaction of fast electrons with a 2D photonic crystal has been reported (García de Abajo et al., 2003) and is shown in Fig. 25 . The photonic crystal consisted of a porous alumina film, grown by electrochemical methods under the appropriate conditions to form a locally regular array of pores (Masuda and Fukuda, 1995). The precise control of electron optics in a STEM allows focusing of the probe so that it passes along one of the pores of the crystal in aloof configuration, with the beam positioned tens of nanometers away from the pore wall. The result- 


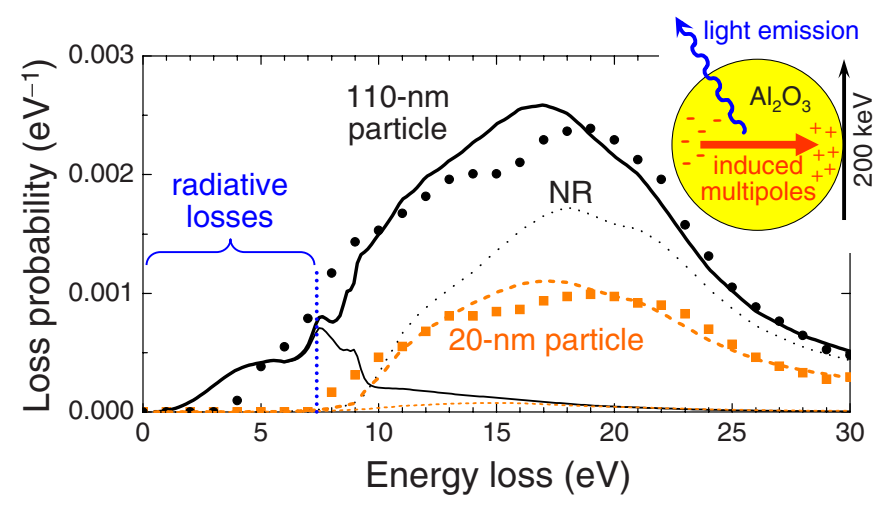

FIG. 26. (Color online) Losses in aloof trajectories due to diffraction radiation. Measured and simulated energy-loss spectra shown for $200 \mathrm{keV}$ electrons passing just outside alumina spherical particles. Symbols: Measured data taken from Abe et al., 2000. Thick curves: Retarded EELS calculations using Eq. (33). Dotted curves: Nonretarded EELS calculation obtained from Eq. (31). Thin solid curve: CL calculation for the larger sphere using Eq. (58). The computed probability is given per $\mathrm{eV}$ of energy-loss range. The dielectric function of alumina is taken from Palik, 1985.

ing loss spectra display prominent peaks around 7-9 eV, depending on the electron energy, which were attributed to radiative losses produced by coupling to photonic modes of the crystal. The spectral features are of course related to the photonic LDOS, as discussed in Sec. III.H, and they exhibit singularities similar to those explained by Van Hove (1953), but now arising from characteristic points in photonic rather than electronic bands. These results are indirect evidence of CR in photonic crystals. A direct experimental detection of CL in these systems has not yet been reported.

Incidentally, similar effects could be responsible for unusual spectral features in porous silicon at large beam-surface separation (Williams et al., 2000).

\section{E. Diffraction radiation}

We have seen that radiation can be produced when an electron crosses a dielectric interface or when it moves faster than light in a homogeneous medium. Another common source of light emission originates in the passage of the electron near a structured surface (without actually crossing it), giving rise to the so-called diffraction radiation (Potylitsyn, 1998). For instance, this effect occurs if a swift charge passes near and perpendicularly to metallic slits, a geometry that has been actually used for beam diagnosis in electron accelerators (Karataev et al., 2004). Incidentally, diffraction radiation can be a significant source of electron decoherence (García de Abajo, 2009).

We present in Fig. 26 the effect of diffraction radiation on the loss spectrum of electrons grazingly passing with respect to alumina spheres of different sizes. The largest sphere produces a sizable inelastic signal in the region below $\sim 7 \mathrm{eV}$, in which the material is basically transparent for the dimensions under consideration. This actu- ally originates in the polarization charges induced by the passing electron in the sphere, which oscillate, giving rise to light emission. Understandably, the smaller sphere is poorly polarized and, therefore, couples weakly to propagating light, so that it does not produce observable losses within the low-energy end of the spectrum.

The emission probability admits a closed-form expression similar to Eq. (33) (García de Abajo, 1999c),

$$
\begin{aligned}
\Gamma_{\mathrm{CL}, \mathrm{sph}}(\omega)= & \frac{e^{2}}{c \hbar \omega} \sum_{l=1}^{\infty} \sum_{m=-l}^{l} K_{m}^{2}\left(\frac{\omega b}{v \gamma}\right) \\
& \times\left(C_{l m}^{M}\left|t_{l}^{M}\right|^{2}+C_{l m}^{E}\left|t_{l}^{E}\right|^{2}\right) .
\end{aligned}
$$

One can easily see from Eqs. (C1) and (C2) that the scattering coefficients satisfy the relation $\left|t_{l}^{\nu}\right|^{2}=\operatorname{Im}\left\{t_{l}^{\nu}\right\}$ in spheres made of real dielectric function, implying that the loss probability equals the CL rate in that case, because the material is unable to absorb any energy [cf. Eqs. (33) and (58)]. The CL rate calculated from Eq. (58) coincides with the loss probability in the region below $7 \mathrm{eV}$ of Fig. 26 (thin and thick continuous curves, respectively), which further supports the dominant effect of the diffraction radiation mechanism in that part of the spectrum.

Incidentally, the EELS spectra of Fig. 26 show absolute maxima in the $15-20 \mathrm{eV}$ range, corresponding to plasmons of the alumina spheres (for comparison, the bulk plasmon occurs at $25 \mathrm{eV}$ and the planar SP at $21 \mathrm{eV})$.

\section{Smith-Purcell emission}

The effect discovered by Smith and Purcell (S-P) (1953) is a well-known example of diffraction radiation. They recorded light emitted when an electron beam passes parallel to and above a metallic grating. The direction and wavelength of emission followed a simple relation derived from a Huygens construction. More precisely,

$$
\Delta t=(d / c) \cos \theta=d / v+\lambda n / c,
$$

where $n$ is an integer number, $\lambda$ is the wavelength, and the rest of the parameters are defined in the following diagram:

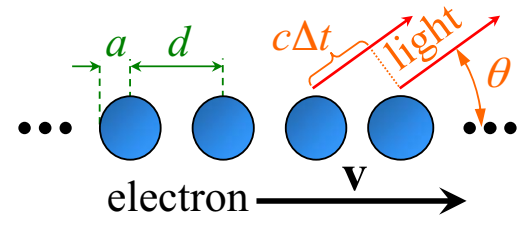

Equation (59) arises from the condition of constructive interference in the emission originating in contiguous spheres.

Successive experimental demonstrations of this effect were carried out using 30-300 keV electrons to produce light of wavelength lying in the micron region (Salisbury, 1970; Bachheimer, 1972). Also, relativistic beams have been employed for generating millimeter radiation 
(Doucas et al., 1992; Shibata et al., 1998; Blackmore et al., 2008) which turns out to be very intense along the forward direction (Woods et al., 1995). Furthermore, $20-40 \mathrm{keV}$ dense electron beams coming from conventional electron microscopes and coupled to metallic gratings of periods in the submillimeter domain have been used to produce supergeneration of far-infrared radiation (Temkin 1998; Urata et al., 1998). ${ }^{20}$ In this context, electrons passing along periodically corrugated nanoholes have been recently shown to produce light with a substantial degree of tunability (Adamo et al., 2009).

Early theoretical analyses of the S-P effect relied on diffraction of the evanescent waves accompanying the moving electron (Toraldo di Francia, 1960; van den Berg, 1973; van den Berg and Tan, 1974; Haeberlé et al., 1994). It was also shown that an equivalent description results from considering the currents induced by the passage of the electron on the grating surface (Brownell et al., 1998). Moreover, a large deal of work has been devoted to studying the conditions upon which this effect could be utilized for efficient $\mathrm{x}$-ray generation (Moran, 1992), for instance, in free-electron lasers ( $\mathrm{Li}$, Imasaki, Gao, et al., 2007; Li, Imasaki, Yang, et al., 2007; Liu et al., 2007).

The excellent spatial resolution of electron microscopes could be used to direct a beam parallel to an array of nanoparticles and produce S-P emission. This situation is considered in Fig. 27, which predicts intense supercontinuum emission in the uv domain. This type of submicron light emitters could find eventual application in integrated photonic devices. Interestingly, Fig. 27 shows the presence of an absolute threshold below which no emission is possible because Eq. (59) cannot be satisfied.

In recent works, the S-P emission from photonic crystals has been extensively investigated (Pendry and Martín-Moreno, 1994; García de Abajo and Blanco, 2003; Ochiai and Ohtaka, 2004a, 2004b, 2005) and compared with gratings (Ohtaka and Yamaguti, 2001; Yamaguti et al., 2002) using theoretical tools. Besides, experimental observations have been reported for millimeter waves emitted by electrons passing near photoniccrystal opals (Yamamoto et al., 2004). Finally, interesting effects due to finite size of the periodic structure along the direction of the beam have been noted (Ochiai and Ohtaka, 2006) and experimentally observed (Horiuchi et al., 2006).

\footnotetext{
${ }^{20}$ Within linear response theory, valid for most situations considered in this review, the EELS and CL probabilities are proportional to the square of the particle charge, and they scale linearly with the beam intensity for moderate currents. However, bunching can produce nonlinear scaling when the electrons move closer to each other than the emission wavelength (Urata et al., 1998), so that they behave like a composite charge, the square of which is obviously larger than the sum of the squares. This leads to super-radiant Smith-Purcell emission, with $N$ bunched electrons emitting in proportion to $N^{2}$.
}

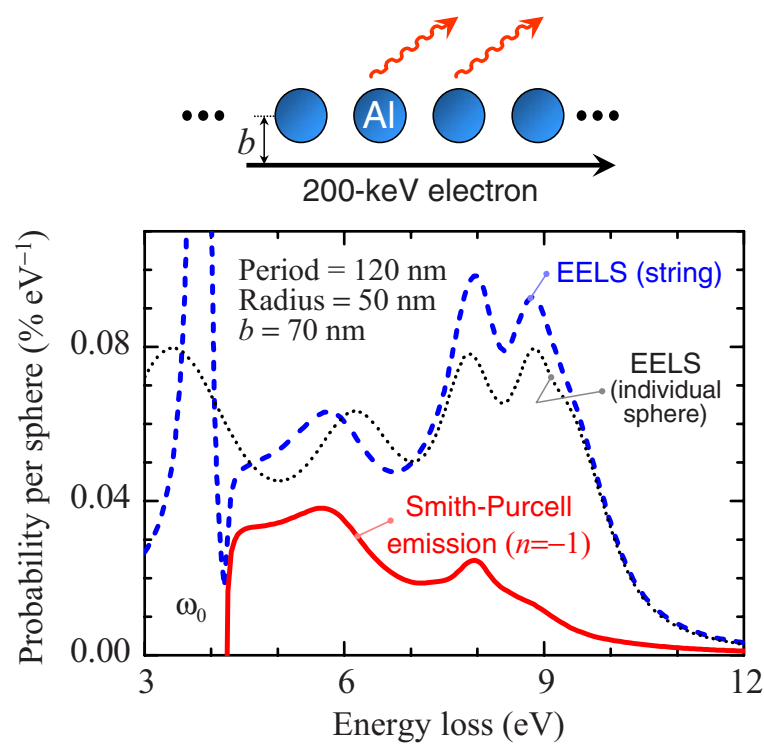

FIG. 27. (Color online) Smith-Purcell effect in particle chains. Energy-loss probability and Smith-Purcell radiation emission for an electron moving parallel to an infinite periodic string of aligned Al nanoparticles (see upper inset). The probability is normalized per sphere and per eV of energy-loss range. The loss probability for an individual sphere is also shown for comparison. Adapted from García de Abajo, 1999a.

\section{F. Cathodoluminescence and plasmons}

Plasmons in metallic nanoparticles have finite lifetime due to radiative decay, Landau damping ( $e-h$ pair creation), and other absorption mechanisms. The former is rather efficient when the particle has non-negligible size compared to the corresponding light wavelength, as discussed below. Actually, the CL signal resulting from plasmon decay offers an opportunity to resolve plasmons spectrally and spatially.

\section{Plasmons in metallic films and gratings}

An electron traversing a thin film can give rise to a peak in the CL emission at the bulk plasmon frequency $\omega_{p}$, as predicted by Ferrell (1958), who found that the emitted light was $p$ polarized (magnetic field parallel to the film) for normal incidence, and the emission intensity vanished at normal and grazing directions for a Drude metal. This prediction was soon confirmed experimentally by Steinmann (1960) and Brown et al. (1960) in Ag films and later by Arakawa et al. (1964) in $\mathrm{Ag}$ and $\mathrm{Mg}$ films. A study by Ritchie and Eldridge (1962) generalized Ferrell's results to arbitrary metals and $v \ll c$, while the comprehensive work of Cram and Arakawa (1967) showed good agreement between theoretical and experimental results in Ag films compared to the same absolute-intensity scale. Additionally, the Ferrell mode was observed using optical excitation rather than electron beams (Brambring and Raether, 1965), and its presence was shown to affect the response properties of thin films near the bulk plasmon frequency (Kliewer and Fuchs, 1967). Subsequent TEM experi- 

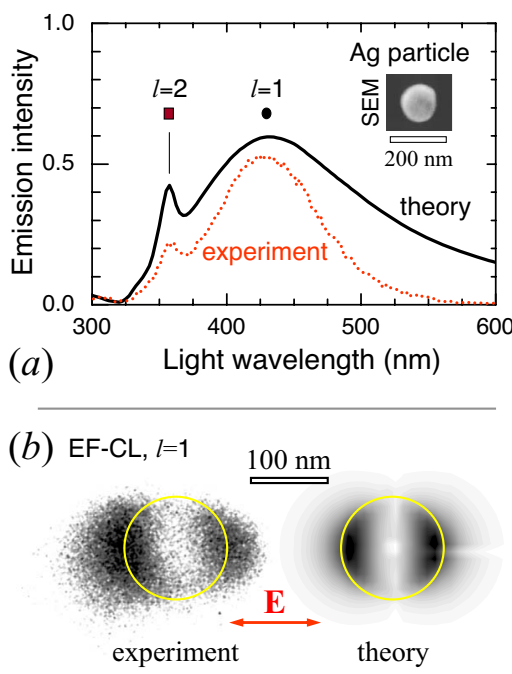

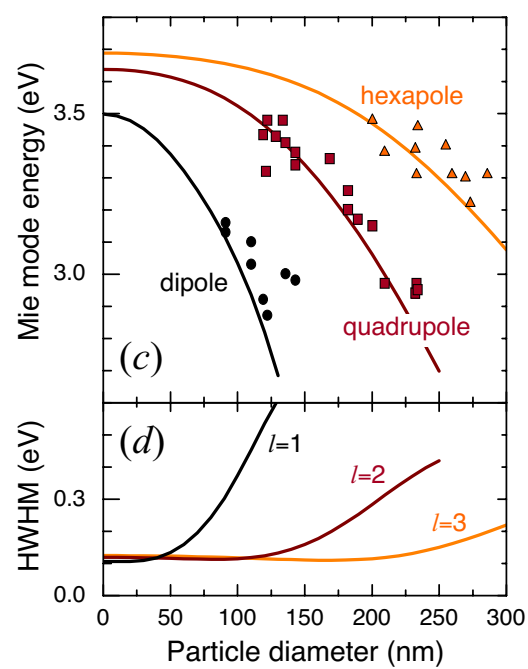

FIG. 28. (Color online) Plasmon mapping of single metallic nanoparticles using CL. (a) Measured and calculated CL spectra for a $122 \mathrm{~nm}$ Ag nanoparticle under grazing incidence, showing dipole $(l=1)$ and quadrupole $(l=2)$ plasmons. The dielectric function of $\mathrm{Ag}$ is taken from Johnson and Christy (1972). (b) Energy-filtered CL scan corresponding to the dipole-mode maximum of the spectra shown in (a). The collected light is passing through a polarizer with the orientation shown by the arrow. (c) Symbols: Measured dispersion of multipole plasmons with particle size. Curves: Mie theory. (d) Plasmon width obtained from Mie theory. Adapted from Yamamoto, Araya, and García de Abajo, 2001. ments managed to resolve the dispersion relation of the Ferrell mode in Al (Vincent and Silcox, 1973), basically following the transversal bulk plasmon curve of Fig. 8(b).

In contrast, SPPs in a thin film cannot directly couple to external light because their parallel momentum lies outside the light cone, according to Eq. (27), with $\epsilon_{1}$ and $\epsilon_{2}$ having opposite signs. However, SPPs can be made to radiate if they find obstacles in their propagation along an otherwise planar surface. In particular, gratings provide a source of parallel momentum that plasmons can use to couple to external light. The optical emission resulting from excitation of SPPs by electrons impinging on a grating was first observed by Teng and Stern (1967). They managed to correlate SPP-related peaks in the angular distribution of the emission with dips observed in the specular reflectivity of periodically corrugated surfaces.

Heitmann (1977) used CL to map the SP dispersion in sinusoidal Ag gratings of different heights. The gratings assisted SPPs to couple to freely propagating light. His analysis of extrapolated results in the limit of shallow corrugation was in excellent accord with both the dispersion relation and the momentum-energy width predicted by Eq. (27). Furthermore, his results for stronger modulation of the grating profile resulted in dispersion relations further apart from the light line, so that the grating contributed to increase the binding of SPPs to the surface. A similar increase in surface-mode binding driven by periodic corrugations has also been observed in microwaves trapped by drilled metal films (Ulrich and Tacke, 1973). This behavior is accompanied by gaps in the boundaries of the Brillouin zones, as first discussed by Ritchie et al. (1968), for $p$-polarized light incident on gratings and later studied in 2D plasmon crystals (Kitson et al., 1996), capable of guiding plasmons through lines of defects (Bozhevolnyi et al., 2001).

Two recent outstanding experiments have independently supplemented Heitmann's work by out-coupling electron-generated SPPs in planar surfaces using distant gratings (Bashevoy et al., 2006; van Wijngaarden et al., 2006) thus suggesting the possibility of using fast elec- trons as plasmon sources. We address this issue in more detail in Sec. V.D.

\section{Plasmon mapping}

Cathodoluminescence has been used to map plasmon excitations in scanning mode by analogy to the EELS plasmon-mapping study presented in Fig. 16. The CL signal is generally much weaker than the loss intensity, which is problematic when good signal-to-noise ratio is needed to resolve neighboring spectral features. However, CL has the advantage that it can be performed in SEMs, which are more widely available than TEMs, and it works for thick samples since there is no need to collect transmitted electrons.

Several examples of application of this technique to spatially resolve plasmons in nanostructures have been presented over the last few years, including studies of plasmons in spherical Ag nanoparticles (Yamamoto, Araya, and García de Abajo, 2001), gratings (Yamamoto, Araya, Toda, and Sugiyama, 2001; Bashevoy et al., 2007), holes in thin films (Degiron et al., 2004), particle dimers and nanorods (Yamamoto, Nakano, and Suzuki, 2006; Vesseur et al., 2007), silver nanotriangles (Chaturvedi et al., 2009), Fabry-Perot resonators for SPPs in gold (Kuttge, Vesseur, and Polman, 2009), and whispering gallery modes in ringlike grooves (Vesseur $e t$ al., 2009).

A characteristic example of these studies is shown in Fig. 28 for Ag particles. The spectra of Fig. 28(a) unveil the excitation of a broad dipole mode and a narrower quadrupole. The measurement is in good agreement with the theory of Eq. (58). By scanning the electron beam over the particle and collecting polarized photons emitted at the peak wavelength, one can form the image of the dipole plasmon shown in Fig. 28(b). The intensity of each pixel represents the photon rate measured when the electron is positioned at that spot of the sample. This plasmon map displays two characteristic maxima, one on either side of the particle, along the direction of the polarizer. This study was carried out for particles of various sizes, the results of which are summarized in Fig. 28(c). 


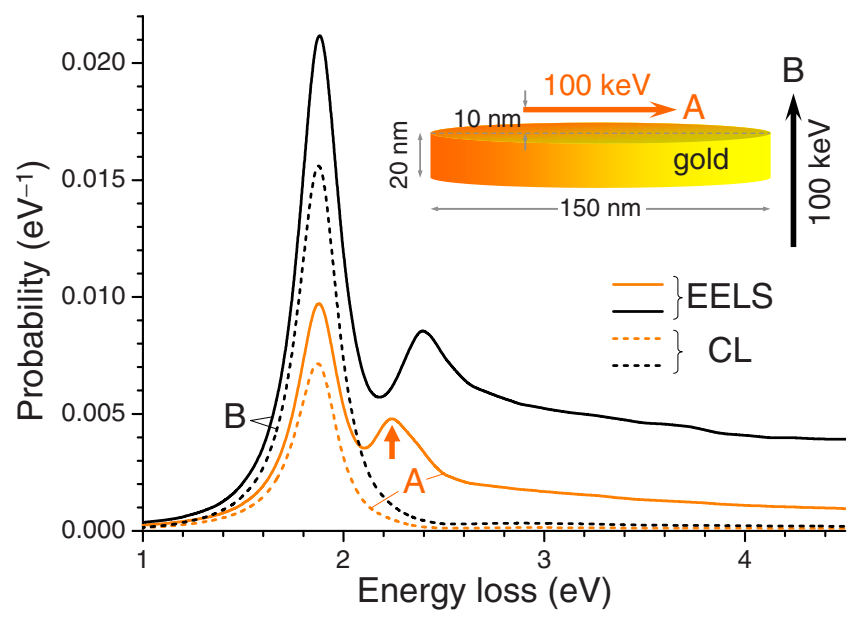

FIG. 29. (Color online) Comparison of EELS and CL intensities in a gold nanodisk. Two different electron beam trajectories are considered (see inset) separated $10 \mathrm{~nm}$ from the disk in both cases. The disk edges have been rounded with a curvature radius of $3 \mathrm{~nm}$. These results have been obtained using the retarded BEM with the dielectric function of gold from Johnson and Christy, 1972.

The plasmon modes show a distinctive redshift with increasing particle size, in good agreement with the theory of Mie (1908), who provided the basis for understanding light scattering by spherical particles. The origin of these shifts is retardation, as discussed in Sec. III.D.2, and they occur when the diameter of the particle is a sizable fraction of the wavelength. As an empirical rule, we can assert that retardation effects show up for radius $a$ $z \lambda l / 2 \pi .^{21}$ These effects are accompanied by an increase in the width of the multipole plasmons with particle size, which arises as a result of stronger coupling to propagating light [see Fig. 28(d)] (Myroshnychenko, RodríguezFernández, et al. 2008).

Figures 28(a) and 28(b) demonstrate that aloof geometries (external trajectories) provide significant information on the particle plasmons. This idea is further shown in Fig. 29, which presents a quantitative comparison of EELS and CL probabilities for gold disks. The difference between these two probabilities corresponds to events in which the lost energy is absorbed by the particle rather than being reemitted. These calculations have been carried out using the boundary element method described in Sec. III.E.3.

The CL spectra of Fig. 29 (dashed curves) show just a subset of the features observed in the loss spectra (solid curves), as an indication that some plasmon excitations are dark modes, in the sense that their main decay mechanism is via absorption by the particle rather than

\footnotetext{
${ }^{21}$ The multipolar order $l$ intervenes in determination of the relevant distance to which the wavelength has to be compared for assessing the relative importance of retardation. This must be of the order of the separation between nodes in the induced surface charge, which is $\sim a / l$, since the interaction between charges at larger distances is partially washed out by cancellation of contributions with different signs.
}

coupling to radiation (Chu et al., 2009; Koh et al., 2009). More precisely, the feature marked by an arrow in the loss spectrum of trajectory $\mathrm{A}$ is not found in the light cross section of randomly oriented disks (not shown), but it can be clearly resolved by EELS.

The spatial resolution of CL should be analogous to EELS for mapping plasmons, as shown in Fig. 14(b). This figure proves that aloof beams can yield similar emission intensities as penetrating electrons, in agreement with the images of Fig. 28(b). Finally, an additional thought on the aloof configuration: the incoherent CL signal, which we argued in Sec. IV.A.2 to be small compared to the coherent EIRE contribution reported in these figures, should actually be smaller when the electron does not traverse the metal and cannot create localized excitations, which are the main source of incoherent CL.

\section{G. Ultrafast cathodoluminescence}

In a recent development, the $\mathrm{CL}$ technique has evolved to allow picosecond resolution using a microscope in which the electron source consists of a photocathode excited by external light pulses, so that the time-resolved CL emission can be assigned an origin of excitation time determined for each of the pulses, taking into account the time of flight delay of the electron in its way to the target. This technique has recently been utilized to resolve charge-carrier dynamics in quantum dots and quantum wells with $50 \mathrm{~nm}$ spatial resolution and 10 ps time resolution (Merano et al., 2005; Sonderegger et al., 2006). Similarly, picosecond electron pulses have been used to develop ultrafast electron microscopy (Lobastov et al., 2005), so far applied for simultaneous dynamical and structural characterization (Grinolds et al., 2006; Barwick et al., 2008). These studies open an avenue toward a whole range of interesting phenomena in the domain of the ultrafast nanoworld.

\section{RELATED PHENOMENA AND SUGGESTED EXPERIMENTS}

Electron microscopy is a rather versatile discipline that finds its natural domain in the nanoworld. We can certainly think of many new phenomena to be explored with focused electron beams, particularly when lowenergy valence excitations are involved. In this section, we go through some of them.

\section{A. Mechanical momentum transfer}

A fast electron beam has the potential to act as a nanotool that manipulates molecules and nanoparticles by transferring linear and angular momenta. This should become a good complement to optical tweezers, currently employed to trap small particles ranging in size from nanometers to several microns (Meiners and Quake, 2000) and to study their mechanical behavior by exerting electromagnetic forces (Korda et al., 2002; Grier, 2003). Studies based on optical tweezers range 
from elastic properties of deformable living cells (Guck et al., 2000) to fluctuation forces in biomolecules (Meiners and Quake, 2000). Likewise, an electron microscope can modify (and simultaneously image) small structures with far greater resolution than is achieved by optical imaging. A more sophisticated setup would involve in vacuo optical tweezers integrated in an electron microscope for observation of trapped particles. This system is experimentally challenging because it requires the trapping of particles in vacuum, for which pioneering results on optical levitation were reported three decades ago by Ashkin and Dziedzic (1976). A solution that is perhaps more compatible with trapping in vacuum consists in relying on evanescent fields (e.g., localized SPs), as proposed by Novotny et al. (1997) and recently demonstrated by Righini et al. (2008).

Here we estimate the magnitude of the momentum transfer imparted on small objects using the methods elaborated in previous sections (García de Abajo, 2004). The electromagnetic force is obtained by integrating Maxwell's stress tensor over a surface surrounding the object (Jackson, 1999). Performing the time integral of this force, we find

$$
\text { momentum transfer }=\int \mathbf{F}(t) d t=\int_{0}^{\infty} \mathbf{F}(\omega) d \omega,
$$

where the spectral decomposition in the last identity is similar to the one used for the EELS probability in Sec. II.B.

For simplicity, we first limit our discussion to a small isotropic particle described by its electric polarizability $\alpha(\omega)$. The force exerted by an external field $\mathbf{E}(\mathbf{r}, \omega)$ acting on such a particle is given by (Gordon and Ashkin, 1980)

$$
\mathbf{F}(\omega)=\frac{1}{\pi} \operatorname{Re}\left\{\alpha(\omega) \sum_{j} E_{j}(\mathbf{r}, \omega) \nabla\left[E_{j}(\mathbf{r}, \omega)\right]^{*}\right\},
$$

where the sum runs over Cartesian components and the external field supplied by the electron is given by Eq. (4). Using this in Eq. (61), one finds

$$
\begin{aligned}
\mathbf{F}(\omega)= & \frac{2 e^{2} \omega^{3}}{\pi v^{5} \gamma^{3}}\left[-\operatorname{Re}\{\alpha\} f^{\prime}\left(\frac{\omega b}{v \gamma}\right) \hat{\mathbf{x}}\right. \\
& \left.+2 \gamma \operatorname{Im}\{\alpha\} f\left(\frac{\omega b}{v \gamma}\right) \hat{\mathbf{z}}\right],
\end{aligned}
$$

where the function $f$ is the same as in Eq. (35) and the particle is located at a distance $b$ from the electron beam along the negative $x$ axis.

Symmetry considerations lead to the conclusion that Rayleigh scattering by the particle produces a radiation pattern symmetric with respect to the $x-y$ plane. This means that the overall transfer of momentum in the form of induced radiation is zero in the small-particle limit, so that Eq. (60) accounts for all momentum transfer emanating from the moving electron along the $z$ axis parallel to the trajectory. Then, the contribution of each $\omega$ component to the electron energy-loss probability is $v F_{z}(\omega)$. In fact, we find from Eqs. (62) and (34) the iden-

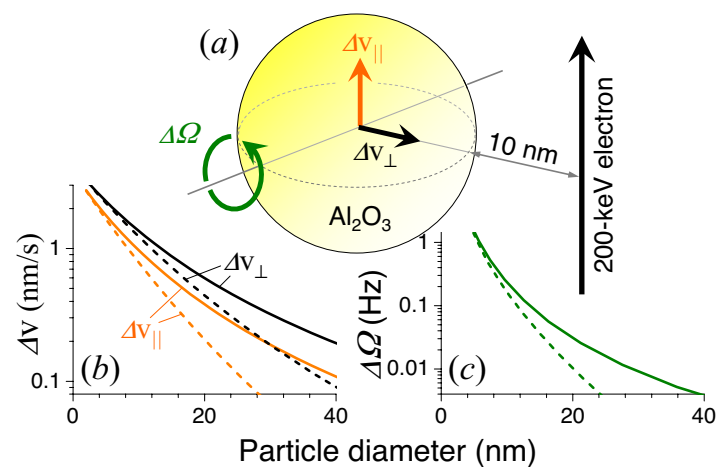

FIG. 30. (Color online) Momentum transfer from an electron to a spherical alumina particle. (a) Sketch of a $200 \mathrm{keV}$ electron passing at a distance of $10 \mathrm{~nm}$ from the surface of an alumina particle. (b) Linear-momentum transfer, quantified by the velocity change of the particle along directions parallel and perpendicular to the beam and normalized per incident electron. Solid curves: Full electromagnetic calculation. Dashed curves: Dipole approximation [Eq. (62), with the prescription of Eq. (36) for $\alpha$ ]. (c) Change in rotational velocity produced by angular momentum transfer from a single electron: Full electromagnetic simulation (solid curve) compared to the dipole approximation [dashed curve, Eq. (63)]. From García de Abajo, 2004.

tity $v F_{z}(\omega)=\hbar \omega \Gamma_{\mathrm{EELS} \text {,dip }}(\omega)$ : it is reassuring that the mechanical energy exchanged through momentum transfer equals the loss probability multiplied by the transferred energy for small targets.

The mechanical effect can be significant in nanoparticles, as shown in Fig. 30(b), which compares Eq. (62) (dashed curves) with the results obtained when all multipoles are included (solid curves). For instance, a typical STEM beam with a current of $1 \mathrm{nA}$ passing $10 \mathrm{~nm}$ away from the surface of a $40 \mathrm{~nm}$ alumina particle exerts a macroscopic attractive acceleration equal to 0.38 times that of the gravity.

The transfer of angular momentum $\Delta \mathcal{L}$ can be written

$$
\Delta \mathcal{L}=\int_{0}^{\infty} \tau(\omega) d \omega
$$

where the torque $\tau$ is also given by the integral of the Maxwell stress tensor (Jackson, 1999) over a surface surrounding the target. For the small particle considered above, one obtains

$$
\tau(\omega)=\frac{16 e^{2} \omega^{2}}{\pi v^{4} \gamma^{3}} K_{0}\left(\frac{\omega b}{v \gamma}\right) K_{1}\left(\frac{\omega b}{v \gamma}\right)\left[\operatorname{Im}\{\alpha\}-\frac{2 k^{3}}{3}|\alpha|^{2}\right] .
$$

Interestingly, the expression inside the square brackets of this equation vanishes for a nondissipative particle. ${ }^{22}$ Equation (63) shows that the torque produced on a small particle drops exponentially with impact parameter [see

\footnotetext{
${ }^{22}$ This is a consequence of the optical theorem for small particles, stating that $\operatorname{Im}\{-1 / \alpha\} \geqslant 2 k^{3} / 3$, with the equality occurring only for nonabsorbing particles (van de Hulst, 1981).
} 
Fig. 30(c)], but it is still sufficient to cause an astonishing $23 \mathrm{MHz} / \mathrm{s}$ angular acceleration when a $1 \mathrm{nA}$ electron beam passes $10 \mathrm{~nm}$ away from a $40 \mathrm{~nm}$ alumina particle. The type of experiment needed to demonstrate this prediction should be attainable using the combination of optical tweezers and TEM suggested above. One may want to see what happens to the particle as it spins increasingly faster until centrifugal forces compete with atomic binding.

\section{B. Vicinage effects}

At half the speed of light, electrons in a typical $100 \mathrm{keV}, 0.1 \mathrm{nA}$ STEM beam are separated by an average distance of $26 \mathrm{~cm}$ along the propagation direction and they impact the specimen with a time delay of $1.6 \mathrm{~ns}$. The interaction among them is therefore negligible in vacuum, but a leading electron produces excitations in the sample that a second trailing projectile can pick up, whenever they last more than the delay time. This situation is discussed in Sec. V.B.1.

Another type of vicinage effect involves the electron self-interaction, taking place when its wave function extends over different regions of the sample. Current technology makes the observation of this effect feasible, and it should find direct application to measure nonlocal screened interactions, as shown in Sec. V.B.2.

\section{Interaction between two electrons}

This effect bears close relation to the amply discussed topic of swift charged-molecule dissociation after traversing thin foils, in which the interaction among the resulting ionic fragments affects their stopping (Gemmell et al., 1975) and the SE yield (Tomita et al., 2006). Likewise, mutual interaction between bunched electrons is at the heart of super-radiant Smith-Purcell emission (Urata et al., 1998; Li et al., 2006) as discussed in subsection of Sec. IV.E.

We can easily find from Eq. (8) the overall loss probability for two electrons moving a distance $a$ apart from each other along the same straight-line trajectory. The ratio of this probability to that of a single electron is exactly given by $4 \cos ^{2}(\omega a / 2 v)$ independent of the nature of the sampled excitations. When the electrons move in close proximity as compared to $v / \omega$, a twofold increase in the combined loss probability is produced. An interesting situation occurs under the condition $a$ $=\pi v / \omega$ : the energy released from the leading probe is picked up by the trailing one and the overall probability vanishes. This may find application to shadow intense energy-loss or light-emission features in order to make weaker spectral details more visible, in the spirit of the masking interferometry techniques utilized in astronomy (Ireland et al., 2008).

\section{Electron self-interaction}

The quantum nature of the electron probe can be exploited to retrieve nonlocal information on the dielectric response if we separate the incident beam into two or more coherent spots at the position of the sample. ${ }^{23}$ This is routinely done in TEM holography by inserting a prism in the electron optics, basically exploiting the partial coherence of the energy-loss signal (Schattschneider and Werner, 2005; Hartschuh, 2008; Verbeeck et al., 2008). Inelastic holography has been performed both in the real image plane (Lichte and Freitag, 2000) and in the diffraction plane (Herring, 2005), although the current theoretical understanding of the results obtained suggests that further development of the technique is required in order to extract new information on the coherence of inelastic excitations (Verbeeck, 2006). This technique permits addressing profound questions on quantum mechanics, such as the Feynman thought experiment (Potapov et al., 2007).

Here we discuss a similar scenario, consisting of an electron beam split into two coherent beams that are focused on identical neighboring spots. This situation is well described by Eq. (17) if one assumes that the lateral wave function of the incident electron has the form

$$
\psi_{i \perp}(\mathbf{R})=\frac{1}{\sqrt{2}}\left[F\left(\mathbf{R}-\mathbf{R}_{1}\right)+F\left(\mathbf{R}-\mathbf{R}_{2}\right) e^{i \delta}\right],
$$

where $F$ gives the spot profile (e.g., a Gaussian of width $\Delta R), \mathbf{R}_{j}$ are the centers of the two separate spots $\left(\mid \mathbf{R}_{1}\right.$ $-\mathbf{R}_{2} \mid \gg \Delta R$ ), and the phase $\delta \approx m_{e} v a / \hbar$ accounts for the difference in path length between the two beams $(a)$, which can be controlled through the electron optics of the microscope.

We now make two assumptions regarding the spot size: (1) the induced part of the screened interaction $W^{\text {ind }}$ is considered to be a smooth function that does not vary significantly over the extension $\Delta R$; (2) the lateral variation of the detected plane wave components across a distance $\Delta R$ is negligible, that is, $p_{f \perp} \Delta R \ll 1$ in Eq. (17). The first assumption limits our analysis to excitations extended over large regions as compared to the size of each beam spot. This is quite reasonable for a realistic value of $\Delta R \sim 2 \AA$ since most valence excitations including plasmons are extended over much larger distances (see, for example, Fig. 16). The second assumption defines the angular region of transmitted electrons in which we are interested (for instance, $p_{f \perp} \ll 1 / \Delta R$ $\sim 0.5 \AA^{-1}$ or, equivalently, collection angles $\ll 7 \mathrm{mrad}$ for $100 \mathrm{keV}$ electrons).

Inserting Eq. (64) into Eq. (17), one finds

$$
\begin{aligned}
\frac{d \Gamma^{\mathrm{NR}}(\omega)}{d \mathbf{p}_{f \perp}} \propto & \Gamma_{\mathrm{EELS}}^{\mathrm{NR}}\left(\mathbf{R}_{1}, \omega\right)+\Gamma_{\mathrm{EELS}}^{\mathrm{NR}}\left(\mathbf{R}_{2}, \omega\right) \\
& +\Gamma_{\mathrm{int}}^{\mathrm{NR}}\left(\mathbf{R}_{1}, \mathbf{R}_{2}, \omega\right),
\end{aligned}
$$

where

\footnotetext{
${ }^{23}$ Nonlocality here refers to $W\left(\mathbf{r}, \mathbf{r}^{\prime}, \omega\right)$ for $\mathbf{r} \neq \mathbf{r}^{\prime}$ and not to spatial dispersion in $\epsilon$.
} 


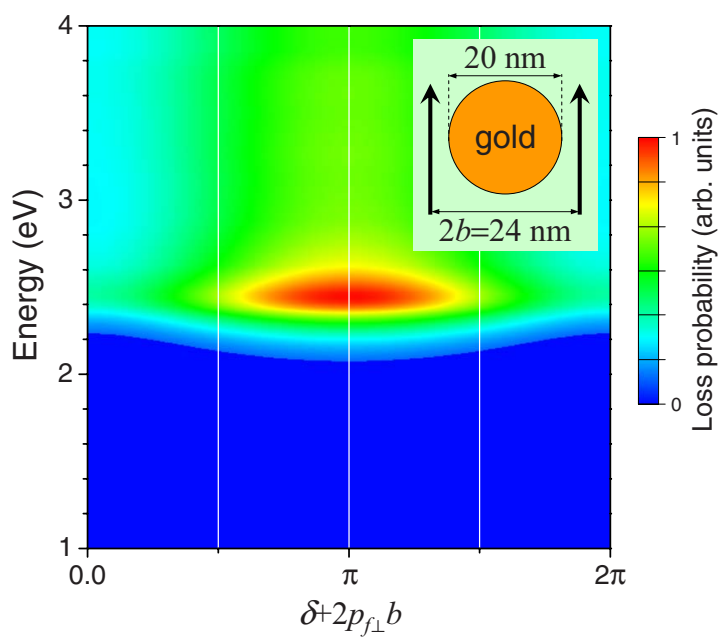

FIG. 31. (Color online) Electron self-interaction mediated by a metallic nanoparticle. Loss probability according to Eq. (65) for two coherent electron beams passing close to opposite sides of a $20 \mathrm{~nm}$ gold sphere (see inset). The gold is described with the dielectric function of Johnson and Christy (1972). The electron energy is $200 \mathrm{keV}$.

$$
\begin{aligned}
\Gamma_{\mathrm{int}}^{\mathrm{NR}}\left(\mathbf{R}_{1}, \mathbf{R}_{2}, \omega\right)= & \frac{2 e^{2}}{\pi \hbar v^{2}} \int d z_{1} d z_{2} \operatorname{Im}\left\{-W\left(\mathbf{r}_{1}, \mathbf{r}_{2}, \omega\right)\right\} \\
& \times \cos \left(\delta+\mathbf{p}_{f \perp} \cdot\left(\mathbf{R}_{1}-\mathbf{R}_{2}\right)\right. \\
& \left.-\frac{\omega}{v}\left(z_{1}-z_{2}\right)\right)
\end{aligned}
$$

contains the interference between the two spots.

Unlike the EELS probability $\Gamma_{\mathrm{EELS}}^{\mathrm{NR}}(\mathbf{R}, \omega)$ of Eq. (10), obtained when the entire inelastic signal is collected, the quantity $d \Gamma^{\mathrm{NR}}(\omega) / d \mathbf{p}_{f \perp}$ offers direct access to the nonlocal dependence of the screened interaction $\operatorname{Im}\left\{W\left(\mathbf{r}_{1}, \mathbf{r}_{2}, \omega\right)\right\}$. This requires exquisite wave-vector resolution $\Delta p_{f \perp} \ll 1 /\left|\mathbf{R}_{1}-\mathbf{R}_{2}\right|$ in order to prevent cancellations of the interference signal produced by the oscillations of the cosine function in $\Gamma_{\text {int }}^{\mathrm{NR}}$. Using a $100 \mathrm{keV}$ electron with an achievable angular resolution of $\varphi_{\text {out }}$ $\sim 10 \mu \mathrm{rad}$, one should be able to retrieve information for spots separated by a distance of $\sim 50 \mathrm{~nm}$, which is sufficient to study excitations of interest in nanoparticles and nanostructured materials.

We illustrate this formalism by considering two coherent beams of impact parameters $\mathbf{R}_{1}=b \hat{\mathbf{x}}$ and $\mathbf{R}_{2}=-b \hat{\mathbf{x}}$ relative to the center of a sphere of radius $a$, described by Eq. (B6). We obtain

$$
\begin{aligned}
\frac{d \Gamma_{\mathrm{sph}}^{\mathrm{NR}}(\omega)}{d \mathbf{p}_{f \perp}} \propto & \sum_{l=1}^{\infty} \sum_{m=-l}^{l} \frac{(\omega / v)^{2 l}}{(l+m) !(l-m) !} K_{m}^{2}\left(\frac{\omega b}{v}\right) \operatorname{Im}\left\{\alpha_{l}^{\mathrm{NR}}\right\} \\
& \times\left[1+(-1)^{m} \cos \left(\delta+2 p_{f \perp} b\right)\right]
\end{aligned}
$$

where $\alpha_{l}^{\mathrm{NR}}$ is defined in Eq. (32). Figure 31 represents the results obtained from Eq. (65) for a $200 \mathrm{keV}$ electron split into two beams that are passing at a distance of $2 \mathrm{~nm}$ from a $20 \mathrm{~nm}$ gold particle, as shown in the inset.

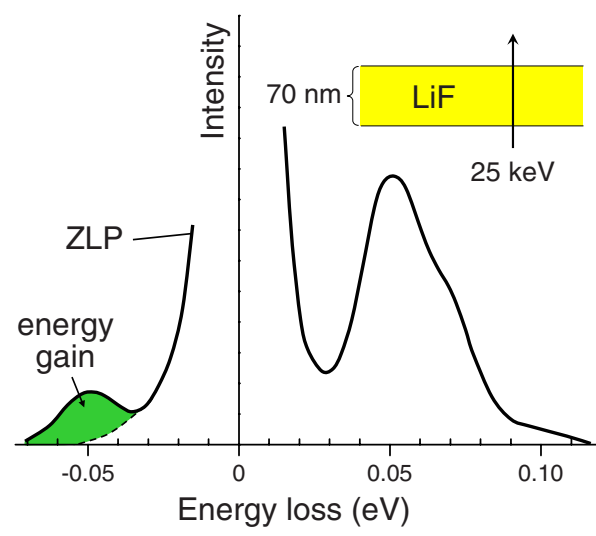

FIG. 32. (Color online) Pioneering evidence of energy gain after transmission of fast electrons through a thin foil. Energygain events involve room-temperature phonon absorption by the electron and are strongly reduced at lower temperature. Adapted from Boersch, Geiger, and Stickel, 1966.

Interestingly, $d \Gamma_{\mathrm{sph}}^{\mathrm{NR}} / d \mathbf{p}_{f \perp}$ is periodic in $p_{f \perp}$ with period $\pi / b$ and is dominated by a dipolar feature. Furthermore, the interference disappears under the condition $\delta$ $+2 p_{f \perp} b=(2 n+1) \pi / 2$ for integers $n$, leading to a loss probability proportional to Eq. (31). Full interference is obtained for $\delta+2 p_{f \perp} b=n \pi$, which results in partial suppression of the dipole mode.

\section{Electron energy-gain spectroscopy}

The width of the ZLP limits the resolution of EELS and masks low-energy features sitting in the elastic peak tail. These problems are partially solved as shown below if one is capable of reversing the process, so that energy gain rather than loss is recorded. Electron acceleration events were actually reported over 40 years ago when Boersch, Geiger, and Stickel (1966) observed absorption of thermal phonons by electrons transmitted through thin LiF films, as shown in Fig. 32. The gain signal was later shown to depend on temperature as

$$
\Gamma_{\mathrm{EEGS}}^{\text {thermal }}(\omega)=n(\omega) \Gamma_{\mathrm{EELS}}(\omega),
$$

where $\Gamma_{\text {EELS }}(\omega)$ is the EELS probability discussed in Sec. II and $n(\omega)=1 /\left[\exp \left(\hbar \omega / k_{B} T\right)-1\right]$ is the BoseEinstein distribution function at temperature $T$ (García de Abajo and Kociak, 2008a). Actually, Boersch, Geiger, and Stickel (1966) managed to suppress the measured $\Gamma_{\text {EEGS }}^{\text {thermal }}$ by cooling their samples. Unfortunately, thermal transfers are limited to modes of energies $\lesssim k_{B} T$ $\approx 26 \mathrm{meV}$ at room temperature.

Another strategy was successfully demonstrated by Schilling and Raether (1973), who managed to observe electron energy gain produced by SPs in a fraction of electrons reflected from an indium surface. Their SPs were created by other electrons of the same intense electron beam.

A more flexible strategy consists in exciting through external illumination only those modes that we want to probe, as suggested by Howie (1999, 2009). The pro- 


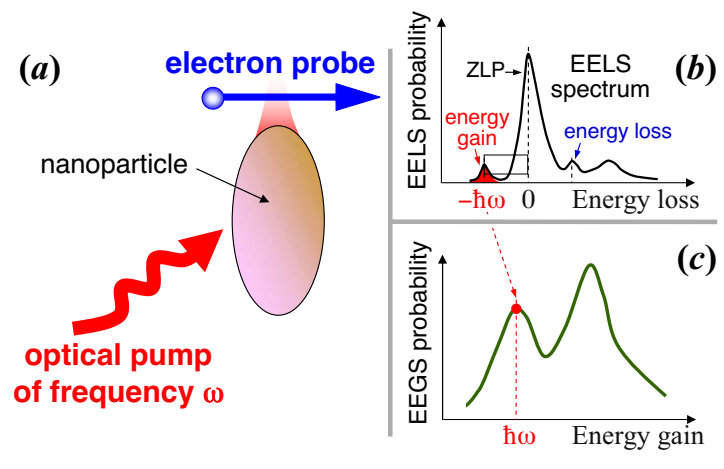

FIG. 33. (Color online) Schematic description of electron energy-gain spectroscopy (EEGS). (a) An electron passes near a nanostructure (a nanoparticle in the illustration) that is illuminated by light of frequency $\omega$. (b) A regular EELS spectrum acquired in a scanning transmission electron microscope (STEM) shows a gain peak on the left-hand side with respect to the zero-loss peak, corresponding to electrons that have absorbed one photon out of the external light. (c) The electron energy-gain probability for light of frequency $\omega$ is proportional to the area of the energy-gain peak. The gain process is assisted by localized excitations of the nanostructure. They supply the necessary momentum to couple evanescent scattered light to the electron, so that the EEGS probability exhibits features as a function of photon frequency mimicking the excitation modes of the sample.

posed technique is shown in Fig. 33 and can be termed electron energy-gain spectroscopy (EEGS) (García de Abajo and Kociak, 2008a), in which the electrons absorb photons from an external light source. However, the energy-momentum mismatch between freely propagating charges and photons prevents the linear coupling of fast electrons and light plane waves in vacuum (for example, an electron moving in vacuum cannot emit CR). ${ }^{24}$ Therefore, we must rely on the interaction with a material that is structured on subwavelength scales (the specimen under scrutiny) to produce evanescent optical fields carrying the necessary complex momentum to make this coupling feasible. This is already the case in the inverse Cherenkov effect, whereby an electron moving under the condition given by Eq. (7) can absorb energy from a laser aligned close to the Cherenkov cone, as proposed by More (1966) and observed 15 years later by Edighoffer et al. (1981). The inverse Cherenkov effect can find application to accelerate charged particles (Kimura et al., 1995). In a related context, the observation of the inverse Smith-Purcell effect (Mizuno et al., 1975, 1987) demonstrated the coupling of free electrons to evanescent components of momentum produced by interaction of external light with a grating.

Here we consider a more suitable scenario for spectroscopy. Light scattered from a nanostructure, and in particular evanescent components in its vicinity, possess the necessary momentum to couple to the electron. Con-

\footnotetext{
${ }^{24}$ In-vacuum optical acceleration of an electron moving along the axis of a Bessel beam has recently been proposed (Zhao and Lü, 2008).
}

sequently, the energy-gain process can occur near the specimen and should yield information on its spectral response, directly mapping the evanescent light strength. In such a setup, the full width of the gain peak must correspond to electrons that have absorbed one photon out of the external light, and therefore the energy resolution of this technique is only limited by the width of the external illumination, typically in the sub-meV domain (Rapoport and Khattak, 1988). This is a 100-fold increase in spectral resolution with respect to currently available EELS technology. [Notice that low-energy excitations (e.g., sub-meV modes) are difficult to resolve due to the ZLP background. Here we refer instead to the ability to resolve higher-energy excitation features (e.g., $1 \mathrm{eV}$ ) separated by minute energy differences (e.g., 1 vs $1.001 \mathrm{eV})$.]

In order to evaluate EEGS probabilities, we consider an energetic electron focused near the sample and describe it through the wave function of Eq. (16) (Sec. II.C.2). We adopt the nonrecoil approximation and assume that the normalized transverse wave function $\psi_{i \perp}(\mathbf{R})$ is focused within a narrow spot centered around $\mathbf{R}=\mathbf{R}_{0}$, so that only the motion along the beam direction $z$ needs to be considered. The electron wave function is perturbed by interaction with the external light field and picks up components of higher energy to become to first order in the perturbation

$$
\psi_{\|}(z)=\frac{1}{L^{1 / 2}} e^{i p_{i} z} e^{-i \varepsilon_{i} t}+\delta \psi_{\|}(z) e^{-i \varepsilon_{f} t}
$$

where we now have $\varepsilon_{f}=\varepsilon_{i}+\omega$ and $\omega$ is the photon frequency. Starting from the Lippmann-Schwinger equation and working to first-order perturbation theory, we find

$$
\delta \psi_{\|}(z)=\int d z^{\prime} G_{f}\left(z-z^{\prime}\right) H_{\omega}\left(z^{\prime}\right) \frac{e^{i p_{i} z^{\prime}}}{L^{1 / 2}}
$$

where $H_{\omega}(z)=\left(-e \hbar / m_{e} \omega\right) E_{z}\left(\mathbf{R}_{0}, z\right) \partial_{z}$ is the energy-gain $[\propto \exp (-i \omega t)]$ part of the electron-photon coupling Hamiltonian, $E_{z}$ is the $z$ component of the light electric field (parallel to the trajectory), $\partial_{z}$ denotes differentiation with respect to $z$, and

$$
\begin{aligned}
G_{f}\left(z-z^{\prime}\right) & =\int \frac{d p}{2 \pi} \frac{e^{i p\left(z-z^{\prime}\right)}}{\left(\hbar \varepsilon_{f}-\hbar^{2} p^{2} / 2 m_{e}+i 0^{+}\right)} \\
& =-\frac{i m_{e}}{p_{f} \hbar^{2}} e^{i p_{f}\left|z-z^{\prime}\right|}
\end{aligned}
$$

is the nonrelativistic Green's function of the free electron propagating along the $z$ direction with final energy $\hbar \varepsilon_{f}$. The integral in Eq. (66) must be performed in the $z \rightarrow \infty$ limit in order to ensure that the electron has already abandoned the region of interaction with the nanostructure. Then $\delta \psi_{\|}$can be expressed as a coefficient multiplying a plane wave function of energy $\hbar \varepsilon_{f}$. Finally, the gain probability is given by the square of that coefficient as the dimensionless quantity, 


$$
P_{\mathrm{EEGS}}(\omega)=\left(\frac{e}{\hbar \omega}\right)^{2}\left|\int d z e^{-i \omega z / v} E_{z}\left(\mathbf{R}_{0}, z\right)\right|^{2}
$$

where we have used the nonrecoil approximation $p_{f}$ $-p_{i} \approx \omega / v \ll p_{i}$ for the electron moving with high velocity $v=\hbar p_{i} / m_{e}$. If the incident field is a plane wave and the electron moves in vacuum, only scattered components contribute to the EEGS probability because the momentum gained by the electron $(\hbar \omega / v)$ is always larger than the free-space momentum of light. Therefore, we can substitute the induced field for the total field in Eq. (67).

Stimulated photon emission (energy loss) can also take place by interaction with the $\exp (i \omega t)$ part of the electron-photon coupling Hamiltonian. One obtains an expression identical to Eq. (67), but with $\exp (-i \omega z / v)$ changed to $\exp (i \omega z / v)$.

Useful analytical formulas can be derived for small particles, in which the dipolar response is dominant, via their electric polarizability $\alpha$. The EEGS probability is given by integration of the dipole scattered field,

$$
E_{z}^{\text {ind }}=\alpha(\omega)\left[k^{2} E_{z}^{\mathrm{ext}}+\left(\mathbf{E}^{\mathrm{ext}} \cdot \boldsymbol{\nabla}\right) \partial_{z}\right] \exp (i k r) / r .
$$

Direct application of Eq. (67) yields (for linearly polarized light)

$$
\begin{aligned}
P_{\text {EEGS,dip }}(\omega)= & \left|\frac{2 e \omega \alpha}{\hbar v^{2} \gamma}\right|^{2}\left[\left|\mathbf{E}^{\text {ext }} \cdot \hat{\mathbf{b}}\right|^{2} K_{1}^{2}\left(\frac{\omega b}{v \gamma}\right)\right. \\
& \left.+\frac{\left|E_{z}^{\mathrm{ext}}\right|^{2}}{\gamma^{2}} K_{0}^{2}\left(\frac{\omega b}{v \gamma}\right)\right],
\end{aligned}
$$

where $\mathbf{b}$ is the impact parameter of the electron relative to the particle $(\mathbf{b} \perp \hat{\mathbf{z}})$ and $\hat{\mathbf{b}}=\mathbf{b} / b$. Interestingly, for an external field that is perpendicularly oriented with respect to the electron trajectory, the scattered field picks up $z$ components that couple to the electron, giving rise to the $K_{1}^{2}$ contribution in Eq. (68). This expression needs to be compared with the EELS probability for a small particle $\left[\Gamma_{\text {EELS,dip }}\right.$; Eq. (34)]. For a lossless particle, the optical theorem $\left[\operatorname{Im}\{\alpha\}=\left(2 k^{3} / 3\right)|\alpha|^{2}\right]$ allows us to write $\Gamma_{\text {EELS,dip }}=\left\langle P_{\text {EEGS,dip }}\right\rangle \rho^{0} f$, where $\rho^{0}=\omega^{2} / \pi^{2} c^{3}$ is the freespace local density of photonic states, the factor $f=2 \pi \hbar \omega /\left|E^{\mathrm{ext}}\right|^{2}$ corrects for the number of photons contained in the external field $\mathbf{E}^{\text {ext }}$, and the average over orientations of this field has been performed. In other words, the EELS probability or equivalently the CL probability $\left(\Gamma_{\mathrm{CL}}\right)$ in our lossless particle coincides with the EEGS probability for an incoherent source containing one photon per photon state. For a dissipative particle, we have $\Gamma_{\mathrm{EELS}}>\Gamma_{\mathrm{CL}}$, but the relation $\Gamma_{\mathrm{CL}}$ $=\left\langle P_{\text {EEGS,dip }}\right\rangle \rho^{0} f$ still holds.

We are now ready to make a quantitative comparison of EEGS and EELS. Incidentally, $\Gamma_{\text {EELS }}$ has units of inverse frequency, whereas $P_{\text {EEGS }}$ is dimensionless, so that the comparison requires integrating $\Gamma_{\text {EELS }}$ over a finite frequency-loss range $\Delta \omega$. We have $P_{\text {EEGS }} /\left(\Delta \omega \Gamma_{\text {EELS }}\right)$ $\sim\left|E^{\operatorname{ext}}\right|^{2} /[\hbar \Delta \omega \operatorname{Im}\{-1 / \alpha\}]$. For instance, for a $100 \mathrm{~nm}$ gold nanoparticle illuminated with a laser of moderate intensity $\sim 10^{6} \mathrm{~W} / \mathrm{cm}^{2}$, tuned to the particle plasmon en- ergy $\hbar \omega=2.5 \mathrm{eV}$, and taking $\hbar \Delta \omega=0.1 \mathrm{eV}$ (the plasmon width), we find $P_{\mathrm{EEGS}} /\left(\Delta \omega \Gamma_{\mathrm{EELS}}\right) \approx 40$. This must be compared with $\Gamma_{\mathrm{CL}} / \Gamma_{\mathrm{EELS}}<1$.

We conclude that EEGS should provide an excellent probe of local photonic properties, combining the spatial resolution of TEMs and the energy resolution of optical sources. The EEGS signal is free from bulk features that dominate the loss spectrum as soon as the electron trajectory intersects the sample. Temporal control over the intensity of the pumped light adds an extra handle to facilitate the detection of the gain signal in contrast to CL. Finally, EEGS is sensitive to light polarization and it can be applied to investigate nonlinear optical response with nanometer resolution.

Recently, a new report has shown imaging of nanostructures using femtosecond electron pulses in coincidence with femtosecond light pulses, which produce sufficiently intense electron-photon coupling to enable images to be constructed from the energy-gain part of the transmitted electron spectrum (Barwick et al., 2009).

\section{Surface plasmon launching}

As microchip features continue to shrink, lithographically patterned metal structures are becoming natural candidates to replace electronic microcircuits. The new devices are expected to operate at frequencies above the terahertz rather than gigahertz and to encode information in plasmon signals rather than electric currents. The field of plasmonics is actually experiencing an expansion in preparation for this emerging technology (Barnes et al., 2003) but is still facing the lack of suitable plasmon sources that provide the versatility demanded by highly integrated plasmonic circuits, which conventional techniques such as frustrated total internal reflection and NSOM cannot satisfy.

Fast electrons are a promising alternative for generating plasmons with exquisite spatial control and relatively high efficiency (Adamo et al., 2009). Plasmon launching by swift electrons was demonstrated several decades ago by Teng and Stern (1967) and Heitmann (1977) in metallic gratings. They utilized the periodic corrugation to couple plasmons to propagating light, which was analyzed in energy and angle of emission.

These pioneering studies have recently been supplemented by monitoring SPP generation in metallic planar surfaces. The SPPs were eventually decoupled by means of a grating placed at some distance from the position in which they were generated (Bashevoy et al., 2006; van Wijngaarden et al., 2006), thus yielding information on SPP propagation lengths through the dependence of the resulting emission intensity on the distance between the beam and the grating and conclusively demonstrating the excitation of SPPs by fast electrons. Subsequent studies have added more insight into the spectrally and spatially resolved propagation of SPPs (Bashevoy et al., 2007; Yamamoto and Suzuki, 2008; Kuttge, Vesseur, Koenderink et al., 2009; Vesseur, García de Abajo, and Polman, 2009), the increase in plasmon losses produced by grain boundaries in polycrystalline gold compared to 

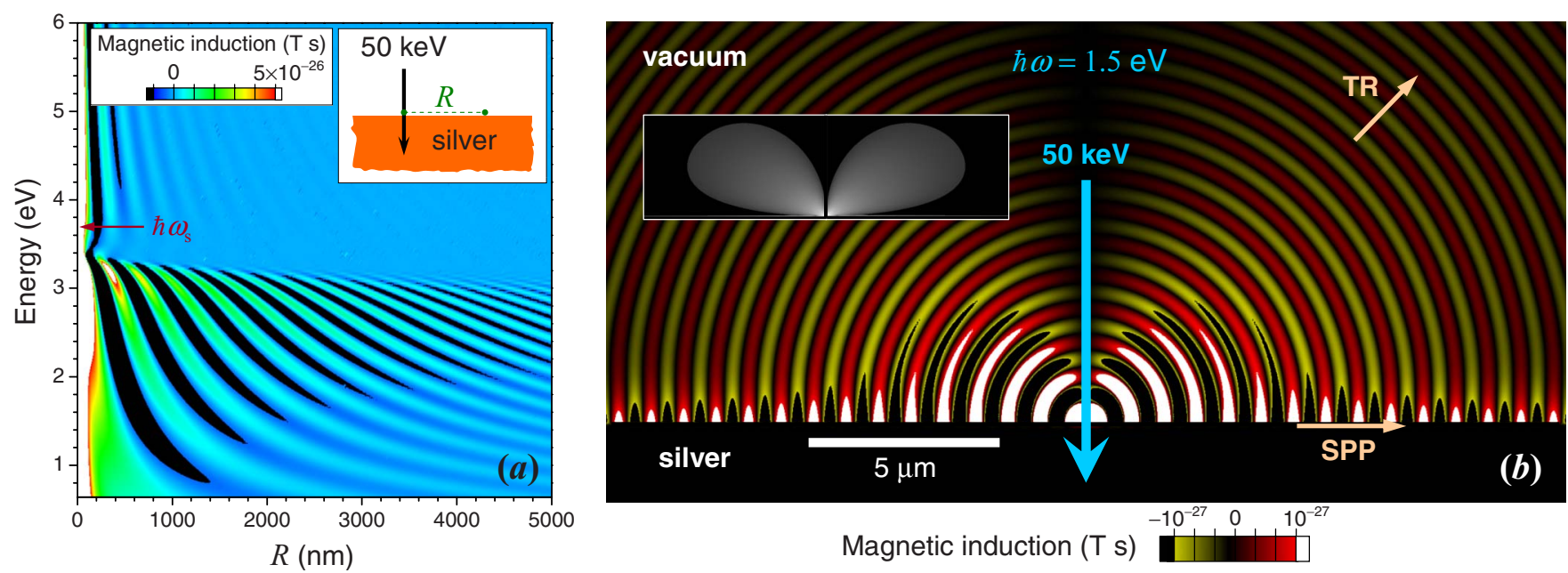

FIG. 34. (Color online) Spatial visualization of plasmon launching. (a) The magnetic induction $\mathbf{B}(\mathbf{r}, \omega)$ set up by an electron impinging on a semi-infinite silver sample [see Eq. (55)] is represented as a function of distance to the trajectory along the surface and is resolved in energy components $\hbar \omega$. Plasmon generation leads to oscillations of increasing wavelength with decreasing plasmon energy, in agreement with the dispersion relation of Fig. 11. The induction is calculated at a distance of $10 \mathrm{~nm}$ from the surface. (b) Full spatial dependence of the $\hbar \omega=1.5 \mathrm{eV}$ component of the magnetic induction under the same conditions as in (a). The left inset shows a logarithmic scale overview stretching $1.2 \mathrm{~mm}$ along the surface, in which SPP and TR components are clearly separated. The dielectric function of Ag is taken from Johnson and Christy, 1972.

single-crystal gold (Kuttge et al., 2008), the excitation near the plasmon threshold $\omega_{s}$ (Peale et al., 2008), or the launching of metal-insulator-metal plasmons (Cai et al., 2009; Kuttge, Cai, et al., 2009).

Plasmons can be identified in Eq. (51) with the pole in the denominator of $D$ [Eq. (54)], which yields the dispersion relation given by Eq. (27). The $q_{\|}$integral of Eq. (55) can be then performed in the $R \gg \lambda$ limit by only retaining the contribution of the $q_{\|}=q_{\|}^{\mathrm{SP}}$ pole. This is the so-called plasmon-pole approximation (Ford and Weber, 1984), which enables us to approximate

$$
D \approx \frac{C}{q_{\|}-q_{\|}^{\mathrm{SP}}},
$$

with

$$
C=\frac{2 i e q_{z 1}^{\mathrm{SP}}}{c} \frac{\epsilon_{2}}{\epsilon_{1}^{2}-\epsilon_{2}^{2}} .
$$

(This comes from the Taylor expansion of $\epsilon_{2} q_{z 1}+\epsilon_{1} q_{z 2}$ around the $q_{\|}=q_{\|}^{\mathrm{SP}}$ pole.) Now, we proceed by inserting the plasmon-pole expression back into the reflected components of the fields [Eq. (55)] and by approximating the integral over $q_{\|}$using the $q_{\|}=q_{\|}^{\text {SP }}$ pole of Eq. (69). Finally, Eq. (55) reduces to

$$
\mathbf{H}^{\mathrm{ref}}(\mathbf{r}, \omega) \approx A_{j} e^{i q_{z j}^{\mathrm{SP}}|z|} H_{1}^{(1)}\left(q_{\|}^{\mathrm{SP}} R\right) \hat{\varphi},
$$

where $q_{z j}^{\mathrm{SP}}=\sqrt{k^{2} \epsilon_{j}-\left(q_{\|}^{\mathrm{SP}}\right)^{2}}$,

$$
A_{j}=\pi q_{\|}^{\mathrm{SP}} \operatorname{sgn}(z) C \mu_{j},
$$

and $\mu_{j}$ is defined in Eqs. (52) and (53). Here $H_{1}^{(1)}$ is a Hankel function, dying off as $\approx-\left(2 i / \pi q_{\|}^{\mathrm{SP}} R\right)^{1 / 2}$ $\times \exp \left(i q_{\|}^{\mathrm{SP}} R\right)$ at large distance $R$ from the electron trajectory $^{25}$ (Abramowitz and Stegun, 1972). Equation (70) indicates that the induced plasmon field is a cylindrical wave of $m=0$ symmetry and parallel wave vector $q_{\|}^{\mathrm{SP}}$.

Figure 34(a) shows the real part of the reflected magnetic induction $\left[\mathbf{B}^{\mathrm{ref}}(\mathbf{r}, \omega)\right]$, calculated from Eqs. (54) and (55) as a function of $R$ and $\omega$ for $\mathbf{r}=(R, 0,10 \mathrm{~nm})$ along a silver surface bombarded by a $50 \mathrm{keV}$ electron. Surface plasmons produce strong modulations of the field at energies below Ritchie's nonretarded limit $\left(\hbar \omega_{s} \approx 3.7 \mathrm{eV}\right.$ for $\mathrm{Ag}$ ). The period of these oscillations increases with decreasing $\omega$ in accordance with the $H_{1}^{(1)}$ behavior noted above. The surface field exhibits faster oscillations for $\omega>\omega_{s}$, which rapidly decay away from the trajectory. The full spatial dependence of $\mathbf{B}^{\mathrm{ref}}(\mathbf{r}, \omega)$ is shown in Fig. 34 (b) for $\hbar \omega=1.5 \mathrm{eV}$, showing the excitation of a cylindrical SPP wave (see intensity maxima near the surface) and TR (oscillatory wave components evolving away from the surface). The TR region is dominated by oscillations of long wavelength $(2 \pi / k)$ compared to the SPP wavelength $\left[2 \pi / \operatorname{Re}\left\{q_{\|}^{\mathrm{SP}}\right\}\right]$, in consonance with the larger momentum of SPPs $\left[\operatorname{Re}\left\{q_{\|}^{\mathrm{SP}}\right\}>k\right]$.

We are ultimately interested in determining how many plasmons are launched per incident electron, which can be calculated from the integral of the Poynting vector derived from Eq. (70) over a cylindrical sur-

\footnotetext{
${ }^{25}$ The $1 / \sqrt{R}$ dependence of the field is the signature of a surface mode since the resulting Poynting vector scales as $1 / R$, so that its integral over a circle of radius $R$ centered at the electron source stays independent of $R$ except for the exponential attenuation due to nonvanishing SPP absorption, $\exp \left[-2 \operatorname{Im}\left\{q_{\|}^{\mathrm{SP}}\right\} R\right]$.
} 
face centered around the electron trajectory. This leads to

$$
\Gamma_{\mathrm{SP}}(\omega)=\frac{1}{2 \pi^{2} \hbar k^{2}} \sum_{j=1,2} \frac{\left|A_{j}\right|^{2}}{\operatorname{Im}\left\{q_{z j}^{\mathrm{SP}}\right\}} \operatorname{Re}\left\{\frac{q_{\|}^{\mathrm{SP}}}{\left|q_{\|}^{\mathrm{SP}}\right| \epsilon_{j}}\right\}
$$

for the probability normalized per incident electron and per unit of plasmon frequency range. ${ }^{26}$

The prediction of Eq. (71) is represented in Fig. 23(a) (solid curves) for $\mathrm{Au}$ and $\mathrm{Ag}$ surfaces bombarded by $200 \mathrm{keV}$ electrons and normalized per unit of emission wavelength $\lambda$ [i.e., the plotted quantity is $\left.(\omega / \lambda) \Gamma_{\mathrm{SP}}(\omega)\right]$. Plasmon production is boosted near $\omega_{s}$ in both cases, and it actually surpasses the production of TR in that region. Roughly speaking, $\sim 10^{-3}$ gold plasmons are produced for each normally incident electron within the $600-1200 \mathrm{~nm}$ spectral range, a value that reduces to $\sim 1.7 \times 10^{-4}$ for $20 \mathrm{keV}$ incident energy and monotonically grows with electron velocity until it levels up at a value of $\sim 2 \times 10^{-3}$ around $2 \mathrm{MeV}$ [Fig. 23(b)]. This behavior is qualitatively consistent with the spatial extension of the external electron field $\sim v \gamma / \omega$ [see Eq. (5)], and one should expect maximum coupling to the plasmon field when that extension is of the order of the SPP wavelength. The dependence of the emission on primary electron energy, plotted in Fig. 23(b), confirms this explanation.

Good agreement between the above theory and experiment has recently been obtained by Kuttge, Vesseur, Koenderink, et al. (2009), who inferred the absolute SPP yield by first normalizing the measured CL signal to the calculated TR in a flat surface and then analyzing the interference between SPPs decoupled by a grating and TR. The results are shown in Fig. 23(c).

Propagating plasmons can be generally excited with electron beams in any plasmon-supporting system, including metal films, nanowires, and particle arrays (Cai et al., 2009). An example of plasmon excitation in a linear particle array is shown in Fig. 17, whereas experimental observation of electron coupling to plasmons in nanowires was obtained by Yamamoto, Nakano, and Suzuki (2006) and Vesseur et al. (2007).

The emission mechanism so far considered is coherent in the sense explained in Sec. IV.A.1. However, there are at least two other incoherent mechanisms of emission involving plasmons: (1) plasmon-assisted decay of localized excitations produced by the impinging electron close to its trajectory and (2) SEs, which can in turn couple to the plasmon, acting as primary electrons. These contributions are only effective within a distance of the order of the skin depth inside the metal. We can invoke Bethe's theory of charge stopping (Bethe, 1930) to conclude that the energy deposited within that region scales as $\sim 1 / E$ with the primary electron energy $E$. Consequently, one should observe relatively more inco-

\footnotetext{
${ }^{26}$ It should be noted that the approximation of Eq. (69) is fully justified for $R \gg \lambda$, that is, in the limit that we have examined to derive the plasmon energy flux far away from the electron trajectory (García de Abajo, 2007).
}

herent emission at low electron energies and an increase in the degree of coherence with faster electrons.

\section{E. Nonlocal effects in nanostructured metals}

The interaction between closely spaced particles has recently attracted much interest because they produce large electric field enhancement in the gap region, which is beneficial for molecular sensing applications ( $\mathrm{Xu}$ et al., 1999). Gap distances in the range of one to a few nanometers have actually been used to measure surfaceenhanced Raman scattering (SERS) signals (RodríguezLorenzo et al., 2009), but nonlocal effects have been largely neglected in this context until recently (García de Abajo, 2008; Zuloaga et al., 2009). Spatially resolved EELS is well suited to experimentally study these unexplored nonlocal effects in dimers, and therefore we discuss this problem in this section.

Spatial dispersion in the material response generally produces blueshifts and decreasing intensity of plasmon features. Since the early work of Fuchs and Kliewer (1968) on spheres, nonlocal effects in EELS have been theoretically addressed for several geometries in the nonretarded limit, and they have been predicted to show up for electron impact parameters in the nanometer range (Zabala and Echenique, 1990; Aizpurua and Rivacoba, 2008).

Nonlocal effects in the optical response of small metal spheres $(\leqslant 10 \mathrm{~nm})$ have been known for a long time [see, for example, Ouyang et al. (1992) for an EELS experimental study of small silver particles], and they are well described by a phenomenological model incorporating conduction-electron scattering at the particle boundary as a source of plasmon decoherence (Kreibig and Vollmer, 1995). This model consists in supplementing the damping rate $\eta$ in Eq. (A1) with a sizedependent term $v_{F} / a$. However, the $v_{F} / a$ quantumconfinement model is inconsistent with the fact that an electron state trapped inside a finite cavity (mimicking a conduction electron in a nanoparticle) has infinite lifetime (apart from small radiative decay broadening of excited states). Therefore, many-body effects rather than reflection at the boundaries seem to be the source of dephasing in small particles, facilitated by spatial dispersion, which is responsible for the breakdown of the local response approximation at distances below a few nanometers (García de Abajo, 2008).

The hydrodynamic model offers an alternative way of accounting for nonlocal effects, as discussed in Appendix A.2 for a general case and in Appendix $\mathrm{C}$ for spheres. The results of this model for individual aluminum spheres are shown in Fig. 14(a) (dashed curves), compared with a local description (solid curves). As expected, nonlocal calculations are blueshifted with respect to the local theory.

For small gold dimers at optical frequencies, it is reasonable to describe the particles using Eq. (37) and to plug the resulting multipolar polarizability into the multiple-scattering formalism of Sec. III.E.4. We have used Eq. (21) for $\epsilon(q, \omega)$. The calculated loss probability 


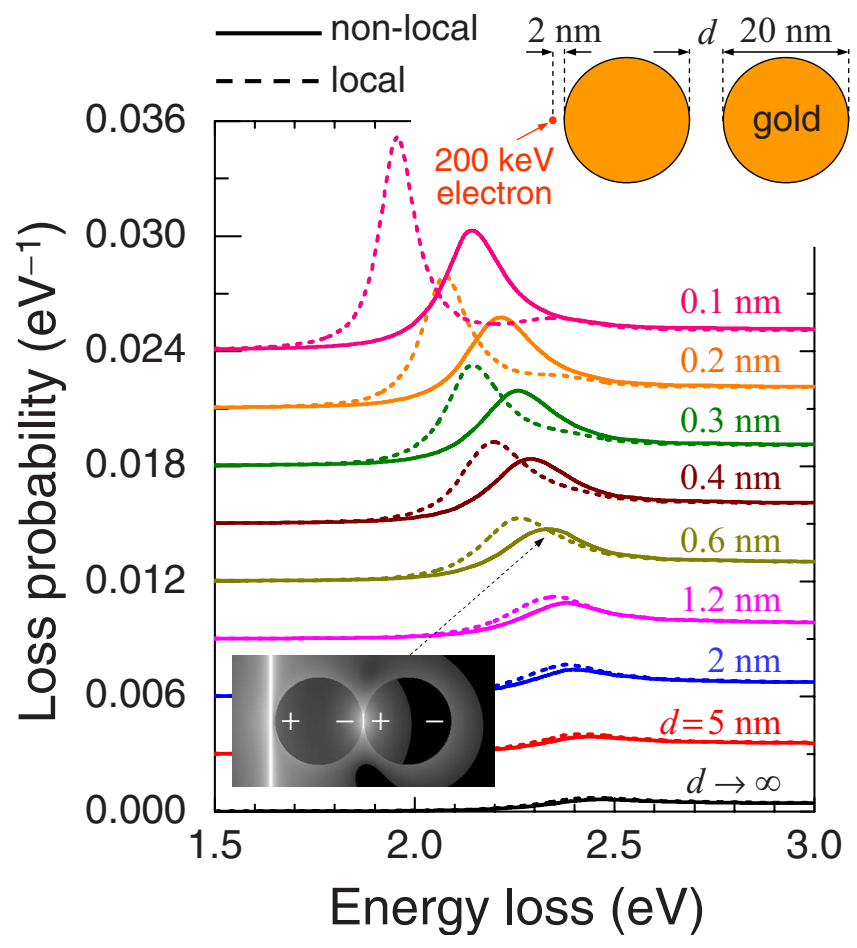

FIG. 35. (Color online) Probing nonlocal effects through EELS in metal nanoparticle dimers. Loss probability of a $200 \mathrm{keV}$ electron passing near dimers formed by spherical gold particles of $20 \mathrm{~nm}$ in diameter. The loss spectra are dominated by the longitudinal dipole mode. Local (dashed curves) and nonlocal (solid curves) calculations are compared for several separations between the particle surfaces $d$. Consecutive curves are vertically offset by $0.003 \mathrm{eV}^{-1}$ for clarity. The electron trajectory is perpendicular to the dimer axis and located as indicated in the upper right inset. The lower left inset shows the $\hbar \omega=2.26 \mathrm{eV}$ component of the total-field intensity in logarithmic scale for $d=0.6 \mathrm{~nm}$ calculated from the BEM; the field diverges along the electron trajectory (vertical bright line) and it has a local maximum in the dimer-gap region.

is represented in Fig. 35 for $20 \mathrm{~nm}$ particles (see García de Abajo, 2008, for more details on the model). The loss spectra show a dominant feature that corresponds to the lowest-order longitudinal dipole mode of the structure [i.e., this mode corresponds to the coaxial dipole-dipole mode characterized by a charge pattern $(+-) \cdot(+-)$ at large separations]. Significant enhancement of the electric field is produced near the gap region at the plasmon mode frequency (Fig. 35, lower left inset), so that the details of the dielectric response at small distances become critical. The local description of the sphere (dashed curves) is insufficient at distances below $2 \mathrm{~nm}$ since they produce redshift and increase of intensity ascompared to the nonlocal results (solid curves). The response of the dimer is extremely sensitive to the gap distance, but electron microscopes are ideally suited to simultaneously determine this parameter and the plasmon energy.

\section{PROSPECTS FOR PLASMONICS}

Browsing the figures of this review, we observe that the historical development of plasmons has been inti- mately correlated with advances in electron microscopy and spectroscopy. Since the early days of SPs, which Ritchie (1957) predicted to show up as characteristic energy losses in electrons traversing metallic thin foils (see Fig. 10), until now that particle plasmons can be mapped with nanometer precision (see Fig. 16), these collective excitations have captured the imagination of electron microscopists and material scientists alike.

Plasmons have now taken off as good tools in ultrafast technology for processing information encoded as plasmonic signals (Barnes et al., 2003; Ozbay, 2006; Zia, 2006) and for applications in biology (Schuster et al., 1993) and biomedicine (Burda et al., 2005; Gobin et al., 2007). In particular, localized plasmon resonances (LPRs) play a relevant role because they are typically accompanied by large enhancement of the electromagnetic field that is useful in molecular sensing [for example, using SERS (Xu et al., 1999)]. ${ }^{27}$ It is of course important to have experimental tools that permit us to visualize LPRs in patterned metallic surfaces and nanoparticles. One of these tools is NSOM (see Kim and Song, 2007, and references therein), which has been successfully applied to map plasmons (Bozhevolnyi et al., 2006; Drezet et al., 2007) although it is limited to around several tens of nanometers in spatial resolution. In this context, absorption and scattering spectroscopy has been extensively used to characterize the optical properties of metallic nanoparticles in colloids (Burda et al., 2005), but it requires narrow distributions of particle size and morphology, and it can only access the average properties of particle assemblies. Similarly, dark-field microscopy (DFM) is becoming a popular technique in the colloidal chemistry community, allowing localized plasmons to be studied in individual nanoparticles (Mock et al., 2002). However, unlike EELS, the DFM

\footnotetext{
${ }^{27}$ Localized plasmons can be confined down to a few nanometers in small particles, for which retardation effects are negligible, so that LPRs are safely described by Poisson's equation. Actually, the minimum size of well-defined LPRs is limited only by broadening due to nonlocal effects (see Sec. V.E). Now, the question arises, are metals (in the optical sense) necessary to produce localized electromagnetic resonances? In other words, do we need to have negative $\epsilon$ materials? A positive answer to this question comes from examining the eigenvalues $\Lambda=2 \pi\left(\epsilon_{2}+\epsilon_{1}\right) /\left(\epsilon_{2}-\epsilon_{1}\right)$ that appear in the solution of Poisson's equation, recast into Eq. (38) (i.e., the solution corresponding to vanishing external field): one finds that the eigenvalues are real and they must satisfy $|\Lambda|<2 \pi$, which is possible only if $\epsilon_{1}$ and $\epsilon_{2}$ have opposite signs. An alternative explanation based on physical intuition can be found in the formal equivalence between Poisson's equation and the heat transport equation under stationary conditions, with $\epsilon$ and $\phi$ playing the role of the thermal conductivity and the temperature, respectively: real materials have positive thermal conductivity, which guarantees that the second law of thermodynamics is fulfilled (heat does flow toward regions of lower temperature); but we know that heat cannot be spatially confined forever using passive materials, so that $\epsilon>0$ media are unable to trap the nonuniform $\phi$ associated with a localized resonance.
} 


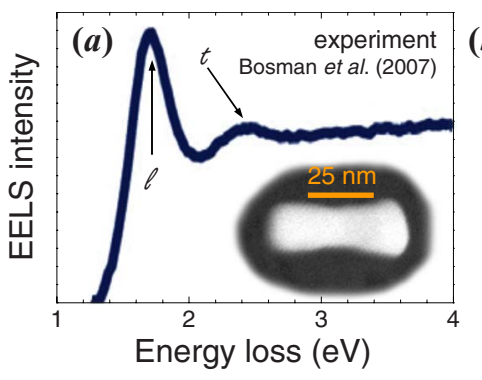

(d)
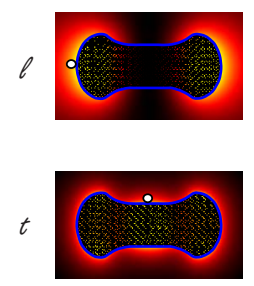

(e)
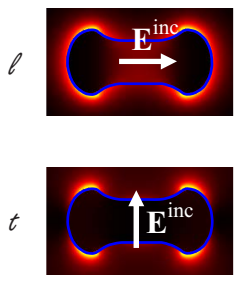
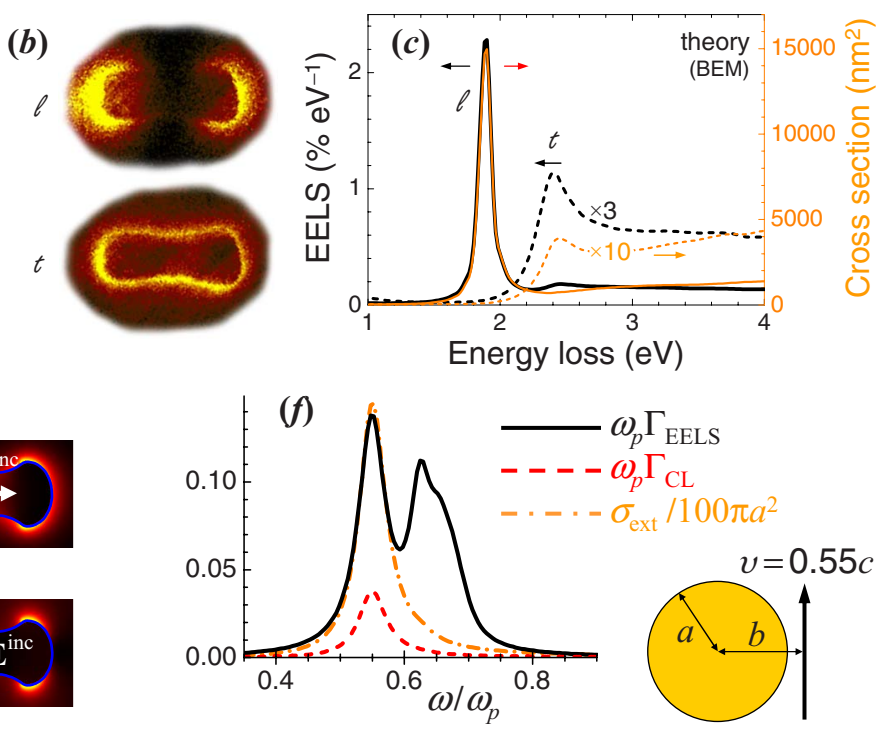

FIG. 36. (Color online) Comparison between EELS, CL, and optical extinction spectroscopy. (a) Electron energy-loss spectrum (EELS) of the dumbbell gold nanoparticle shown in the dark-field TEM image inset. (b) Plasmon maps obtained from the intensity of electrons that have lost an energy corresponding to the dipolar longitudinal mode $(1.7 \mathrm{eV})$ and the dipolar transverse mode $(2.4 \mathrm{eV})$. (c) Calculated EELS probability (left scale) and extinction cross section (right scale) of a nanoparticle mimicking the dumbbell of (a). The cross section is obtained for external electric field orientations both parallel and perpendicular to the particle axis in order to excite longitudinal $(l)$ and transversal $(t)$ plasmons, respectively. The EELS spectra correspond to positions of the electron beam as shown in (d) by circles. The longitudinal mode is redshifted in the experiment, as compared to the calculation, presumably due to the effect of a mica substrate, which is not accounted for here. (d) Calculated EELS plasmon maps, with the particle contour shown as a shaded area. Notice that the experimental images of (b) exhibit depletion of electron intensity for trajectories passing through the particle due to strong elastic scattering of the electrons with gold atoms. (e) Near-field plots for longitudinal and transversal polarizations of the incident electric field under external optical illumination. (f) Comparison of EELS and CL probabilities (solid and dashed curves, respectively) with the optical extinction cross section $\sigma_{\text {ext }}$ (dash-dotted curve) for a metal sphere described by the dielectric function of Eq. (A1). The damping rate is $\eta=0.04 \omega_{p}$ (similar to Al), the sphere radius is one-tenth of the free-space wavelength at frequency $\omega_{p}$, and the impact parameter is $b=1.1 a$. The experimental data of (a) and (b) are taken from Bosman et al., 2007. The calculations of (c)-(e) are adapted from Myroshnychenko, Rodríguez-Fernández, et al., 2008.

technique cannot provide details on the local distribution of these modes.

Spatially resolved EELS and CL offer alternative methods to characterize plasmons with unmatched resolution. Actually, no other available technique has achieved the degree of detail obtained by the plasmon studies that we show in Figs. 16, 28, and 36. Furthermore, the theoretical tools that are necessary for interpreting EELS and CL spectral images have already reached a mature status that allows addressing arbitrarily complex geometries (see Sec. III.E).

The plasmon energies and widths obtained from EELS and optical extinction are in good agreement (Fig. 36). In particular, the spectra of dumbbells that we show in Fig. 36(c) provide similar information in both techniques. However, EELS adds the spatial resolution that allows mapping of plasmons in real space [see Figs. 16 and 36(d)]. The resulting EELS images compare reasonably well with the near-field enhancement that is expected upon conventional optical illumination [Fig. 36(e)]. Moreover, EELS can access plasmon modes that are not resolvable with light (Chu et al., 2009; Koh et al., 2009), for example, multipoles of order $l>1$ in small metal spheres [Fig. 36(f)].

Like far-field optical spectroscopy, the EELS and CL techniques benefit from the use of relatively noninvasive probes (the electrons) that produce negligible changes in the optical properties of what is being observed. In contrast, the NSOM tip exerts a strong perturbation on the sample. The spatial resolution of EELS and CL is now pushed down to the subangstrom level, but even a modest SEM setup equipped with CL can focus an electron beam down to $<2 \mathrm{~nm}$ spots. The photon-energy resolution of CL is similar to that of other optical techniques, although the signal-to-noise ratio becomes annoying, particularly when the electron does not penetrate the specimen but simply interacts through electromagnetic fields at a short distance from it (aloof geometry). Regarding EELS, the $0.1 \mathrm{eV}$ resolution achieved by cutting-edge commercial STEMs is already sufficient for most plasmonic structures, and this situation should improve in coming years with further advances in electron monochromators and spectrometers.

Finally, it would be unfair to conclude this section without a summary of the impressive amount of information that has been gathered on both localized and extended plasmons using electron microscopes. We cannot review the vast literature here, but we present a 
TABLE I. Historical overview of experimental surface plasmon studies making use of electron beams.

\begin{tabular}{|c|c|c|c|c|c|}
\hline & Structure & Technique & $\begin{array}{c}\hbar \omega \\
(\mathrm{eV})\end{array}$ & Reference & Comments \\
\hline SP & $\mathrm{Al}$ planar surfaces & REELS & $5-100$ & Powell and Swan, 1959 & Observation of planar SPs \\
\hline SPP & $\mathrm{Al}$ gratings & $\mathrm{CL}$ & 2.3 & Teng and Stern, 1967 & SPP signature in $\mathrm{CL}$ \\
\hline LPR & Al nanoparticles & CL & $3-8$ & Fujimoto and Komaki, 1968 & Mie modes \\
\hline SPP & Al films & EELS & $2-12$ & Pettit et al., 1975 & SPP dispersion \\
\hline $\mathrm{SP}$ & $\mathrm{Al}$ and $\mathrm{In}$ surfaces & EELS & $8-12$ & Krane and Raether, 1976 & Large- $q$ dispersion \\
\hline SPP & Ag shallow grating & CL & $2-3$ & Heitmann, 1977 & SPP dispersion \\
\hline LPR & Al nanospheres & EELS & $1-20$ & Batson, 1980 & Size dependence of LPR \\
\hline LPR & $\mathrm{Al}$ spheres on $\mathrm{AlO}_{3}$ & EELS & $1-40$ & Wang and Cowley, 1987a & Plasmon maps \\
\hline LPR & Al spheres on $\mathrm{Al}$ & EELS & $1-40$ & Wang and Cowley, 1987a & Plasmon maps \\
\hline LPR & Ag nanospheres & EELS & $3-5$ & Ouyang et al., 1992 & Nonlocal effects \\
\hline LPR & Si nanospheres & EELS & $2-35$ & Ugarte et al., 1992 & Plasmon maps \\
\hline LPR & Al nanospheres & EELS & $2-40$ & Stöckli et al., 1997 & \\
\hline SP & $\mathrm{Al}_{2} \mathrm{O}_{3}$ nanospheres & EELS & $2-30$ & Abe et al., 2000 & Radiation losses \\
\hline SPP & Ag gratings & CL & $1-4$ & Yamamoto, Araya, Toda, and Sugiyama, 2001 & Plasmon standing waves \\
\hline LPR & Ag nanospheres & CL & $2-4$ & Yamamoto, Araya, and García de Abajo, 2001 & Plasmon maps of Mie modes \\
\hline LPR & $\mathrm{WS}_{3}$ nanotubes & EELS & $3-30$ & Kociak et al., 2001 & \\
\hline LPR & C nanotubes & EELS & $3-30$ & Stéphan et al., 2002 & $\pi$ and $\sigma$ plasmons \\
\hline LPR & Ag rough gratings & PEEM & 3 & Kubo et al., 2005 & Femtosecond dynamics \\
\hline SPP & Au planar surfaces & CL & $1-3$ & $\begin{array}{l}\text { Bashevoy et al., } 2006 \\
\text { van Wijngaarden et al., } 2006\end{array}$ & SPP propagation \\
\hline LPR & Ag sphere dimer & $\mathrm{CL}$ & $2-4$ & Yamamoto, Nakano, and Suzuki, 2006 & Plasmon maps \\
\hline LPR & Ag nanowires & CL & $1-4$ & Yamamoto, Nakano, and Suzuki, 2006 & Plasmon maps \\
\hline SPP & Au atom wire array & LEEM & $0.1-0.8$ & Nagao et al., 2006 & 1D plasmon dispersion \\
\hline LPR & Ag nanotriangles & EELS & $1-4$ & Nelayah, Kociak, et al., 2007 & Plasmon maps \\
\hline LPR & Ag nanowires & $\mathrm{CL}$ & $1-4$ & Vesseur et al., 2007 & Plasmon maps \\
\hline SPP & Au planar surfaces & $\mathrm{CL}$ & $1-3$ & Bashevoy et al., 2007 & Hyperspectral imaging \\
\hline SPP & Ag steps & PEEM & 3 & Kubo et al., 2007 & Femtosecond dynamics \\
\hline LPR & Au annular grating & CL & $1-3$ & Hofmann et al., 2007 & Plasmon maps \\
\hline $\mathrm{SP}$ & $\mathrm{HfO}_{2}$ in stacks & EELS & $2-50$ & Couillard et al., 2007 & Multilayer plasmons \\
\hline SP & $\mathrm{Si} / \mathrm{SiO}_{2} / \mathrm{Si}$ stacks & EELS & $2-50$ & Couillard et al., 2008 & \\
\hline SPP & Au planar surfaces & CL & $1-3$ & $\begin{array}{l}\text { Kuttge et al., } 2008 \\
\text { Peale et al., } 2008 \\
\text { Yamamoto and Suzuki, } 2008\end{array}$ & SPP propagation \\
\hline SPP & Au structured surfaces & CL & $1-3$ & Kuttge, Vesseur, and Polman, 2009 & SPP confinement \\
\hline LPR & $\mathrm{Au}$ ridge & CL & $1-3$ & Vesseur et al., 2008 & Plasmon maps \\
\hline LPR & $\begin{array}{l}\text { Au rods, spheres, } \\
\text { ellipsoids, dimers, } \\
\text { and touching dimers }\end{array}$ & EELS & $1-4$ & $\begin{array}{l}\text { Bosman et al., } 2007 \\
\text { N'Gom et al., } 2008 \\
\text { Chu et al., } 2009 \\
\text { Schaffer et al., } 2009\end{array}$ & Plasmon maps and spectra \\
\hline
\end{tabular}

succinct overview with some relevant references in Table I.

\section{CONCLUSION}

Overall, valence excitations, probed by electron microscopes, have been a continuous source of information on electronic structure and optical response of materials on the nanometer scale over the last six decades. The energetic electrons employed in these microscopes per- mit structural and spectroscopic details of the investigated samples to be obtained with subnanometer resolution, and they can be regarded as relatively contactless probes compared to other techniques such as STM of NSOM, particularly when the aloof configuration is employed, in which the electron beam passes outside the targeted materials and interacts with them via electromagnetic coupling. Optical excitations are the most intense in the energy-loss spectra, so that they can be collected using low beam currents, and therefore sample 
TABLE II. Dielectric parameters of selected materials, described by Eqs. (A1), (A3), and (A8) and chosen to fit the measured dielectric functions reported in the following references within the specified light-energy range: (a) Johnson and Christy, 1972; (b) Palik, 1985. The reader is referred to Raether (1980) for comprehensive tables of bulk and surface plasmon energies and widths.

\begin{tabular}{lcccccc}
\hline \hline Material & Equation & $\begin{array}{c}\hbar \omega_{p} \text { or } \hbar \omega_{\text {lon }} \\
(\mathrm{eV})\end{array}$ & $\begin{array}{c}\hbar \eta \\
(\mathrm{eV})\end{array}$ & $\begin{array}{c}\hbar \omega_{\mathrm{tr}} \text { or } \epsilon_{\infty} \\
(\mathrm{eV})\end{array}$ & $\begin{array}{c}\text { Energy range } \\
(\mathrm{eV})\end{array}$ \\
\hline $\mathrm{Ag}(\mathrm{a})$ & $(\mathrm{A} 8)$ & $9.17 \pm 0.19$ & $0.025 \pm 0.01$ & $\approx 4$ & & $<1.8$ \\
$\mathrm{Al}(\mathrm{b})$ & $(\mathrm{A} 1)$ & $\approx 15$ & $\approx 0.6$ & & $8-25$ \\
$\mathrm{Au} \mathrm{(a)}$ & $(\mathrm{A} 8)$ & $9.06 \pm 0.18$ & $0.071 \pm 0.007$ & $\approx 9.5$ & & $<1.8$ \\
$\mathrm{Cu}(\mathrm{a})$ & $(\mathrm{A} 8)$ & $8.88 \pm 0.18$ & $0.096 \pm 0.01$ & $\approx 8$ & & $<1.8$ \\
$\mathrm{KCl}(\mathrm{b})$ & $(\mathrm{A} 3)$ & $\approx 0.0244$ & $\approx 0.0008$ & $\approx 2.19$ & $\approx 0.0174$ & $0.01-0.025$ \\
$\mathrm{SiC}(\mathrm{b})$ & $(\mathrm{A} 3)$ & $\approx 0.124$ & $\approx 0.001$ & $\approx 6.15$ & $\approx 0.0985$ & $0.01-0.2$ \\
\hline \hline
\end{tabular}

damage is reduced to small levels even for penetrating trajectories. However, electron microscopes are versatile instruments in which the beam current can reach doses that cause substantial changes in the specimen, including reshaping of the morphology of metallic nanoparticles (Batson, 2008).

In the context of electron microscopy, CL constitutes an inexpensive addition to electron microscopes that can be valuable when combined with appropriate theoretical tools, the versatility of which we have tried to illustrate in this review. Electron microscopes in scanning mode of operation, combined with either EELS or CL analysis, are suitable tools for studying the optical response of nanostructures, and no other existing technique can match them in combined space-energy resolution. Furthermore, these instruments should facilitate the eventual application of swift electrons to light and plasmon sources for nanoscale devices. Finally, there is a large background of both experimental and theoretical expertise that constitutes a valuable guide for future research making use of electron microscopes in nanophotonics studies.

\section{ACKNOWLEDGMENTS}

It is a pleasure to express my gratitude to Archie Howie for countless generous suggestions and to Benito Hombrados Madrid and Carmen Martín Nieto for their kind technical assistance. I would also like to acknowledge many enjoyable and stimulating discussions with Harry A. Atwater, Cheng-Hsuan Chen, Ming-Wen Chu, Christian Colliex, Pedro M. Echenique, Archie Howie, Mathieu Kociak, Martin Kuttge, Viktor Myroshnychenko, Albert Polman, Alberto Rivacoba, Peter Schattschneider, Naoki Yamamoto, Nerea Zabala, and Nikolay Zheludev. This work was supported in part by the Spanish MICINN (Grant No. MAT2007-66050 and Consolider NanoLight.es) and by the EU (Grants No. NMP4-2006-016881 "SPANS" and No. NMP4-SL-2008213669 "ENSEMBLE").

\section{APPENDIX A: DIELECTRIC RESPONSE OF HOMOGENEOUS MEDIA}

The local response approximation yields good results when comparing theory and experiment both in the context of nanophotonics and in numerous EELS and CL studies that focus on the role of sample geometry. The different materials forming a structure are then described by frequency-dependent dielectric functions, usually taken from tabulated optical data (see, for example, Palik, 1985).

Several metals, including some alkali elements and aluminum, are remarkably well described by a freeelectron gas model, in which the local dielectric function takes the form ${ }^{28}$

$$
\epsilon^{D}(\omega)=1-\frac{\omega_{p}^{2}}{\omega(\omega+i \eta)}
$$

where

$$
\omega_{p}=\left(4 \pi e^{2} n_{0} / m_{e}\right)^{1 / 2}
$$

is the bulk plasmon frequency, determined by the conduction electron density $n_{0},{ }^{29}$ and $\eta$ is a small phenomenological relaxation rate that accounts for electromagnetic damping in the material (e.g., $\hbar \eta=0.6 \mathrm{eV}$ in $\mathrm{Al}$ ). This model gives $\hbar \omega_{p}=15.8 \mathrm{eV}$ for $\mathrm{Al}$, in good agreement with the value of $15 \mathrm{eV}$ obtained from EELS measurements (see Fig. 8 and Table II).

Some ionic materials and alkali-metal halide crystals in particular are better described by assuming localized ionic oscillation modes, which result in the dielectric function (Ashcroft and Mermin, 1976)

\footnotetext{
${ }^{28}$ Equation (A1) describes the correct way of incorporating damping via $\omega(\omega+i \eta)$ rather than $(\omega+i \eta)^{2}$. The latter is inconsistent with conservation of the number of electrons in the metal (Mermin, 1970).

${ }^{29}$ The dependence of $\omega_{p}$ on $n_{0}$ has been experimentally monitored by Imbusch and Niedrig (1970) using EELS and changing $n_{0}$ through thermal expansion in the 3-295 $\mathrm{K}$ range.
} 


$$
\epsilon(\omega)=\epsilon_{\infty}\left(1-\frac{\omega_{\text {lon }}^{2}-\omega_{\mathrm{tr}}^{2}}{\omega(\omega+i \eta)-\omega_{\mathrm{tr}}^{2}}\right)
$$

where $\omega_{\text {tr }}$ is the frequency of transverse vibrations and $\omega_{\text {lon }}$ signals a plasmon like longitudinal mode characterized by $\epsilon \approx 0$. Retardation produces dispersion of these modes for momentum transfers comparable to $\hbar k$, as shown in Fig. 5.

Neglecting the influence of the atomic lattice, the ratio between components of the external potential and the total potential (resolved in momentum and frequency) defines in general a dispersive dielectric function $\epsilon(q, \omega)$. Spatial dispersion becomes important for wave-vector transfers $q$ of the order of $\omega / v_{F}$, where the Fermi velocity $v_{F}$ is typically two or three orders of magnitude smaller than the speed of light $c$. Consequently, the dependence on $q$ is typically important for $q \gg k$ away from the region showing retardation [see Fig. 8(b)].

\section{Lindhard and Mermin dielectric functions}

Spatial dispersion effects in good metals such as aluminum and silver can be accounted for by assimilating their conduction band to a gas of noninteracting free electrons (Ashcroft and Mermin, 1976). An analytical formula for $\epsilon(q, \omega)$ in the free-electron gas was derived by Lindhard (1954). The Lindhard formula, which predicts a continuum of $e-h$ pair excitations and collective plasmon modes as shown in Fig. 8(a), is obtained by perturbing the plane wave representing each electron within the Fermi sphere by means of an external potential of the form $\phi^{\text {ext }}=\exp (i \mathbf{q} \cdot \mathbf{r}-i \omega t)$. The sum of these perturbations for all conduction electrons produces an induced charge $\rho^{\text {ind }}$ proportional to $\phi^{\text {ext }}$. The system is then assumed to be perturbed by the self-consistent total potential rather than the external potential in what has been termed the random-phase approximation (RPA). This leads to (Ashcroft and Mermin, 1976)

$$
\begin{aligned}
\epsilon(q, \omega) & =\left(1+\frac{4 \pi}{q^{2}} \frac{\rho^{\text {ind }}}{\phi^{\text {ext }}}\right)^{-1} \\
& =1+\frac{e^{2}}{\pi^{2} \hbar q^{2}} \int d^{3} \mathbf{k} \frac{f_{\mathbf{k}-\mathbf{q} / 2}-f_{\mathbf{k}+\mathbf{q} / 2}}{\hbar \mathbf{k} \cdot \mathbf{q} / m_{e}+\omega},
\end{aligned}
$$

where the integral extends over 3D electron wave vectors $\mathbf{k}, f_{k}$ is the Fermi distribution (approximately 1 for $k \leqslant k_{F}$ and 0 otherwise at room temperature), and $k_{F}$ $=m_{e} v_{F} / \hbar$ is the Fermi wave vector. This integral admits the closed-form expression (Lindhard, 1954)

$$
\epsilon^{\mathrm{L}}(q, \omega)=1+\frac{2 m_{e} e^{2} k_{F}}{\pi \hbar^{2} q^{2}}[1+R(x, y)+R(x,-y)],
$$

where $x=q / k_{F}, y=\omega / \varepsilon_{F}, \hbar \varepsilon_{F}=\hbar^{2} k_{F}^{2} / 2 m_{e}$ is the Fermi energy, and

$$
R(x, y)=\frac{1}{2 x}\left[1-\left(\frac{x^{2}+y}{2 x}\right)^{2}\right] \ln \left(\frac{x^{2}+2 x+y}{x^{2}-2 x+y}\right) .
$$

Here $\omega$ is assumed to have a positive imaginary part. If $\operatorname{Im}\{\omega\}$ is infinitesimally small then $\operatorname{Im}\left\{\epsilon^{\mathrm{L}}(q, \omega)\right\}$ takes nonzero values only for $x(x-2) \leqslant y \leqslant x(x+2)$ (i.e., in the so-called $e-h$ region), which corresponds to the shaded area of Fig. 8(a).

A naive way of introducing a damping rate $\eta$ in the electron-gas motion consists in adding a finite imaginary part to $\omega$, but this results in a net loss of the number of electrons with time. The Lindhard formula was extended by Mermin (1970) to include finite damping while maintaining the number of electrons constant. He found the expression

$$
\epsilon^{\mathrm{M}}(q, \omega)=1+\frac{(\omega+i \eta)\left[\epsilon^{\mathrm{L}}(q, \omega+i \eta)-1\right]}{\omega+i \eta\left[\epsilon^{\mathrm{L}}(q, \omega+i \eta)-1\right] /\left[\epsilon^{\mathrm{L}}(q, 0)-1\right]},
$$

where

$$
\epsilon^{\mathrm{L}}(q, 0)=1+\frac{2 m_{e} e^{2} k_{F}}{\pi \hbar^{2} q^{2}}\left(1+\frac{4-x^{2}}{4 x} \ln \left|\frac{x+2}{x-2}\right|\right)
$$

is the static limit of $\epsilon^{\mathrm{L}}$.

\section{Hydrodynamic model}

A simpler approach, which can be extended to nonlocal structured metals, consists in describing the conduction band as a classical electron plasma (Bloch, 1933). We generalize this approach here to incorporate retardation effects. The force equation for the hydrodynamic flow takes the form ${ }^{30}$

$$
\begin{aligned}
(\partial / \partial t+\eta) \mathbf{j}^{\text {ind }}= & \left(e^{2} / m_{e}\right) n_{0} \mathbf{E} \\
& -\left(\hbar / m_{e}\right)^{2}\left[\beta^{2}-(1 / 4) \nabla^{2}\right] \boldsymbol{\nabla} \rho^{\text {ind }},
\end{aligned}
$$

where $\rho^{\text {ind }}$ and $\mathbf{j}^{\text {ind }}$ are the charge density and current density perturbations induced in the electron gas, $\mathbf{E}$ is the self-consistent electric field, $n_{0}$ is the unperturbed electron density, and the hydrodynamic pressure is related to the Fermi wave vector $k_{F}$ through the coefficient $\beta$. Here we use the value $\beta=(3 / 5)^{1 / 2} k_{F}$, obtained from the large- $\omega$ limit of the Lindhard dielectric function (Pines and Bohm, 1952; Ritchie, 1957). This has to be compared to the classical plasma pressure $\beta^{2}$ $=\left(3 \hbar^{3} \pi^{2} n_{0}\right)^{2 / 3}\left(3 m_{e}\right)^{-1}$. We have supplemented this equation with the cutoff term $\left(\hbar / m_{e}\right)^{2}(1 / 4) \nabla^{2} \nabla \rho^{\text {ind }}$, which accounts for $e-h$ pair excitations in an effective way. The response of the system is then obtained by combining Eq. (A6) with Maxwell's equations for the total charge and current $\left(\rho^{\text {ext }}+\rho^{\text {ind }}\right.$ and $\mathbf{j}^{\text {ext }}+\mathbf{j}^{\text {ind }}$, respectively) in the presence of the ionic background, the response of which is accounted for through a permittivity $\epsilon_{b}$.'

\footnotetext{
${ }^{30}$ The linearized Bloch hydrodynamic equations constitute a suitable approximation within linear response under the assumption $\left|\rho^{\text {ind }}\right| \ll e n_{0}$.
} 
This formalism is a direct generalization of the common nonretarded approach that is based on Poisson's equation and expresses the current density through a velocity potential $\xi$ as $\mathbf{j}^{\text {ind }}=n_{0} \boldsymbol{\nabla} \xi$ [see, for instance, Ritchie (1957) for application to planar surfaces, Fujimoto and Komaki (1968), Crowell and Ritchie (1968), and Barberán and Bausells (1985) for spheres, and Wang and Cowley (1987a) for supported particles]. The customary boundary conditions of the electromagnetic field at the metal surface must now be supplemented by the condition that the normal component of $\mathbf{j}^{\text {ind }}$ is zero. The results of this formalism for planar surfaces and spheres are presented in Appendixes B.1 and $\mathrm{C}$, respectively.

In homogeneous media, it is convenient to project the hydrodynamic and Maxwell equations in the space of wave vector $\mathbf{q}$ and frequency $\omega$, from which we can work out the longitudinal and transversal dielectric functions $\epsilon_{\mathrm{lon}}$ and $\epsilon_{\mathrm{tr}}$ using the definition of electric displacement

$$
\mathbf{D}=\epsilon_{\mathrm{lon}} \mathbf{E}_{\mathrm{lon}}+\epsilon_{\mathrm{tr}} \mathbf{E}_{\mathrm{tr}}
$$

in terms of electric field components parallel and perpendicular to $\mathbf{q}$, respectively. We obtain

$$
\epsilon_{\text {lon }}(q, \omega)=\epsilon_{b}+\frac{\omega_{p}^{2}}{\left(\hbar / m_{e}\right)^{2}\left(\beta^{2} q^{2}+q^{4} / 4\right)-\omega(\omega+i \eta)}
$$

and

$$
\epsilon_{\mathrm{tr}}(q, \omega)=\epsilon_{b}-\frac{\omega_{p}^{2}}{\omega(\omega+i \eta)} .
$$

Equation (A7) was derived by Ritchie (1957) from an expansion of Lindhard's function for large $\omega$. The dispersion relation of longitudinal and transversal bulk plasmons is shown in Fig. 8(b) for aluminum.

\section{APPENDIX B: NONRETARDED SCREENED INTERACTION IN SIMPLE GEOMETRIES}

The electrostatic screened interaction $W\left(\mathbf{r}, \mathbf{r}^{\prime}, \omega\right)$ is defined as the amplitude of the potential created at $\mathbf{r}$ by a charge of magnitude exp $(-i \omega t)$ placed at $\mathbf{r}^{\prime}$. For materials described by a local frequency-dependent dielectric function $\epsilon(\mathbf{r}, \omega)$, Poisson's equation leads to

$$
\boldsymbol{\nabla} \cdot \boldsymbol{\epsilon}(\mathbf{r}, \omega) \boldsymbol{\nabla} W\left(\mathbf{r}, \mathbf{r}^{\prime}, \omega\right)=-4 \pi \delta\left(\mathbf{r}-\mathbf{r}^{\prime}\right) .
$$

In free space, one has $W^{0}\left(\mathbf{r}-\mathbf{r}^{\prime}\right)=\left|\mathbf{r}-\mathbf{r}^{\prime}\right|^{-1}$. While Eq. (B1) is valid for arbitrary smooth spatial dependence of the permittivity, most situations encountered in practice are reliably modeled by sharp boundaries. We can then obtain $W$ from the Coulomb interaction in each homogeneous region after imposing the continuity of both the potential and the normal displacement at the interfaces. Next, we offer analytical expressions for $W$ in the simple cases of planes, cylinders, and spheres.

\section{Planar surface}

A planar interface at $z=0$ is best described by expressing $W$ in the space of parallel wave vector $\mathbf{q}_{\|}$. In particular, the bare Coulomb interaction can be written as

$$
\frac{1}{\left|\mathbf{r}-\mathbf{r}^{\prime}\right|}=\int \frac{d^{2} \mathbf{q}_{\|}}{(2 \pi)^{2}} e^{i \mathbf{q}_{\|}\left(\mathbf{R}-\mathbf{R}^{\prime}\right)} W^{0}\left(q_{\|}, z, z^{\prime}\right),
$$

where $\mathbf{r}=(\mathbf{R}, z)$ and

$$
W^{0}\left(q_{\|}, z, z^{\prime}\right)=\frac{2 \pi}{q_{\|}} e^{-q_{\|}\left|z-z^{\prime}\right|} .
$$

The full screened interaction $W$ admits an expansion similar to Eq. (B2), in which $W^{0}\left(q_{\|}, z, z^{\prime}\right)$ must be replaced by

$$
W\left(q_{\|,}, z, z^{\prime}, \omega\right)=\frac{2 \pi}{q_{\|}} \times \begin{cases}\frac{1}{\epsilon_{1}}\left(e^{-q_{\|}\left|z-z^{\prime}\right|}+\frac{\epsilon_{1}-\epsilon_{2}}{\epsilon_{1}+\epsilon_{2}} e^{-q_{\|}\left(z+z^{\prime}\right)}\right), & z, z^{\prime}>0 \\ \frac{1}{\epsilon_{2}}\left(e^{-q_{\|}\left|z-z^{\prime}\right|}-\frac{\epsilon_{1}-\epsilon_{2}}{\epsilon_{1}+\epsilon_{2}} e^{q\left(\mid\left(z+z^{\prime}\right)\right.}\right), & z, z^{\prime}<0 \\ \frac{2}{\epsilon_{1}+\epsilon_{2}} e^{-q_{\|} \mid\left(z|+| z^{\prime} \mid\right)} & \text { otherwise }\end{cases}
$$

using the notation of Fig. 9. ${ }^{3132}$

Nonlocal effects can be incorporated through the hydrodynamic model outlined in Appendix A.2. The result in the nonretarded approximation reduces to

\footnotetext{
${ }^{31}$ Interband transitions in aluminum give the main contribution to $\eta$, but $\epsilon_{b}=1$ works well for that material, indicating that they are not strongly polarized near the bulk plasmon frequency (Raether, 1980). In contrast, interband transitions play a dominant role in noble metals such as $\mathrm{Ag}, \mathrm{Au}$, and $\mathrm{Cu}$ (Johnson and Christy, 1972), where $\epsilon_{b}$ differs considerably from 1 (see Table II),

${ }^{32}$ Notice that the continuity equation $\nabla \cdot \mathbf{j}=i \omega \rho$ is implied by Maxwell's equations.
} 


$$
W\left(q_{\|}, z, z^{\prime}, \omega\right)=\frac{2 \pi}{q_{\|}} \times \begin{cases}e^{-q \| z-z^{\prime} \mid}+\frac{\epsilon_{s}(0)-1}{\epsilon_{s}(0)+1} e^{-q \|\left(z+z^{\prime}\right)}, & z, z^{\prime} \geqslant 0 \\ \epsilon_{s}\left(z-z^{\prime}\right)+\epsilon_{s}\left(z+z^{\prime}\right)-\frac{2 \epsilon_{s}(z) \epsilon_{s}\left(z^{\prime}\right)}{\epsilon_{s}(0)+1}, & z, z^{\prime}<0 \\ \frac{2 \epsilon_{s}\left(z^{\prime}\right)}{\epsilon_{s}(0)+1} e^{-q \|^{z}}, & z \geqslant 0, z^{\prime}<0 \\ \frac{2 \epsilon_{s}(z)}{\epsilon_{s}(0)+1} e^{-q \| z^{\prime}}, & z<0, z^{\prime} \geqslant 0,\end{cases}
$$

where

$$
\epsilon_{s}(z)=\frac{q_{\|}}{\pi} \int \frac{d q_{z}}{q^{2}} \frac{e^{i q_{z} z}}{\epsilon_{\mathrm{lon}}(q, \omega)}
$$

and $q^{2}=q_{\|}^{2}+q_{z}^{2}$. An analytical expression for $\epsilon_{s}(z)$ can be obtained using Eq. (A7) (García de Abajo and Echenique, 1992). This approach is formally equivalent to the specular reflection model (Ritchie and Marusak, 1966; Wagner, 1966), which applies to any $\epsilon_{\text {lon }}(q, \omega)$.

\section{Cylinder}

For axially symmetric problems, it is convenient to write the bare Coulomb interaction as

$$
\frac{1}{\left|\mathbf{r}-\mathbf{r}^{\prime}\right|}=\frac{1}{(2 \pi)^{2}} \int_{-\infty}^{\infty} d q_{z} e^{i q_{z}\left(z-z^{\prime}\right)} \sum_{m=-\infty}^{\infty} e^{i m\left(\varphi-\varphi^{\prime}\right)} W_{m}^{0}\left(R, R^{\prime}, q_{z}, \omega\right),
$$

where

$$
W_{m}^{0}\left(R, R^{\prime}, q_{z}\right)=4 \pi I_{m}\left(q_{z} R_{<}\right) K_{m}\left(q_{z} R_{>}\right)
$$

$R_{<}=\min \left\{R, R^{\prime}\right\}$, and $R_{>}=\max \left\{R, R^{\prime}\right\}$. In a homogeneous cylinder centered around the $z$ axis (see Fig. 13), the screened interaction is found to be

$$
W_{m}\left(R, R^{\prime}, q_{z}, \omega\right)=4 \pi \times \begin{cases}\frac{1}{\epsilon_{1}}\left[I_{m}\left(q_{z} R_{<}\right)+\frac{1}{\Delta_{m}}\left(\epsilon_{1}-\epsilon_{2}\right) I_{m}(x) I_{m}^{\prime}(x) K_{m}\left(q_{z} R_{<}\right)\right] K_{m}\left(q_{z} R_{>}\right) & \text {if } R, R^{\prime}>a \\ \frac{1}{\epsilon_{2}}\left[K_{m}\left(q_{z} R_{>}\right)+\frac{1}{\Delta_{m}}\left(\epsilon_{1}-\epsilon_{2}\right) K_{m}(x) K_{m}^{\prime}(x) I_{m}\left(q_{z} R_{>}\right)\right] I_{m}\left(q_{z} R_{<}\right) & \text {if } R, R^{\prime}<a \\ \frac{1}{\Delta_{m} x} I_{m}\left(q_{z} R_{<}\right) K_{m}\left(q_{z} R_{>}\right) & \text {otherwise },\end{cases}
$$

where $\Delta_{m}=\epsilon_{2} I_{m}^{\prime}(x) K_{m}(x)-\epsilon_{1} I_{m}(x) K_{m}^{\prime}(x)$ and $x=q_{z} a$.

\section{Sphere}

The bare Coulomb interaction can be expanded in spherical harmonics as

$$
\frac{1}{\left|\mathbf{r}-\mathbf{r}^{\prime}\right|}=\sum_{l=0}^{\infty} \sum_{m=-l}^{m=l} \frac{4 \pi}{2 l+1} W_{l}^{0}\left(r, r^{\prime}\right) Y_{l m}(\Omega) Y_{l m}^{*}\left(\Omega^{\prime}\right),
$$

where

$$
W_{l}^{0}\left(r, r^{\prime}\right)=\frac{r_{<}^{l}}{r_{>}^{l+1}},
$$

$r_{<}=\min \left\{r, r^{\prime}\right\}, r_{>}=\max \left\{r, r^{\prime}\right\}$, and $\Omega$ denotes the direction of $\mathbf{r}$. Using the notation of Fig. 13, the interaction near a homogeneous sphere is given by the above expansion with

$$
W_{l}\left(r, r^{\prime}, \omega\right)
$$

$$
= \begin{cases}\frac{1}{\epsilon_{1}} W_{l}^{0}\left(r, r^{\prime}\right)+\left(\frac{2 l+1}{\eta_{l}}-\frac{1}{\epsilon_{1}}\right) \frac{a^{2 l+1}}{\left(r r^{\prime}\right)^{l+1}} & \text { if } r, r^{\prime}>a \\ \frac{1}{\epsilon_{2}} W_{l}^{0}\left(r, r^{\prime}\right)+\left(\frac{2 l+1}{\eta_{l}}-\frac{1}{\epsilon_{2}}\right) \frac{\left(r r^{\prime}\right)^{l}}{a^{2 l+1}} & \text { if } r, r^{\prime}<a \\ \frac{2 l+1}{\eta_{l}} W_{l}^{0}\left(r, r^{\prime}\right) & \text { otherwise, }\end{cases}
$$

substituted for $W_{l}^{0}\left(r, r^{\prime}\right)$. Here $\eta_{l}=(l+1) \epsilon_{1}+l \epsilon_{2}$. It is also useful to write the $r, r^{\prime}>a$ part in terms of the multipolar polarizability 
TABLE III. Analytical expressions of the coefficients $C_{l m}^{M}$ and $C_{l m}^{E}$ for $l \leqslant 4$. These coefficients are independent of the sign of $m$.

\begin{tabular}{|c|c|c|c|}
\hline$l$ & $m$ & $\pi(v / c)^{2 l} C_{l m}^{M}$ & $\pi(v / c)^{2 l+2} C_{l m}^{E}$ \\
\hline 1 & 0 & 0 & $6 / \gamma^{4}$ \\
\hline 1 & 1 & $3 / \gamma^{2}$ & $3 / \gamma^{2}$ \\
\hline 2 & 0 & 0 & $30 / \gamma^{4}$ \\
\hline 2 & 1 & $5 / \gamma^{2}$ & $\left(5 / \gamma^{6}\right)\left(1+\gamma^{2}\right)^{2}$ \\
\hline 2 & 2 & $5 / \gamma^{4}$ & $5 / \gamma^{4}$ \\
\hline 3 & 0 & 0 & $\left(21 / 4 \gamma^{8}\right)\left(1+4 \gamma^{2}\right)^{2}$ \\
\hline 3 & 1 & $\left(7 / 16 \gamma^{6}\right)\left(1+4 \gamma^{2}\right)^{2}$ & $\left(7 / 16 \gamma^{6}\right)\left(11+4 \gamma^{2}\right)^{2}$ \\
\hline 3 & 2 & $35 / 2 \gamma^{4}$ & $\left(35 / 8 \gamma^{8}\right)\left(1+2 \gamma^{2}\right)^{2}$ \\
\hline 3 & 3 & $105 / 16 \gamma^{6}$ & $105 / 16 \gamma^{6}$ \\
\hline 4 & 0 & 0 & $\left(45 / 4 \gamma^{8}\right)\left(3+4 \gamma^{2}\right)^{2}$ \\
\hline 4 & 1 & $\left(9 / 16 \gamma^{6}\right)\left(3+4 \gamma^{2}\right)^{2}$ & $\left(9 / 16 \gamma^{10}\right)\left(3+21 \gamma^{2}+4 \gamma^{4}\right)^{2}$ \\
\hline 4 & 2 & $\left(9 / 8 \gamma^{8}\right)\left(1+6 \gamma^{2}\right)^{2}$ & $\left(9 / 2 \gamma^{8}\right)\left(4+3 \gamma^{2}\right)^{2}$ \\
\hline 4 & 3 & $567 / 16 \gamma^{6}$ & $\left(63 / 16 \gamma^{10}\right)\left(1+3 \gamma^{2}\right)^{2}$ \\
\hline 4 & 4 & $63 / 8 \gamma^{8}$ & $63 / 8 \gamma^{8}$ \\
\hline
\end{tabular}

$$
\alpha_{l}^{\mathrm{NR}}(\omega)=a^{2 l+1} \frac{l\left(\epsilon_{2}-\epsilon_{1}\right)}{l \epsilon_{2}+(l+1) \epsilon_{1}}
$$

as

$$
W_{l}\left(r, r^{\prime}, \omega\right)=\frac{1}{\epsilon_{1}}\left(W_{l}^{0}\left(r, r^{\prime}\right)-\frac{\alpha_{l}^{\mathrm{NR}}}{\left(r r^{\prime}\right)^{l+1}}\right) .
$$

\section{APPENDIX C: RETARDED SCATTERING AND COUPLING COEFFICIENTS IN A SPHERE INCLUDING NONLOCAL EFFECTS}

The coupling coefficients of Eqs. (33) and (58) are dimensionless functions of $v / c$ that admit the following closed-form expressions:

$$
C_{l m}^{M}=\frac{1}{l(l+1)}\left|2 m N_{l m}\right|^{2}
$$

and

$$
C_{l m}^{E}=\frac{1}{l(l+1)}\left|\frac{c}{v \gamma} M_{l m}\right|^{2}
$$

where

$$
\begin{aligned}
N_{l m}= & \sqrt{\frac{(2 l+1)}{\pi} \frac{(l-|m|) !}{(l+|m|) !} \frac{(2|m|-1) ! !}{(v \gamma / c)^{|m|}} C_{l-|m|}^{(|m|+1 / 2)}\left(\frac{c}{v}\right),} \\
M_{l m}= & N_{l m+1} \sqrt{(l+m+1)(l-m)} \\
& +N_{l m-1} \sqrt{(l-m+1)(l+m)},
\end{aligned}
$$

and $C_{m}^{(\nu)}$ are Gegenbauer polynomials (Abramowitz and Stegun, 1972). Explicit analytical expressions of $C_{l m}^{M}$ and $C_{l m}^{E}$ are given in Table III for $l \leqslant 4$. Similar formulas are encountered to describe the Coulomb excitation of atomic nuclei (Winther and Alder, 1979).

The scattering coefficients of Eqs. (33) and (58) depend on the composition and structure of the spheres (see Table III). For the homogeneous particle of Fig. 13, Mie theory leads to (Mie, 1908; Jackson, 1999)

$$
t_{l}^{M}=\frac{-j_{l}\left(x_{1}\right) x_{2} j_{l}^{\prime}\left(x_{2}\right)+x_{1} j_{l}^{\prime}\left(x_{1}\right) j_{l}\left(x_{2}\right)}{h_{l}^{(+)}\left(x_{1}\right) x_{2} j_{l}^{\prime}\left(x_{2}\right)-x_{1}\left[h_{l}^{(+)}\left(x_{1}\right)\right]^{\prime} j_{l}\left(x_{2}\right)}
$$

and

$$
t_{l}^{E}=\frac{-\epsilon_{1} j_{l}\left(x_{1}\right)\left[x_{2} j_{l}\left(x_{2}\right)\right]^{\prime}+\epsilon_{2}\left[x_{1} j_{l}\left(x_{1}\right)\right]^{\prime} j_{l}\left(x_{2}\right)}{\epsilon_{1} h_{l}^{(+)}\left(x_{1}\right)\left[x_{2} j_{l}\left(x_{2}\right)\right]^{\prime}-\epsilon_{2}\left[x_{1} h_{l}^{(+)}\left(x_{1}\right)\right]^{\prime} j_{l}\left(x_{2}\right)},
$$

where $x_{1}=k a \sqrt{\epsilon_{1}}, x_{2}=k a \sqrt{\epsilon_{2}}$, the prime denotes differentiation with respect to the argument, and $j_{l}$ and $h_{l}^{(+)}=i j_{l}$ $-y_{l}$ are spherical Bessel and Hankel functions, respectively.

We can incorporate nonlocal effects in the sphere response using the formalism of Appendix A.2. This allows us to generalize the nonretarded study of dispersive spheres reported by Fujimoto and Komaki (1968). Considering a metallic sphere in vacuum [i.e., $\epsilon_{1}=1$ and with medium 2 inside the sphere described by $\epsilon_{\text {lon }}(q, \omega)$ and $\left.\epsilon_{\mathrm{tr}}(\omega)\right]$, a detailed analysis leads to

$$
t_{l}^{E}=\frac{-j_{l}(x)\left\{\left[x_{2} j_{l}\left(x_{2}\right)\right]^{\prime}+g_{l}\right\}+\epsilon_{\mathrm{tr}}\left[x j_{l}(x)\right]^{\prime} j_{l}\left(x_{2}\right)}{h_{l}^{(+)}(x)\left\{\left[x_{2} j_{l}\left(x_{2}\right)\right]^{\prime}+g_{l}\right\}-\epsilon_{\mathrm{tr}}\left[x h_{l}^{(+)}(x)\right]^{\prime} j_{l}\left(x_{2}\right)},
$$

where 


$$
g_{l}=\frac{l(l+1) j_{l}\left(x_{2}\right) j_{l}\left(q_{0} a\right)}{q_{0} a j_{l}^{\prime}\left(q_{0} a\right)}\left(\frac{\epsilon_{\mathrm{tr}}}{\epsilon_{b}}-1\right),
$$

$x=k a, x_{2}=k a \sqrt{\epsilon_{\mathrm{tr}}}$, and $q_{0}$ is given by the condition $\epsilon_{\text {lon }}\left(q_{0}, \omega\right)=0$ [see Eq. (A7)]. ${ }^{33}$ The magnetic scattering coefficients $t_{l}^{M}$ are still given by Eq. (C1) with $\epsilon_{\mathrm{tr}}$ [Eq. (A8)] substituted for $\epsilon_{2}$ and $\epsilon_{1}=0$.

\section{NOMENCLATURE}

\section{List of selected symbols}

$\begin{array}{lll}\mathbf{A} & \text { vector potential } & \epsilon_{\mathrm{lon}}, \epsilon_{\mathrm{tr}} \\ a & \text { sphere or cylinder radius } & \varepsilon \\ \mathbf{B} & \text { magnetic induction } & \eta \\ b & \text { electron impact parameter } & \lambda \\ c & \text { speed of light } & \mu_{j} \\ C_{l m}^{E}, C_{l m}^{M} & \text { electron-sphere coupling coefficients } & \rho \\ e & \text { minus the electron charge } & \rho_{\hat{\mathbf{n}}} \\ \mathbf{E} & \text { electric field } & \sigma \\ \mathbf{F} & \text { electromagnetic force } & \phi \\ \mathbf{f} & \text { far-field amplitude } & \hat{\varphi} \\ G & \text { electromagnetic Green's tensor } & \hat{\varphi}_{\mathbf{q}} \\ \mathcal{G}^{0} & \text { dipole-dipole interaction in vacuum } & \varphi \\ \mathbf{H} & \text { magnetic field } & \varphi_{\mathrm{in}} \\ \mathbf{h} & \text { boundary current } & \varphi_{\mathrm{out}} \\ \hbar & \text { Planck's constant } & \chi \\ h_{l}^{(+)} & \text {spherical Hankel function of order } l & \psi \\ I_{m} & \text { modified Bessel function of order } m & \Omega \\ \mathbf{j} & \text { electron current density } & \omega \\ j_{l} & \text { spherical Bessel function of order } l & \omega_{p} \\ k=\omega / c & \text { free-space light wave vector } & \omega_{s} \\ k_{B} & \text { Boltzmann's constant } & \end{array}$

electron velocity

$\begin{array}{ll}v_{F} & \text { Fermi velocity } \\ W & \text { scalar screened }\end{array}$

$W \quad$ scalar screened interaction

$\hat{\mathbf{x}}, \hat{\mathbf{y}}, \hat{\mathbf{z}} \quad$ Cartesian unit vectors

$\alpha \quad$ particle polarizability

$\beta \quad$ hydrodynamic wave vector

$\Gamma(\omega) \quad$ probability per unit frequency $\omega$

$\gamma \quad \gamma_{\epsilon}$ in vacuum $(\epsilon=1)$

$\gamma_{\epsilon} \quad$ Lorentz's contraction factor in a medium of permittivity $\epsilon$

$\epsilon \quad$ dielectric function

$\epsilon^{\mathrm{D}}, \epsilon^{\mathrm{L}}, \epsilon^{\mathrm{M}} \quad$ Drude, Lindhard, and Mermin $\epsilon$

longitudinal and transversal $\epsilon$

$\hbar \varepsilon$ electron energy

bulk plasmon damping rate

free-space light wavelength

coefficients for TR and SPP emission

electron charge density

photonic local density of states (LDOS)

boundary charge

scalar potential

azimuthal unit vector

azimuthal unit vector in $\mathbf{q}$ space, $\perp \mathbf{q}_{\|}, \hat{\mathbf{z}}$

azimuthal angle

electron-beam divergence half-angle

electron-spectrometer aperture half-angle

electric susceptibility

electron wave function

solid angle

frequency

classical bulk plasma frequency

Ritchie's surface plasma frequency

\section{List of selected acronyms}

$K_{m} \quad$ modified Bessel function of order $m$

$L \quad$ length of the electron trajectory

$l, m \quad$ orbital and azimuthal numbers

$l_{\max } \quad$ maximum orbital angular-momentum

number

$m_{e} \quad$ electron mass

$\mathbf{p}_{i}, \mathbf{p}_{f} \quad$ initial and final electron wave vectors

q wave vector transferred from the electron

$\mathbf{q}_{\|}=\left(q_{x}, q_{y}\right) \quad$ wave vector parallel to a planar surface

$\mathbf{q}_{\perp}$

wave-vector transfer perpendicular to $\mathbf{v}$

$R \quad$ radial distance in cylindrical coordinates

$\mathbf{R}=(x, y)$

$\hat{\mathbf{R}}$

in-plane or $\perp \hat{\mathbf{z}}$ components of $\mathbf{r}$

cylindrical radial unit vector

$r \quad$ radial distance in spherical coordinates

$\mathbf{r}=(\mathbf{R}, z) \quad$ position coordinate vector

$\mathbf{r}_{e}(t) \quad$ electron trajectory

$\mathbf{r}_{0}=\left(\mathbf{R}_{0}, z_{0}\right) \quad$ electron position at time $t=0$

$t$ time

$t_{l}^{E}, t_{l}^{M} \quad$ sphere scattering coefficients

$\mathbf{v}$ electron velocity vector

$\begin{array}{ll}\text { 1D,2D,3D } & \text { one-, two-, three-dimensional } \\ \text { ADF } & \text { annular dark field } \\ \text { BEM } & \text { boundary element method } \\ \text { CL } & \text { cathodoluminescence } \\ \text { CR } & \text { Cherenkov radiation } \\ \text { DDA } & \text { discrete-dipole approximation } \\ \text { DFM } & \text { dark-field microscopy } \\ \text { EEGS } & \text { electron energy-gain spectroscopy } \\ \text { EELS } & \text { electron energy-loss spectroscopy } \\ \text { EFTEM } & \text { energy-filtered TEM } \\ e-h & \text { electron-hole } \\ \text { EIRE } & \text { electron-induced radiation emission } \\ \text { FWHM } & \text { full width at half maximum } \\ \text { HREELS } & \text { high-resolution EELS } \\ \text { JDOS } & \text { joint density of states } \\ \text { KK } & \text { Kramers-Kronig } \\ \text { KKR } & \text { Korringa-Kohn-Rostoker } \\ \text { LDOS } & \text { local density of states } \\ \text { LEEM } & \text { low-energy electron microscopy } \\ \text { LPR } & \text { localized plasmon resonance } \\ \text { NIR } & \text { near infrared } \\ \text { NR } & \text { nonretarded } \\ \text { NSOM } & \text { near-field scanning optical microscopy }\end{array}$

NSOM near-field scanning optical microscopy 


$\begin{array}{ll}\text { PEEM } & \text { photoelectron emission microscopy } \\ \text { REELS } & \text { reflection EELS } \\ \text { SE } & \text { secondary electron } \\ \text { SEE } & \text { secondary electron emission } \\ \text { SEM } & \text { scanning electron microscopy (micro- } \\ & \text { scope) } \\ \text { SERS } & \text { surface-enhanced Raman scattering } \\ \text { SP } & \text { surface plasmon } \\ \text { SPP } & \text { surface-plasmon polariton } \\ \text { S-P } & \text { Smith-Purcell } \\ \text { STEM } & \text { scanning transmission electron microscopy } \\ & \text { (microscope) } \\ \text { STM } & \text { scanning tunneling microscopy } \\ \text { STS } & \text { scanning tunneling spectroscopy } \\ \text { TEM } & \text { transmission electron microscopy (micro- } \\ \text { TR } & \text { scope) } \\ \text { uv } & \text { transition radiation } \\ \text { XRM } & \text { ultraviolet } \\ \text { ZLP } & \text { x-ray microscopy } \\ & \text { zero-loss peak }\end{array}$

\section{REFERENCES}

Abe, H., H. Kurata, and K. Hojou, 2000, "Spatially resolved electron energy-loss spectroscopy of the surface excitations on the insulating fine particle of aluminum oxide," J. Phys. Soc. Jpn. 69, 1553-1557.

Abramowitz, M., and I. A. Stegun, 1972, Handbook of Mathematical Functions (Dover, New York).

Achèche, M., C. Colliex, H. Kohl, A. Nourtier, and P. Trebbia, 1986, "Theoretical and experimental study of plasmon excitations in small metallic spheres," Ultramicroscopy 20, 99106.

Adamo, G., K. F. MacDonald, Y. H. Fu, C. M. Wang, D. P. Tsai, F. J. García de Abajo, and N. I. Zheludev, 2009, "Light well: A tunable free-electron light source on a chip," Phys. Rev. Lett. 103, 113901.

Aeschlimann, M., M. Bauer, D. Bayer, T. Brixner, F. J. García de Abajo, W. Pfeiffer, M. Rohmer, C. Spindler, and F. Steeb, 2007, "Adaptive subwavelength control of nano-optical fields," Nature (London) 446, 301-304.

Ahn, C. C., 2004, Ed., Transmission Electron Energy Loss Spectrometry in Materials Science and the EELS Atlas (Wiley$\mathrm{VCH}$, Weinheim).

Aizpurua, J., P. Hanarp, D. S. Sutherland, M. Käll, G. W. Bryant, and F. J. García de Abajo, 2003, "Optical properties of gold nanorings," Phys. Rev. Lett. 90, 057401.

Aizpurua, J., A. Howie, and F. J. García de Abajo, 1999, "Valence-electron energy loss near edges, truncated slabs, and junctions," Phys. Rev. B 60, 11149-11162.

Aizpurua, J., and A. Rivacoba, 2008, "Nonlocal effects in the plasmons of nanowires and nanocavities excited by fast electron beams," Phys. Rev. B 78, 035404.

Aizpurua, J., A. Rivacoba, and S. P. Apell, 1996, "Electronenergy losses in hemispherical targets," Phys. Rev. B 54, 2901-2909.

Akhmediev, N., and M. Karlsson, 1995, "Cherenkov radiation emitted by solitons in optical fibers," Phys. Rev. A 51, 2602 2607.

Allen, L. J., S. D. Findlay, A. R. Lupini, M. P. Oxley, and S. J. Pennycook, 2003, "Atomic-resolution electron energy loss spectroscopy imaging in aberration corrected scanning trans- mission electron microscopy," Phys. Rev. Lett. 91, 105503.

Andrew, P., and W. L. Barnes, 2001, "Molecular fluorescence above metallic gratings," Phys. Rev. B 64, 125405.

Arakawa, E. T., R. J. Herickhoff, and R. D. Birkhoff, 1964, "Detection of plasma radiation from electron-bombarded Al and Mg foils," Phys. Rev. Lett. 12, 319-320.

Arenal, R., O. Stéphan, M. Kociak, D. Taverna, A. Loiseau, and C. Colliex, 2005, "Electron energy loss spectroscopy measurement of the optical gaps on individual boron nitride single-walled and multiwalled nanotubes," Phys. Rev. Lett. 95, 127601.

Arslan, I., J. K. Hyun, R. Erni, M. N. Fairchild, S. D. Hersee, and D. A. Muller, 2009, "Using electrons as a high-resolution probe of optical modes in individual nanowires," Nano Lett. 9, 4073-4077.

Ashcroft, N. W., and N. D. Mermin, 1976, Solid State Physics (Harcourt College, New York).

Ashkin, A., and J. M. Dziedzic, 1976, "Optical levitation in high vacuum," Appl. Phys. Lett. 28, 333-335.

Ashley, J. C., and L. C. Emerson, 1974, "Dispersion relations for non-radiative surface plasmons on cylinders," Surf. Sci. 41, 615-618.

Ashley, J. C., and T. L. Ferrell, 1976, "Excitation by fast electrons of surface plasmons on spherical voids in a metal," Phys. Rev. B 14, 3277-3281.

Atay, T., J. H. Song, and A. V. Nurmikko, 2004, "Strongly interacting plasmon nanoparticle pairs: From dipole-dipole interaction to conductively coupled regime," Nano Lett. 4, 1627-1631.

Averkov, Y. O., and V. M. Yakovenko, 2005, "Cherenkov radiation by an electron bunch that moves in a vacuum above a left-handed material," Phys. Rev. B 72, 205110.

Bachheimer, J. P., 1972, "Experimental investigation of the interaction radiation of a moving electron with a metallic grating: The Smith-Purcell effect," Phys. Rev. B 6, 2985-2994.

Bakunov, M. I., A. V. Maslov, and S. B. Bodrov, 2005, "Cherenkov radiation of terahertz surface plasmon polaritons from a superluminal optical spot," Phys. Rev. B 72, 195336.

Balberg, I., and J. I. Pankove, 1971, "Cathodoluminescence of magnetite,” Phys. Rev. Lett. 27, 1371-1374.

Barberán, N., and J. Bausells, 1985, "Plasmon excitation in metallic spheres," Phys. Rev. B 31, 6354-6359.

Barnes, W. L., A. Dereux, and T. W. Ebbesen, 2003, "Surface plasmon subwavelength optics," Nature (London) 424, 824830.

Barnett, S. M., and R. Loudon, 1996, "Sum rule for modified spontaneous emission rates,” Phys. Rev. Lett. 77, 2444-2446.

Barrera, R. G., and R. Fuchs, 1995, "Theory of electron energy loss in a random system of spheres," Phys. Rev. B 52, 32563273.

Barwick, B., D. J. Flannigan, and A. H. Zewail, 2009, "Photon induced near-field electron microscopy," Nature (London) 462, 902-906.

Barwick, B., H. S. Park, O. H. Kwon, J. S. Baskin, and A. H. Zewail, 2008, "4D Imaging of transient structures and morphologies in ultrafast electron microscopy," Science 322, 1227-1231.

Bashevoy, M. V., F. Jonsson, A. V. Krasavin, N. I. Zheludev, Y. Chen, and M. I. Stockman, 2006, "Generation of traveling surface plasmon waves by free-electron impact," Nano Lett. 6, 1113-1115.

Bashevoy, M. V., F. Jonsson, K. F. MacDonald, Y. Chen, and N. I. Zheludev, 2007, "Hyperspectral imaging of plasmonic 
nanostructures with nanoscale resolution," Opt. Express 15 11313-11320.

Batson, P. E., 1980, "Damping of bulk plasmons in small aluminum spheres," Solid State Commun. 34, 477-480.

Batson, P. E., 1982a, "A new surface plasmon resonance in clusters of small aluminum spheres," Ultramicroscopy 9, 277 282.

Batson, P. E., 1982b, "Surface plasmon coupling in clusters of small spheres," Phys. Rev. Lett. 49, 936-940.

Batson, P. E., 1985, "Inelastic scattering of fast electrons in clusters of small spheres," Surf. Sci. 156, 720-734.

Batson, P. E., 1993, "Simultaneous STEM imaging and electron-energy-loss spectroscopy with atomic-column sensitivity," Nature (London) 366, 727-728.

Batson, P. E., 2008, "Motion of gold atoms on carbon in the aberration-corrected STEM," Microsc. Microanal. 14, 89-97.

Batson, P. E., C. H. Chen, and J. Silcox, 1976, "Plasmon dispersion at large wave vectors in Al," Phys. Rev. Lett. 37, 937-940.

Batson, P. E., N. Dellby, and O. L. Krivanek, 2002, "Subangstrom resolution using aberration corrected electron optics," Nature (London) 418, 617-620.

Batson, P. E., and J. Silcox, 1983, "Experimental energy-loss function, $\operatorname{Im}[-1 / \epsilon(q, \omega)]$, for aluminum," Phys. Rev. B 27 , 5224-5239.

Bauer, E., 1994, "Low-energy-electron microscopy," Rep. Prog. Phys. 57, 895-938.

Berini, P., G. Mattiussi, N. Lahoud, and R. Charbonneau, 2007, "Wafer-bonded surface plasmon waveguides," Appl. Phys. Lett. 90, 061108.

Bertoni, G., and J. Verbeeck, 2008, “Accuracy and precision in model based EELS quantification," Ultramicroscopy $\mathbf{1 0 8}$ 782-790.

Bethe, H. A., 1930, "The theory of the passage of rapid neutron radiation through matter," Ann. Phys. 5, 325-400.

Betzig, E., P. L. Finn, and J. S. Weiner, 1992, "Combined shear force and near-field scanning optical microscopy," Appl. Phys. Lett. 60, 2484-2486.

Blackmore, V., G. Doucas, C. Perry, and M. F. Kimmitt, 2008, "First observation of coherent Smith-Purcell radiation in the highly relativistic regime," Nucl. Instrum. Methods Phys. Res. B 266, 3803-3810.

Blanco, L. A., and F. J. García de Abajo, 2004a, "Control of spontaneous emission by complex nanostructures," Opt. Lett. 29, 1494-1496.

Blanco, L. A., and F. J. García de Abajo, 2004b, "Spontaneous light emission in complex nanostructures," Phys. Rev. B 69, 205414.

Bloch, F., 1933, "Bremsvermögen von Atomen mit mehreren Elektronen," Z. Phys. 81, 363-376.

Boersch, H., J. Geiger, A. Imbusch, and N. Niedrig, 1966, "High resolution investigation of the energy losses of $30 \mathrm{keV}$ electrons in aluminum foils of various thicknesses," Phys. Lett. 22, 146-147.

Boersch, H., J. Geiger, and W. Stickel, 1966, "Interaction of $25-\mathrm{keV}$ electrons with lattice vibrations in LiF. Experimental evidence for surface modes of lattice vibration," Phys. Rev. Lett. 17, 379-381.

Boltasseva, A., and V. M. Shalaev, 2008, "Fabrication of optical negative-index metamaterials: Recent advances and outlook," Metamaterials 2, 1-17.

Bolton, J. P. R., and M. Chen, 1995, "Electron energy loss in multilayered slabs. I. Normal incidence," J. Phys.: Condens.
Matter 7, 3373-3387.

Borziak, P., I. Konovalov, Y. Kulyupin, and K. Pilipchak, 1976, "Cathodoluminescence from metal films," Thin Solid Films 35, L9-L12.

Bosman, M., V. J. Keast, M. Watanabe, A. I. Maaroof, and M. B. Cortie, 2007, "Mapping surface plasmons at the nanometre scale with an electron beam," Nanotechnology 18, 165505.

Bozhevolnyi, S. I., J. Erland, K. Leosson, P. M. W. Skovgaard, and J. M. Hvam, 2001, "Waveguiding in surface plasmon polariton band gap structures," Phys. Rev. Lett. 86, 3008-3011. Bozhevolnyi, S. I., V. S. Volkov, E. Devaux, J. Y. Laluet, and T. W. Ebbesen, 2006, "Channel plasmon subwavelength waveguide components including interferometers and ring resonators," Nature (London) 440, 508-511.

Bradley, J., et al., 2005, “An astronomical $2175 \AA$ feature in interplanetary dust particles," Science 307, 244-247.

Brambring, J., and H. Raether, 1965, "Plasma radiation from thin silver foils excited by light," Phys. Rev. Lett. 15, 882-883. Brink, H. A., M. M. G. Barfels, R. P. Burgner, and B. N. Edwards, 2003, "A sub-50 meV spectrometer and energy filter for use in combination with $200 \mathrm{kV}$ monochromated (S)TEMs," Ultramicroscopy 96, 367-384.

Brown, R. W., P. Wessel, and E. P. Trounson, 1960, "Plasmon reradiation from silver films," Phys. Rev. Lett. 5, 472-473.

Brownell, J. H., J. Walsh, and G. Doucas, 1998, "Spontaneous Smith-Purcell radiation described through induced surface currents," Phys. Rev. E 57, 1075-1080.

Browning, N. D., M. F. Chisholm, and S. J. Pennycook, 1993, "Atomic-resolution chemical analysis using a scanning transmission electron microscope," Nature (London) 366, 143-146. Brydson, R., 2001, Electron Energy Loss Spectroscopy (BIOS Scientific, Oxford).

Burda, C., X. Chen, R. Narayanan, and M. A. El-Sayed, 2005, "Chemistry and properties of nanocrystals of different shapes," Chem. Rev. (Washington, D.C.) 105, 1025-1102.

Cadete Santos Aires, F. J., A. Howie, and C. A. Walsh, 1993, "Valence loss electron spectroscopy of Ni-Al mixed oxides," J. Solid State Chem. 106, 48-54.

Cai, W., R. Sainidou, J. Xu, A. Polman, and F. J. García de Abajo, 2009, "Efficient generation of propagating plasmons by electron beams," Nano. Lett. 9, 1176-1181.

Calliari, L., S. Fanchenko, and M. Filippi, 2008, "Plasmon peak inhomogeneous broadening in reflection electron energy loss spectroscopy from carbon materials," Surf. Interface Anal. 40, 814-817.

Carusotto, I., M. Artoni, G. C. La Rocca, and F. Bassani, 2001, "Slow group velocity and Cherenkov radiation," Phys. Rev. Lett. 87, 064801.

Cazaux, J., 2006, "e-Induced secondary electron emission yield of insulators and charging effects," Nucl. Instrum. Methods Phys. Res. B 244, 307-322.

Chao, W., B. D. Harteneck, J. A. Liddle, E. H. Anderson, and D. T. Attwood, 2005, "Soft X-ray microscopy at a spatial resolution better than $15 \mathrm{~nm}$," Nature (London) 435, 1210-1213. Chaturvedi, P., K. H. Hsu, A. Kumar, K. H. Fung, J. C. Mabon, and N. X. Fang, 2009, "Imaging of plasmonic modes of silver nanoparticles using high-resoultion cathodoluminescence spectroscopy," ACS Nano 3, 2965-2974.

Chen, C. H., A. E. Meixner, and B. M. Kincaid, 1980, "Bulk plasmon dispersion in Si for $0<q<1.5 q_{F}$," Phys. Rev. Lett. 44, 951-954.

Chen, C. H., and J. Silcox, 1975a, "Detection of optical surface guided modes in thin graphite films by high-energy electron 
scattering," Phys. Rev. Lett. 35, 390-393.

Chen, C. H., and J. Silcox, 1975b, "Surface guided modes in an aluminum oxide thin film," Solid State Commun. 17, 273-275.

Chen, C. H., and J. Silcox, 1979, "Calculations of the electronenergy-loss probability in thin uniaxial crystals at oblique incidence," Phys. Rev. B 20, 3605-3614.

Chen, C. H., J. Silcox, A. F. Garito, A. J. Heeger, and A. G. MacDiarmid, 1976, "Plasmon dispersion and anisotropy in polymeric sulfur nitride, $(\mathrm{SN})_{x}$," Phys. Rev. Lett. 36, 525-528. Chen, C. H., J. Silcox, and R. Vincent, 1975, "Electron-energy losses in silicon: Bulk and surface plasmons and Cerenkov radiation," Phys. Rev. B 12, 64-71.

Chen, C. W., K. H. Chen, C. H. Shen, A. Ganguly, L. C. Chen, J. J. Wu, H. I. Wen, and W. F. Pong, 2006, "Anomalous blueshift in emission spectra of $\mathrm{ZnO}$ nanorods with sizes beyond quantum confinement regime," Appl. Phys. Lett. 88, 241905. Cherenkov, P. A., 1934, "The visible glow of pure liquids under the action of $\gamma$-rays," Dokl. Akad. Nauk SSSR 2, 451-454.

Chopra, N. G., R. J. Luyken, K. Cherrey, V. H. Crespi, M. L. Cohen, S. G. Louie, and A. Zettl, 1995, "Boron nitride nanotubes," Science 269, 966-967.

Chu, M. W., C. H. Chen, F. J. García de Abajo, J. P. Deng, and C. Y. Mou, 2008, "Surface exciton polaritons in individual $\mathrm{Au}$ nanoparticles in the far-ultraviolet spectral regime," Phys. Rev. B 77, 245402.

Chu, M. W., V. Myroshnychenko, C. H. Chen, J. P. Deng, C. Y. Mou, and F. J. García de Abajo, 2009, "Probing bright and dark surface-plasmon modes in individual and coupled noble metal nanoparticles using an electron beam," Nano Lett. 9, 399-404.

Čiljak, M., J. Ružička, A. S. Vodopianov, Y. I. Ivanshin, A. A. Tyapkin, I. A. Tyapkin, A. I. Zinchenko, and V. P. Zrelov, 2003, "Study of the particle radiation near the VavilovCherenkov radiation threshold," Nucl. Instrum. Methods Phys. Res. A 498, 126-134.

Cinchetti, M., A. Gloskovskii, S. A. Nepjiko, G. Schönhense, H. Rochholz, and M. Kreiter, 2005, "Photoemission electron microscopy as a tool for the investigation of optical near fields," Phys. Rev. Lett. 95, 047601.

Cohen, H., B. I. Lembrikov, M. A. Itskovsky, and T. Maniv, 2003, "Electromagnetic quantum-size effects in directional near-field EELS of nanocrystals," Nano Lett. 3, 203-206.

Cohen, H., T. Maniv, R. Tenne, Y. R. Hacohen, O. Stéphan, and C. Colliex, 1998, "Near-field electron energy loss spectroscopy of nanoparticles," Phys. Rev. Lett. 80, 782-785.

Cohen, H., T. Maniv, R. Tenne, Y. R. Hacohen, O. Stéphan, and C. Colliex, 1999, "Reply to 'Near-field electron energy loss spectroscopy of nanoparticles,'” Phys. Rev. Lett. 83, 659. Colas des Francs, G., C. Girard, J. C. Weeber, C. Chicane, T. David, A. Dereux, and D. Peyrade, 2001, "Optical analogy to electronic quantum corrals," Phys. Rev. Lett. 86, 4950-4953.

Cole, R. M., J. J. Baumberg, F. J. García de Abajo, S. Mahajan, M. Abdelsalam, and P. N. Bartlett, 2007, "Understanding plasmons in nanoscale voids," Nano Lett. 7, 2094-2100.

Couillard, M., M. Kociak, O. Stéphan, G. A. Botton, and C. Colliex, 2007, "Multiple-interface coupling effects in local electron-energy-loss measurements of band gap energies," Phys. Rev. B 76, 165131.

Couillard, M., A. Yurtsever, and D. A. Muller, 2008, "Competition between bulk and interface plasmonic modes in valence electron energy-loss spectroscopy of ultrathin $\mathrm{SiO}_{2}$ gate stacks," Phys. Rev. B 77, 085318.

Cowley, J. M., 1982a, "Electron losses of fast electrons at crys- tal surfaces," Phys. Rev. B 25, 1401-1404.

Cowley, J. M., 1982b, "Surface energies and surface structure of small crystals studied by use of a STEM instrument," Surf. Sci. 114, 587-606.

Coyle, S., M. C. Netti, J. J. Baumberg, M. A. Ghanem, P. R. Birkin, P. N. Bartlett, and D. M. Whittaker, 2001, "Confined plasmons in metallic nanocavities," Phys. Rev. Lett. 87, 176801.

Cram, L. S., and E. T. Arakawa, 1967, "Bremsstrahlung and transition radiation from $\mathrm{Ag}$ foils bombarded by non-normal incidence electrons," Phys. Rev. 153, 455-459.

Creuzburg, M., 1966, "Entstehung von Alkalimetallen bei der Elektronenbestrahlung von Alkalihalogeniden(Nachgewiesen mit Plasmaverlusten und Elektroneinterferenzen)," Z. Phys. 194, 211-218.

Crewe, A. V., M. Isaacson, and D. Johnson, 1971, "Electron energy loss spectra of the nucleic acid bases," Nature (London) 231, 262-263.

Crowell, J., and R. H. Ritchie, 1968, "Radiative decay of Coulomb-stimulated plasmons in spheres," Phys. Rev. 172, 436-440.

D'Aguanno, G., N. Mattiucci, M. Centini, M. Scalora, and M. J. Bloemer, 2004, "Electromagnetic density of modes for a finite-size three-dimensional structure," Phys. Rev. E 69, 057601.

Daniels, H. R., R. Brydson, A. Brown, and B. Rand, 2003, "Quantitative valence plasmon mapping in the TEM: Viewing physical properties at the nanoscale," Ultramicroscopy 96, 547-558.

Davis, L. C., 1976, "Electrostatic edge modes of a dielectric wedge," Phys. Rev. B 14, 5523-5525.

de Broglie, L., 1925, "Recherches sur la théorie des quanta," Ann. Phys. (Paris) 3, 22-128.

Degiron, A., H. J. Lezec, N. Yamamoto, and T. W. Ebbesen, 2004, "Optical transmission properties of a single subwavelength aperture in a real metal," Opt. Commun. 239, 61-66.

Demkov, Y. N., and J. D. Meyer, 2004, "A sub-atomic microscope, superfocusing in channeling and close encounter atomic and nuclear reactions," Eur. Phys. J. B 42, 361-365.

Denisyuk, A. I., F. Jonsson, K. F. MacDonald, N. I. Zheludev, and F. J. García de Abajo, 2008, "Luminescence readout of nanoparticle phase state," Appl. Phys. Lett. 92, 093112.

Diaconescu, B., K. Pohl, L. Vattuone, L. Savio, P. Hofmann, V. M. Silkin, J. M. Pitarke, E. V. Chulkov., P. M. Echenique, D. Farías, and M. Rocca, 2007, "Low-energy acoustic plasmons at metal surfaces," Nature (London) 448, 57-59.

Dobrzynski, L., and A. A. Maradudin, 1972, "Electrostatic edge modes in a dielectric wedge," Phys. Rev. B 6, 3810-3815. Dorneich, A. D., R. H. French, H. Müllejans, S. Loughin, and M. Rühle, 1998, "Quantitative analysis of valence electron energy-loss spectra of aluminium nitride," J. Microsc. 191, 286-296.

Doucas, G., J. H. Mulvey, M. Omori, J. Walsh, and M. F. Kimmitt, 1992, "First observation of Smith-Purcell radiation from relativistic electrons," Phys. Rev. Lett. 69, 1761-1764.

Drachsel, W., M. Adelt, N. Nilius, and H. J. Freund, 2002, "Cathodoluminescence of small silver particles on $\mathrm{Al}_{2} \mathrm{O}_{3} / \mathrm{NiAl}$ (110)," J. Electron Spectrosc. Relat. Phenom. 122, 239-249.

Draine, B. T., and P. J. Flatau, 1994, "Discrete-dipole approximation for scattering calculations," J. Opt. Soc. Am. A 11, 1491-1499.

Drezet, A., A. Hohenau, J. R. Krenn, M. Brun, and S. Huant, 
2007, "Surface plasmon mediated near-field imaging and optical addressing in nanoscience," Micron 38, 427-437.

Drucker, J., and M. R. Scheinfein, 1993, "Delocalized secondary-electron generation studied by momentumresolved coincidence-electron spectroscopy," Phys. Rev. B 47, 15973-15975.

Drucker, J., M. R. Scheinfein, J. Liu, and J. K. Weiss, 1993, "Electron coincidence spectroscopy studies of secondary and Auger electron generation mechanisms," J. Appl. Phys. 74, 7329-7339.

Dulkeith, E., T. Niedereichholz, T. A. Klar, J. Feldmann, G. von Plessen, D. I. Gittins, K. S. Mayya, and F. Caruso, 2004, "Plasmon emission in photoexcited gold nanoparticles," Phys. Rev. B 70, 205424.

Dwyer, C., R. Erni, and J. Etheridge, 2008, "Method to measure spatial coherence of subangstrom electron beams," Appl. Phys. Lett. 93, 021115.

Echenique, P. M., J. Bausells, and A. Rivacoba, 1987, "Energyloss probability in electron microscopy," Phys. Rev. B 35, 1521-1524.

Echenique, P. M., F. Flores, and R. H. Ritchie, 1990, "Dynamic screening of ions in condensed matter," Solid State Phys. 43, 229-308.

Echenique, P. M., A. Howie, and R. H. Ritchie, 1999, "Comment on 'Near-field electron energy loss spectroscopy of nanoparticles,'” Phys. Rev. Lett. 83, 658.

Echenique, P. M., and J. B. Pendry, 1975, “Absorption profile at surfaces," J. Phys. C 8, 2936-2942.

Edighoffer, J. A., W. D. Kimura, R. H. Pantell, M. A. Piestrup, and D. Y. Wang, 1981, "Observation of inverse Cherenkov interaction between free electrons and laser light," Phys. Rev. A 23, 1848-1854.

Egerton, R. F., 1996, Electron Energy-Loss Spectroscopy in the Electron Microscope (Plenum, New York).

Egerton, R. F., 2003, "New techniques in electron energy-loss spectroscopy and energy-filtered imaging," Micron 34, 127 139.

Egerton, R. F., 2007, "Limits to the spatial, energy and momentum resolution of electron energy-loss spectroscopy," Ultramicroscopy 107, 575-586.

Egerton, R. F., 2009, "Electron energy-loss spectroscopy in the TEM,” Rep. Prog. Phys. 72, 016502.

Eggeman, A. S., P. J. Dobson, and A. K. Petford-Long, 2007, "Optical spectroscopy and energy-filtered transmission electron microscopy of surface plasmons in core-shell nanoparticles," J. Appl. Phys. 101, 024307.

Eguiluz, A., and A. A. Maradudin, 1976, "Electrostatic edge modes along a parabolic wedge," Phys. Rev. B 14, 5526-5528.

Erni, R., and N. D. Browning, 2005, "Valence electron energyloss spectroscopy in monochromated scanning transmission electron microscopy," Ultramicroscopy 104, 176-192.

Erni, R., and N. D. Browning, 2007, "Quantification of the size-dependent energy gap of individual CdSe quantum dots by valence electron energy-loss spectroscopy," Ultramicroscopy $107,267-273$.

Erni, R., and N. D. Browning, 2008, "The impact of surface and retardation losses on valence electron energy-loss spectroscopy," Ultramicroscopy 108, 84-99.

Erni, R., S. Lazar, and N. D. Browning, 2008, "Prospects for analyzing the electronic properties in nanoscale systems by VEELS," Ultramicroscopy 108, 270-276.

Fehlhaber, R. P., and L. A. Bursill, 1999, "Plasmon excitations in small diamond spheres by fast penetrating electrons,"
Phys. Rev. B 60, 14147-14153.

Fehlhaber, R. P., and L. A. Bursill, 2000, "Plasmon excitations in coated nanocrystalline diamond spheres," Phys. Rev. B 62, 17094-17102.

Fermi, E., 1940, "The ionization loss of energy in gases and in condensed materials," Phys. Rev. 57, 485-493.

Ferrell, R. A., 1958, "Predicted radiation of plasma oscillations in metal films," Phys. Rev. 111, 1214-1222.

Ferrell, T. L., and P. M. Echenique, 1985, "Generation of surface excitations on dielectric spheres by an external electron beam," Phys. Rev. Lett. 55, 1526-1529.

Ferrell, T. L., R. J. Warmack, V. E. Anderson, and P. M. Echenique, 1987, "Analytical calculation of stopping power for isolated small spheres," Phys. Rev. B 35, 7365-7371.

Ford, G. W., and W. H. Weber, 1984, "Electromagnetic interactions of molecules with metal surfaces," Phys. Rep. 113, 195-287.

Forstmann, F., A. Gras-Martí, T. L. Ferrell, R. J. Warmack, and K. C. Mamola, 1991, "Energy loss of low-energy electrons to nonabrupt metal surfaces," Phys. Rev. B 44, 48844891.

Frank, I. M., and I. Tamm, 1937, "Coherent visible radiation of fast electrons passing through matter," Dokl. Akad. Nauk SSSR 14, 109-114.

French, R. H., H. Müllejans, and D. J. Jones, 1998, "Optical properties of aluminum oxide: Determined from vacuum ultraviolet and electron energy-loss spectroscopies," J. Am. Ceram. Soc. 81, 2459-2557.

Fu, L., P. B. Macedo, and L. Resca, 1993, "Analytic approach to the intarfacial polarization of heterogeneous systems," Phys. Rev. B 47, 13818-13829.

Fu, L., and L. Resca, 1993, "Electrical response of heterogeneous systems of clustered inclusions," Phys. Rev. B 47, 16194-16204.

Fuchs, R., 1975, "Theory of the optical properties of ionic crystal cubes," Phys. Rev. B 11, 1732-1740.

Fuchs, R., R. G. Barrera, and J. L. Carrillo, 1996, "Spectral representations of the electron energy loss in composite media," Phys. Rev. B 54, 12824-12834.

Fuchs, R., and K. L. Kliewer, 1968, "Optical modes of vibration in an ionic crystal scphere," J. Opt. Soc. Am. 58, 319-330. Fujimoto, F., and K. Komaki, 1968, "Plasma oscillations excited by a fast electron in a metallic particle," J. Phys. Soc. Jpn. 25, 1679-1687.

Fujimoto, F., K. Komaki, and K. Ishida, 1967, "Surface plasma oscillation in aluminum fine particles," J. Phys. Soc. Jpn. 23, 1186.

Fussell, D. P., R. C. McPhedran, and C. Martijn de Sterke, 2004, "Three-dimensional Green's tensor, local density of states, and spontaneous emission in finite two-dimensional photonic crystals composed of cylinders," Phys. Rev. E 70, 066608.

Fussell, D. P., R. C. McPhedran, and C. Martijn de Sterke, 2005, "Decay rate and level shift in a circular dielectric waveguide," Phys. Rev. A 71, 013815.

Galloway, S. A., P. Miller, P. Thomas, and R. Harmon, 2003, "Advances in cathodoluminescence characterisation of compound semiconductors with spectrum imaging," Phys. Status Solidi C 0, 1028-1032.

Gans, R., 1912, "The shape of ultra microscopic gold particles," Ann. Phys. 37, 881-900.

García de Abajo, F. J., 1999a, "Interaction of radiation and fast electrons with clusters of dielectrics: A multiple scattering 
approach," Phys. Rev. Lett. 82, 2776-2779.

García de Abajo, F. J., 1999b, "Relativistic description of valence energy losses in the interaction of fast electrons with clusters of dielectrics: Multiple-scattering approach," Phys. Rev. B 60, 6103-6112.

García de Abajo, F. J., 1999c, "Relativistic energy loss and induced photon emission in the interaction of a dielectric sphere with an external electron beam," Phys. Rev. B 59, 3095-3107.

García de Abajo, F. J., 2004, "Momentum transfer to small particles by passing electron beams,” Phys. Rev. B 70, 115422. García de Abajo, F. J., 2007, "Light scattering by particle and hole arrays," Rev. Mod. Phys. 79, 1267-1290.

García de Abajo, F. J., 2008, "Nonlocal effects in the plasmons of strongly interacting nanoparticles, dimers, and waveguides,” J. Phys. Chem. C 112, 17983-17987.

García de Abajo, F. J., 2009, "Optical emission from the interaction of fast electrons with metallic films containing a circular aperture: A study of radiative decoherence of fast electrons," Phys. Rev. Lett. 102, 237401.

García de Abajo, F. J., and J. Aizpurua, 1997, "Numerical simulation of electron energy loss near inhomogeneous dielectrics," Phys. Rev. B 56, 15873-15884.

García de Abajo, F. J., and L. A. Blanco, 2003, "Electron energy loss and induced photon emission in photonic crystals," Phys. Rev. B 67, 125108.

García de Abajo, F. J., and P. M. Echenique, 1992, "Wake potential in the vicinity of a surface," Phys. Rev. B 46, 26632675.

García de Abajo, F. J., and A. Howie, 1998, "Relativistic electron energy loss and electron-induced photon emission in inhomogeneous dielectrics," Phys. Rev. Lett. 80, 5180-5183.

García de Abajo, F. J., and A. Howie, 2002, "Retarded field calculation of electron energy loss in inhomogeneous dielectrics," Phys. Rev. B 65, 115418.

García de Abajo, F. J., and M. Kociak, 2008a, "Electron energy-gain spectroscopy," New J. Phys. 10, 073035.

García de Abajo, F. J., and M. Kociak, 2008b, "Probing the photonic local density of states with electron energy loss spectroscopy,” Phys. Rev. Lett. 100, 106804.

García de Abajo, F. J., A. G. Pattantyus-Abraham, N. Zabala, A. Rivacoba, M. O. Wolf, and P. M. Echenique, 2003, "Cherenkov effect as a probe of photonic nanostructures," Phys. Rev. Lett. 91, 143902.

García de Abajo, F. J., A. Rivacoba, N. Zabala, and N. Yamamoto, 2004, "Boundary effects in Cherenkov radiation," Phys. Rev. B 69, 155420.

García-Molina, R., A. Gras-Martí, A. Howie, and R. H. Ritchie, 1985, "Retardation effects in the interaction of charged particle beams with bounded condensed media," J. Phys. C 18, 5335-5345.

García-Molina, R., A. Gras-Martí, and R. H. Ritchie, 1985, "Excitation of edge modes in the interaction of electron beams with dielectric wedges," Phys. Rev. B 31, 121-126.

Gemmell, D. S., J. Remillieux, J. C. Poizat, M. J. Gaillard, R. E. Holland, and Z. Vager, 1975, "Evidence for an alignment effect in the motion of swift ion clusters through solids," Phys. Rev. Lett. 34, 1420-1424.

Genet, C., and T. W. Ebbesen, 2007, "Light in tiny holes," Nature (London) 445, 39-46.

Gensterblum, G., J. J. Pireaux, P. A. Thiry, R. Caudano, J. P. Vigneron, P. Lambin, A. A. Lucas, and W. Krätschmer, 1991, "High-resolution electron-energy-loss spectroscopy of thin films of $\mathrm{C}_{60}$ on $\mathrm{Si}(100)$," Phys. Rev. Lett. 67, 2171-2174.

Ginzburg, V. L., 1996, "Radiation by uniformly moving sources (Vavilov-Cherenkov effect, transition radiation, and other phenomena)," Phys. Usp. 39, 973-982.

Ginzburg, V. L., and I. M. Frank, 1946, "Radiation of a uniformly moving electron due to its transition from one medium to another," Zh. Eksp. Teor. Fiz. 16, 1528 [J. Phys. (USSR) 9, 353-362 (1945)].

Gobin, A. M., M. H. Lee, N. J. Halas, W. D James, R. A. Drezek, and J. L. West, 2007, "Near-infrared resonant nanoshells for combined optical imaging and photothermal cancer therapy," Nano Lett. 7, 1929-1934.

Goldobin, E., A. Wallraff, N. Thyssen, and A. V. Ustinov, 1998, "Cherenkov radiation in coupled long Josephson junctions," Phys. Rev. B 57, 130-133.

Goldsmith, P., and J. V. Jelley, 1959, "Optical transition radiation from protons entering metal surfaces," Philos. Mag. 4, 836-844.

Gómez-Medina, R., N. Yamamoto, M. Nakano, and F. J. García de Abajo, 2008, "Mapping plasmons in nanoantennas via cathodoluminescence," New J. Phys. 10, 105009.

Gordon, J. P., and A. Ashkin, 1980, "Motion of atoms in a radiation trap," Phys. Rev. A 21, 1606-1617.

Grier, D. G., 2003, "A revolution in optical manipulation," Nature (London) 424, 810-816.

Grinolds, M. S., V. A. Lobastov, J. Weissenrieder, and A. H. Zewail, 2006, "Four-dimensional ultrafast electron microscopy of phase transitions," Proc. Natl. Acad. Sci. U.S.A. 103, 18427-18431.

Grundmann, M., J. Christen, N. N. Ledentsov, J. Böhrer, D. Bimberg, S. S. Ruvimov, P. Werner, U. Richter, U. Gösele, J. Heydenreich, V. M. Ustinov, A. Y. Egorov, E. Zhukals, P. S. Kopevs, and Z. I. Alferov, 1995, "Ultranarrow luminescence lines from single quantum dots," Phys. Rev. Lett. 74, 40434046.

Grzelczak, M., J. Pérez-Juste, F. J. García de Abajo, and L. M. Liz-Marzán, 2007, "Optical properties of platinum-coated gold nanorods," J. Phys. Chem. C 111, 6183-6188.

Gu, L., V. Srot, W. Sigle, C. Koch, P. van Aken, F. Scholz, S. B. Thapa, C. Kirchner, M. Jetter, and M. Rühle, 2007, "Bandgap measurements of direct and indirect semiconductors using monochromated electrons," Phys. Rev. B 75, 195214.

Guan, L., K. Suenaga, Z. Shi, Z. Gu, and S. Iijima, 2005, "Direct imaging of the alkali metal site in K-doped fullerene peapods," Phys. Rev. Lett. 94, 045502.

Guck, J., R. Ananthakrishnan, T. J. Moon, C. C. Cunningham, and J. Käs, 2000, "Optical deformability of soft biological dielectrics,” Phys. Rev. Lett. 84, 5451-5454.

Haeberlé, O., P. Rullhusen, J. M. Salomé, and N. Maene, 1994, "Calculations of Smith-Purcell radiation generated by electrons of 1-100 MeV," Phys. Rev. E 49, 3340-3352.

Hanrath, T., and B. A. Korgel, 2004, “A comprehensive study of electron energy losses in Ge nanowires," Nano Lett. 4, 1455-1461.

Hartschuh, A., 2008, "Tip-enhanced near-field optical microscopy,” Angew. Chem., Int. Ed. 47, 8178-8191.

Hattendorff, H. D., 1977, "Transition radiation from gold and silver films bombarded with $80 \mathrm{keV}$ electrons," Phys. Status Solidi A 42, 489-494.

Haydock, R., 1980, "The recursive solution of the Schrödinger equation," Solid State Phys. 35, 215-294.

Heitmann, D., 1977, "Radiative decay of surface plasmons excited by fast electrons on periodically modulated silver plas- 
mons," J. Phys. C 10, 397-405.

Henrard, L., and P. Lambin, 1996, "Calculation of the energy loss for an electron passing near giant fullerenes," J. Phys. B 29, 5127-5141.

Henrard, L., F. Malengreau, P. Rudolf, K. Hevesi, R. Caudano, P. Lambin, and T. Cabioc'h, 1999, "Electron-energy-loss spectroscopy of plasmon excitations in concentric-shell fullerenes," Phys. Rev. B 59, 5832-5836.

Herring, R. A., 2005, "Energy-filtered electron-diffracted beam holography," Ultramicroscopy 104, 261-270.

Herring, R. A., 2008, "Planar diffracted-beam interferometry/ holography," Ultramicroscopy 108, 688-697.

Hillier, J., and R. F. Baker, 1944, "Microanalysis by means of electrons," J. Appl. Phys. 15, 663-675.

Hofmann, C. E., E. J. R. Vesseur, L. A. Sweatlock, H. J. Lezec, F. J. García de Abajo, A. Polman, and H. A. Atwater, 2007, "Plasmon modes of annular nanoresonators imaged by spectrally resolved cathodoluminescence," Nano Lett. 7, 36123617.

Hohenberg, P., and W. Kohn, 1964, "Inhomogeneous electron gas,” Phys. Rev. 136, B864-B871.

Hohenester, U., H. Ditlbacher, and J. R. Krenn, 2009, "Electron-energy-loss spectra of plasmonic nanoparticles," Phys. Rev. Lett. 103, 106801.

Hohenester, U., and J. Krenn, 2005, "Surface plasmon resonances of single and coupled metallic nanoparticles: A boundary integral method approach," Phys. Rev. B 72, 195429.

Holt, D. B., and B. G. Yacobi, 1990, Cathodoluminescence Microscopy of Inorganic Solids (Plenum, New York).

Horiuchi, N., T. Ochiai, J. Inoue, Y. Segawa, Y. Shibata, K. Ishi, Y. Kondo, M. Kanbe, H. Miyazaki, F. Hinode, S. Yamaguti, and K. Ohtaka, 2006, "Exotic radiation from a photonic crystal excited by an ultrarelativistic electron beam," Phys. Rev. E 74, 056601.

Hörmandinger, G., 1994, "Imaging of the $\mathrm{Cu}(111)$ surface state in scanning tunneling microscopy," Phys. Rev. B 49, 1389713905.

Hövel, H., S. Fritz, A. Hilger, U. Kreibig, and M. Vollmer, 1993, "Width of cluster plasmon resonances: Bulk dielectric functions and chemical interface damping," Phys. Rev. B 48, 18178-18188.

Howie, A., 1999, "Electrons and photons: exploiting the connection," Inst. Phys. Conf. Ser. 161, 311-314.

Howie, A., 2003, "Valence excitations in electron microscopy: Resolved and unresolved issues," Micron 34, 121-125.

Howie, A., 2009, "Photon-assisted electron energy loss spectroscopy and ultrafast imaging," Microsc. Microanal. 15, 314 322.

Howie, A., and R. H. Milne, 1985, "Excitations at interfaces and small particles," Ultramicroscopy 18, 427-433.

Howie, A., and C. Walsh, 1991, "Interpretation of valence loss spectra from composite media," Microsc. Microanal. Microstruct. 2, 171-181.

Hyun, J. K., M. Couillard, P. Rajendran, C. M. Liddell, and D. A. Muller, 2008, "Measuring far-ultraviolet whispering gallery modes with high energy electrons," Appl. Phys. Lett. 93, 243106.

Iijima, S., 1991, "Helical microtubules of graphitic carbon," Nature (London) 354, 56-58.

Illman, B. L., V. E. Anderson, R. J. Warmack, and T. L. Ferrell, 1988, "Spectrum of surface-mode contributions to the differential energy-loss probability for electrons passing by a spher- oid," Phys. Rev. B 38, 3045-3049.

Imbusch, A., and H. Niedrig, 1970, "Temperature effect on energy loss spectrum of fast electrons in aluminium and lead foils between $3 \mathrm{~K}$ and $295 \mathrm{~K}$," Phys. Lett. 32A, 375-376.

Intaraprasonk, V., H. L. Xin, and D. A. Muller, 2008, "Analytic derivation of optimal imaging conditions for incoherent imaging in aberration-corrected electron microscopes," Ultramicroscopy 108, 1454-1466.

Ireland, M. J., A. Kraus, F. Martinache, J. P. Lloyd, and P. G. Tuthill, 2008, "Dynamical mass of GJ 802B: A brown dwarf in a triple system," Astrophys. J. 678, 463-471.

Itskovsky, M. A., H. Cohen, and T. Maniv, 2008, "Radiative interaction of a focused relativistic electron beam in energyloss spectroscopy of nanoscopic platelets," Phys. Rev. B 78, 045419.

Jackson, J. D., 1999, Classical Electrodynamics (Wiley, New York).

Jiang, N., D. Su, J. C. H. Spence, S. Zhou, and J. Qiu, 2008, "Electron energy loss spectroscopy of $\mathrm{Na}$ in $\mathrm{Na}, \mathrm{Na}_{2} \mathrm{O}$, and silicate glasses," J. Mater. Res. 23, 2467-2471.

Johnson, P. B., and R. W. Christy, 1972, "Optical constants of the noble metals," Phys. Rev. B 6, 4370-4379.

Jones, R. C., 1945, “A generalization of the dielectric ellipsoid problem," Phys. Rev. 68, 93-96.

Jouffrey, B., P. Schattschneider, and C. Hébert, 2004, "The magic angle: A solved mystery," Ultramicroscopy 102, 61-66. Joulain, K., R. Carminati, J. P. Mulet, and J. J. Greffet, 2003, "Definition and measurement of the local density of electromagnetic states close to an interface," Phys. Rev. B 68, 245405.

Julley, J. V., 1958, Cherenkov Radiation and its Application (Pergamon, New York).

Karali, T., N. Can, L. Valberg, A. L. Stepanov, P. D. Townsend, C. Buchal, R. A. Ganeev, A. I. Ryasnyansky, H. G. Belik, M. L. Jessett, and C. Ong, 2005, "Optical properties and luminescence of metallic nanoclusters in $\mathrm{ZnO}: \mathrm{Cu}$," Physica B 363, 88-95.

Karataev, P., S. Araki, R. Hamatsu, H. Hayano, T. Muto, G. Naumenko, A. Potylitsyn, N. Terunuma, and J. Urakawa, 2004, "Beam-size measurement with optical diffraction radiation at KEK accelerator test facility," Phys. Rev. Lett. 93, 244802.

Keast, V. J., and M. Bosman, 2008, “Applications and theoretical simulation of low-loss electron energy-loss spectra," Mater. Sci. Technol. 24, 651-659.

Keller, J. W., and M. A. Coplan, 1992, "Electron energy loss spectroscopy of $\mathrm{C}_{60}$," Chem. Phys. Lett. 193, 89-92.

Kelly, K. L., E. Coronado, L. L. Zhao, and G. C. Schatz, 2003, "The optical properties of metal nanoparticles: The influence of size, shape, and dielectric environment," J. Phys. Chem. B 107, 668-677.

Kim, J., and K. B. Song, 2007, "Recent progress of nanotechnology with NSOM," Micron 38, 409-426.

Kimura, W. D., G. H. Kim, R. D. Romea, L. C. Steinhauer, I. V. Pogorelsky, K. P. Kusche, R. C. Fernow, X. Wang, and Y. Liu, 1995, "Laser acceleration of relativistic electrons using the inverse Cherenkov effect," Phys. Rev. Lett. 74, 546-549.

Kitson, S. C., W. L. Barnes, and J. R. Sambles, 1996, "Full photonic band gap for surface modes in the visible," Phys. Rev. Lett. 77, 2670-2673.

Kittle, C., 1987, Quantum Theory of Solids (Wiley, New York). Kliewer, K. L., and R. Fuchs, 1967, "Collective electronic motion in a metallic slab," Phys. Rev. 153, 498-512. 
Kociak, M., L. Henrard, O. Stéphan, K. Suenaga, and C. Colliex, 2000, "Plasmons in layered nanospheres and nanotubes investigated by spatially resolved electron energy-loss spectroscopy," Phys. Rev. B 61, 13936-13944.

Kociak, M., O. Stéphan, L. Henrard, V. Charbois, A. Rothschild, R. Tenne, and C. Colliex, 2001, "Experimental evidence of surface-plasmon coupling in anisotropic hollow nanoparticles,” Phys. Rev. Lett. 87, 075501.

Koh, A. L., K. Bao, I. Khan, W. E. Smith, G. Kothleitner, P. Nordlander, S. A. Maier, and D. W. McComb, 2009, "Electron-energy-loss spectroscopy (EELS) of surface plasmons in single silver nanoparticles and dimers: Influence of beam damage and mapping of dark modes," ACS Nano 3, 3015-3022.

Kohl, H., 1983, "Image formation by inelastically scattered electrons: Image of a surface plasmon," Ultramicroscopy 11, 53-65.

Koizumi, S., K. Watanabe, M. Hasegawa, and H. Kanda, 2001, "Ultraviolet emission from a diamond pn junction," Science 292, 1899-1901.

Korda, P. T., M. B. Taylor, and D. G. Grier, 2002, "Kinetically locked-in colloidal transport in an array of optical tweezers," Phys. Rev. Lett. 89, 128301.

Krane, K. J., and H. Raether, 1976, "Measurement of surface plasmon dispersion in aluminum and indium," Phys. Rev. Lett. 37, 1355-1357.

Kreibig, U., and M. Vollmer, 1995, Optical Properties of Metal Clusters (Springer-Verlag, Berlin).

Kremers, C., D. N. Chigrin, and J. Kroha, 2009, "Theory of Cherenkov radiation in periodic dielectric media: Emission spectrum," Phys. Rev. A 79, 013829.

Krivanek, O. L., N. Dellby, and A. R. Lupini, 1999, "Towards sub- $\AA$ electron beams," Ultramicroscopy 78, 1-11.

Kröger, E., 1968, "Berechnung der Energieverluste schneller Elektronen in dünnen Schichten mit Retardierung," Z. Phys. 216, 115-135.

Kroto, H. W., J. R. Heath, S. C. O'Brien, R. F. Curl, and R. E. Smalley, 1985, " $\mathrm{C}_{60}$ : Buckminsterfullerene," Nature (London) 318, 162-163.

Kubo, A., K. Onda, H. Petek, Z. Sun, Y. S. Jung, and H. K. Kim, 2005, "Femtosecond imaging of surface plasmon dynamics in a nanostructured silver film," Nano Lett. 5, 1123 1127.

Kubo, A., N. Pontius, and H. Petek, 2007, "Femtosecond microscopy of surface plasmon polariton wave packet evolution at the silver/vacuum interface," Nano Lett. 7, 470-475.

Kumar, P. S., I. Pastoriza-Santos, B. Rodríguez-González, F. J. García de Abajo, and L. M. Liz-Marzán, 2008, "High-yield synthesis and optical response of gold nanostars," Nanotechnology 19, 015606.

Kuttge, M., W. Cai, F. J. García de Abajo, and A. Polman, 2009, "Dispersion of metal-insulator-metal plasmon polaritons probed by cathodoluminescence imaging spectroscopy," Phys. Rev. B 80, 033409.

Kuttge, M., E. J. R. Vesseur, A. F. Koenderink, H. J. Lezec, H. A. Atwater, F. J. García de Abajo, and A. Polman, 2009, "Local density of states, spectrum, and far-field interference of surface plasmon polaritons probed by cathodoluminescence," Phys. Rev. B 79, 113405.

Kuttge, M., E. J. R. Vesseur, and A. Polman, 2009, "FabryPerot resonators for surface plasmon polaritons probed by cathodoluninescence," Appl. Phys. Lett. 94, 183104.

Kuttge, M., E. J. R. Vesseur, J. Verhoeven, H. J. Lezec, H. A.
Atwater, and A. Polman, 2008, "Loss mechanisms of surface plasmon polaritons on gold probed by cathodoluminescence imaging spectroscopy," Appl. Phys. Lett. 93, 113110.

Kuzuo, R., M. Terauchi, and M. Tanaka, 1992, "Electron energy-loss spectra of carbon nanotubes," Jpn. J. Appl. Phys., Part 2 31, L1484-L1487.

Lalor, E., and E. Wolf, 1971, "New model for the interaction between a moving charged particle and a dielectric, and the Cherenkov effect," Phys. Rev. Lett. 26, 1274-1277.

Landau, L. D., E. M. Lifshitz, and L. P. Pitaevskii, 1984, Electrodynamics of Continuous Media (Pergamon, Oxford).

Lang, W., 1948, "Geschwindigkeitsuerluste mittleschneller elektronen keine durchgang durch dinne metallfolien," Optik (Jena) 3, 233-246.

Larkin, I. A., and M. I. Stockman, 2005, "Imperfect perfect lens," Nano Lett. 5, 339-343.

Larkin, I. A., M. I. Stockman, M. Achermann, and V. I. Klimov, 2004, "Dipolar emitters at nanoscale proximity of metal surfaces: Giant enhancement of relaxation in microscopic theory," Phys. Rev. B 69, 121403(R).

Lastdrager, B., A. Tip, and J. Verhoeven, 2000, "Theory of Cherenkov and transition radiation from layered structures," Phys. Rev. E 61, 5767-5778.

Lazar, S., G. A. Botton, M. Y. Wu, F. D. Tichelaar, and H. W. Zandbergen, 2003, "Materials science applications of HREELS in near edge structure analysis and low-energy loss spectroscopy," Ultramicroscopy 96, 535-546.

Lazar, S., G. A. Botton, and H. W. Zandbergen, 2006, "Enhancement of resolution in core-loss and low-loss spectroscopy in a monochromated microscope," Ultramicroscopy 106, 1091-1103.

Lecante, J., Y. Ballu, and D. M. Newns, 1977, "Electronsurface-plasmon scattering using a parabolic nontouching trajectory," Phys. Rev. Lett. 38, 36-40.

Leithäuser, G. E., 1904, "Über den Geschwindigkeitsverlust, welchen die Kathodenstrahlen beim Durchgang durch dünne Metallschichten erleiden, und über die Ausmessung magnetischer Spektren," Ann. Phys. 15, 283-306.

Li, D., K. Imasaki, X. Gao, Z. Yang, and G. S. Park, 2007, "Reduce the start current of Smith-Purcell backward wave oscillator by sidewall grating," Appl. Phys. Lett. 91, 221506. Li, D., K. Imasaki, Z. Yang, and G. S. Park, 2006, “Threedimensional simulation of super-radiant Smith-Purcell radiation,” Appl. Phys. Lett. 88, 201501.

Li, D., K. Imasaki, Z. Yang, G. S. Park, S. Miyamoto, S. Amano, and T. Mochizuki, 2007, "Effect of grating surface loss on the Smith-Purcell free-electron laser," Nucl. Instrum. Methods Phys. Res. A 572, 948-952.

Lichte, H., and B. Freitag, 2000, "Inelastic electron holography," Ultramicroscopy 81, 177-186.

Lindhard, J., 1954, "On the properties of a gas of charged particles,” K. Dan. Vidensk. Selsk. Mat. Fys. Medd. 28, 1-57.

Little, J. W., T. A. Callcott, T. L. Ferrell, and E. T. Arakawa, 1984, "Surface-plasmon radiation from ellipsoidal silver spheroids," Phys. Rev. B 29, 1606-1615.

Liu, W., Z. Yang, Z. Liang, D. Li, K. Imasaki, Z. Shi, F. Lan, and G. S. Park, 2007, "Enhancement of terahertz SmithPurcell radiation by two electron beams," Nucl. Instrum. Methods Phys. Res. A 580, 1552-1558.

Lobastov, V. A., R. Srinivasan, and A. H. Zewail, 2005, "Fourdimensional ultrafast electron microscopy," Proc. Natl. Acad. Sci. U.S.A. 102, 7069-7073. 
Loudon, R., 2000, The Quantum Theory of Light (Oxford University Press, Oxford).

Lu, J. Q., and A. A. Maradudin, 1990, "Channel plasmons," Phys. Rev. B 42, 11159-11165.

Lucas, A. A., L. Henrard, and P. Lambin, 1994, "Computation of the ultraviolet absorption and electron inelastic scattering cross section of multishell fullerenes," Phys. Rev. B 49, 28882896.

Lucas, A. A., and E. Kartheuser, 1970, "Energy-loss spectrum of fast electrons in a dielectric slab. I. Nonretarded losses and Cherenkov bulk loss," Phys. Rev. B 1, 3588-3598.

Lucas, A. A., and M. Šunjić, 1971, "Fast-electron spectroscopy of surface excitations," Phys. Rev. Lett. 26, 229-232.

Luo, C., M. Ibanescu, S. G. Johnson, and J. D. Joannopoulos, 2003, "Cherenkov radiation in photonic crystals," Science 299, 368-371.

Ma, D. D. D., S. T. Lee, P. Mueller, and S. F. Alvarado, 2006, "Scanning tunneling microscope excited cathodoluminescence from ZnS nanowires," Nano Lett. 6, 926-929.

Macaulay, J. M., R. M. Allen, L. M. Brown, and S. D. Berger, 1989, "Nanofabrication using inorganic resists," Microelectron. Eng. 9, 557-560.

Mamola, K. C., R. J. Warmack, and T. L. Ferrell, 1987, "Surface-plasmon excitation by electrons in microlithographically produced channels," Phys. Rev. B 35, 2682-2686.

Manjavacas, A., and F. J. García de Abajo, 2009, "Robust plasmon waveguides in strongly interacting nanowire arrays," Nano Lett. 9, 1285-1289.

Marinopoulos, A. G., L. Reining, A. Rubio, and N. Vast, 2003, "Optical and loss spectra of carbon nanotubes: Depolarization effects and intertube interactions," Phys. Rev. Lett. 91, 046402.

Marks, L. D., 1982, "Observation of the image force for fast electrons near an $\mathrm{MgO}$ surface," Solid State Commun. 43, 727-729.

Marton, L., J. A. Simpson, H. A. Fowler, and N. Swanson, 1962, "Plural scattering of $20-\mathrm{keV}$ electrons in aluminum," Phys. Rev. 126, 182-192.

Masuda, H., and K. Fukuda, 1995, "Ordered metal nanohole arrays made by a two-step replication of honeycomb structures of anodic alumina," Science 268, 1466-1468.

Matloob, R., and A. Ghaffari, 2004, "Cerenkov radiation in a causal permeable medium," Phys. Rev. A 70, 052116.

Maxwell, J. C., 1891, Treatise on Electricity and Magnetism (Dover, New York).

Maxwell-Garnett, J. C., 1904, "Colours in metal glasses and in metallic films," Philos. Trans. R. Soc. London, Ser. A 203, 385-420.

Maxwell-Garnett, J. C., 1906, "Colours in metal glasses, in metallic films, and in metallic solutions, II," Philos. Trans. R. Soc. London, Ser. A 205, 237-288.

McComb, D. W., and A. Howie, 1995, "Valence loss spectra from $\mathrm{SiO}_{2}$ polymorphs of different density," Nucl. Instrum. Methods Phys. Res. B 96, 569-574.

McKenzie, D. R., and R. C. McPhedran, 1977, "Exact modeling of cubic lattice permittivity and conductivity," Nature (London) 265, 128-129.

McKenzie, D. R., R. C. McPhedran, and G. H. Derrick, 1978, "The conductivity of lattices of spheres. II. The body centred and face centred cubic lattices," Proc. R. Soc. London, Ser. A 362, 211-232.

Meiners, J. C., and S. R. Quake, 2000, "Femtonewton force spectroscopy of single extended DNA molecules," Phys. Rev.
Lett. 84, 5014-4017.

Mendoza, C. I., R. G. Barrera, and R. Fuchs, 1998, "Energy loss of electrons traveling parallel to the interface of a semiinfinite granular composite," Phys. Rev. B 57, 11193-11203.

Mendoza, C. I., R. G. Barrera, and R. Fuchs, 1999, "Electron energy loss in ordered arrays of polarizable spheres," Phys. Rev. B 60, 13831-13845.

Merano, M., S. Sonderegger, A. Crottini, S. Collin, P. Renucci, E. Pelucchi, A. Malko, M. H. Baier, E. Kapon, B. Deveaud, and J. D. Ganière, 2005, "Probing carrier dynamics in nanostructures by picosecond cathodoluminescence," Nature (London) 438, 479-482.

Mermin, N. D., 1970, "Lindhard dielectric function in the relaxation-time approximation," Phys. Rev. B 1, 2362-2363.

Mie, G., 1908, "Beiträge zur Optik trüber Medien, speziell kolloidaler Metallösungen," Ann. Phys. 25, 377-445.

Minoda, H., and N. Yamamoto, 2006, "Study on the origin of the anisotropic dielectric properties of the Au-adsorbed Si(001) vicinal surface," Surf. Interface Anal. 38, 1666-1669.

Mizuno, K., S. Ono, and O. Shimoe, 1975, "Interaction between coherent light waves and free electrons with a reflecting grating," Nature (London) 253, 184-185.

Mizuno, K., J. Pae, T. Nozokido, and K. Furuya, 1987, "Experimental evidence of the inverse Smith-Purcell effect," Nature (London) 328, 45-47.

Mkhoyan, K. A., T. Babinec, S. E. Maccagnano, E. J. Kirkland, and J. Silcox, 2007, "Separation of bulk and surface-losses in low-loss EELS measurements in STEM," Ultramicroscopy 107, 345-355.

Mock, J. J., M. Barbic, D. R. Smith, D. A. Schultz, and S. Schultz, 2002, "Shape effects in plasmon resonance of individual colloidal silver nanoparticles," J. Chem. Phys. 116, 6755-6759.

Monthioux, M., and V. L. Kuznetsov, 2006, "Who should be given the credit for the discovery of carbon nanotubes?," Carbon 44, 1621-1623.

Moran, M. J., 1992, "X-ray generation by the Smith-Purcell effect," Phys. Rev. Lett. 69, 2523-2526.

More, R. M., 1966, "Resonance in scattering of light by a Cherenkov electron," Phys. Rev. Lett. 16, 781-782.

Moreau, P., N. Brun, C. A. Walsh, C. Colliex, and A. Howie, 1997, "Relativistic effects in electron-energy-lossspectroscopy observations of the $\mathrm{Si} / \mathrm{SiO}_{2}$ interface plasmon peak," Phys. Rev. B 56, 6774-6781.

Müllejans, H., A. L. Beloch, A. Howie, and M. Tomita, 1993, "Secondary electron coincidence detection and time-of-flight spectroscopy," Ultramicroscopy 52, 360-368.

Müllejans, H., and A. L. Bleloch, 1992, "Ratio between the energy-loss spectrum in coincidence with secondary electrons and the normal energy-loss spectrum for thin carbon films in the carbon K-edge region,” Phys. Rev. B 46, 8597-8599.

Muller, D. A., L. Fitting Kourkoutis, M. Murfitt, J. H. Song, H. Y. Hwang, J. Silcox, N. Dellby, and O. L. Krivanek, 2008, "Atomic-scale chemical imaging of composition and bonding by aberration-corrected microscopy," Science 319, 1073-1076. Muller, D. A., and J. Silcox, 1995, "Delocalization in inelastic scattering," Ultramicroscopy 59, 195-213.

Muller, D. A., Y. Tzou, R. Raj, and J. Silcox, 1993, "Mapping sp2 and sp3 states of carbon at sub-nanometre spatial resolution," Nature (London) 366, 725-727.

Myroshnychenko, V., E. Carbó-Argibay, I. Pastoriza-Santos, J. Pérez-Juste, L. M. Liz-Marzán, and F. J. García de Abajo, 2008, "Modelling the optical response of highly faceted metal 
nanoparticles with a fully 3D boundary element method," Adv. Mater. 20, 4288-4293.

Myroshnychenko, V., J. Rodríguez-Fernández, I. PastorizaSantos, A. M. Funston, C. Novo, P. Mulvaney, L. M. LizMarzán, and F. J. García de Abajo, 2008, "Modelling the optical response of gold nanoparticles," Chem. Soc. Rev. 37, 1792-1805.

Nagao, T., S. Yaginuma, T. Inaoka, and T. Sakurai, 2006, "Onedimensional plasmon in an atomic-scale metal wire," Phys. Rev. Lett. 97, 116802.

Naumenko, G. A., V. A. Cha, B. N. Kalinin, Y. A. Popov, A. P. Potylitsyn, G. A. Saruev, and L. G. Sukhikh, 2008, "Focusing of transition radiation from a paraboloidal target," Nucl. Instrum. Methods Phys. Res. B 266, 3733-3737.

Nelayah, J., L. Gu, W. Sigle, C. T. Koch, I. Pastoriza-Santos, L. M. Liz-Marzán, and P. A. van Aken, 2009, "Direct imaging of surface-plasmon resonances on single triangular silver nanoprisms at optical wavelength using low-loss EFTEM imaging," Opt. Lett. 34, 1003-1005.

Nelayah, J., M. Kociak, O. Stéphan, F. J. García de Abajo, M. Tencé, L. Henrard, D. Taverna, I. Pastoriza-Santos, L. M. Liz-Marzán, and C. Colliex, 2007, "Mapping surface plasmons on a single metallic nanoparticle," Nat. Phys. 3, 348-353

Nelayah, J., O. Stéphan, M. Kociak, F. J. García de Abajo, L. Henrard, I. Pastoriza-Santos, L. M. Liz-Marzán, and C. Colliex, 2007, "Mapping surface plasmons on single metallic nanoparticles using sub-nm resolved EELS spectrumimaging," Microsc. Microanal. 13, 144-145.

Nellist, P. D., M. F. Chisholm, N. Dellby, O. L. Krivanek, M. F. Murfitt, Z. S. Szilagyi, A. R. Lupini, A. Borisevich, W. H. Sides, Jr., and S. J. Pennycook, 2004, "Direct sub-Angstrom imaging of a crystal lattice," Science 305, 1741.

N'Gom, M., J. Ringnalda, J. F. Mansfield, A. Agarwal, N. Kotov, N. J. Zaluzec, and T. B. Norris, 2008, "Single particle plasmon spectroscopy of silver nanowires and gold nanorods," Nano Lett. 8, 3200-3204.

Noguez, C., 2007, "Surface plasmons on metal nanoparticles: The influence of shape and physical environment," J. Phys. Chem. 111, 3806-3819.

Nojeh, A., B. Shan, K. Cho, and R. F. W. Pease, 2006, “ $A b$ initio modeling of the interaction of electron beams and single-walled carbon nanotubes," Phys. Rev. Lett. 96, 056802.

Novotny, L., R. X. Bian, and X. S. Xie, 1997, "Theory of nanometric optical tweezers," Phys. Rev. Lett. 79, 645-648.

Ochiai, T., and K. Ohtaka, 2004a, "Relativistic electron energy loss and induced radiation emission in two-dimensional metallic photonic crystals. I. Formalism and surface plasmon polariton," Phys. Rev. B 69, 125106.

Ochiai, T., and K. Ohtaka, 2004b, "Relativistic electron energy loss and induced radiation emission in two-dimensional metallic photonic crystals. II. Photonic band effects," Phys. Rev. B 69, 125107.

Ochiai, T., and K. Ohtaka, 2005, "Electron energy loss and Smith-Purcell radiation in two- and three-dimensional photonic crystals," Opt. Express 13, 7683-7698.

Ochiai, T., and K. Ohtaka, 2006, "Theory of unconventional Smith-Purcell radiation in finite-size photonic crystals," Opt. Express 14, 7378-7397.

Ohkuma, J., S. Okuda, and K. Tsumori, 1991, "Measurement of coherent Cherenkov radiation from an intense beam of a picosecond electron bunch,” Phys. Rev. Lett. 66, 1967-1969.

Ohtaka, K., and S. Yamaguti, 2001, "Theoretical study of the Smith-Purcell effect involving photonic cystals," Opt. Spec- trosc. 91, 506-512.

Oleshko, V. P., 2008, "Size confinement effects on electronic and optical properties of silver halide nanocrystals as probed by cryo-EFTEM and EELS," Int. J. Exergy 3, 41-46.

Olsen, H. A., and H. Kolbenstvedt, 1980, "Cherenkov radiation and transition radiation from small systems: Cherenkov radiation generated in a cylinder," Phys. Rev. A 21, 19871990.

Onishchenko, I. N., D. Yu. Sidorenko, and G. V. Sotnikov, 2002, "Structure of electromagnetic field excited by an electron bunch in a semi-infinite dielectric-filled waveguide," Phys. Rev. E 65, 066501.

Ortega, J. E., F. J. García de Abajo, P. M. Echenique, I. Manke, T. Kalka, M. Dähne, D. Ochs, S. L. Molodtsov, and A. Rubio, 1998, "Interface and bulk effects in the attenuation of low-energy electrons through $\mathrm{CaF}_{2}$ thin films," Phys. Rev. B 58, 2233-2239.

Osterwalder, J., T. Greber, S. Hüfner, and L. Schlapbach, 1990, "Photoelectron diffraction from core levels and plasmon-loss peaks of aluminum," Phys. Rev. B 41, 12495-12501.

Otto, A., 1967, "Theory of plasmon excitation in thin films by electrons of non-normal incidence," Phys. Status Solidi 22, 401-406.

Ouyang, F., P. E. Batson, and M. Isaacson, 1992, "Quantum size effects in the surface-plasmon excitation of small metallic particles by electron-energy-loss spectroscopy," Phys. Rev. B 46, 15421-15425.

Ouyang, F., and M. Isaacson, 1989a, "Accurate modeling of particle-substrate coupling of surface plasmon excitation in EELS," Ultramicroscopy 31, 345-349.

Ouyang, F., and M. Isaacson, 1989b, "Surface plasmon excitation of objects with arbitrary shape and dielectric constant," Philos. Mag. B 60, 481-492.

Oxley, M. P., and L. J. Allen, 1998, "Delocalization of the effective interaction for inner-shell ionization in crystals," Phys. Rev. B 57, 3273-3282.

Oxley, M. P., and S. J. Pennycook, 2008, "Image simulation for electron energy loss spectroscopy," Micron 39, 676-684.

Ozawa, L., 1990, Cathodoluminescence: Theory and Applications (VCH, New York).

Ozbay, E., 2006, "Plasmonics: Merging photonics and electronics at nanoscale dimensions," Science 311, 189-193.

Palik, E. D., 1985, Handbook of Optical Constants of Solids (Academic, San Diego).

Palik, E. D., 1991, Handbook of Optical Constants of Solids II (Academic, San Diego).

Palmer, R. E., and P. J. Rous, 1992, "Resonances in electron scattering by molecules on surfaces," Rev. Mod. Phys. 64, 383-440.

Patro, D. N., 1982, "Microscopic theory of synchrotronCherenkov radiation,” Phys. Rev. Lett. 49, 1083-1086.

Pawlak, D. A., K. Kolodziejak, K. Rozniatowski, R. Diduszko, M. Kaczkan, M. Malinowski, M. Piersa, J. Kisielewski, and T. Lukasiewicz, 2008, "PrAlO $\mathrm{Al}_{3}-\mathrm{PrAl}_{11} \mathrm{O}_{18}$ eutectic: Its microstructure and spectroscopic properties," Cryst. Growth Des. 8, 1243-1249.

Peale, R. E., O. Lopatiuk, J. Cleary, S. Santos, J. Henderson, D. Clark, L. Chernyak, T. A. Winningham, E. Del Barco, H. Heinrich, and W. R. Buchwald, 2008, "Propagation of highfrequency surface plasmons on gold," J. Opt. Soc. Am. B 25, 1708-1713.

Pendry, J. B., and L. Martín-Moreno, 1994, "Energy loss by 
charged particles in complex media," Phys. Rev. B 50, 50625073.

Peng, J. L., R. P. Fehlhaber, L. A. Bursill, and D. G. McCulloch, 2001, "Analysis of nanocrystalline diamond powder by scanning transmission electron microscopy," J. Appl. Phys. 89, 6204-6213.

Pennycook, S. J., 2008, "Investigating the optical properties of dislocations by scanning transmission electron microscopy," Scanning 30, 287-298.

Persson, B. N. J., and A. Baratoff, 1992, "Theory of photon emission in electron tunneling to metallic particles," Phys. Rev. Lett. 68, 3224-3227.

Pettit, R. B., J. Silcox, and R. Vincent, 1975, "Measurement of surface-plasmon dispersion in oxidized aluminum films," Phys. Rev. B 11, 3116-3123.

Pflüger, J., and J. Fink, 1991, in Handbook of Optical Constants in Solids II, edited by E. D. Palik (Academic, San Diego), Chap. 13, pp. 293-311.

Pflüger, J., J. Fink, W. Weber, K. P. Bohnen, and G. Crecelius, 1984, "Dielectric properties of $\mathrm{TiC}_{x}, \mathrm{TiN}_{x}, \mathrm{VC}_{x}$, and $\mathrm{VN}_{x}$ from 1.5 to $40 \mathrm{eV}$ determined by electron-energy-loss spectroscopy," Phys. Rev. B 30, 1155-1163.

Pflüger, J., J. Fink, W. Weber, K. P. Bohnen, and G. Crecelius, 1985, "Dielectric properties of $\mathrm{ZrN}, \mathrm{NbC}$, and $\mathrm{NbN}$ as determined by electron-energy-loss spectroscopy," Phys. Rev. B 31, 1244-1247.

Pijper, F. J., and P. Kruit, 1991, "Detection of energy-selected secondary electrons in coincidence with energy-loss events in thin carbon foils," Phys. Rev. B 44, 9192-9200.

Pines, D., and D. Bohm, 1952, "A collective description of electron interactions: II. Collective vs individual particle aspects of the interactions," Phys. Rev. 85, 338-353.

Pines, D., and P. Nozières, 1966, The Theory of Quantum Liquids (Benjamin, New York).

Pitarke, J. M., V. M. Silkin, E. V. Chulkov, and P. M. Echenique, 2007, "Theory of surface plasmons and surfaceplasmon polaritons," Rep. Prog. Phys. 70, 1-87.

Pochon, S., K. F. MacDonald, R. J. Knize, and N. I. Zheludev, 2004, "Phase coexistence in gallium nanoparticles controlled by electron excitation," Phys. Rev. Lett. 92, 145702.

Pogorzelski, R., and C. Yeh, 1973, "Difraction radiation from a charged particle moving through a penetrable sphere," Phys. Rev. A 8, 137-144.

Pokrant, S., R. Pantel, and M. Cheynet, 2006, "Physical characterization by valence electron energy loss spectroscopy," Microelectron. Eng. 83, 2364-2367.

Potapov, P. L., J. Verbeeck, P. Schattschneider, H. Lichte, and D. van Dyck, 2007, "Inelastic electron holography as a variant of the Feynman thought experiment," Ultramicroscopy 107, 559-567.

Potylitsyn, A. P., 1998, "Transition radiation and diffraction radiation. Similarities and differences," Nucl. Instrum. Methods Phys. Res. B 145, 169-179.

Powell, C. J., 1965, "Differences in the characteristic electron energy-loss spectra of solid and liquid bismuth," Phys. Rev. Lett. 15, 852-854.

Powell, C. J., 1968, "Characteristic energy losses of 8-keV electrons in liquid Al, Bi, In, Ga, Hg, and Au," Phys. Rev. 175, 972-982.

Powell, C. J., and J. B. Swan, 1959, "Origin of the characteristic electron energy losses in aluminum," Phys. Rev. 115, 869875 .

Pratesi, G., A. L. Guidice, S. Vishnevsky, C. Manfredotti, and
C. Cipriani, 2003, "Cathodoluminescence investigations on the Popigai, Ries, and Lappajärvi impact diamonds," Am. Mineral. 88, 1778-1787.

Purcell, E. M., 1946, "Spontaneous emission probabilities at radio frequencies," Phys. Rev. 69, 681.

Purcell, E. M., and C. R. Pennypacker, 1973, "Scattering and absorption of light by nonspherical dielectric grains," Astrophys. J. 186, 705-714.

Raether, H., 1967, "Surface plasma oscillations as a tool for surface examinations," Surf. Sci. 8, 233-246.

Raether, H., 1980, Excitation of Plasmons and Interband Transitions by Electrons, Springer Tracks in Modern Physics Vol. 88 (Springer-Verlag, Berlin).

Raether, H., 1988, Surface Plasmons on Smooth and Rough Surfaces and on Gratings, Springer Tracks in Modern Physics Vol. 111 (Springer-Verlag, Berlin).

Rafferty, B., and L. M. Brown, 1998, "Direct and indirect transitions in the region of the band gap using electron-energyloss spectroscopy," Phys. Rev. B 58, 10326-10337.

Rang, M., A. C. Jones, F. Zhou, Z. Y. Li, B. J. Wiley, Y. Xia, and M. B. Raschke, 2008, "Optical near-field mapping of plasmonic nanoprisms," Nano Lett. 8, 3357-3363.

Rapoport, W. R., and C. P. Khattak, 1988, "Titanium sapphire laser characteristics,” Appl. Opt. 27, 2677-2684.

Reed, B. W., J. M. Chen, N. C. MacDonald, J. Silcox, and G. F. Bertsch, 1999, "Fabrication and STEM/EELS measurements of nanometer-scale silicon tips and filaments," Phys. Rev. B 60, 5641-5652.

Righini, M., G. Volpe, C. Girard, D. Petrov, and R. Quidant, 2008, "Surface plasmon optical tweezers: Tunable optical manipulation in the femtonewton range," Phys. Rev. Lett. 100, 186804.

Ritchie, R. H., 1957, "Plasma losses by fast electrons in thin films," Phys. Rev. 106, 874-881.

Ritchie, R. H., E. T. Arakawa, J. J. Cowan, and R. N. Hamm, 1968, "Surface-plasmon resonance effect in grating diffraction," Phys. Rev. Lett. 21, 1530-1533.

Ritchie, R. H., J. C. Ashley, and L. C. Emerson, 1964, "Optical bremsstrahlung and transition radiation from irradiated media," Phys. Rev. 135, A759-A763.

Ritchie, R. H., and H. B. Eldridge, 1962, "Optical emission from irradiated foils. I,” Phys. Rev. 126, 1935-1947.

Ritchie, R. H., and A. Howie, 1988, "Inelastic-scattering probabilities in scanning-transmission electron-microscopy," Philos. Mag. A 58, 753-767.

Ritchie, R. H., and A. L. Marusak, 1966, "The surface plasmon dispersion relation for an electron gas," Surf. Sci. 4, 234-240. Rivacoba, A., N. Zabala, and J. Aizpurua, 2000, "Image potential in scanning transmission electron microscopy," Prog. Surf. Sci. 65, 1-64.

Rivacoba, A., N. Zabala, and P. M. Echenique, 1992, "Theory of energy loss in scanning transmission electron microscopy of supported small particles," Phys. Rev. Lett. 69, 3362-3365. Rocca, M., 1995, "Low-energy EELS investigation of surface electronic excitations on metals," Surf. Sci. Rep. 22, 1-71.

Rodríguez-Lorenzo, L., R. A. Álverez-Puebla, I. PastorizaSantos, S. Mazzucco, O. Stéphan, M. Kociak, L. M. LizMarzán, and F. J. García de Abajo, 2009, "Zeptomol detection through controlled ultrasensitive surface-enhanced Raman scattering," J. Am. Chem. Soc. 131, 4616-4618.

Rodt, S., A. Schliwa, K. Pötschke, F. Guffarth, and D. Bimberg, 2005, "Correlation of structural and few-particle properties of self-organized InAs/GaAs quantum dots," Phys. 
Rev. B 71, 155325.

Rogacheva, A. V., V. A. Fedotov, A. S. Schwanecke, and N. I. Zheludev, 2006, "Giant gyrotropy due to electromagneticfield coupling in a bilayered chiral structure," Phys. Rev. Lett. 97, 177401.

Rojas, R., F. Claro, and R. Fuchs, 1988, "Nonlocal response of a small coated sphere," Phys. Rev. B 37, 6799-6807.

Romero, I., J. Aizpurua, G. W. Bryant, and F. J. García de Abajo, 2006, "Plasmons in nearly touching metallic nanoparticles: Singular response in the limit of touching dimers," Opt. Express 14, 9988-9999.

Rösler, M., and W. Brauer, 1991, Particle Induced Electron Emission I, Springer Tracts in Modern Physics Vol. 122 (Springer-Verlag, Berlin), pp. 1-65.

Ruppin, R., 1978, "Plasmon frequencies of small metal spheres," J. Phys. Chem. Solids 39, 233-237.

Ruppin, R., 1982, "Surface modes of two spheres," Phys. Rev. B 26, 3440-3444.

Ruska, E., 1987, "The development of the electron microscope and of electron microscopy," Rev. Mod. Phys. 59, 627-638.

Ruthermann, G., 1948, "Diskrete energieverluste mittelschneller elektronen beim durchgang durch dunne folien," Ann. Phys. 2, 113-134.

Ružička, J., and V. P. Zrelov, 1993, "Optical transition radiation in a transparent medium and its relation to the VavilovCherenkov radiation," Czech. J. Phys. 43, 551-567.

Saito, Y., H. Shinohara, and A. Ohshita, 1991, "Bulk plasmons in solid C 60 ," Jpn. J. Appl. Phys., Part 2 30, L1068-L1070.

Salisbury, W. W., 1970, "Generation of light from free electrons,” J. Opt. Soc. Am. 60, 1279-1284.

Sánchez-Iglesias, A., I. Pastoriza-Santos, J. Pérez-Juste, B. Rodríguez-González, F. J. García de Abajo, and L. M. LizMarzán, 2006, "Synthesis and optical properties of gold nanodecahedra with size control," Adv. Mater. 18, 2529-2534.

Sander, M. S., R. Gronsky, Y. M. Lin, and M. S. Dresselhaus, 2001, "Plasmon excitation modes in nanowire arrays," J. Appl. Phys. 89, 2733-2736.

Sarid, D., 1981, "Long-range surface-plasma waves on very thin metal films," Phys. Rev. Lett. 47, 1927-1930.

Sauer, R., H. Sternschulte, S. Wahl, K. Thonke, and T. R. Anthony, 2000, "Revised fine splitting of excitons in diamond," Phys. Rev. Lett. 84, 4172-4175.

Schaffer, B., U. Hohenester, A. Trügler, and F. Hofer, 2009, "High-resolution surface plasmon imaging of gold nanoparticles by energy-filtered transmission electron microscopy," Phys. Rev. B 79, 041401(R).

Schattschneider, P., F. Födermayr, and D. S. Su, 1987, "Coherent double-plasmon excitation in aluminum," Phys. Rev. Lett. 59, 724-727.

Schattschneider, P., S. Rubino, C. Hébert, J. Rusz, J. Kuneš, P. Novák, E. Carlino, M. Fabrizioli, G. Panaccione, and G. Rossi, 2006, "Detection of magnetic circular dichroism using a transmission electron microscope," Nature (London) 441, 486-488.

Schattschneider, P., and W. S. M. Werner, 2005, "Coherence in electron energy loss spectrometry," J. Electron Spectrosc. Relat. Phenom. 143, 81-95.

Scheinfein, M., A. Muray, and M. Isaacson, 1985, "Electron energy loss spectroscopy across a metal-insulator interface at sub-nanometer spatial resolution," Ultramicroscopy 16, 233239.

Scheinfein, M. R., J. Drucker, and J. K. Weiss, 1993, "Secondary-electron production pathways determined by co- incidence electron spectroscopy," Phys. Rev. B 47, 4068-4071. Schieber, D., and L. Schächter, 1998, "Reaction forces on a relativistic point charge moving above a dielectric or a metallic half-space," Phys. Rev. E 57, 6008-6015.

Schieber, J., D. Krinsley, and L. Riciputi, 2000, "Diagenetic origin of quartz silt in mudstones and implications for silica cycling," Nature (London) 406, 981-985.

Schilling, J., and H. Raether, 1973, "Energy gain of fast electrons interacting with surface plasmons," J. Phys. Condens. Matter 6, L358-L360.

Schmeits, M., 1989, "Surface-plasmon coupling in cylindrical pores,” Phys. Rev. B 39, 7567-7577.

Schmeits, M., and L. Dambly, 1991, "Fast-electron scattering by bispherical surface-plasmon modes," Phys. Rev. B 44, 12706-12712.

Schuster, S. C., R. V. Swanson, L. A. Alex, R. B. Bourret, and M. I. Simon, 1993, "Assembly and function of a quaternary signal-transduction complex monitored by surface-plasmon resonance," Nature (London) 365, 343-347.

Shibata, Y., S. Hasebe, K. Ishi, S. Ono, M. Ikezawa, T. Nakazato, M. Oyamada, S. Urasawa, T. Takahashi, T. Matsuyama, K. Kobayashi, and Y. Fujita, 1998, "Coherent Smith-Purcell radiation in the millimeter-wave region from a short-bunch beam of relativistic electrons," Phys. Rev. E 57, 1061-1074.

Shieh, S. Y., and R. H. Ritchie, 1970, "Simultaneous generation of transition radiation and bremsstrahlung from a thin foil. I," Phys. Rev. B 2, 1646-1651.

Shiles, E., T. Sasaki, M. Inokuti, and D. Y. Smith, 1980, "Selfconsistency and sum-rule tests in the Kramers-Kronig analysis of optical data: Applications to aluminum," Phys. Rev. B 22, 1612-1628.

Shubina, T. V., et al., 2004, "Mie resonances, infrared emission, and the band gap of InN," Phys. Rev. Lett. 92, 117407.

Sigle, W., J. Nelayah, C. T. Koch, and P. A. van Aken, 2009, "Electron energy losses in Ag nanoholes from localized surface plasmon resonances to rings of fire," Opt. Lett. 34, 21502152.

Simonsen, A. C., F. Yubero, and S. Tougaard, 1997, "Quantitative model of electron energy loss in XPS," Phys. Rev. B 56, 1612-1619.

Skryabin, D. V., F. Luan, J. C. Knight, and P. S. J. Russell, 2003, "Soliton self-frequency shift cancellation in photonic crystal fibers," Science 301, 1705-1708.

Smith, N. V., 1985, "Phase analysis of image states and surface states associated with nearly-free-electron band gaps," Phys. Rev. B 32, 3549-3555.

Smith, S. J., and E. M. Purcell, 1953, "Visible light from localized surface charges moving across a grating," Phys. Rev. 92, 1069.

Sonderegger, S., E. Feltin, M. Merano, A. Crottini, J. F. Carlin, R. Sachot, B. Deveaud, N. Grandjean, and J. D. Ganière, 2006, "High spatial resolution picosecond cathodoluminescence of InGaN quantum wells," Appl. Phys. Lett. 89, 232109.

Sosa, I. O., C. I. Mendoza, and R. G. Barrera, 2001, "Calculation of electron-energy-loss spectra of composites and selfsimilar structures," Phys. Rev. B 63, 144201.

Stefanou, N., V. Yannopapas, and A. Modinos, 2000, "MULTEM 2: A new version of the program for transmission and band-structure calculations of photonic crystals," Comput. Phys. Commun. 132, 189-196.

Steinmann, W., 1960, "Experimental verification of radiation of plasma oscillations in thin silver films," Phys. Rev. Lett. 5, 
470-472.

Stéphan, O., D. Taverna, M. Kociak, K. Suenaga, L. Henrard, and C. Colliex, 2002, "Dielectric response of isolated carbon nanotubes investigated by spatially resolved electron energyloss spectroscopy: From multiwalled to single-walled nanotubes," Phys. Rev. B 66, 155422.

Stern, E. A., and R. A. Ferrell, 1960, "Surface plasma oscillations of a degenerate electron gas," Phys. Rev. 120, 130-136.

Stevens, T. E., J. K. Wahlstrand, J. Kuhl, and R. Merlin, 2001, "Cherenkov radiation at speeds below the light threshold: Phonon-assisted phase matching," Science 291, 627-630.

Stöckli, T., J. M. Bonard, P. A. Stadelmann, and A. Châtelain, 1997, "EELS investigation of plasmon excitations in aluminum nanospheres and carbon nanotubes," Z. Phys. D: At., Mol. Clusters 40, 425-428.

Stöger-Pollach, M., 2008, "Optical properties and bandgaps from low loss EELS: Pitfalls and solutions," Micron 39, 10921110.

Stöger-Pollach, M., H. Franco, P. Schattschneider, S. Lazar, B. Schaffer, W. Grogger, and H. W. Zandbergen, 2006, "Cherenkov losses: A limit for bandgap determination and KramersKronig analysis," Micron 37, 396-402.

Stöger-Pollach, M., A. Laister, and P. Schattschneider, 2008, "Treating retardation effects in valence EELS spectra for Kramers-Kronig analysis," Ultramicroscopy 108, 439-444.

Stöger-Pollach, M., and P. Schattschneider, 2007, "The influence of relativistic energy losses on bandgap determination using valence EELS," Ultramicroscopy 107, 1178-1185.

Stolojan, V., P. Moreau, M. J. Goringe, and S. R. P. Silva, 2006, "Subnanometer-resolved measurement of the tunneling effective mass using bulk plasmons," Appl. Phys. Lett. 88, 122109.

Su, D. S., H. W. Zandbergen, P. C. Tiemeijer, G. Kothleitner, M. Hävecker, C. Hébert, A. Knop-Gericke, B. H. Freitag, F. Hofer, and R. Schlögl, 2003, "High resolution EELS using monochromator and high performance spectrometer: Comparison of $\mathrm{V}_{2} \mathrm{O}_{5}$ ELNES with NEXAFS and band structure calculations," Micron 34, 235-238.

Sun, S., S. Shi, and R. Leapman, 1993, "Water distributions of hydrated biological specimens by valence electron energy loss spectroscopy," Ultramicroscopy 50, 127-139.

Talley, C. E., J. B. Jackson, C. Oubre, N. K. Grady, C. W. Hollars, S. M. Lane, T. R. Huser, P. Nordlander, and N. J. Halas, 2005, "Surface-enhanced Raman scattering from individual Au nanoparticles and nanoparticle dimer substrates," Nano Lett. 5, 1569-1574.

Tamm, I., 1939, "Radiation emitted by uniformly moving electrons," J. Phys. (Moscow) 1, 439-454.

Tanuma, S., C. J. Powell, and D. R. Penn, 1994, "Calculations of electron inelastic mean free paths. V. Data for 14 organic compounds over the 50-2000 eV range," Surf. Interface Anal. 21, 165-176.

Taverna, D., M. Kociak, V. Charbois, and L. Henrard, 2002, "Electron energy-loss spectrum of an electron passing near a locally anisotropic nanotube," Phys. Rev. B 66, 235419.

Temkin, R., 1998, "Scanning with ease through the far infrared," Science 280, 854.

Teng, Y. Y., and E. A. Stern, 1967, "Plasma radiation from metal grating surfaces," Phys. Rev. Lett. 19, 511-514.

Ter-Mikaelian, M. L., 1972, High-Energy Electromagnetic Processes in Condensed Media (Wiley, New York).

Terauchi, M., M. Tanaka, K. Tsuno, and M. Ishida, 1999, "Development of a high energy resolution electron energy-loss spectroscopy microscope," J. Microsc. 194, 203-209.

Tomaš, M. S., A. A. Lucas, and M. Šunjić, 1972, "Emission of radiation by charged particles reflected from solid surfaces," Solid State Commun. 10, 1181-1184.

Tomaš, M. S., A. A. Lucas, M. Šunjić, and D. Juretić, 1974, "Coherent surface bremsstrahlung in low-energy-electron diffraction and reflection-high-energy-electron diffraction," Phys. Rev. B 9, 1489-1498.

Tomita, S., S. Yoda, R. Uchiyama, S. Ishii, K. Sasa, T. Kaneko, and H. Kudo, 2006, "Nonadditivity of convoy- and secondary-electron yields in the forward-electron emission from thin carbon foils under irradiation of fast carbon-cluster ions," Phys. Rev. A 73, 060901(R).

Toraldo di Francia, G., 1960, "On the theory of some Cherenkovian effects," Nuovo Cimento 16, 61-77.

Trügler, A., and U. Hohenester, 2008, "Strong coupling between a metallic nanoparticle and a single molecule," Phys. Rev. B 77, 115403.

Tu, Y. H., C. M. Kwei, and C. J. Tung, 2006, "Inelastic interactions of electrons with cylindrical interfaces," Surf. Sci. 600, 820-824.

Ugarte, D., C. Colliex, and P. Trebbia, 1992, "Surface- and interface-plasmon modes on small semiconducting spheres," Phys. Rev. B 45, 4332-4343.

Uhlemann, S., and M. Haider, 1998, "Residual wave aberrations in the first spherical aberration corrected transmission electron microscope," Ultramicroscopy 72, 109-119.

Ulrich, R., and M. Tacke, 1973, "Submillimeter waveguiding on periodic metal structure," Appl. Phys. Lett. 22, 251-253.

Urata, J., M. Goldstein, M. F. Kimmitt, A. Naumov, C. Platt, and J. E. Walsh, 1998, "Superradiant Smith-Purcell emission," Phys. Rev. Lett. 80, 516-519.

van Attekum, P. M. T. M., and J. M. Trooster, 1978, "Bulk- and surface-plasmon-loss intensities in photoelectron, Auger, and electron-energy-loss spectra of Al metal," Phys. Rev. B 18, 3872-3883.

van Benthem, K., C. Elsässer, and R. H. French, 2001, "Bulk electronic structure of $\mathrm{SrTiO}_{3}$ : Experiment and theory," J. Appl. Phys. 90, 6156-6164.

van de Hulst, H. C., 1981, Light Scattering by Small Particles (Dover, New York).

van den Berg, P. M., 1973, "Smith-Purcell radiation from a point charge moving parallel to a reflection grating," J. Opt. Soc. Am. 63, 1588-1597.

van den Berg, P. M., and T. H. Tan, 1974, "Smith-Purcell radiation from a line charge moving parallel to a reflection grating with rectangular profile," J. Opt. Soc. Am. 64, 325-328.

Van Hove, L., 1953, "The occurrence of singularities in the elastic frequency distribution of a crystal," Phys. Rev. 89, 1189-1193.

van Wijngaarden, J. T., E. Verhagen, A. Polman, C. E. Ross, H. J. Lezec, and H. A. Atwater, 2006, "Direct imaging of propagation and damping of near-resonance surface plasmon polaritons using cathodoluminescence spectroscopy," Appl. Phys. Lett. 88, 221111.

Varela, M., S. D. Findlay, A. R. Lupini, H. M. Christen, A. Y. Borisevich, N. Dellby, O. L. Krivanek, P. D. Nellist, M. P. Oxley, L. J. Allen, and S. J. Pennycook, 2004, "Spectroscopic imaging of single atoms within a bulk solid," Phys. Rev. Lett. 92, 095502.

Varela, M., M. P. Oxley, K. G. Roberts, J. Garcia-Barriocanal, A. R. Lupini, S. N. Rashkeev, C. Leon, K. M. Krishnan, J. Santamaria, S. T. Pantelides, and S. J. Pennycook, 2007, 
"Spectroscopic imaging of oxide interfaces with aberration corrected probes," Microsc. Microanal. 13, 142-143.

Vast, N., L. Reining, V. Olevano, P. Schattschneider, and B. Jouffrey, 2002, "Local field effects in the electron energy loss spectra of rutile $\mathrm{TiO}_{2}$," Phys. Rev. Lett. 88, 037601.

Verbeeck, J., 2006, "Interpretation of 'Energy-filtered electron-diffracted beam holography' by R. A. Herring," Ultramicroscopy 106, 461-465.

Verbeeck, J., G. Bertoni, and P. Schattschneider, 2008, “The Fresnel effect of a defocused biprism on the fringes in inelastic holography," Ultramicroscopy 108, 263-269.

Veselago, V. G., 1968, "Electrodynamics of substances with simultaneously negative values of sigma and mu," Sov. Phys. Usp. 10, 509-514.

Vesseur, E. J. R., R. de Waele, M. Kuttge, and A. Polman, 2007, "Direct observation of plasmonic modes in Au nanowires using high-resolution cathodoluminescence spectroscopy," Nano Lett. 7, 2843-2846.

Vesseur, E. J. R., R. de Waele, H. J. Lezec, H. A. Atwater, F. J. García de Abajo, and A. Polman, 2008, "Surface plasmon polariton modes in a single-crystal $\mathrm{Au}$ nanoresonator fabricated using focused-ion-beam milling," Appl. Phys. Lett. 92, 083110.

Vesseur, E. J. R., F. J. García de Abajo, and A. Polman, 2009, "Modal decomposition of surface-plasmon whispering gallery resonators," Nano Lett. 9, 3147-3150.

Vincent, R., and J. Silcox, 1973, "Dispersion of radiative surface plasmons in aluminum films by electron scattering," Phys. Rev. Lett. 31, 1487-1490.

Von Festenberg, C., and E. Kröger, 1968, "Retardation effects for the electron energy loss probability in $\mathrm{GaP}$ and $\mathrm{Si}$," Phys. Lett. 26A, 339-340.

Wagner, D., 1966, "Oberflachenwellen im Elektronenplasma," Z. Naturforsch. A 21, 634.

Walls, M. G., and A. Howie, 1989, "Dielectric theory of localised valence energy loss spectroscopy," Ultramicroscopy 28, 40-42.

Walsh, C. A., 1991, “An analytical expression for the energyloss of fast electrons traveling parallel to the axis of a cylindrical interface," Philos. Mag. B 63, 1063-1078.

Wang, P., A. J. D'Alfonso, S. D. Findlay, L. J. Allen, and A. L. Bleloch, 2008, "Contrast reversal in atomic-resolution chemical mapping," Phys. Rev. Lett. 101, 236102.

Wang, X., X. G. Zhang, Q. Yu, and B. N. Harmon, 1993, "Multiple-scattering theory for electromagnetic waves," Phys. Rev. B 47, 4161-4167.

Wang, Z. L., 1996, "Valence electron excitations and plasmon oscillations in thin films, surfaces, interfaces and small particles," Micron 27, 265-299.

Wang, Z. L., H. L. W. Chan, H. L. Li, and J. H. Hao, 2008, "Highly efficient low-voltage cathodoluminescence of $\mathrm{LaF}_{3}: \mathrm{Ln}^{3+}\left(\mathrm{Ln}=\mathrm{Eu}^{3+}, \mathrm{Ce}^{3+}, \mathrm{Tb}^{3+}\right)$ spherical particles," Appl. Phys. Lett. 93, 141106.

Wang, Z. L., and J. M. Cowley, 1987a, "Excitation of the supported metal particle surface plasmon with external electron beam," Ultramicroscopy 21, 335-345.

Wang, Z. L., and J. M. Cowley, 1987b, "Generation of surface plasmon excitation of supported metal particles by an external electron beam," Ultramicroscopy 21, 347-365.

Wang, Z. L., and J. M. Cowley, 1987c, "Size and shape dependence of the surface plasmon frequencies for supported metal particle systems," Ultramicroscopy 23, 97-107.

Wang, Z. L., and J. M. Cowley, 1987d, "Surface plasmon exci- tation for supported metal particles," Ultramicroscopy 21, 77-93.

Wang, Z. L., and J. M. Cowley, 1988, "Reflection electron energy loss spectroscopy (REELS): A technique for the study of surfaces," Surf. Sci. 193, 501-512.

Ward, B. W., J. A. Notte, and N. P. Economou, 2006, "Helium ion microscope: A new tool for nanoscale microscopy and metrology," J. Vac. Sci. Technol. B 24, 2871-2874.

Warmack, R. J., R. S. Becker, V. E. Anderson, R. H. Ritchie, Y. T. Chu, J. Little, and T. L. Ferrell, 1984, "Surface-plasmon excitation during aloof scattering of low-energy electrons in micropores in a thin metal foil," Phys. Rev. B 29, 4375-4381. Watanabe, H., 1956, "Experimental evidence for the collective nature of the characteristic energy loss of electrons in solids-Studies on the dispersion relation of plasma frequency,” J. Phys. Soc. Jpn. 11, 112-119.

Went, M. R., M. Vos, and W. S. M. Werner, 2008, "Extracting the Ag surface and volume loss functions from reflection electron energy loss spectra," Surf. Sci. 602, 2069-2077.

Werner, W. S. M., 2006, "Dielectric function of Cu, Ag, and Au obtained from reflection electron energy loss spectra, optical measurements, and density functional theory," Appl. Phys. Lett. 89, 213106.

Werner, W. S. M., M. R. Went, and M. Vos, 2007, "Surface plasmon excitation at a Au surface by $150-40000 \mathrm{eV}$ electrons," Surf. Sci. 601, L109-L113.

Williams, P., C. Lévy-Clément, A. Albu-Yaron, N. Brun, and C. Colliex, 2000, "Near-field electron energy loss spectroscopy in porous silicon," J. Porous Mater. 7, 159-163.

Winter, H., 2002, "Collisions of atoms and ions with surfaces under grazing incidence," Phys. Rep. 367, 387-582.

Winther, A., and K. Alder, 1979, "Relativistic Coulomb excitation," Nucl. Phys. A 319, 518-532.

Woods, K. J., J. E. Walsh, R. E. Stoner, H. G. Kirk, and R. C. Fernow, 1995, "Forward directed Smith-Purcell radiation from relativisitic electrons," Phys. Rev. Lett. 74, 3808-3811.

Xi, S., H. Chen, T. Jiang, L. Ran, J. Huangfu, B. I. Wu, J. A. Kong, and M. Chen, 2009, "Experimental verification of reversed Cherenkov radiation in left-handed metamaterial," Phys. Rev. Lett. 103, 194801.

Xu, H., E. J. Bjerneld, M. Käll, and L. Börjesson, 1999, "Spectroscopy of single hemoglobin molecules by surface enhanced Raman scattering," Phys. Rev. Lett. 83, 4357-4360.

Xu, J., Y. Dong, and X. Zhang, 2008, "Electromagnetic interactions between a fast electron beam and metamaterial cloaks," Phys. Rev. E 78, 046601.

$\mathrm{Xu}$, J., and X. Zhang, 2008, "Relativistic energy loss and induced photon emission in the interaction of a left-handed sphere with an external electron beam," Phys. Lett. A 372, 1129-1134.

Yacobi, B. G., and D. B. Holt, 1986, "Cathodoluminescence scanning electron-microscopy of semiconductors," J. Appl. Phys. 59, R1-R24.

Yamaguti, S., J. Inoue, O. Haeberlé, and K. Ohtaka, 2002, "Photonic crystals versus diffraction gratings in Smith-Purcell radiation," Phys. Rev. B 66, 195202.

Yamamoto, K., et al., 2004, "Observation of millimeter-wave radiation generated by the interaction between an electron beam and a photonic crystal," Phys. Rev. E 69, 045601(R).

Yamamoto, N., K. Araya, and F. J. García de Abajo, 2001, "Photon emission from silver particles induced by a highenergy electron beam," Phys. Rev. B 64, 205419.

Yamamoto, N., K. Araya, A. Toda, and H. Sugiyama, 2001, 
"Light emission from surfaces, thin films and particles induced by high-energy electron beam," Surf. Interface Anal. 31, 79-86.

Yamamoto, N., S. Bhunia, and Y. Watanabe, 2006, "Polarized cathodoluminescence study of InP nanowires by transmission electron microscopy," Appl. Phys. Lett. 88, 153106.

Yamamoto, N., M. Nakano, and T. Suzuki, 2006, "Light emission by surface plasmons on nanostructures of metal surfaces induced by high-energy electron beams," Surf. Interface Anal. 38, 1725-1730.

Yamamoto, N., H. Sugiyama, and A. Toda, 1996, "Cherenkov and transition radiation from thin plate crystals detected in the transmission electron microscope," Proc. R. Soc. London, Ser. A 452, 2279-2301.

Yamamoto, N., and T. Suzuki, 2008, "Conversion of surface plasmon polaritons to light by a surface step," Appl. Phys. Lett. 93, 093114.

Yamamoto, N., A. Toda, and K. Araya, 1996, "Imaging of transition radiation from thin films on a silicon substrate using a light detection system combined with TEM," J. Electron Microsc. 45, 64-72.

Yurtsever, A., M. Couillard, and D. A. Muller, 2008, "Formation of guided Cherenkov radiation in silicon-based nanocomposites," Phys. Rev. Lett. 100, 217402.

Zabala, N., and P. M. Echenique, 1990, "Energy loss of fast electrons moving near plane boundaries with dispersive media," Ultramicroscopy 32, 327-335.
Zabala, N., A. Rivacoba, and P. M. Echenique, 1989, "Energy loss of electrons travelling through cylindrical holes," Surf. Sci. 209, 465-480.

Zabala, N., A. Rivacoba, and P. M. Echenique, 1997, "Coupling effects in the excitations by an electron beam near close particles," Phys. Rev. B 56, 7623-7635.

Zaremba, E., 1985, "Van der Waals interaction between an atom and a surface defect," Surf. Sci. 151, 91-102.

Zhang, L., R. Erni, J. Verbeeck, and G. Van Tendeloo, 2008, "Retrieving the dielectric function of diamond from valence electron energy-loss spectroscopy," Phys. Rev. B 77, 195119.

Zhao, Z., and B. Lü, 2008, "Acceleration of electrons by a Bessel-Gaussian beam in vacuum," Opt. Quantum Electron. 40, 615-622.

Zheludev, N. I., 2008, "What diffraction limit?," Nature Mater. 7, 420-422.

Zia, R., J. A. Schuller, A. Chandran, and M. L. Brongersma, 2006, "Plasmonics: The next chip-scale technology," Mater. Today 9, 20-27.

Ziegler, J. F., 1999, "Stopping of energetic light ions in elemental matter," J. Appl. Phys. 85, 1249-1272.

Zrelov, V. P., and J. Ružička, 1989, “Analysis of Tamm's problem on charge radiation at its uniform motion over a finite trajectory," Czech. J. Phys., Sect. B 39, 368-383.

Zuloaga, J., E. Prodan, and P. Nordlander, 2009, "Quantum description of the plasmon resonances of a nanoparticle dimer," Nano Lett. 9, 887-891. 